\title{
Dietary acrylamide intake and human cancer risk
}

Citation for published version (APA):

Hogervorst, J. G. F. (2009). Dietary acrylamide intake and human cancer risk. [Doctoral Thesis, Maastricht University]. Datawyse / Universitaire Pers Maastricht. https://doi.org/10.26481/dis.20091002jh

Document status and date:

Published: 01/01/2009

DOI:

10.26481/dis.20091002jh

Document Version:

Publisher's PDF, also known as Version of record

\section{Please check the document version of this publication:}

- A submitted manuscript is the version of the article upon submission and before peer-review. There can be important differences between the submitted version and the official published version of record.

People interested in the research are advised to contact the author for the final version of the publication, or visit the DOI to the publisher's website.

- The final author version and the galley proof are versions of the publication after peer review.

- The final published version features the final layout of the paper including the volume, issue and page numbers.

Link to publication

\footnotetext{
General rights rights.

- You may freely distribute the URL identifying the publication in the public portal. please follow below link for the End User Agreement:

www.umlib.nl/taverne-license

Take down policy

If you believe that this document breaches copyright please contact us at:

repository@maastrichtuniversity.nl

providing details and we will investigate your claim.
}

Copyright and moral rights for the publications made accessible in the public portal are retained by the authors and/or other copyright owners and it is a condition of accessing publications that users recognise and abide by the legal requirements associated with these

- Users may download and print one copy of any publication from the public portal for the purpose of private study or research.

- You may not further distribute the material or use it for any profit-making activity or commercial gain

If the publication is distributed under the terms of Article $25 \mathrm{fa}$ of the Dutch Copyright Act, indicated by the "Taverne" license above, 
Dietary acrylamide intake and human cancer risk 
Dietary acrylamide intake and human cancer risk Janneke Hogervorst

ISBN 9789052788463

Cover graphic: Bas Verhage

Lay-out: Janneke Hogervorst

Printed by: Datawyse - Universitaire Pers Maastricht

(C) Copyright Janneke Hogervorst, Maastricht 2009

Printed on $100 \%$ post-consumer recycled paper

All rights reserved. No part of this thesis may be reproduced or transmitted in any form or by any means, electronic or mechanical, including photocopying, recording or any information storage or retrieval system without permission in writing from the author, or, when appropriate, from the publisher of the publications. 


\section{Dietary acrylamide intake and human cancer risk}

\section{PROEFSCHRIFT}

ter verkrijging van de graad van doctor aan de Universiteit Maastricht, op gezag van de Rector Magnificus,

Prof. mr. G.P.M.F. Mols, volgens het besluit van het College van Decanen, in het openbaar te verdedigen op vrijdag 2 oktober 2009 om 14:00 uur

door

Janneke Greetje Fransje Hogervorst

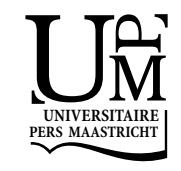




\section{Promotor}

Prof. dr. ir. P.A. van den Brandt

\section{Copromotores}

Dr. L.J. Schouten

Dr. E.J.M. Konings, Voedsel en Waren Autoriteit, Eindhoven

\section{Beoordelingscommissie}

Prof. dr. J.C.S. Kleinjans (voorzitter)

Prof. dr. Ph. Lambin

Dr. L.A. Mucci, Harvard School of Public Health, Boston USA

Dr. A. Romano

Prof. dr. E.G. Schouten, Voedsel en Waren Autoriteit, 's-Gravenhage

This Ph.D. research was supported by the Food and Consumer Product Safety Authority (Voedsel en Waren Autoriteit).

The studies presented in this thesis were conducted at the Department of Epidemiology, School for Oncology and Developmental Biology (GROW), Maastricht University.

Financial support for the printing of this thesis was kindly provided by the Stichting Nationaal Fonds tegen Kanker voor onderzoek naar reguliere en aanvullende therapieën in Amsterdam and the Department of Epidemiology (Maastricht University). 



\section{Contents}

Chapter 1 Introduction 9

Chapter 2 Variability of acrylamide concentrations in single foods does not preclude assessing dietary acrylamide intake through questionnaires

Chapter 3 A prospective study of dietary acrylamide intake and the risk of endometrial, ovarian, and breast cancer

Chapter 4 Dietary acrylamide intake and the risk of renal cell, bladder, and prostate cancer

Chapter 5 Dietary acrylamide intake is not associated with gastrointestinal cancer risk

Chapter 6 Lung cancer risk in relation to dietary acrylamide intake

Chapter 7 Dietary acrylamide intake and brain cancer risk

Chapter 8 Dietary acrylamide intake and the risk of head-neck and thyroid cancer: results from the Netherlands Cohort Study

Chapter 9 The carcinogenicity of dietary acrylamide intake: a side by side discussion of epidemiological and toxicological research

Chapter 10 Epilogue

Summary

Samenvatting

Dankwoord

About the author 



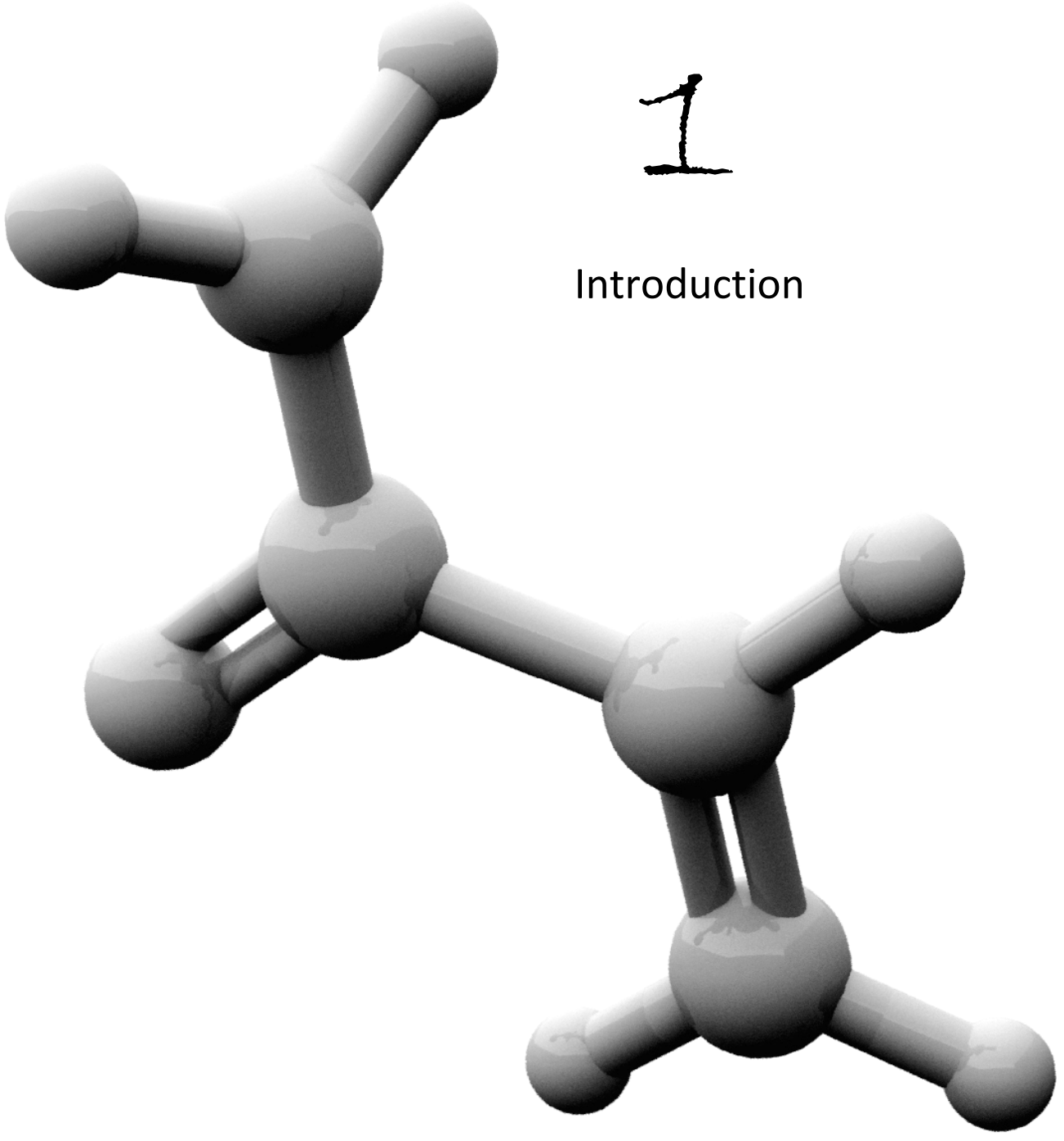


Cancer has become the most important cause of death in the Netherlands in the past year as calculated by Statistics Netherlands (1), and will become the most important cause of death throughout the whole world in the near future (2). In the Netherlands, 1 out of 2.3 men will develop cancer at some point in his life, whereas the corresponding chance for women is 1 out of 2.6 (3). Similar probabilities apply to other Western countries, such as the USA (4). Richard Doll estimated in 1998 that diet-related factors were responsible for $20-50 \%$ of the cancer cases (5).

In 2002, acrylamide, a known rodent carcinogen, was detected in common foods, such as coffee, French fries, potato crisps and cookies. Due to its ubiquitous presence in foods and concentrations of up to $3 \mathrm{mg} / \mathrm{kg}$ in foods, acrylamide might be responsible for a considerable part of the diet-related cancer cases, according to some researchers involved in acrylamide research $(6,7)$. However, their estimates were based on extrapolation of high dose exposure in rats to low dose exposure in humans, and therefore surrounded by considerable uncertainty. Epidemiological studies can help to reduce this uncertainty.

The topic of this thesis is the human cancer risk posed by dietary intake of acrylamide. It describes the results of epidemiological analyses of the association between dietary acrylamide intake and the risk of several types of cancer in a prospective cohort study.

\section{Acrylamide characteristics}

Acrylamide (2-propenamide, $\mathrm{C}_{3} \mathrm{H}_{5} \mathrm{NO}$ ) is a white, odourless and crystalline solid at room temperature, with a molecular weight of $71.08 \mathrm{~g} / \mathrm{mole}$. It is readily dissolvable in water, and other polar solvents, such as ethanol and chloroform. Its chemical structure is shown in Figure 1.

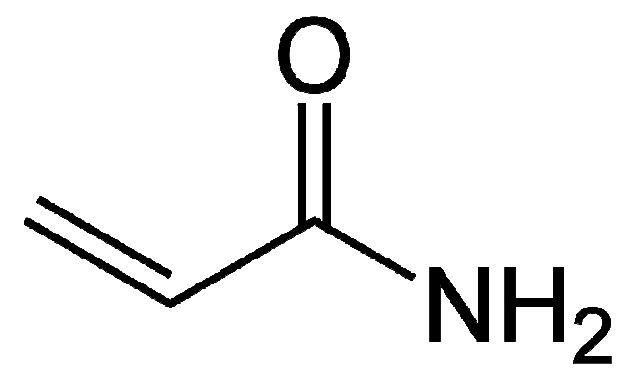

Figure 1: Chemical structure of acrylamide

Due to it being an $\alpha, \beta$-unsaturated carbonyl derivative (i.e. an electron-withdrawing group, e.g carbonyl or amide, coupled to an alkene), (8) acrylamide reacts readily with other acrylamide molecules (forming polyacrylamide), but also with other molecules, particularly molecules containing sulfhydryl (SH) groups like proteins and amino acids (cysteine). Also amino $\left(\mathrm{NH}_{2}\right)$ groups in proteins (e.g. haemoglobin) are targets for acrylamide alkylation. 


\section{Sources of exposure}

Acrylamide is produced from acrylonitrile in industrial plants since the mid 1950s. It can be polymerised to form polyacrylamide, which is a versatile industrial chemical, used in the production of among others paper, plastics, textile, and paint. Monomeric acrylamide is used to make gels for electrophoresis in biochemical laboratories. Furthermore, grout used in construction of buildings and infrastructure contains acrylamide. Thus, occupation can be a source of acrylamide exposure, mainly through inhalation and dermal contact.

Cigarette smoke contains acrylamide and the estimated exposure resulting from average smoking is approximately $3 \mu \mathrm{g} / \mathrm{kg}$ bodyweight per day (9).

Polyacrylamide is used as a coagulant to purify water in order to make it potable. The polymer itself is not toxic, but there is always some residue of the monomer present in the drinking water, for which drinking water standards have been set. Upper-bound excess lifetime human cancer risks of $10^{-4}, 10^{-5}$ and $10^{-6}$ are estimated to occur at 5, 0.5 and 0.05 $\mu \mathrm{g} / \mathrm{L}$, respectively, which has been calculated based on results from rodent carcinogenicity assays (10). In the European Union, the legal limit for drinking water is $0.1 \mu \mathrm{g} / \mathrm{L}$. Assuming the latter concentration and a daily consumption of 2 liters of water a day, an average person weighing 70 kilograms would receive a dose of $0.003 \mu \mathrm{g} / \mathrm{kg}$ bodyweight per day from drinking water ingestion.

Cosmetics contain some residual monomeric acrylamide due to the fact that polyacrylamide is used for instance in shampoos as a foaming agent and as a lubricant in make-up, soaps, and lotions (11). The median estimated daily dose of acrylamide through the use of cosmetics was assessed to be $4.7^{*} 10^{-5}\left(95^{\text {th }}\right.$ percentile: $\left.7.3^{*} 10^{-4}\right) \mu \mathrm{g} / \mathrm{kg}$ bodyweight per day for women, and $3.6^{*} 10^{-5}\left(95^{\text {th }}\right.$ percentile: $\left.5.4 * 10^{-4}\right) \mu \mathrm{g} / \mathrm{kg}$ bodyweight per day for men (11).

In 2002, the Swedish National Food Agency reported on the presence of acrylamide in commonly consumed foods, such as French fries and potato crisps for the first time. Shortly after, various other laboratories confirmed this finding. Acrylamide was found out to occur in foods because of the Maillard reaction, which is a chemical reaction between amino acids and reducing sugars, occurring at high temperatures ( $>120^{\circ}$ Celsius), and at conditions of low moisture content and low acidity. The Maillard reaction is a form of nonenzymatic browning and, similar to caramelisation (pyrolysis of sugars), it gives flavour to foods. Acrylamide formation specifically is a reaction between the amino acid asparagine and reducing sugars, primarily fructose and glucose (Figure 2). Thus, foods that contain asparagine and sugars (e.g. potatoes and cereals) that are baked, grilled, fried, roasted, etc., are prone to acrylamide formation. For potato products, the amount of reducing sugars in the raw potato is the rate-limiting factor, whereas in cereal products the amount of asparagine is the rate-limiting factor. For both potato and cereal products, the conditions of the food processing, such as temperature, duration of heating and the matrix of the food, also determine the variation in acrylamide concentrations found within and between types of food. In the years after 2002, several national food safety agencies across the world have estimated dietary acrylamide intake, based on national food consumption data and acrylamide in food databases. 


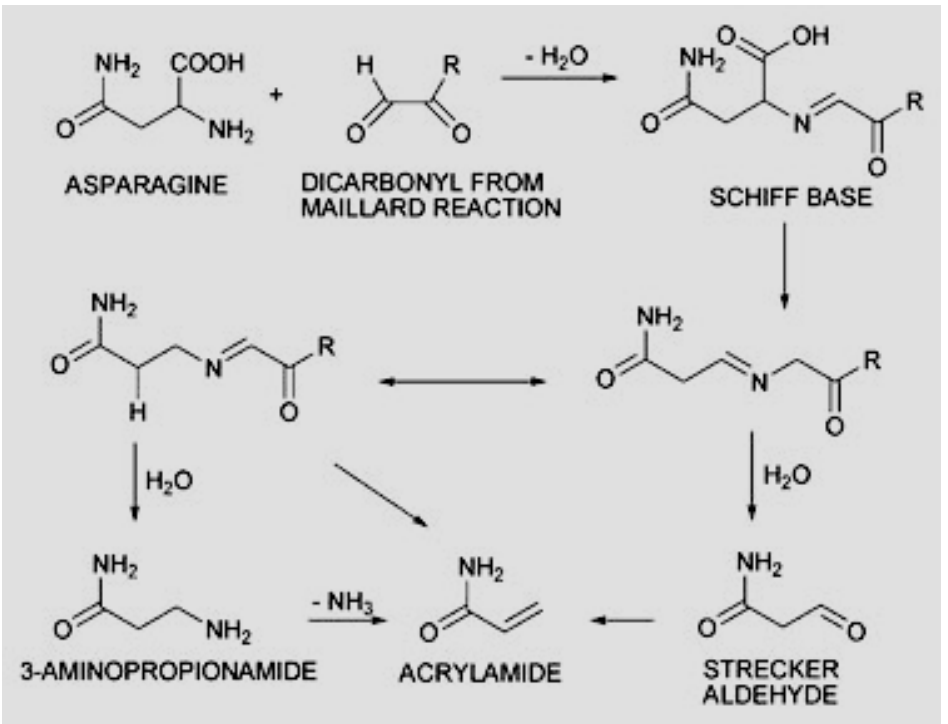

Figure 2: Scheme of the major route of acrylamide formation in food

The average intake across countries is quite similar: the intake of the average person in industrialised countries ranges roughly from 0.3 to $0.8 \mu \mathrm{g} / \mathrm{kg}$ body weight per day (1215). The intake of children per kilogram of bodyweight is estimated to be 1.5 or more times higher than that of adults (15).

From the above it can be derived that for non-occupationally exposed people, the most important sources of exposure are smoking and diet, followed by drinking water and cosmetics.

The observation of acrylamide, a known rodent carcinogen, in commonly consumed foods gave rise to considerable concern, both in the scientific community and the general public. The carcinogenic potency of acrylamide is less than that of several other known food carcinogens, such as polycyclic aromatic hydrocarbons (16), but acrylamide is present at considerably higher concentrations in our foods than those other carcinogens. Based on the estimate made by the World Health Organization and the Norwegian Food Control Authority of the cancer slope factor (an estimate of the extra number of cancer cases per unit of dose based on the animal acrylamide carcinogenicity studies) and the estimated dietary acrylamide intake of the Dutch population, Konings et al. calculated that the cancer risk posed by acrylamide in food would be 3.5 and 6 per ten thousand lifelong exposed people, respectively (12). Petersen et al. calculated that the cancer risk posed by acrylamide in food would be 19.2 per ten thousand lifelong exposed people (16), based on the estimate made by the US Environmental Protection Agency (EPA) of the cancer slope factor (an estimate of the extra number of cancer cases per unit of dose based on the animal acrylamide carcinogenicity studies) and the estimated dietary acrylamide intake. In light of the maximum risk from a carcinogen that is considered acceptable (one in ten 
thousand to one in a million lifelong exposed), the concern over acrylamide in food is quite legitimate.

\section{Kinetics and dynamics}

Pure acrylamide is readily absorbed into the body when ingested or inhaled and partly (approximately 25\%) upon application to the skin (17). In man, acrylamide is also systemically available upon intake of acrylamide through drinking water (18). A study on healthy male volunteers investigated the uptake of acrylamide in an aqueous solution and found that from 88 to $96 \%$ (dependent on the administered dose) of the acrylamide dose was taken up (19). In rats, the bioavailability of acrylamide in food was $32-44 \%$, whereas the uptake after aqueous gavage was $60-98 \%$ (20). In mice, the corresponding percentages were $23 \%$ for food and $32-52 \%$ for the aqueous solution (21). At least some of the acrylamide in food is absorbed by humans, as shown by small studies with healthy human volunteers (22-24). It has been observed that proteins in food impair the uptake of acrylamide from food into human colorectal cells (25).

Regardless of the route of exposure, acrylamide appears to be rapidly distributed to most tissues in animals (26-28). Acrylamide passes the placenta and distributes evenly over the foetus (27), and is also present in human breast milk (29).

Calleman et al., in their experiments on rats, discovered that absorbed acrylamide was partly converted to the epoxide metabolite glycidamide through oxidation (30). The part that is not oxidised, and also glycidamide, are eliminated to a great extent in urine in the form of mercapturic acids; $\mathrm{N}$-acetyl-S-2-carboxamidoethylcysteine, derived from a reaction product of acrylamide and glutathione, and N-acetyl-S-(3-amino-2-hydroxy-3oxopropyl)cysteine and $\mathrm{N}$-acetyl-S-(1-carbamoyl-2-hydroxyethyl)cysteine, which are derived from the reaction of glycidamide and glutathione $(31,32)$. Glycidamide is partly hydrolysed to glyceramide (32), for instance by epoxide hydrolases. Some of the acrylamide and glycidamide is excreted in urine in unchanged form. The conversion of acrylamide to glycidamide has been shown to be probably almost exclusively caused by cytochrome P450-2E1 (CYP2E1) (33-36). There are some species differences in the efficiency of the steps in acrylamide metabolism. Of the investigated species, mice have the highest efficiency of acrylamide to glycidamide conversion, followed by rats and then humans (19, 37). In rats, $28 \%$ of an acrylamide dose of $3 \mathrm{mg} / \mathrm{kg}$ body weight was detected in urine as glycidamide-derived metabolites, whereas in humans this was $12 \%$ at the same dose (18). The ratio of glycidamide to acrylamide haemoglobin adducts in humans was 0.44 in humans and 0.84 in rats (18). Thus, in rats the conversion of acrylamide to glycidamide is about twice as efficient as that in humans, but qualitatively the same metabolism appears to apply to rats and humans. Further downstream in the metabolism, in humans considerably more of the glycidamide is metabolised to glyceramide through hydrolysis than through conjugation with glutathione, as compared with rodents (19). Figure 3 depicts the metabolism of acrylamide. 
As already discussed above, acrylamide has the potential to bind (alkylate) nucleophilic sites, such as sulfhydryl groups and amino groups in amino acids and proteins. Its epoxide metabolite glycidamide also alkylates nucleophilic sites (17), but has a lower reactivity toward sulfhydryl groups than acrylamide itself (38). Glycidamide, however, has been shown to form adducts with DNA amino groups $(28,39)$, whereas acrylamide itself has a low reactivity toward DNA (40), as further discussed below. Both acrylamide and glycidamide bind to haemoglobin in red blood cells, and those adducts are considered to be a biomarker of internal dose over the approximately 4-month lifetime of erythrocytes (9). In the body, acrylamide could have all sorts of effects, depending on which protein it alkylates, whether the alkylation changes the tertiary structure of the protein, and the role of the protein in a certain physiological process (41).

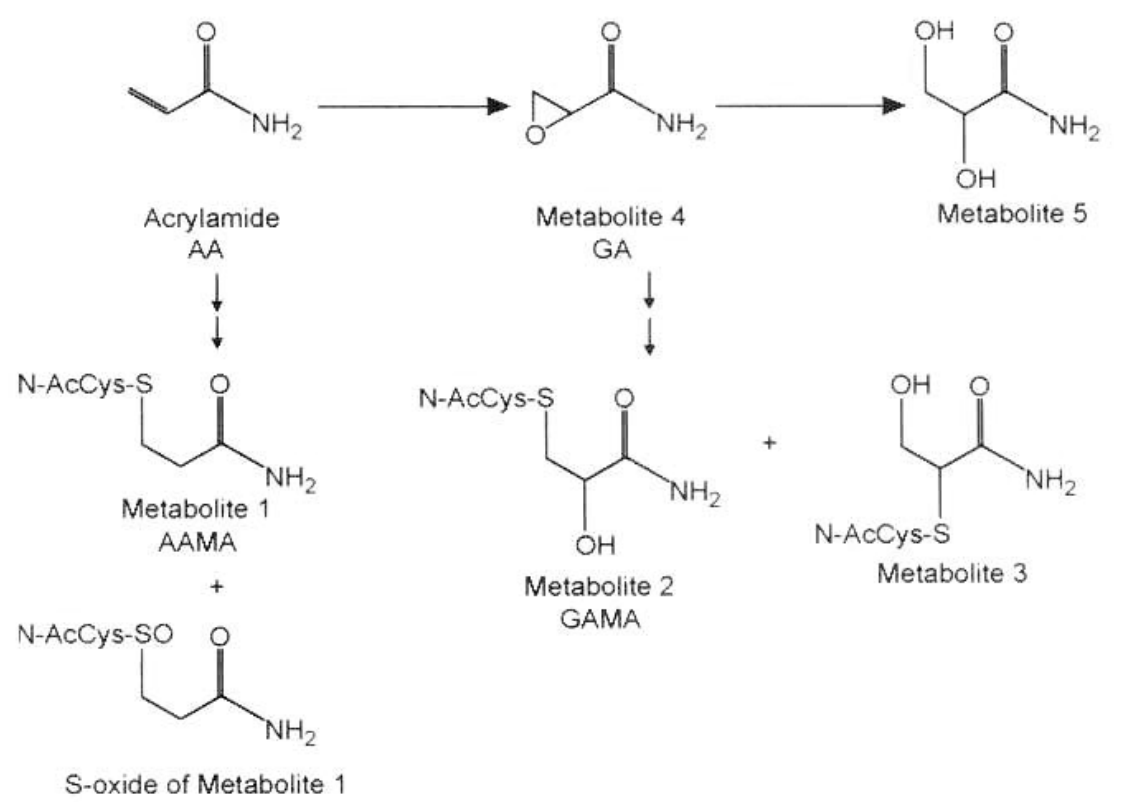

Figure 3: Metabolism of acrylamide

Metabolites: 1 = N-acetyl-S-carboxamidoethylcysteine, $2=\mathrm{N}$-acetyl-S-(3-amino-2-hydroxy-3oxopropyl)cysteine, 3 = N-acetyl-S-(1-carbamoyl-2-hydroxyethyl)cysteine, 4 = glycidamide, 5 = glyceramide

\section{Carcinogenicity}

In 1994, the International Agency for Research on Cancer (IARC) classified acrylamide as a group 2A carcinogen (42), which means that it is deemed a probable human carcinogen. It is the next highest level under level 1: human carcinogen, for which there must be sufficient evidence of carcinogenicity in humans. The 2A category is used when there is limited evidence of carcinogenicity in humans, but sufficient evidence of carcinogenicity in ex- 
perimental animals. In some cases, an agent (mixture) may be classified in this category when there is inadequate evidence of carcinogenicity in humans and sufficient evidence of carcinogenicity in experimental animals and strong evidence that the carcinogenesis is mediated by a mechanism that also operates in humans.

The US Environmental Protection Agency (EPA) has a slightly different classification system for carcinogens. In their system, acrylamide is a B2 carcinogen, which is also a probable human carcinogen. However, the EPA distinguishes between B1 and B2 carcinogens: B1: agents for which there is limited evidence of carcinogenicity from epidemiological studies and sufficient evidence from animal studies, and B2: agents for which there is sufficient evidence from animal studies and for which there is inadequate evidence or no data from epidemiological studies.

Below, the evidence for the carcinogenicity of acrylamide from different research fields (recently reviewed by Besaratinia et al, 2007 and Exon, 2006) $(43,44)$ is shortly described.

\section{Experimental animal research on carcinogenicity}

There have been two chronic Fischer 344 rat carcinogenicity studies so far, and another study from the National Toxicology Program is currently underway (45). The daily doses tested in those two studies range from twenty times to six thousand times the amount that humans in industrialised countries generally take in through their diets (which is on average approximately $0.5 \mu \mathrm{g} / \mathrm{kg}$ body weight per day). The first study showed increased tumour incidence in the mammary gland, central nervous system, thyroid gland (follicular epithelium), oral tissues, uterus, pituitary gland (benign adenoma), and clitoral gland in female rats, and thyroid gland (benign adenoma), oral tissue (epithelial hyperplasia), adrenal gland (benign pheochromocytoma), and scrotal mesothelium in male rats (46). In the second study, these results were reproduced only for the thyroid gland in both males and females, for scrotal mesothelium in males, and for mammary gland adenocarcinomas and fibroadenomas in females (47).

Studies in mice have shown increased incidences of skin and lung tumours, but mostly only after a promoting agent was given after acrylamide exposure $(48,49)$. The animal research is described more extensively in Chapter 9 of this thesis.

\section{Epidemiological research on carcinogenicity in humans}

In this section, only the epidemiological research that was published before March 2005 is discussed, in order to illustrate the state of affairs at the start of the study described in this thesis. In Chapter 9, all the epidemiological evidence of the association between dietary acrylamide intake and cancer risk that has been published up to March 2009 is discussed in conjunction with the results from this thesis.

There were some studies on cancer risk of occupational acrylamide exposure and some studies on dietary acrylamide exposure. The studies on humans with occupational acrylamide exposure showed no increased risks, apart from an increased risk of pancreatic cancer, but this was based on a small number of cases $(50,51)$. In addition, an increased risk of respiratory tract cancer was observed $(50,52)$, but the authors ascribed this finding 
to concurrent exposure to organic dyes. In addition, there was no evidence of a doseresponse relationship, which does not support a causal association between acrylamide exposure and respiratory tract cancer risk. Furthermore, these occupational studies lacked appropriate control for confounders, such as smoking, which further complicates the interpretation of the results.

Up to March 2005, there were only three studies on dietary acrylamide intake and cancer risk, which were two case-control studies $(53,54)$ and one prospective cohort study (55). In these studies, no positive association was found between dietary acrylamide intake and the risk of kidney, renal cell, bladder, colorectal and breast cancer.

The statistically significant inverse association that was observed for colorectal cancer was possibly due to confounding by fibre (53). However, these studies had some limitations. In the case-control study on kidney, bladder and colorectal cancer (53), acrylamide intake assessment was based on frequencies of consumption of acrylamide-containing foods and a ranking number for foods based on their average acrylamide content (rather than the average content itself). Further, acrylamide from coffee was not included in the acrylamide intake assessment. In the case-control study on renal cell cancer (54), acrylamide values for individual foods were not only based on foods on the Swedish market, but also originated from a database on foods in the United States of America. These latter foods are possibly not representative of the Swedish foods. Furthermore, case-control studies are prone to bias, because one first selects cases and controls that are then queried about their dietary habits. In the selection process of cases and controls, bias can occur (selection bias) and information bias can occur due to the fact that cases may have changed their dietary habits due to their disease or recall their dietary habits differently than the controls (recall bias).

\section{Mechanisms of carcinogenicity}

\section{Genotoxicity}

Acrylamide itself is not a bacterial mutagen, but glycidamide is (56-58). Glycidamide forms DNA adducts at guanine (N7) and adenine (N1) positions in DNA both in vitro and in vivo, but acrylamide itself does not $(28,39)$.

In vitro, clastogenic effects of acrylamide have been observed (e.g., in human lymphocytes and hepatocytes), among which were sister chromatid exchanges, micronuclei, mitotic disturbances and single strand breaks $(56,57,59-61)$. In vivo, clastogenic effects were observed in mouse bone-marrow cells, splenocytes, and peripheral blood erythrocytes (micronuclei in the latter cells) $(56,62-68)$.

In vitro, acrylamide induced DNA mutations at the thymidine kinase (tk) and the hypoxanthine-guanine phosphoribosyltransferase (hprt) locus in mouse lymphoma cells (56, 57, 59), at the CII transgene and the TP3 gene in mouse fibroblasts (69) and the hprt locus in Chinese hamster V-79 cells (60). In vivo, DNA mutation frequencies were increased in the Big blue mouse: $G: C-->T: A$ transversions and $-1 /+1$ frameshifts $(70)$ and $A>G, G>C$, and $\mathrm{G}>\mathrm{T}$ transversions (71). Also in germ cells, DNA mutations were observed $(72,73)$. Both in 
vitro and in vivo acrylamide induced DNA breakage and (74) micronuclei formation in germ cells (spermatoctyes, spermatids and spermatogonia) $(75,76)$. In mice and rats, acrylamide caused dominant lethal mutations $(77,78)$.

Aneuploidy was induced by acrylamide in Chinese hamster cells $(57,79)$, and in vivo in mouse spermatocytes (79). Unscheduled DNA synthesis upon acrylamide exposure was seen in rat spermatocytes in vivo (80).

It appears that the genotoxic effect of acrylamide is more of a clastogenic than a mutagenic nature and the genotoxicity seems to primarily be an effect of glycidamide, not acrylamide itself $(35,70,81,82)$.

\section{Non-genotoxic effects}

There are a number of non-genotoxic effects that might be involved in acrylamide carcinogenesis, some of them causing secondary genotoxicity. Oxidative stress, following depletion of glutathione by acrylamide is one of the proposed mechanisms $(61,83,84)$. The resulting change of the redox status in cells can lead to altered gene expression.

Cell transformation in Syrian Hamster Cells, which mimics the multistage properties of carcinogenicity, was increased upon acrylamide exposure (effect of acrylamide itself, not glycidamide), but concurrent exposure to $\mathrm{N}$-acetyl-L-cysteine reduced the acrylamideinduced cell transformation (43).

The aneuploidy that was observed in some test systems upon acrylamide incubation might be caused by binding of acrylamide to proteins involved in cell division, such as mitotic/meiotic spindle kinesins $(85,86)$.

Acrylamide has been shown to influence hormone levels in rodents, among which were prolactin, testosterone, thyroid hormones, and the dopamine system, and to increase the expression of genes involved in the generation of sex hormones in human breast and colorectal cells (87-92). A hormonal effect corresponds to the pattern of tumour formation that was observed in rats.

\section{Other health effects}

\section{Neurotoxicity}

Acrylamide is a neurotoxicant in animals (42) and has also been shown to be a neurotoxicant in humans. In fact, it is the only health effect of acrylamide that has been observed in humans so far $(93,94)$. Subchronic exposure of workers (average exposure $30 \mu \mathrm{g} / \mathrm{kg}$ bodyweight per day) to acrylamide resulted in reversible peripheral neuropathy (ataxia, tingling, weakness and numbness in limbs). Longer exposure resulted in more severe symptoms including cerebellar dysfunction followed by neuropathy. Chronic exposure of laboratory animals resembles the neurotoxicity in humans $(17,95)$.

The no observed adverse effect level (NOAEL) in rats for peripheral nerve damage in rats upon subchronic acrylamide exposure was $0.2 \mathrm{mg} / \mathrm{kg}$ bodyweight per day (96).

Interestingly, the NOAEL for light, mostly reversible, neurotoxic symptoms in workers with acrylamide exposure is about $10 \mu \mathrm{g} / \mathrm{kg}$ bodyweight per day, which is considerably 
lower than the rodent NOAEL (94). Neurotoxicity is not thought to occur at exposure levels that are reached through dietary acrylamide intake. Nevertheless, some researchers are worried about long-term intake in humans at current dietary doses. Cumulative effects may occur, since the same neurotoxic effects can be seen at low and high doses of acrylamide with the low doses just requiring longer exposure (44). LoPachin and Gavin argue that the proposed synaptotoxicity (due to acrylamide interfering with proteins at the nerve terminal) may imply that acrylamide is a risk factor for Alzheimer's disease and other neurodegenerative diseases (96).

\section{Reproductive toxicity}

Acrylamide causes testicular atrophy, reduced sperm motility, impaired fertility, disruptions in mating, and reduced litter size in mice and rats. Reproductive effects appear to be limited to male rodents (96). The lowest observed effect level and no observed effect level are 2.0 and $0.5 \mathrm{mg} / \mathrm{kg}$ day, respectively $(17,95,97,98)$. Part of the reproductive effects is thought to be secondary to neurotoxic effects (96). Although reproductive effects at human dietary doses are not anticipated, cumulative effects may occur when the exposure is chronic.

\section{Developmental toxicity}

Exposure of the pregnant rat results in decreased fetal weight, decreased weight at birth and decreased weight gain during lactation, but only at exposure levels that caused maternal toxicity. The lowest observed effect level for developmental toxicity is 20 $\mathrm{mg} / \mathrm{kg} /$ day $(17,95,97,98)$.

Interestingly, there may be cross-links between the different types of acrylamideinduced health effects. Above, it was already mentioned that the effects on the male reproductive system of rats are partly due to neurotoxic effects. For all three cancer types that were caused by acrylamide exposure in both chronic rat studies it has been suggested that a possible mode of action involves an effect of acrylamide on the neurohormonal system (99).

\section{Carcinogenic risk assessment}

Because of acrylamide's presumed genotoxic mechanism of carcinogenesis, linear extrapolation from high chronic doses in rats to low chronic doses in humans is applied in acrylamide risk assessment. Acrylamide risk assessment is based on the chronic carcinogenicity assays in rats. By assuming an intake of $1 \mu \mathrm{g}$ acrylamide/kg body weight per day the lifetime human cancer risk has been calculated to be 0.7 per 1000 by the World Health Organization, 4.5 per 1000 by the U.S. EPA, and 10 per 1000 by the research group of Granath et al. at Stockholm University (100). Combined with current human dietary exposure in industrialised countries, these so-called cancer slope factors would imply unacceptable numbers of extra cancer cases, as already mentioned in the first part of this introduction Chapter. 
The cancer slope factors vary due to, for example, the choice of the cancer endpoint and the starting point (point of departure) of the extrapolation that were used. For example, Dybing and Sanner based their assessment on the tumour that was statistically significantly induced at the lowest dose (101), whereas the EPA summed up all tumours (CNS, thyroid gland, mammary glands, uterus and oral cavity) for the female rat, because the female was the most sensitive of the sexes, as indicated by acrylamide-induced cancer in more tissues than in the male rat (102). As a point of departure for the risk extrapolation to low doses, in the United States the LED10 is often used; the lower boundary of the $95 \%$ confidence interval for the dose that increases tumour incidence by $10 \%$ over background rates. In Europe, the T25 is often used, which is the dose that causes tumours in $25 \%$ of the animals corrected for the background rate (103-106). Some use the benchmark dose (BMD) approach, which is a more advanced method to analyse dose-response data from studies on experimental animals. It is a statistical integration of all available dose-response data, modelling these data into a mathematical fit, and calculating the confidence limits (e.g. the $95 \%$ confidence interval) of this fit. Next, the dose with a significant adverse and critical effect, e.g., a $10 \%$ increase of the cancer incidence (the $\mathrm{BMD}_{10}$ ) is calculated, and finally the lower limit of its confidence interval (the BMD lower confidence limit, e.g. the $95 \%$ confidence limit - $\mathrm{BMDL}_{10}$ ) is taken as the point of departure for deriving a healthbased limit value (103-105). Furthermore, most risk assessments are based on additivity (excess tumours over the background tumour rate in rats), whereas Granath et al. used a multiplicative risk model (ratio between number of tumours at a certain dose and the number of background tumours in rats) (107).

A tool for risk managers to set priorities with respect to which of many possible risks should be managed first is to calculate the margin of exposure (MOE). The MOE is defined as a point of departure on the dose-response curve in animals (e.g. $\mathrm{BMDL}_{10}$ or T25) (103) divided by the estimated intake by humans. In 2005, the Joint FAO/WHO Expert Committee on Food Additives (JECFA) calculated MOEs for the general population (average dietary acrylamide intake: $1 \mu \mathrm{g}$ acrylamide/kg bodyweight per day), which was 300, and for persons with a high ( $4 \mu \mathrm{g} / \mathrm{kg}$ bodyweight per day) acrylamide intake, which was 75 . For a carcinogenic effect, these MOEs are too low to be acceptable. In general, a MOE for carcinogenic effects should be in the order of magnitude of at least 10,000 in order to ensure a reasonable margin of safety between the lowest dose that significantly increased tumour incidence in an animal experiment and the dose representing an acceptable risk for humans (108).

The European Union Scientific Committee on Food has refrained from executing a quantitative acrylamide risk assessment based on extrapolation from high doses in rats to low doses in humans, because in their opinion there are too many uncertainties involved. Instead, they suggest that the "as low as reasonably achievable" (ALARA) principle is applied to acrylamide exposure. To better understand the implications of acrylamide in food for human health, the Committee stresses the need for epidemiological studies to study the acrylamide-cancer link (100). 


\section{Rationale and aims of this thesis}

As shown in the previous section on carcinogenicity, epidemiological research on acrylamide was still scarce at the start of the research described in this thesis (March 2005), and there was no convincing evidence from these epidemiological studies that acrylamide was indeed the human carcinogen that it was presumed to be, at least not at current dietary exposure levels. However, as described, these studies had limitations, such as limitations in the estimation of dietary acrylamide intake, selection and information bias, and inadequate correction for confounders.

Within the rank ordering of epidemiological studies, the prospective cohort study design ranks highest only under the experiment in terms of validity and thus in the feasibility of inferring causality from a study. An experiment in which the experimental group is given acrylamide and the control group a placebo in order to study if the experimental group has a higher cancer incidence would be ethically unacceptable. Compared with the case-control study, which is the design of most of the studies that were done up to March 2005 , a prospective cohort study has a large advantage in terms of validity. The sequence of observations in a prospective study follows the natural sequence of events, which is the only criterion of the Hill criteria for causality that is unequivocal. That is, one starts off with a disease-free cohort in which the dietary acrylamide intake is measured, and one then follows-up this cohort in order to detect cohort members that develop cancer in the follow-up period. This reduces the likelihood of selection and information bias.

Other advantages of the study described in this thesis for studying the link between acrylamide and cancer risk are considerable numbers of cancer cases per cancer type and the possibility to look at cancer types that were implicated by the animal studies and that had not been studied before in humans (such as endometrial, oral cavity and lung cancer), ample opportunities to correct for confounding, and a more elaborate assessment of individual dietary acrylamide intake (see section Study design and methods).

In conclusion, our study could make a substantial contribution to the epidemiological body of evidence on the link between dietary acrylamide intake and human cancer risk.

We aimed to study the association between dietary acrylamide intake and the risk of female reproductive cancer (endometrial, ovarian, breast), urogenital cancer (kidney, bladder, prostate), gastrointestinal cancer (colorectal, stomach, oesophageal, pancreatic), lung, brain, head/neck and thyroid cancer. These cancer types were selected based on indications for a specific cancer site from the animal carcinogenicity studies (and the endocrine-related pattern that emerged from these studies) and the epidemiological studies on occupational acrylamide exposure. The fact that acrylamide diffuses throughout the whole body was the reason that we studied for instance not only pancreatic cancer (that we were interested in because of the occupational study), but also other cancer types within the gastrointestinal system that were not selected based on the animal experiments and occupational exposure studies. 


\section{Study design and methods}

\section{Study design}

The associations between dietary acrylamide intake and cancer risk that are described in this thesis were studied in an observational study, specifically a prospective cohort study. In short, the exposure of a cohort (free from the disease under study) is measured at baseline. Then, the cohort (or a subcohort from this cohort, as described below) is followed-up in time to detect incident cancer cases. Subsequently, cancer incidence rates (number of incident cancer cases divided by the person time at risk) can be calculated for different categories of exposure. Then, measures of association can be calculated, such as the hazard ratio, which is the ratio between the incidence rate in a certain category of exposure and the incidence rate in a non-exposed or (in the case of dietary acrylamide intake) less exposed category of exposure.

The observational research described in this thesis took place within the framework of the Netherlands Cohort Study on diet and cancer (NLCS) (109). A schematic presentation of the design of this study is shown in Figure 4.

\section{NLCS STUDY DESIGN}

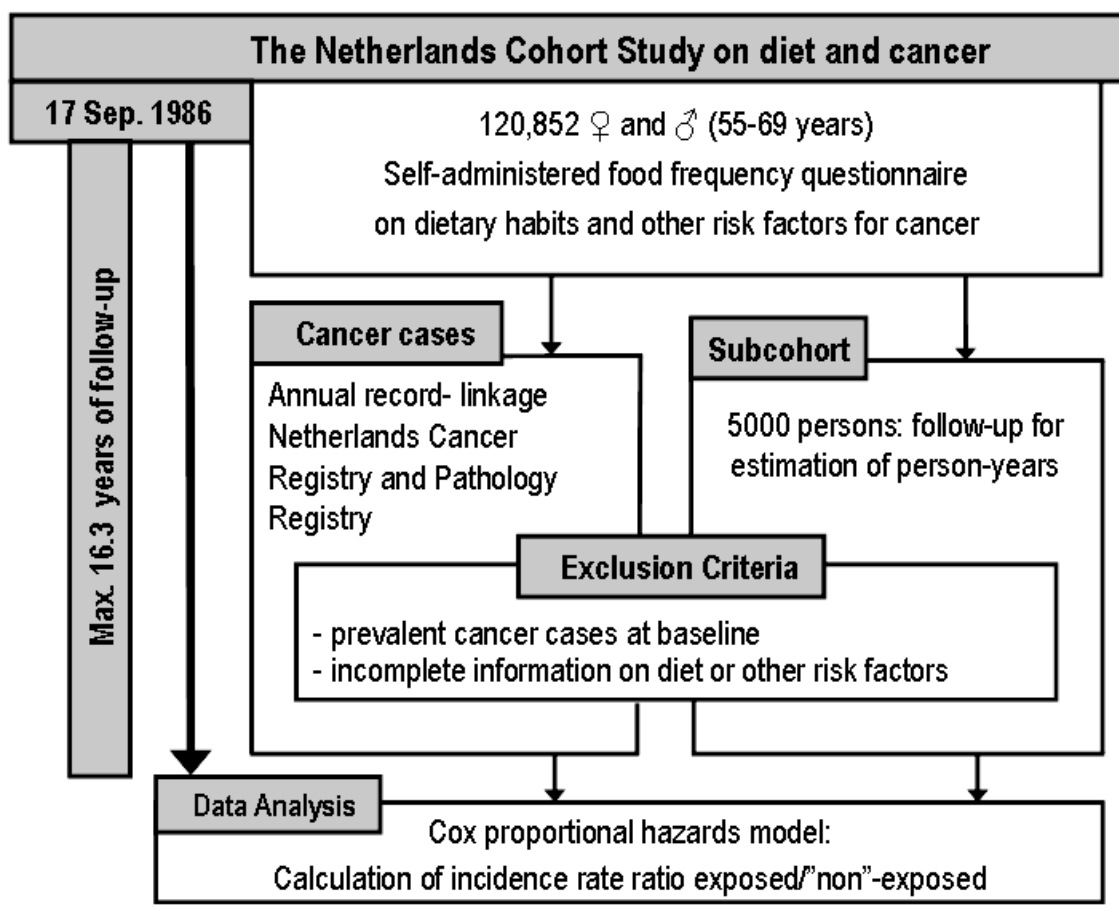

Figure 4: Schematic representation of the study design of the Netherlands Cohort Study on diet and cancer (NLCS) 
Data processing and analysis were based on the case-cohort approach, in which the cases were enumerated for the entire cohort (providing the numerator information for estimating incidence rates), whereas the accumulated person-years at risk for the entire cohort were estimated from a subcohort $(n=5000)$ randomly sampled from the entire cohort at baseline (providing the denominator information for estimating incidence rates). Thus, all the incident cases (including the ones occurring in the subcohort) were used in the analyses. The subcohort was randomly drawn from the entire cohort at baseline.

The database of the Netherlands Cohort Study is updated repeatedly in terms of follow-up. This means that our analyses on acrylamide and cancer risk are done with followup periods ranging from 11.3 to 16.3 years. Naturally, the number of cancer cases increases as follow-up time increases. For the analyses of more rare types of cancer, such as brain, head/neck and thyroid cancer, we therefore used the database for 16.3 years of follow-up.

\section{Acrylamide intake estimation}

In the NLCS, a food frequency questionnaire with questions on 150 food items was used to estimate dietary habits. The acrylamide intake was estimated from the mean acrylamide level of food items, and the frequency of consumption and portion size of the food items. For the acrylamide intake estimation in the NLCS, we focused on foods that are likely to contain considerable acrylamide concentrations, according to the mechanism of formation and reports from various European countries (110). Thus, the following list of food products was established: French fries, potato crisps, bread and rye bread, cookies, pastry, rusk, crisp bread, chocolate and chocolate milk, nuts, peanut butter, breakfast cereals, and coffee. In order to obtain an intake estimate representative for the Dutch situation, we used data on acrylamide concentrations in food products on the Dutch market only. In 2002, the Dutch Food and Consumer Product Safety Authority analysed acrylamide concentrations in various foods, such as bread, French fries, pastry and cake, Dutch spiced cake, crisps, cornflakes, meat, fish, and several beverages, following an elaborate sampling scheme.

In 2005, another series of foods was analysed to specifically accommodate the estimation of acrylamide intake of the NLCS cohort and this series consisted of bread, rusk, specific types of biscuits, rye bread, crisp bread, pastry and cake, chocolate and chocolate milk, nuts and salty snacks, peanut butter, and coffee. Acrylamide measurements in cookies were done in several types of cookies known to be eaten most frequently by a population comparable to the NLCS, according to information from the development phase of the NLCS questionnaire. Thus, the acrylamide level assigned to cookies was based on the acrylamide level of the specific types of cookies weighted by the frequency of consumption of the NLCS-comparable population. The same was done for other composite food items, such as pies and chocolates.

The foods listed above were assigned the mean value of the acrylamide values per food or a value half the quantitation limit when concentrations were lower than the quantitation limit, because they probably do contain some acrylamide. Other foods (meats, cooked and raw vegetables, dairy products) were assigned the value zero. 
The questionnaire, upon which the acrylamide intake assessment of the subcohort was based, was administered to the total cohort at baseline only. However, the stability of dietary habits of the cohort was assessed by repeated administration (during the first five years of follow-up, annually) of the food frequency questionnaire in a 400-person sample of the subcohort (different sample every year). This exercise showed that the decline of the correlation between the baseline questionnaire and the questionnaire administered after five years of follow-up was 0.07 averaged over all nutrients. As for coffee, which contributes importantly to dietary acrylamide intake, $73 \%$ of the subcohort members reported to drink the same amount of coffee at baseline as they did five years before. With regard to validity, the correlation coefficients between the FFQ and a 9-day dietary record method were 0.77 for carbohydrates and 0.74 for fibre. For the food groups potatoes, bread, and cakes and cookies, Spearman correlation coefficients were $0.74,0.80$ and 0.65 , respectively.

Smokers have been shown to have on average four times higher levels of acrylamidehaemoglobin adducts, which is a marker of internal dose of acrylamide, than non-smokers $(111,112)$. Thus, for smokers, the estimation of dietary acrylamide intake might be blurred by intake via cigarette smoke, which may lead to misclassification of the acrylamide exposure. For this reason and because of the fact that smoking is a very important risk factor for many types of cancer, subgroup analyses were done for never-smokers.

\section{Outline of the thesis}

Chapter 2 describes a study in which an important part of the acrylamide intake assessment (assigning mean acrylamide values to individual foods) is validated. Because of the wide distribution of acrylamide in the body upon exposure, the association between dietary acrylamide intake and the risk of cancer in many organs was studied: endometrial, ovarian and female breast cancer (Chapter 3); renal cell, bladder and prostate cancer (Chapter 4); colorectal, stomach, oesophageal, and pancreatic cancer (Chapter 5); lung cancer (Chapter 6); brain cancer (Chapter 7), and head/neck and thyroid cancer (Chapter 8). In Chapter 9, an overview of the current epidemiological body of evidence for the carcinogenicity is given, along with a discussion of and comparison with the body of evidence of animal studies. Reasons for possible discrepancies between the results from both research fields are discussed. Finally, Chapter 10 is a discussion of the main findings of the NLCS acrylamide research in light of some important strengths and limitations, and recommendations for future research. 


\section{References}

1. http://www.cbs.nl/en-GB/menu/themas/gezondheid-welzijn/publicaties/artikelen/archief/ 2009/2009-2687-wm.htm?Languageswitch=on (accessed on February 3 2009)

2. WHO/IARC. World Cancer Report 2008. Lyon, 2008. (accessed on February 3 2009)

3. Kiemeney LA, Lemmers FA, Verhoeven RH, et al. [The risk of cancer in the Netherlands]. Ned Tijdschr Geneeskd 2008;152:2233-41.

4. http://seer.cancer.gov/statfacts/html/all.html. (accessed on February 3 2009)

5. Doll R. Epidemiological evidence of the effects of behaviour and the environment on the risk of human cancer. Recent Results Cancer Res 1998;154:3-21.

6. Granath F, Tornqvist M. Who knows whether acrylamide in food is hazardous to humans? J Natl Cancer Inst 2003;95:842-3.

7. Mucci LA, Adami HO. The role of epidemiology in understanding the relationship between dietary acrylamide and cancer risk in humans. Adv Exp Med Biol 2005;561:39-47.

8. LoPachin RM, Barber DS, Gavin T. Molecular mechanisms of the conjugated alpha,beta-unsaturated carbonyl derivatives: relevance to neurotoxicity and neurodegenerative diseases. Toxicol Sci 2008;104:23549.

9. Bergmark E. Hemoglobin adducts of acrylamide and acrylonitrile in laboratory workers, smokers and nonsmokers. Chem Res Toxicol 1997;10:78-84.

10. http://www.who.int/water_sanitation_health/dwq/chemicals/acrylamide.pdf. (accessed on February 3 2009)

11. Van Landingham CB, Lawrence GA, Shipp AM. Estimates of lifetime-absorbed daily doses from the use of personal-care products containing polyacrylamide: a Monte Carlo analysis. Risk Anal 2004;24:603-19.

12. Konings EJ, Baars AJ, van Klaveren JD, et al. Acrylamide exposure from foods of the Dutch population and an assessment of the consequent risks. Food Chem Toxicol 2003;41:1569-79.

13. Matthys C, Bilau M, Govaert Y, Moons E, De Henauw S, Willems JL. Risk assessment of dietary acrylamide intake in Flemish adolescents. Food Chem Toxicol 2005;43:271-8.

14. Svensson K, Abramsson L, Becker W, et al. Dietary intake of acrylamide in Sweden. Food Chem Toxicol 2003;41:1581-6.

15. Dybing E, Farmer PB, Andersen $\mathrm{M}$, et al. Human exposure and internal dose assessments of acrylamide in food. Food Chem Toxicol 2005;43:365-410.

16. Petersen BJ, Tran N. Exposure to acrylamide: placing exposure in context. Adv Exp Med Biol 2005;561:6376.

17. Friedman M. Chemistry, biochemistry, and safety of acrylamide. A review. J Agric Food Chem 2003;51:450426.

18. Fennell TR, Friedman MA. Comparison of acrylamide metabolism in humans and rodents. Adv Exp Med Biol 2005;561:109-16.

19. Fennell TR, Sumner SC, Snyder RW, et al. Metabolism and hemoglobin adduct formation of acrylamide in humans. Toxicol Sci 2005;85:447-59.

20. Doerge DR, Young JF, McDaniel LP, Twaddle NC, Churchwell MI. Toxicokinetics of acrylamide and glycidamide in Fischer 344 rats. Toxicol Appl Pharmacol 2005;208:199-209.

21. Doerge DR, Young JF, McDaniel LP, Twaddle NC, Churchwell MI. Toxicokinetics of acrylamide and glycidamide in B6C3F1 mice. Toxicol Appl Pharmacol 2005;202:258-67.

22. Fuhr U, Boettcher MI, Kinzig-Schippers $\mathrm{M}$, et al. Toxicokinetics of acrylamide in humans after ingestion of a defined dose in a test meal to improve risk assessment for acrylamide carcinogenicity. Cancer Epidemiol Biomarkers Prev 2006;15:266-71.

23. Sorgel F, Weissenbacher R, Kinzig-Schippers M, et al. Acrylamide: increased concentrations in homemade food and first evidence of its variable absorption from food, variable metabolism and placental and breast milk transfer in humans. Chemotherapy 2002;48:267-74.

24. Doroshyenko O, Fuhr U, Kunz D, et al. In vivo Role of Cytochrome P450 $2 \mathrm{E} 1$ and Glutathione-S-Transferase Activity for Acrylamide Toxicokinetics in Humans. Cancer Epidemiol Biomarkers Prev 2009;18:433-43.

25. Schabacker J, Schwend T, Wink M. Reduction of acrylamide uptake by dietary proteins in a caco-2 gut model. J Agric Food Chem 2004;52:4021-5.

26. Miller MJ, Carter DE, Sipes IG. Pharmacokinetics of acrylamide in Fisher-344 rats. Toxicol Appl Pharmacol 1982;63:36-44. 
27. Marlowe C, Clark MJ, Mast RW, Friedman MA, Waddell WJ. The distribution of [14C]acrylamide in male and pregnant Swiss-Webster mice studied by whole-body autoradiography. Toxicol Appl Pharmacol 1986;86:457-65.

28. Segerback D, Calleman CJ, Schroeder JL, Costa LG, Faustman EM. Formation of N-7-(2-carbamoyl-2hydroxyethyl)guanine in DNA of the mouse and the rat following intraperitoneal administration of [14C]acrylamide. Carcinogenesis 1995;16:1161-5.

29. Fohgelberg P, Rosen J, Hellenas KE, Abramsson-Zetterberg L. The acrylamide intake via some common baby food for children in Sweden during their first year of life--an improved method for analysis of acrylamide. Food Chem Toxicol 2005;43:951-9.

30. Calleman CJ, Bergmark E, Costa LG. Acrylamide is metabolized to glycidamide in the rat: evidence from hemoglobin adduct formation. Chem Res Toxicol 1990;3:406-12.

31. Dixit R, Seth PK, Mukjtar H. Metabolism of acrylamide into urinary mercapturic acid and cysteine conjugates in rats. Drug Metab Dispos 1982;10:196-7.

32. Sumner SC, MacNeela JP, Fennell TR. Characterization and quantitation of urinary metabolites of $[1,2,3-$ 13C]acrylamide in rats and mice using $13 \mathrm{C}$ nuclear magnetic resonance spectroscopy. Chem Res Toxicol 1992;5:81-9.

33. Sumner SC, Fennell TR, Moore TA, Chanas B, Gonzalez F, Ghanayem BI. Role of cytochrome P450 2 E1 in the metabolism of acrylamide and acrylonitrile in mice. Chem Res Toxicol 1999;12:1110-6.

34. Ghanayem BI, McDaniel LP, Churchwell MI, et al. Role of CYP2E1 in the Epoxidation of Acrylamide to Glycidamide and Formation of DNA and Hemoglobin Adducts. Toxicol Sci 2005;88:311-8.

35. Ghanayem $\mathrm{BI}$, Witt $\mathrm{KL}$, El-Hadri L, et al. Comparison of germ cell mutagenicity in male CYP2E1-null and wildtype mice treated with acrylamide: evidence supporting a glycidamide-mediated effect. Biol Reprod 2005;72:157-63.

36. Ghanayem BI, Witt KL, Kissling GE, Tice RR, Recio L. Absence of acrylamide-induced genotoxicity in CYP2E1null mice: Evidence consistent with a glycidamide-mediated effect. Mutat Res 2005;578:284-97.

37. Sumner SC, Selvaraj L, Nauhaus SK, Fennell TR. Urinary metabolites from F344 rats and B6C3F1 mice coadministered acrylamide and acrylonitrile for 1 or 5 days. Chem Res Toxicol 1997;10:1152-60.

38. Tornqvist M. Acrylamide in food: the discovery and its implications: a historical perspective. Adv Exp Med Biol 2005;561:1-19.

39. Gamboa da Costa G, Churchwell MI, Hamilton LP, et al. DNA adduct formation from acrylamide via conversion to glycidamide in adult and neonatal mice. Chem Res Toxicol 2003;16:1328-37.

40. Solomon JJ, Fedyk J, Mukai F, Segal A. Direct alkylation of 2'-deoxynucleosides and DNA following in vitro reaction with acrylamide. Cancer Res 1985;45:3465-70.

41. Lopachin RM, Decaprio AP. Protein Adduct Formation as a Molecular Mechanism in Neurotoxicity. Toxicol Sci 2005;86:214-25.

42. IARC. Monographs on the evaluation of carcinogen risk to humans: some industrial chemicals. Lyon: International Agency for Research on Cancer, 1994.

43. Besaratinia A, Pfeifer GP. A review of mechanisms of acrylamide carcinogenicity. Carcinogenesis 2007;28:519-28.

44. Exon JH. A review of the toxicology of acrylamide. J Toxicol Environ Health B Crit Rev 2006;9:397-412.

45. Summary Report EFSA scientific colloquium no. 11. Acrylamide carcinogenicity new evidence in relation to dietary exposure. 22-23 May 2008, Tabiano (PR), Italy.

46. Johnson KA, Gorzinski SJ, Bodner KM, et al. Chronic toxicity and oncogenicity study on acrylamide incorporated in the drinking water of Fischer 344 rats. Toxicol Appl Pharmacol 1986;85:154-68.

47. Friedman MA, Dulak LH, Stedham MA. A lifetime oncogenicity study in rats with acrylamide. Fundam Appl Toxicol 1995;27:95-105.

48. Bull RJ, Robinson M, Laurie RD, et al. Carcinogenic effects of acrylamide in Sencar and $A / J$ mice. Cancer Res 1984;44:107-11.

49. Bull RJ, Robinson M, Stober JA. Carcinogenic activity of acrylamide in the skin and lung of Swiss-ICR mice. Cancer Lett 1984;24:209-12.

50. Marsh GM, Lucas L, Youk AO, Schall LC. Mortality patterns among workers exposed to acrylamide: 1994 follow up. Occup Environ Med 1999;56:181-90.

51. Schulz MR, Hertz-Picciotto I, van Wijngaarden E, Hernandez JC, Ball LM. Dose-response relation between acrylamide and pancreatic cancer. Occup Environ Med 2001;58:609.

52. Sobel W, Bond GG, Parsons TW, Brenner FE. Acrylamide cohort mortality study. Br J Ind Med 1986;43:7858. 
53. Mucci LA, Dickman PW, Steineck G, Adami HO, Augustsson K. Dietary acrylamide and cancer of the large bowel, kidney, and bladder: absence of an association in a population-based study in Sweden. Br J Cancer 2003;88:84-9.

54. Mucci LA, Lindblad P, Steineck G, Adami HO. Dietary acrylamide and risk of renal cell cancer. Int J Cancer 2004;109:774-6.

55. Mucci LA, Sandin S, Balter K, Adami HO, Magnusson C, Weiderpass E. Acrylamide intake and breast cancer risk in Swedish women. Jama 2005;293:1326-7.

56. Knaap AG, Kramers PG, Voogd CE, et al. Mutagenic activity of acrylamide in eukaryotic systems but not in bacteria. Mutagenesis 1988;3:263-8.

57. Tsuda H, Shimizu CS, Taketomi MK, et al. Acrylamide; induction of DNA damage, chromosomal aberrations and cell transformation without gene mutations. Mutagenesis 1993;8:23-9.

58. Hashimoto K, Tanii H. Mutagenicity of acrylamide and its analogues in Salmonella typhimurium. Mutat Res 1985;158:129-33.

59. Moore MM, Amtower A, Doerr C, Brock KH, Dearfield KL. Mutagenicity and clastogenicity of acrylamide in L5178Y mouse lymphoma cells. Environ Mutagen 1987;9:261-7.

60. Baum M, Fauth E, Fritzen S, et al. Acrylamide and glycidamide: genotoxic effects in V79-cells and human blood. Mutat Res 2005;580:61-9.

61. Jiang L, Cao J, An Y, Geng C, Qu S, Zhong L. Genotoxicity of acrylamide in human hepatoma G2 (HepG2) cells. Toxicol In Vitro 2007;21:1486-92.

62. Adler ID, Ingwersen I, Kliesch U, el Tarras A. Clastogenic effects of acrylamide in mouse bone marrow cells. Mutat Res 1988;206:379-85.

63. Abramsson-Zetterberg L. The dose-response relationship at very low doses of acrylamide is linear in the flow cytometer-based mouse micronucleus assay. Mutat Res 2003;535:215-22.

64. Abramsson-Zetterberg L, Grawe J, Zetterberg G. Flow cytometric analysis of micronucleus induction in mice by internal exposure to $137 \mathrm{Cs}$ at very low dose rates. Int J Radiat Biol 1995;67:29-36.

65. Paulsson B, Kotova N, Grawe J, et al. Induction of micronuclei in mouse and rat by glycidamide, genotoxic metabolite of acrylamide. Mutat Res 2003;535:15-24.

66. Backer LC, Dearfield KL, Erexson GL, Campbell JA, Westbrook-Collins B, Allen JW. The effects of acrylamide on mouse germ-line and somatic cell chromosomes. Environ Mol Mutagen 1989;13:218-26.

67. Cao J, Beisker W, Nusse M, Adler ID. Flow cytometric detection of micronuclei induced by chemicals in polyand normochromatic erythrocytes of mouse peripheral blood. Mutagenesis 1993;8:533-41.

68. Cihak R, Vontorkova M. Cytogenetic effects of acrylamide in the bone marrow of mice. Mutat Res 1988;209:91-4.

69. Besaratinia A, Pfeifer GP. Weak yet distinct mutagenicity of acrylamide in mammalian cells. J Natl Cancer Inst 2003;95:889-96.

70. Manjanatha MG, Aidoo A, Shelton SD, et al. Genotoxicity of acrylamide and its metabolite glycidamide administered in drinking water to male and female Big Blue mice. Environ Mol Mutagen 2006;47:6-17.

71. Besarati Nia A, Pfeifer GP. Genotoxicity of acrylamide and glycidamide. J Natl Cancer Inst 2004;96:1023-9.

72. Russell LB, Hunsicker PR, Cacheiro NL, Generoso WM. Induction of specific-locus mutations in male germ cells of the mouse by acrylamide monomer. Mutat Res 1991;262:101-7.

73. Ehling UH, Neuhauser-Klaus A. Reevaluation of the induction of specific-locus mutations in spermatogonia of the mouse by acrylamide. Mutat Res 1992;283:185-91.

74. Collins BW, Howard DR, Allen JW. Kinetochore-staining of spermatid micronuclei: studies of mice treated with X-radiation or acrylamide. Mutat Res 1992;281:287-94.

75. Sega GA, Generoso EE. Measurement of DNA breakage in specific germ-cell stages of male mice exposed to acrylamide, using an alkaline-elution procedure. Mutat Res 1990;242:79-87.

76. Shiraishi Y. Chromosome aberrations induced by monomeric acrylamide in bone marrow and germ cells of mice. Mutat Res 1978;57:313-24.

77. Shelby MD, Cain KT, Hughes LA, Braden PW, Generoso WM. Dominant lethal effects of acrylamide in male mice. Mutat Res 1986;173:35-40.

78. Smith MK, Zenick H, Preston RJ, George EL, Long RE. Dominant lethal effects of subchronic acrylamide administration in the male Long-Evans rat. Mutat Res 1986;173:273-7.

79. Adler ID, Zouh R, Schmid E. Perturbation of cell division by acrylamide in vitro and in vivo. Mutat Res 1993;301:249-54.

80. Butterworth BE, Eldridge SR, Sprankle CS, Working PK, Bentley KS, Hurtt ME. Tissue-specific genotoxic effects of acrylamide and acrylonitrile. Environ Mol Mutagen 1992;20:148-55. 
81. Ghanayem BI, Witt KL, Kissling GE, Tice RR, Recio L. Absence of acrylamide-induced genotoxicity in CYP2E1null mice: evidence consistent with a glycidamide-mediated effect. Mutat Res 2005;578:284-97.

82. Generoso WM, Sega GA, Lockhart AM, et al. Dominant lethal mutations, heritable translocations, and unscheduled DNA synthesis induced in male mouse germ cells by glycidamide, a metabolite of acrylamide. Mutat Res 1996;371:175-83.

83. Klaunig JE, Kamendulis LM. Mechanisms of acrylamide induced rodent carcinogenesis. Adv Exp Med Biol 2005;561:49-62.

84. Yousef MI, El-Demerdash FM. Acrylamide-induced oxidative stress and biochemical perturbations in rats. Toxicology 2006;219:133-41.

85. Lapadula DM, Bowe M, Carrington CD, Dulak L, Friedman M, Abou-Donia MB. In vitro binding of [14C]acrylamide to neurofilament and microtubule proteins of rats. Brain Res 1989;481:157-61.

86. Sickles DW, Sperry AO, Testino A, Friedman M. Acrylamide effects on kinesin-related proteins of the mitotic/meiotic spindle. Toxicol Appl Pharmacol 2007;222:111-21.

87. Agrawal AK, Seth PK, Squibb RE, Tilson HA, Uphouse LL, Bondy SC. Neurotransmitter receptors in brain regions of acrylamide- treated rats. I: Effects of a single exposure to acrylamide. Pharmacol Biochem Behav 1981;14:527-31.

88. Ali SF, Hong JS, Wilson WE, Uphouse LL, Bondy SC. Effect of acrylamide on neurotransmitter metabolism and neuropeptide levels in several brain regions and upon circulating hormones. Arch Toxicol 1983;52:3543.

89. Uphouse LL. Interactions between handling and acrylamide on the striatal dopamine receptor. Brain Res 1981;221:421-4.

90. Uphouse LL, Nemeroff CB, Mason G, Prange AJ, Bondy SC. Interactions between "handling" and acrylamide on endocrine responses in rats. Neurotoxicology 1982;3:121-5.

91. Mannaa F, Abdel-Wahhab MA, Ahmed HH, Park MH. Protective role of Panax ginseng extract standardized with ginsenoside Rg3 against acrylamide-induced neurotoxicity in rats. J Appl Toxicol 2006;26:198-206.

92. Clement FC, Dip R, Naegeli H. Expression profile of human cells in culture exposed to glycidamide, a reactive metabolite of the heat-induced food carcinogen acrylamide. Toxicology 2007;240:111-24.

93. Calleman CJ, Wu Y, He F, et al. Relationships between biomarkers of exposure and neurological effects in a group of workers exposed to acrylamide. Toxicol Appl Pharmacol 1994;126:361-71.

94. Kjuus H, Goffeng LO, Heier MS, et al. Examination of nervous system effects and other health effects in tunnel workers exposed to acrylamide and $\mathrm{N}$-methylolacrylamide in Romeriksporten, Norway. Oslo: National Institute of Occupational Health, 2002.

95. Lopachin RM, Gavin T. Acrylamide-induced nerve terminal damage: relevance to neurotoxic and neurodegenerative mechanisms. J Agric Food Chem 2008;56:5994-6003.

96. Tyl RW, Friedman MA. Effects of acrylamide on rodent reproductive performance. Reprod Toxicol 2003;17:1-13.

97. Dearfield KL, Abernathy CO, Ottley MS, Brantner JH, Hayes PF. Acrylamide: its metabolism, developmental and reproductive effects, genotoxicity, and carcinogenicity. Mutat Res 1988;195:45-77.

98. Alexander J. Risk assessment techniques for acrylamide. In: K. Skog and J. Alexander (eds.), Acrylamide and other hazardous compounds in heat-treated foods, pp. 275-295. Boca raton: CRC Press, 2006.

99. Shipp A, Lawrence G, Gentry R, et al. Acrylamide: review of toxicity data and dose-response analyses for cancer and noncancer effects. Crit Rev Toxicol 2006;36:481-608.

100. http://ec.europa.eu/food/fs/sc/scf/out131_en.pdf. (accessed on February 3 2009)

101. Dybing E, Sanner T. Risk assessment of acrylamide in foods. Toxicol Sci 2003;75:7-15.

102. http://www.epa.gov/iris/subst/0286.htm. (accessed on February 3 2009)

103. Dybing E, O’Brien J, Renwick AG, Sanner T. Risk assessment of dietary exposures to compounds that are genotoxic and carcinogenic--an overview. Toxicol Lett 2008;180:110-7.

104. Crebelli R. Towards a harmonized approach for risk assessment of genotoxic carcinogens in the European Union. Ann Ist Super Sanita 2006;42:127-31.

105. O'Brien J, Renwick AG, Constable A, et al. Approaches to the risk assessment of genotoxic carcinogens in food: a critical appraisal. Food Chem Toxicol 2006;44:1613-35.

106. Parzefall W. Minireview on the toxicity of dietary acrylamide. Food Chem Toxicol 2008;46:1360-4.

107. Granath FN, Vaca CE, Ehrenberg LG, Tornqvist MA. Cancer risk estimation of genotoxic chemicals based on target dose and a multiplicative model. Risk Anal 1999;19:309-20.

108. Summary and conclusions of the sixty-fourth meeting of the Joint FAO/WHO Expert Committee on Food Additives (JECFA): JECFA, 2005. 
109. van den Brandt PA, Goldbohm RA, van 't Veer P, Volovics A, Hermus RJ, Sturmans F. A large-scale prospective cohort study on diet and cancer in The Netherlands. J Clin Epidemiol 1990;43:285-95.

110. http://www.irmm.jrc.be/html/activities/acrylamide/database.htm. (accessed on February 3 2009)

111. Schettgen T, Rossbach B, Kutting B, Letzel S, Drexler H, Angerer J. Determination of haemoglobin adducts of acrylamide and glycidamide in smoking and non-smoking persons of the general population. Int J Hyg Environ Health 2004;207:531-9.

112. Vesper HW, Slimani N, Hallmans G, et al. Cross-sectional study on acrylamide hemoglobin adducts in subpopulations from the European Prospective Investigation into Cancer and Nutrition (EPIC) Study. J Agric Food Chem 2008;56:6046-53. 


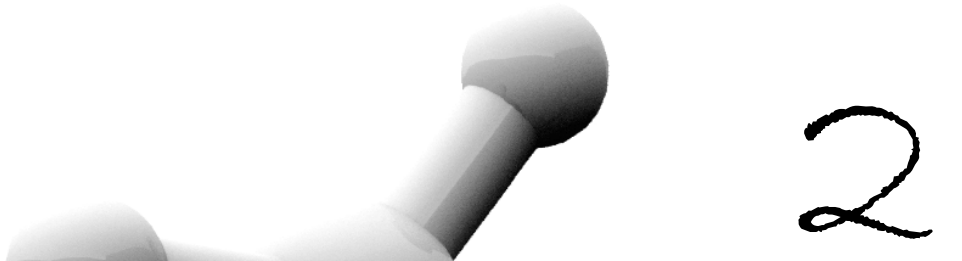

Variability of acrylamide concentrations in single foods does not preclude assessing dietary acrylamide intake through questionnaires

Erik J.M. Konings Janneke G.F. Hogervorst Linda van Rooij Leo J. Schouten Eric A. Sizoo Hans P. van Egmond R. Alexandra Goldbohm Piet A. van den Brandt 


\begin{abstract}
Background Acrylamide, a probable human carcinogen, was detected in various heattreated foods, such as French fries and potato crisps. Recently, positive associations have been found between dietary acrylamide intake, as estimated with a food frequency questionnaire using mean acrylamide concentrations for individual foods, and cancer risk in some epidemiological studies.
\end{abstract}

Objective Because acrylamide concentrations in individual foods vary considerably, a validation study was done to investigate whether using mean acrylamide concentrations in foods, as applied in e.g. the Netherlands Cohort Study on diet and cancer (NLCS), results in a sound estimate of total acrylamide intake.

Design We made use of a 24-hour duplicate diet study. The acrylamide content of 39 Dutch 24-hour duplicate diets collected in 2004 was estimated by using the menu list (on which amounts of individual foods and drinks in the duplicate diets were semiquantitatively listed) that the participants of the duplicate diet study filled in, and mean acrylamide concentrations for foods available from a Dutch database. Next, the acrylamide content of the duplicate diets was analytically measured and correlated to the estimated acrylamide contents.

Results The Spearman correlation coefficient between chemically determined acrylamide content and the estimated acrylamide content of the duplicate diets was $0.82(p<0.001)$.

Conclusions This study indicates that it is feasible to make a sound rank ordering of the acrylamide intake via a 24-hour diet using mean acrylamide concentrations for individual foods, as applied in the epidemiological analyses on acrylamide intake and cancer risk in the NLCS. 


\section{Introduction}

In 2002 , the scientific community was alarmed by the finding by Swedish scientists that acrylamide is present in a wide range of foods, such as French fries and potato crisps (1). In 1994, the International Agency for Research on Cancer classified acrylamide as a probable human carcinogen, based on its carcinogenicity in rodents (2). Acrylamide is present in foods at considerably higher concentrations than other well-known food carcinogens, such as polycyclic aromatic hydrocarbons and ethyl carbamate (3). Shortly after its discovery in food, it was shown that acrylamide is formed in Maillard browning reactions in which amino acids, particularly asparagine, react with reducing sugars at high temperatures $\left(>120^{\circ} \mathrm{C}\right)(4,5)$.

Dietary acrylamide intake and its relation with the risk of various types of cancer in humans have been studied in few case-control studies (6-8) and prospective cohort studies (9-15). In the case-control studies, no association between acrylamide intake and cancer risk was observed. One prospective cohort study by our group, the Netherlands Cohort Study on diet and cancer (NLCS), showed a positive association between dietary acrylamide intake and endometrial cancer risk in never-smokers (11). Furthermore, this study showed a positive association between dietary acrylamide intake and ovarian cancer, but not postmenopausal breast cancer risk (11). The NLCS also provided evidence for a positive relation between dietary acrylamide intake and renal cell cancer risk, especially in individuals who smoke. No such relations were found for bladder and prostate cancer in this study (12). In Denmark, a positive association was found between acrylamide intake and estrogen receptor-positive breast cancer risk among postmenopausal women, using acrylamide to haemoglobin adducts as the exposure variable (13). The other prospective cohort studies have not found indications for positive relationships.

In these studies, most often food frequency questionnaires (FFQs) have been used to assess dietary acrylamide intake. Although the use of FFQs has limitations (16), they are the only feasible way to assess dietary intake over a long period in large-scale epidemiological studies. Two aspects are important for the feasibility to assess dietary acrylamide intake, namely the validity of the FFQ with respect to the relevant acrylamide-containing foods and the ability of the FFQ to capture long-term intake of these foods, and the analytical acrylamide data available for these foods.

Acrylamide concentrations vary enormously not only between foods, but also within foods. For instance, Konings et al. (17) have found acrylamide concentrations in French fries to range from lower than 60 to $1220 \mu \mathrm{g} / \mathrm{kg}$ and in potato crisps from 310 to 2800 $\mu \mathrm{g} / \mathrm{kg}$. This means that the potential for misclassification of exposure when assigning a single mean acrylamide concentration value to individual foods is considerable.

To investigate whether using mean acrylamide concentrations in foods (the same that were applied in the Netherlands Cohort Study on diet and cancer) results in a sound estimate of total acrylamide intake, we did a validation study using Dutch 24-hour duplicate diets. 


\section{Materials and methods}

\section{4-hour duplicate diets}

The National Institute for Public Health and the Environment (RIVM) in the Netherlands has collected 24-hour duplicate diets of 123 persons in the spring and autumn of 2004. The participants represent the 18-74-year-old male and female segment of the Dutch population and were a cross-section of all social classes. Information on gender, age and body weight of the participants was collected. The design and sampling procedures were the same as applied in an earlier duplicate diet study that was done in 1994 by the RIVM and described by Sizoo et al. (18). 24-hour duplicate diets were collected during the spring and autumn to take seasonal fluctuations in food composition and consumption into account. All participants collected a duplicate portion of everything they consumed (foods, and drinks, including drinking water) in the 24-hour sampling period in a stainless steel bucket, which was placed in a styropor box, containing dry ice and cooling elements. They were asked to provide information about the quantity of the various foods and drinks consumed in practical units (e.g. tablespoons, cups, pieces, glasses) rather than in exact amounts, such as grammes or millilitres. After collection, the diets were freeze-dried and homogenised. During the process of freeze-drying, the samples were exposed to room temperature for a few days and were thereafter stored at $-20^{\circ} \mathrm{C}$. Subsequently, the dry weight of the freeze-dried diets was determined.

\section{Acrylamide intake estimation}

For all 123 participants of the 2004 duplicate diet study, the theoretical acrylamide intake on the day of participation was calculated, based on a list that participants completed about the portions of each consumed food and drink. The mean acrylamide concentrations assigned to each food and drink were based on analytical data of foods on the Dutch market, which were analysed by the Food and Consumer Product Safety Authority (VWA) in 2002 and 2005, as described elsewhere $(11,17)$. In 2002, various foods, such as bread, French fries, pastry and cake, Dutch spiced cake, crisps, corn flakes, meat, fish, and several beverages were sampled and analysed for acrylamide following an elaborated sampling scheme. In 2005, an additional series of foods was analysed to specifically accommodate the estimation of acrylamide intake of the NLCS cohort and this series consisted of rusk, several types of biscuits, rye bread, crisp bread, pastry and cake, chocolate and chocolate milk, nuts and salty snacks, peanut butter, and coffee. In addition, several types of bread were analysed again in 2005 because the quantitation limit decreased from 30 to 15 $\mu \mathrm{g} / \mathrm{kg}$, which is relevant for this type of product, because it contains acrylamide concentrations below the quantitation limit. Acrylamide measurements in cookies were done in several types known to be eaten most frequently by a population comparable to the NLCS population. Thus, the acrylamide level for cookies was based on the acrylamide level of the specific types of cookies weighted by the frequency of consumption of each type in the NLCS-comparable population.

In order to have $80 \%$ statistical power (with $\alpha=0.05$ ) to detect a correlation of 0.4 or higher between the estimated and measured acrylamide content of 24-hour duplicate 
diets, 39 diets had to be analysed for acrylamide. These 39 diets were selected from the 123 diets on the basis of a range of theoretical acrylamide concentrations and inclusion of the main acrylamide-contributing foods to the daily dietary acrylamide intake in the Netherlands. These include Dutch spiced cake, coffee, French fries, potato crisps, and dough products. In addition, a few theoretically low acrylamide containing diets were selected.

\section{Acrylamide determination}

The method of acrylamide analysis was according to that developed by Rosén and Hellenäs (19-21), which is classified as a Nordic Committee on Food Analysis (NMKL) method (22). In short, after homogenisation and addition of deuterium-labeled acrylamide, the samples were extracted with water. After centrifugation of the extract, an aliquot of the supernatant was subjected to solid-phase extraction and subsequently injected into an LCMS-MS system.

\section{Statistical analysis}

Spearman correlation coefficients were calculated with SPSS software (package 15). Student's t-tests were done to compare acrylamide intake between men and women and spring and autumn respectively.

ANOVA was used to compare the mean acrylamide intake of different age categories. Two-sided $\mathrm{P}$ values are reported throughout this paper.

\section{Results}

The mean age of the 61 participants in the spring was 43 y (range 18-69 y), whereas the mean age of the 62 participants in the autumn was 44 y (range: 18-74 y). For 122 24-hour duplicate diets, the acrylamide intake could be estimated. For one diet, the food item list was not complete. On average, the estimated acrylamide intake based on the 122 diets was $0.45 \mu \mathrm{g} / \mathrm{kg}$ BW (SD: $0.49 \mu \mathrm{g} / \mathrm{kg}$ BW; range: $0-3.77 \mu \mathrm{g} / \mathrm{kg} \mathrm{BW} ; 20^{\text {th }}, 40^{\text {th }}, 60^{\text {th }}$ and $80^{\text {th }}$ percentiles: $0.12,0.22,0.42,0.68 \mu \mathrm{g} / \mathrm{kg} \mathrm{BW})$.

The most important foods contributing to the mean acrylamide intake through the 122 diets are plotted in Figure 1 . They comprised potato crisps (25\%), coffee (18\%), Dutch spiced cake $(10 \%)$, fried potatoes $(10 \%)$, and French fries $(7 \%)$. The acrylamide intake for men and women was not statistically significantly different ( $P>0.05)$.

The mean acrylamide intake for the participants in spring was $0.57 \mu \mathrm{g} / \mathrm{kg} \mathrm{BW}$ and significantly higher $(P<0.01)$ than the mean acrylamide intake for the participants in autumn, which was $0.33 \mu \mathrm{g} / \mathrm{kg}$ BW. For participants in the age categories <30y (24 participants), 3040y (29 participants), 40-50y (30 participants), 50-60y (20 participants) and $>60 \mathrm{y}$ (19 participants) the mean dietary acrylamide intake was $0.39,0.53,0.58,0.35$, and 0.34 $\mu \mathrm{g} / \mathrm{kg} \mathrm{BW}$, respectively, and not statistically significantly different between the groups. 


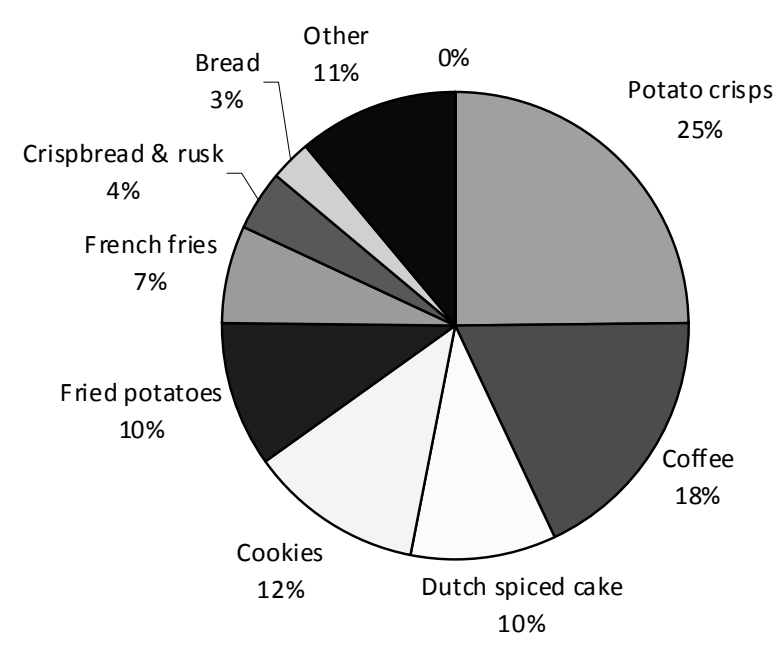

Figure 1: Contribution of the most important food groups to the dietary exposure of acrylamide by 24-hour duplicate diets $(\mathrm{N}=122)$

The 39 diets selected for chemical analysis inferred a mean theoretical acrylamide intake of $0.70 \mu \mathrm{g} / \mathrm{kg}$ BW (SD: $0.65 \mu \mathrm{g} / \mathrm{kg}$ BW; range: $0.05-3.77 \mu \mathrm{g} / \mathrm{kg} \mathrm{BW} ; 20^{\text {th }}, 40^{\text {th }}, 60^{\text {th }}$ and $80^{\text {th }}$ percentiles: $\left.0.20,0.49,0.70,1.03 \mu \mathrm{g} / \mathrm{kg} \mathrm{BW}\right)$. The most important foods contributing to the mean acrylamide content of the 39 diets selected for analysis were potato crisps (34\%), Dutch spiced cake (16\%), French fries (11\%), and coffee (13\%). The mean acrylamide intake of the 39 meals based on chemical analysis was $0.80 \mathrm{ug} / \mathrm{kg}$ BW (SD: 1.43 ug/kg BW; range: 0.03-6.53 ug/kg BW; 20th, 40th, 60th and 80th percentiles: 0.13, 0.30, $0.50,0.75 \mathrm{ug} / \mathrm{kg} \mathrm{BW})$.

In Figure 2, the estimated acrylamide content of the 39 diets is plotted against the measured acrylamide content in these diets. The Spearman correlation coefficient between the estimated and the measured acrylamide contents was 0.82 with a $p$-value of $<0.001$. For two of the 39 duplicate diets the measured acrylamide content was more than five times higher than the estimated acrylamide content.

\section{Discussion}

The results of this study showed a good correlation between the measured acrylamide content and the estimated acrylamide content of 39 24-hour duplicate diets. This shows that assigning a mean acrylamide value to individual foods, which was done to estimate 
the acrylamide content of the duplicate diets, resulted in a good rank estimate of the true acrylamide content.

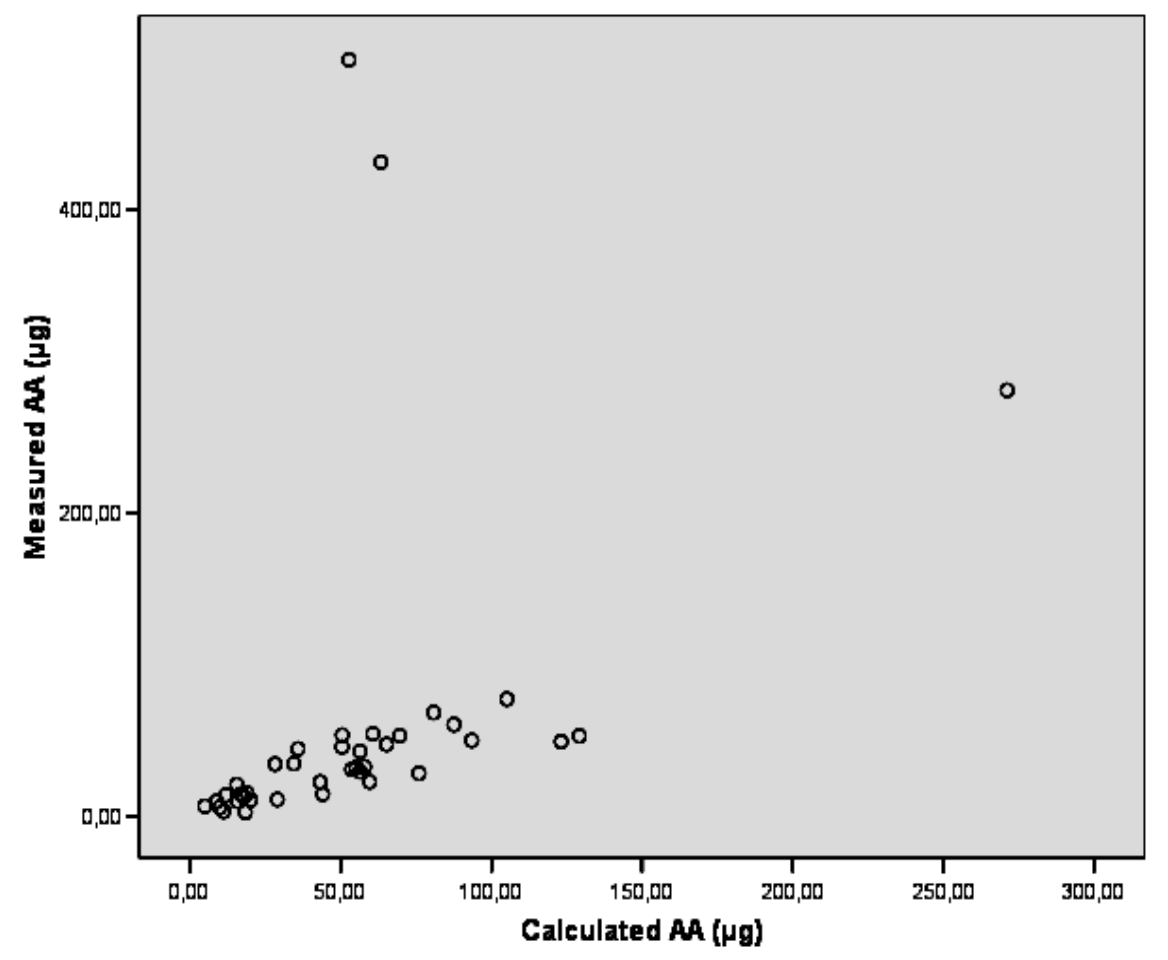

Figure 2: Correlation between measured and estimated acrylamide (AA) content of 39 24-hour duplicate diets

The two participants with outlying measured acrylamide content compared with the estimated acrylamide content reported to have consumed French fries in one case and olives in the other case. For French fries, the mean level of acrylamide was based on ready-to-eat products bought in snack bars and on ready-to-eat fries prepared from frozen fries bought in supermarkets and prepared in the laboratory according to instructions on the package. The participant who consumed French fries might have prepared the fries at home and preferred them browner than they would have been after following normal preparation instructions, resulting in a higher acrylamide level. Furthermore, the mean acrylamide level of green and black olives was used for the estimated acrylamide content of the duplicate diets. Black olives contain higher concentrations of acrylamide than green olives. For the participant who reported eating olives in the duplicate diet study it was unknown whether green or black olives were consumed.

At a European Food Safety Authority (EFSA) scientific colloquium on acrylamide carcinogenicity in 2008 (23), dietary exposure data across Europe were discussed. It was generally concluded that it was not the chemical analysis contributing to the uncertainty in dietary acrylamide exposure assessment, but rather consumption data and the use of single mean acrylamide concentrations for individual foods. The Food and Consumer 
Product Safety Authority laboratory that analysed the Dutch foods for this study participated in a number of proficiency programs of the Food Analysis Performance Assessment Scheme (FAPAS) and the Institute for Reference Materials and Measurements (IRMM) from 2002-2008, which resulted in satisfactory z-scores between -2 and 2 for acrylamide in the matrices crisp bread, potato crisps, coffee, breakfast cereal and bread, meaning that the analytical data were acceptable. Participation in a collaborative study for the determination of acrylamide in roasted coffee (24) led to satisfactory results also.

This duplicate diet study addressed the question whether single mean acrylamide concentrations for individual foods can be applied, because the range in concentrations per food can be large, as mentioned earlier. An important asset of the mean acrylamide concentrations used in this study is that they are representative for the population in the study, because we used acrylamide concentrations in foods from the Dutch market only.

Sampling was based on a sampling plan in which the following factors were taken into account: representativeness of the market, regional variations, and for all foods, most brands and varieties, including different production codes or seasonal variations, were sampled and analysed (17). For this study, we used acrylamide concentrations in foods that were sampled and analysed in 2002 and 2005. It has been shown that after 2005, acrylamide concentrations in some products have decreased compared with acrylamide concentrations as quantified in $\mathbf{2 0 0 2}$ for these products. Since the discovery of acrylamide in foods, industry together with science are working on mitigation procedures and managed to reduce acrylamide concentrations, e.g. in Dutch spiced cake and potato crisps (2527). Thus, care has to be taken in the assignment of acrylamide concentrations to individual foods for acrylamide intake assessments in the future

The fact that there was a good correlation between the estimated and the measured acrylamide content of the duplicate diets also implies that the main foods and drinks contributing to acrylamide intake are included in the intake assessment. For the Dutch population, it can be concluded that fried potato products, coffee, cookies and Dutch spiced cake are the main contributors to acrylamide intake.

As far as we know, there has not been any study that can be directly compared with the present duplicate diet study. However, there have been a few studies that have correlated FFQ acrylamide data to a biomarker for acrylamide exposure, namely acrylamide (and glycidamide) to haemoglobin adducts (28-32). This enables investigating both whether using mean acrylamide concentrations for individual foods is reliable, and wether the performance of the FFQ with regard to estimating the long-term intake of acrylamidecontributing foods is adequate. However, one has to realise that FFQs and haemoglobin adducts cannot be expected to correlate perfectly, because they measure different aspects of acrylamide exposure. Most FFQs aim to estimate intake of acrylamide over a considerable period, often the previous year, whereas haemoglobin adducts account for exposure, absorption and metabolism of acrylamide over the previous four months. Furthermore, the biomarker is not specific for dietary acrylamide intake, but also represents acrylamide exposure from other sources, e.g. smoking.

Kütting et al. found no correlation between the estimated daily acrylamide intake and acrylamide haemoglobin adducts, investigated among only 9 non-smoking pregnant 
women (28). However, the authors had not used acrylamide data for individual foods for the intake estimation (only frequencies and portions of foods), because they were of the opinion that using those individual concentrations would not improve the assessment, due to the large variation of acrylamide concentrations within foods. Their main conclusion was that the missing correlation was due to a restricted validity of anamnestic dietary data. However, they do not show a scatter plot for estimated dietary acrylamide intake versus haemoglobin adducts, which makes it impossible to judge whether outliers may have diluted a possible correlation.

In 2008, Kütting et al. published results of a larger study in 1008 German volunteers (29). A statistically significant, but weak, correlation of 0.18 was found in non-smoking women, and 0.17 in non-smoking men. Median dietary acrylamide concentrations in foods as reported by the German Federal Institute of Risk Assessment (BfR) were used. The authors indicate that their study population was probably very health-conscious due to the method they used to select the population. Thus, it is questionable whether acrylamide data from the BfR are representative of the foods consumed by this population.

A study by Bjellaas et al. found no correlation between acrylamide haemoglobinadduct levels and total dietary intake of acrylamide as calculated via an FFQ (30), only between acrylamide haemoglobin adducts levels and the reported individual crisp/snack and cracker consumption. However, in this study, several (foreign) databases of acrylamide concentrations in foods were used, and thus again it can be questioned whether the acrylamide concentrations used were representative for products consumed by the population in the study. The authors indicate that the quality of the FFQ, the large variation in acrylamide contents within foods, and the difficulty to estimate the contribution to acrylamide intake from home-cooked foods might be the reasons for the absence of a correlation.

Wirfält et al. reported lack of a significant correlation between dietary acrylamide estimates and haemoglobin acrylamide adducts in Swedish non-smoking women (31), whereas moderate positive correlations were found for smoking men ( $r=0.37)$ and women $(r=0.59)$, and for non-smoking men $(r=0.60)$. The authors used acrylamide data from a national database only. The reason for the absence of a moderate correlation in nonsmoking women remains unclear. The authors mentioned the limited sample size of the study, the fact that dietary data were not collected with the aim to study acrylamide intakes, and differences in bioavailability. Apart from the limited sample size, these do not seem to be explanations for the absence of a correlation in non-smoking women specifically. However, the range of estimated acrylamide intake was lower in the subgroup of non-smoking women than in the other subgroups and the contribution of different foods to the total intake differed also from the other subgroups. Furthermore, this particular subgroup of non-smoking women may have been more exposed to environmental tobacco smoke that also contains acrylamide, which then blurs the association between dietary acrylamide intake and acrylamide to haemoglobin adducts.

Wilson et al. (32) observed a correlation of 0.34 between FFQ-estimated dietary acrylamide intake and the sum of acrylamide and glycidamide adducts in 296 non-smoking women. They used acrylamide data from a national database only. To calculate the sum of 
these adducts is a better approach than to measure acrylamide adducts alone, because both adducts originate from acrylamide intake.

What can be concluded from these studies correlating biomarkers and FFQ data is that correlations tend to be higher when data of acrylamide in foods from the national market are used and possibly also when the sum of acrylamide and glycidamide to haemoglobin adducts is used to correlate to the FFQ data. Other possible reasons why the observed correlations vary are differences in the quality of the FFQ that was used, and the foods that contribute most to the dietary acrylamide intake in the specific study populations. Some foods have a larger variation of acrylamide concentrations than other foods. Of course, these foods would have to be included in the FFQ as separate items.

This duplicate diet study did not address the question whether respondents to an FFQ are able to accurately report on their average intake of acrylamide-containing foods (usually during a year) and whether a single measurement of food intake through an FFQ characterizes chronic average dietary acrylamide intake. Both are required to study the association between acrylamide intake and cancer risk when using FFQs. These two aspects have to be investigated also to be able to judge the validity and reliability of the estimation of dietary acrylamide intake through FFQs. This can be done for instance by comparing the FFQ data to dietary records to investigate the validity of the FFQ, and by repeatedly administering the FFQ to study the stability of the dietary habits over time, both of which were done in for instance the NLCS study $(33,34)$. Nevertheless, this duplicate diet study and the studies on the biomarkers mentioned above rendered some reassuring indications that an important part of the acrylamide intake assessment through questionnaires, namely assigning single values to individual foods, is not necessarily as fruitless as thought by some $(28,35)$.

In conclusion, we have shown that using mean acrylamide values for individual foods does not preclude dietary acrylamide intake assessment through questionnaires, despite the large variation of acrylamide concentrations within single foods. However, there may be some boundary conditions, such as that acrylamide values from national databases are used, and that the main acrylamide-contributing foods in the study population are the foods with less variation in acrylamide concentrations. Furthermore, the observed correlation strengthens the confidence in the validity of the acrylamide intake assessment in the NLCS, and thus also in the validity of the within the NLCS observed associations between dietary acrylamide intake and risk of several cancer types. 


\section{References}

1. Tareke E, Rydberg P, Karlsson P, Eriksson S, Tornqvist M. Analysis of acrylamide, a carcinogen formed in heated foodstuffs. J Agric Food Chem 2002;50:4998-5006.

2. IARC. Monographs on the evaluation of carcinogen risk to humans: some industrial chemicals. Lyon: International Agency for Research on Cancer, 1994.

3. Summary and conclusions of the sixty-fourth meeting of the Joint FAO/WHO Expert Committee on Food Additives (JECFA). JECFA, 2005.

4. Mottram DS, Wedzicha BL, Dodson AT. Acrylamide is formed in the Maillard reaction. Nature 2002;419:4489.

5. Stadler RH, Blank I, Varga N, et al. Acrylamide from Maillard reaction products. Nature 2002;419: 449-50.

6. Pelucchi C, Galeone C, Levi F, et al. Dietary acrylamide and human cancer. Int J Cancer 2006;118:467-71.

7. Mucci LA, Dickman PW, Steineck G, Adami HO, Augustsson K. Dietary acrylamide and cancer of the large bowel, kidney, and bladder: absence of an association in a population-based study in Sweden. Br J Cancer 2003;88:84-9.

8. Mucci LA, Lindblad P, Steineck G, Adami HO. Dietary acrylamide and risk of renal cell cancer. Int J Cancer 2004;109:774-6.

9. Mucci LA, Adami HO, Wolk A. Prospective study of dietary acrylamide and risk of colorectal cancer among women. Int J Cancer 2006;118:169-73.

10. Mucci LA, Sandin S, Balter K, Adami HO, Magnusson C, Weiderpass E. Acrylamide intake and breast cancer risk in Swedish women. Jama 2005;293:1326-7.

11. Hogervorst JG, Schouten LJ, Konings EJ, Goldbohm RA, van den Brandt PA. A Prospective Study of Dietary Acrylamide Intake and the Risk of Endometrial, Ovarian and Breast Cancer. Cancer Epidemiol Biomarkers Prev 2007:16(11):2304-13.

12. Hogervorst JG, Schouten LJ, Konings EJ, Goldbohm RA, van den Brandt PA, Dietary acrylamide intake and the risk of renal cell, bladder, and prostate cancer. Am J Clin Nutr 2008;87(5):1428-38.

13. Thonning Olsen $\mathrm{P}$, Olsen A, Frandsen H, Frederiksen K, Overad K, Tjonneland A. Acrylamide exposure and incidence of breast cancer among postmenopausal women in the Danish Diet, Cancer and Health Study. In J Cancer 2008;122(9):2094-2100.

14. Larsson SC, Håkansson N, Akesson A, Wolk A. Long-term dietary acrylamide intake and risk of endometrial cancer in a prospective cohort of Swedish women. Int J Cancer 2009;124(5):1196-9.

15. Larsson SC, Akesson A, Wolk A. Long-term dietary acrylamide intake and breast cancer risk in a prospective cohort of Swedish women. Am J Epidemiol 2009;169(3), 376-81.

16. Konings EJ, Hogervorst JGF, Schouten LJ, van den Brandt PA. Assessing exposure levels of acrylamide. In: Skog K, Alexander J, eds. Acrylamide and other hazardous compounds in heat-treated foods. Cambridge: Woodhead Publishing Limited, 2006:214-225.

17. Konings EJ, Baars AJ, van Klaveren JD, et al. Acrylamide exposure from foods of the Dutch population and an assessment of the consequent risks. Food Chem Toxicol 2003;41:1569-79.

18. Sizoo EA, van Egmond HP. Analysis of duplicate 24-hour diet samples for aflatoxin $B_{1}$, aflatoxin $M_{1}$ and ochratoxin A. Food Add and Contam. 2005;22(2):163-72.

19. Rosén J, Hellenäs K-E, Analysis of acrylamide in cooked foods by liquid chromatography tandem mass spectrometry. The Analyst 127, 880-882.

20. Tareke E, Rydberg P, Karlsson, Eriksson S, Törnqvist, "Analysis of Acrylamide, a carcinogen formed in heated foodstuffs. J Agric Food Chem, 2002, 50:4998-5006.

21. Fohgelberg P, Rosen J, Hellenas KE, Abramsson-Zetterberg L. The acrylamide intake via some common baby food for children in Sweden during their first year of life - an improved method for analyses of acrylamide. Food Chem Toxicol, 2005, 43 (6):951-9.

22. Nordic Committee on Food Analysis (NMKL) method No. 185. Acrylamide: Determination in bakery and potato products by liquid chromatography tandem mass spectrometry (LC-MS-MS).

23. Summary Report EFSA scientific colloquium no. 11. Acrylamide carcinogenicity new evidence in relation to dietary exposure. 22-23 May 2008, Tabiano (PR), Italy.

24. Wenzl T, Szilagyi S, Rosen J, Karasek L. Validation of an analytical method to determine the content of acrylamide in roasted coffee. JRC, IRMM report EUR 23403 EN, 2008.

25. Konings EJ, Ashby P, Hamlet, CG, Thompson GA. Acrylamide in cereal and cereal products: a review on progress in level reduction. Food Addit Contam 2007:24 Suppl 1, 47-59.

26. Anonymous. Acrylamide levels in foods on the Dutch Market. Food and Consumer Products Safety Authority (VWA). Report, April 2007 (in Dutch). 
27. Acrylamide "Toolbox": the European food and drink industry (CIAA). Bruxelles: CIAA, 2007.

28. Kütting B, Schettgen T, Beckman MW, Angerer J, Drexler H. Influence of Diet on Exposure to acylamide Reflection on the validity of a Questionnaire. Ann Nutr Metab 2005;49(3):173-7.

29. Kütting B, Uter W, Drexier H. The association between self-reported acrylamide intake and hemoglobin adducts as biomarkers of exposure. Cancer Causes Control 2008;19:273-81.

30. Bjellaas T, Olesen PT, Frandsen H, Haugen, M, Stølen LH, Paulsen JE, Alexander J, Lundanes E, Becher G. Comparison of estimated dietary intake of acrylamide with hemoglobin adducts of acrylamide and glycidamide. Toxicological sciences 2007, 98 (1):110-7.

31. Wirfält E, Paulsson B, Törnqvist M, Axmon A, Hagmar L. Associations between estimated acrylamide intakes, and hemoglobin AA adducts in a sample from the Malmö diet and cancer cohort. Eur J Clin Nutr 2008;62:314-23.

32. Wilson KM, Vesper HW, Tocco P, Sampson L, Rosén J, Hellenäs K-E, Törnqvist M, Willett WC. Validation of a food frequency questionnaire measurement of dietary acrylamide intake using hemoglobin adducts of acrylamide and glycidamide. Cancer Causes Control 2009;20(3)8:269-78.

33. Goldbohm RA, van den Brandt PA, Brants HA, et al. Validation of a dietary questionnaire used in a largescale prospective cohort study on diet and cancer. Eur J Clin Nutr 1994;48:253-65.

34. Goldbohm RA, van 't Veer, van den Brandt PA, van 't Hof A, Brants AM, Sturmans F, Hermus RJJ. Reproducibility of a food frequency questionnaire and stability of dietary habits determined from five annually repeated measurements. Eur J Clin Nutr, 1995;49(6):420-9.

35 Doerge DR, Young JF, Chen JJ, DiNovi MJ, Henry SH. Using dietary exposure and physiologically based pharmacokinetic/pharmacodynamic modeling in human risk extrapolations for acrylamide toxicity. J Agric Food Chem 2008;56: 6031-6038. 


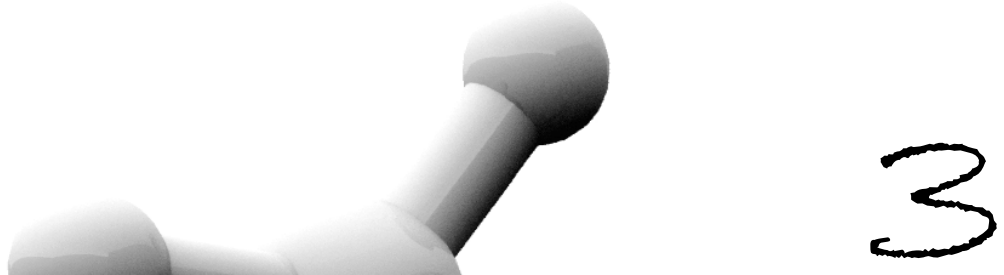

\section{A prospective study of dietary acrylamide intake and the risk of endometrial, ovarian, and breast}

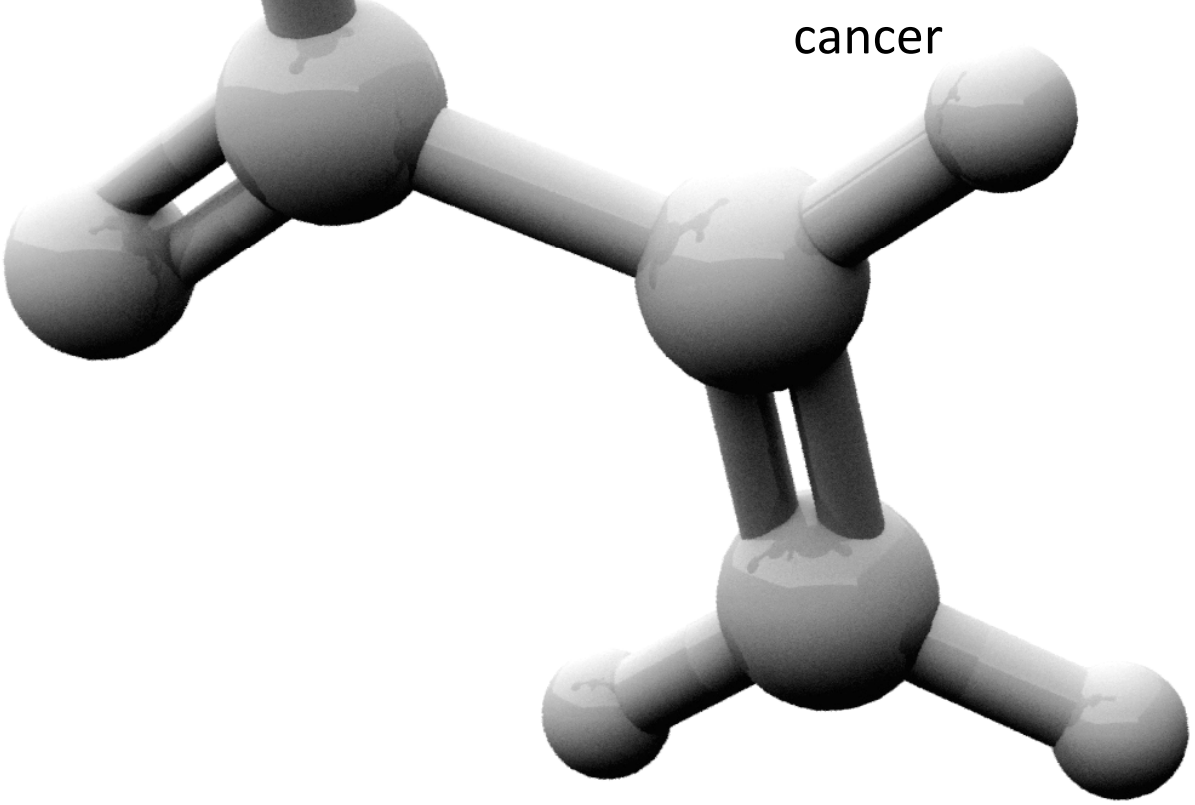

Janneke G.F. Hogervorst

Leo J. Schouten

Erik J.M. Konings

R. Alexandra Goldbohm

Piet A. van den Brandt 


\section{Abstract}

Background Acrylamide, a probable human carcinogen, was detected in various heattreated carbohydrate-rich foods in 2002. The few epidemiological studies done thusfar have not shown a relationship with cancer. Our aim was to investigate the association between acrylamide intake and endometrial, ovarian, and breast cancer risk.

Methods The Netherlands Cohort Study on diet and cancer includes 62,573 women, aged 55-69 years. At baseline (1986), a random subcohort of 2,589 women was selected using a case-cohort approach for analysis. The acrylamide intake of subcohort members and cases was assessed with a food frequency questionnaire and was based on chemical analysis of all relevant Dutch foods. Subgroup analyses were done for never-smokers to eliminate the influence of smoking; an important source of acrylamide.

Results After 11.3 years of follow-up, 327, 300, and 1,835 cases of endometrial, ovarian, and breast cancer, respectively, were documented. Compared with the lowest quintile of acrylamide intake (mean intake, $8.9 \mu \mathrm{g} / \mathrm{day}$ ), multivariable-adjusted hazard ratios (HR) for endometrial, ovarian, and breast cancer in the highest quintile (mean intake, $40.2 \mu \mathrm{g} /$ day) were 1.29 (95\% Cl 0.81-2.07; P-trend 0.18), 1.78 (95\% Cl 1.10-2.88; P-trend 0.02), and 0.93 (95\% Cl 0.73-1.19; P-trend 0.79), respectively. For never-smokers, the corresponding HRs were: 1.99 (95\% Cl 1.12-3.52; P-trend 0.03), 2.22 (95\% Cl 1.20-4.08; P-trend 0.01), and 1.10 (95\% Cl 0.80-1.52; P-trend 0.55).

Conclusions We observed increased risks of postmenopausal endometrial and ovarian cancer with increasing dietary acrylamide intake, particularly among never-smokers. Risk of breast cancer was not associated with acrylamide intake. 


\section{Introduction}

In 2002, Swedish researchers reported the presence of acrylamide, classified by the IARC as a probable human carcinogen, in several heat-treated carbohydrate-rich foods (1). This message received a lot of media attention and caused concern among the general population and the scientific community, particularly because acrylamide is present in foods at considerably higher concentrations than other well-known food carcinogens, such as polycyclic aromatic hydrocarbons and ethyl carbamate (2). Before this date, acrylamide exposure was thought to occur mainly through occupational exposure, because it has been widely used in industry since the 1950s, and further through cigarette smoke and, to a minor extent, through drinking water $(3,4)$. Since 2002 , research in several countries has also shown high acrylamide concentrations in foods such as French fries, coffee, potato crisps, and cookies (4). In the Netherlands, high concentrations of acrylamide were found in the typically Dutch spiced cakes (5), which are often consumed daily.

Acrylamide has been shown to be mainly formed in food by Maillard browning reactions in which amino acids, mainly asparagine, react with reducing sugars at high temperatures $\left(>120^{\circ} \mathrm{C}\right)(6-8)$.

It is generally thought that acrylamide might cause cancer through cytochrome P4502E1 (CYP2E1)-catalysed conversion to glycidamide, which has proven to be genotoxic (4).

There is a lot of debate on whether acrylamide in food justifies the concern that it provoked in 2002. Animal studies have shown positive dose-response relationships between acrylamide administered in drinking water and cancer in multiple organs in both mice and rats (9-12), among which were several hormone-sensitive organs, such as the mammary glands and the uterus. However, studies on humans with occupational acrylamide exposure have been negative thus far, apart from an increased risk of pancreatic cancer, but this was based on a small number of cases (13-18). Dietary acrylamide intake and its relation with various types of cancer has been studied in few case-control studies (19-21) and only two prospective cohort studies on breast and colorectal cancer $(22,23)$. None of these has found indications for a positive relationship.

Because of the pitfalls of case-control studies and the paucity of cohort studies, there is a need of more prospective cohort studies. In the present study, we investigated the relationship between dietary acrylamide and cancer of the endometrium, ovaries and breasts within the framework of the Netherlands Cohort Study on diet and cancer (NLCS). These cancer types were chosen because of their association with acrylamide in animal studies, and because there are indications of a positive association between fried food consumption and both ovarian and breast cancer $(24,25)$. We did subgroup analyses for women who reported to have never smoked in order to eliminate the influence of smoking, which is a major source of acrylamide exposure. 


\section{Subjects and methods}

\section{Study cohort, cases and follow-up}

The Netherlands Cohort Study on diet and cancer started in September 1986 with the enrolment of 62,573 women aged 55 to 69 years. All of these women were presumed to be post-menopausal. At baseline, data on dietary habits and other risk factors were collected by means of a self-administered questionnaire. The informed consent for this study consisted of the completing and returning of the questionnaire in 1986. Participants were informed that by returning a completed questionnaire they gave consent to participate in a study about the etiology of cancer and for record linkage to the Netherlands Cancer Registry and the Netherlands Pathology Registry. This procedure of informed consent was approved by the Medical Ethics Committees of the University Hospital Maastricht and TNO Nutrition in February 1985 and July 1986, respectively.

Data processing and analysis were based on the case-cohort approach because the desired level of detail of the questionnaire (e.g., including open-ended questions) prohibited high-throughput processing. Therefore, the questionnaires were not processed for the total cohort, but only for a subcohort and the cases. Following the case-cohort approach, cases were enumerated for the entire cohort (providing the numerator information for estimating incidence rates), whereas the accumulated person-years for the entire cohort were estimated from a subcohort of 2,589 women randomly sampled from the entire cohort at baseline (providing the denominator information for estimating incidence rates). Thus, all the incident cases were used in the analyses. The size of the subcohort was based on calculations that were described in a previous publication (26). Since the start of the study, the subcohort has been followed up regularly for vital status information. Incident cases in the total cohort have been detected by annual computerised record linkages to the regional cancer registries and the Netherlands Pathology Registry. The completeness of cancer follow-up through linkage with the national cancer registry was estimated to be at least $96 \%$ (27). The follow-up of the subcohort was $100 \%$ complete at the end of the follow-up period. Further details on the design of the study and methods of follow-up are presented elsewhere (26, 28-30).

The analyses are based on 11.3 years of follow-up, from September 1986 through December 1997. This is the most recent dataset available for analysis because cancer registry procedures, linkage to the cancer registry, checks of the reported linkages and the processing of the food-frequency questionnaire cause considerable delays. After 11.3 years of follow-up, there were 327,300 , and 1,835 incident, microscopically confirmed, primary carcinomas of the endometrium ([ICD-O]-3:C54), ovaries ([ICD-O]-3:C56.9) and breasts ([ICD-O]-3:C50), respectively. Cases were excluded from analysis if they had been diagnosed with non-epithelial tumours and borderline invasive tumours, and if their dietary data were incomplete or inconsistent. Subcohort members were excluded from the analysis if they had been diagnosed with cancer other than skin cancer at baseline, if information on dietary habits was missing or inconsistent and if they reported at baseline to have had a hysterectomy (excluded from the endometrial cancer analysis) or an oophorectomy (excluded from the ovarian cancer analysis). 
Figure 1 shows the selection and exclusion steps that resulted in the numbers of cases and subcohort members that were available for analysis.

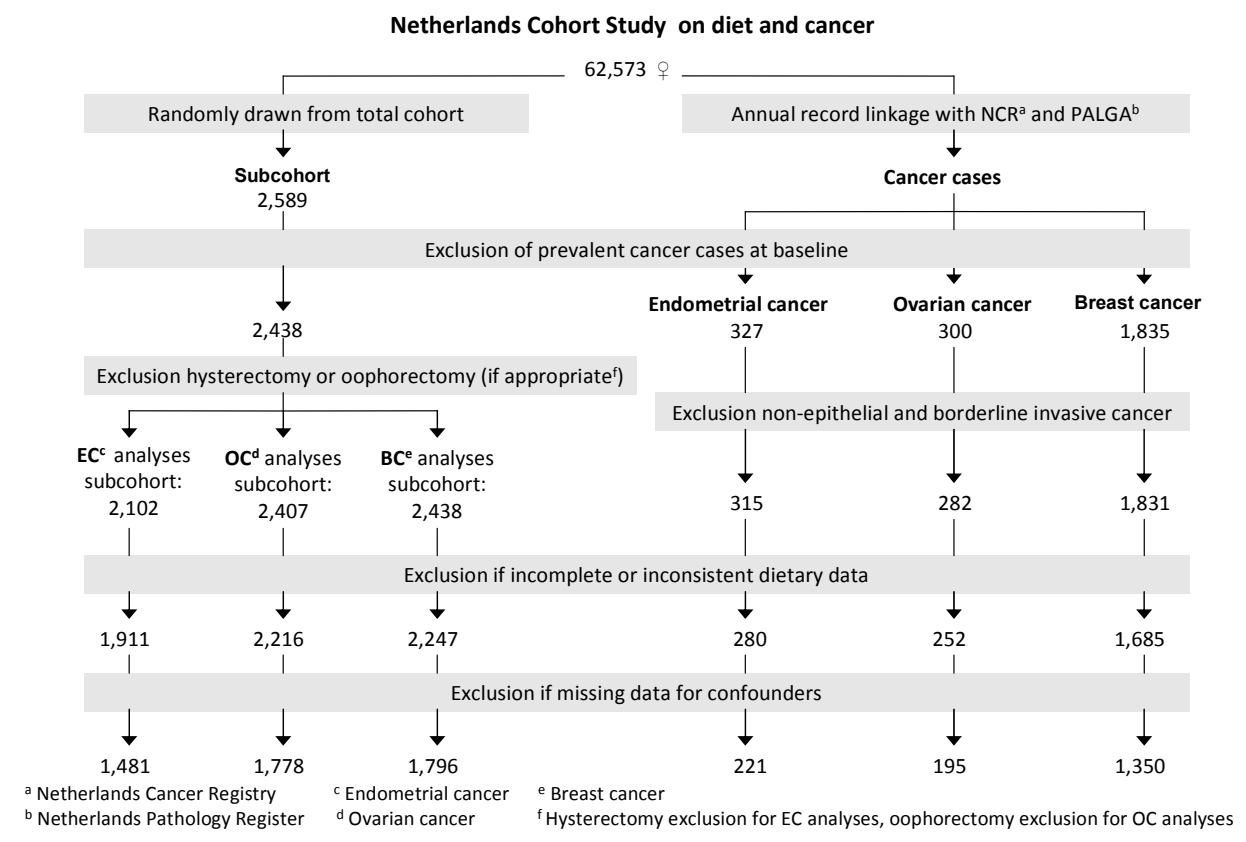

Figure 1: Flow diagram of subcohort members and cases on whom the analyses were based

\section{Acrylamide intake assessment}

In the NLCS, a food frequency questionnaire with questions on 150 food items was used to estimate dietary habits (29). The acrylamide intake was estimated from the mean acrylamide level of food items, and the frequency of consumption and portion size of the food items.

For the acrylamide intake estimation in the NLCS, we focused on foods that are likely to contain considerable acrylamide concentrations, according to the mechanism of formation and reports from various European countries (31). Thus, the following list of food products was established: French fries, potato crisps, bread and rye bread, cookies, pastry, rusk, crisp bread, chocolate and chocolate milk, nuts, peanut butter, breakfast cereals, and coffee. In order to obtain an intake estimate representative for the Dutch situation, we used data on acrylamide concentrations in food products on the Dutch market.

In 2002, the Dutch Food and Consumer Product Safety Authority analysed acrylamide concentrations in various foods, such as bread, French fries, pastry and cake, Dutch spiced cake, crisps, cornflakes, meat, fish, and several beverages, following an elaborate sampling scheme (5). In 2005, another series of foods was analysed to specifically accommodate the estimation of acrylamide intake of the NLCS cohort and this series consisted of bread, rusk, specific types of biscuits, rye bread, crisp bread, pastry and cake, chocolate and 
chocolate milk, nuts and salty snacks, peanut butter, and coffee (31). Several types of bread were analysed again in 2005, because the quantitation limit of the analytical method had decreased from $30 \mathrm{ppb}$ (even $60 \mathrm{ppb}$ for some foods) in 2002 to $15 \mathrm{ppb}$ in 2005 , which offered the opportunity to more accurately estimate the acrylamide content of bread. Acrylamide in coffee was analysed in brewed filtered coffee that was prepared according to general Dutch preparation instructions $(7 \mathrm{~g}$ of coffee powder/125 $\mathrm{ml}$ of water). Acrylamide measurements in cookies were done in several types of cookies known to be eaten most frequently by a population comparable to the NLCS, according to information from the development phase of the questionnaire. Thus, the acrylamide level for cookies was based on the acrylamide level of the specific types of cookies weighted by the frequency of consumption of the NLCS-comparable population. The same was done for other food items, such as pies and chocolates.

The foods listed above were assigned the mean value of the acrylamide values per food or a value half the quantitation limit when concentrations were lower than the quantitation limit, because they probably do contain some acrylamide. Other foods (e.g., meats, cooked and raw vegetables, dairy products) were assigned the value zero. Table 1 shows the acrylamide concentrations of foods that were used in the acrylamide intake assessment.

Table 1. Acrylamide concentrations in foods, used for the NLCS acrylamide intake assessment

\begin{tabular}{lcccc}
\hline & n samples & $\begin{array}{c}\text { mean } \\
(\mu \mathrm{g} / \mathrm{kg})\end{array}$ & $\begin{array}{c}\text { minimum } \\
(\mu \mathrm{g} / \mathrm{kg})\end{array}$ & $\begin{array}{c}\text { maximum } \\
(\mu \mathrm{g} / \mathrm{kg})\end{array}$ \\
\hline Potato crisps & 40 & 1249 & 310 & 2800 \\
Dutch spiced cake & 13 & 1018 & 260 & 1410 \\
French fries & 33 & 351 & $<\mathrm{LOQ}^{*}$ & 1220 \\
Salty snacks & 12 & 277 & 45 & 867 \\
Crisp bread & 12 & 229 & 15 & 914 \\
Cookies & 20 & 204 & 10 & 829 \\
Cornflakes & 12 & 121 & $<\mathrm{LOQ}^{*}$ & 300 \\
Peanut butter & 2 & 107 & 118 \\
Chocolate & 6 & 60 & 22 & 116 \\
Nuts & 8 & 33 & $<\mathrm{LOQ}^{\dagger}$ & 83 \\
Rusk & 3 & 25 & 16 & 33 \\
Rye bread & 13 & 24 & $<\mathrm{LOQ}^{\dagger}$ & 60 \\
Sweet pastry & 19 & 18 & $\mathrm{LOQ}^{\dagger}$ & 111 \\
Coffee & 9 & 17 & $<\mathrm{LOQ}^{\dagger}$ & 28 \\
Chocolate milk & 4 & $<\mathrm{LOQ}^{\dagger}$ & $<\mathrm{LOQ}^{\dagger}$ \\
Bread & 22 & $<\mathrm{LOQ}^{\dagger}$ & $<\mathrm{LOQ}^{\dagger}$
\end{tabular}

* Limit of quantitation in 2002: $60 \mu \mathrm{g} / \mathrm{kg}$; + Limit of quantitation in 2005: $15 \mu \mathrm{g} / \mathrm{kg}$; $¥$ Limit of quantitation in 2002: $30 \mu \mathrm{g} / \mathrm{kg}$

\section{Statistical Analysis}

Acrylamide was included in the statistical models as a continuous variable and as quintiles. Covariables were included in the models if they changed age-adjusted regression coefficients of acrylamide by more than ten percent. A priori, the following variables were considered as potential confounders: age at menarche, duration of oral contraceptive use, 
age at first child birth, parity, age at menopause, duration of postmenopausal hormone use, non-occupational physical activity, body mass index (BMI), height, education level, family history of endometrial cancer (in the endometrial cancer analyses), ovarian or breast cancer (in the ovarian cancer analyses), breast cancer (in the breast cancer analyses), and energy intake (32).

We also checked the following broad categories of foods or nutrients for confounding potential: alcohol consumption, intake of vegetables and fruits, and energy-adjusted intake of saturated fat, trans-unsaturated fatty acids, carbohydrates, and dietary fibre. Smoking status (current vs. not current), the duration of smoking and the number of cigarettes per day were always included in the model, because cigarette smoke is a very important source of acrylamide. Smokers have been shown to have on average four times higher levels of acrylamide-haemoglobin adducts, which is a marker of internal dose of acrylamide, than non-smokers $(33,34)$. Also for this reason, subgroup analyses were done for never-smokers.

The proportional hazards assumption was tested using scaled Schoenfeld residuals. Hazard ratios (HRs) and confidence intervals were obtained through Cox proportional hazards regression with STATA software (package 9.2). Standard errors were estimated using the robust Huber-White sandwich estimator to account for additionalvariance introduced by sampling from the cohort. Tests for dose-response trends were done by fitting ordinal exposure variables as continuous variables.

In order to investigate if the observed associations could be attributed to acrylamide specifically or whether the association was due to other characteristics of acrylamidecontaining foods, models were run with acrylamide and the foods (one at a time) that explained most variance in acrylamide intake.

In order to check for the influence of protopathic bias, the analyses were also done with exclusion of cases occurring in the first two years of follow-up.

Effect modification of the association between acrylamide intake and cancer by other variables was tested using Wald $\chi^{2}$ tests. The variables that were tested for effect modification were selected based on their ability to modify the activity of CYP2E1, the enzyme that converts acrylamide to glycidamide. These variables are diabetes (ever/never diagnosis of diabetes), obesity (BMI $>30 \mathrm{~kg} / \mathrm{m}^{2}$ ), smoking (both duration of smoking in years and number of cigarettes smoked per day), alcohol consumption $(0,>0-5,>5 \mathrm{~g} /$ day $)$, and physical activity $(<30,30-<60,60-<90, \geq 90$ minutes/day) (35-38).

Two-sided $p$ values are reported throughout this paper.

\section{Results}

On average, the subcohort members had a daily acrylamide intake of 21.0 (SD 11.9) $\mu \mathrm{g}$, which corresponds to 0.32 (SD 0.19) $\mu \mathrm{g}$ acrylamide per kg body weight per day. Although coffee was overall the most important contributor to acrylamide intake, Figure 2 shows that not coffee, but Dutch spiced cake was chiefly responsible for the variation in acryl- 
amide intake in this population, followed by coffee, French fries, potato crisps, and cookies.
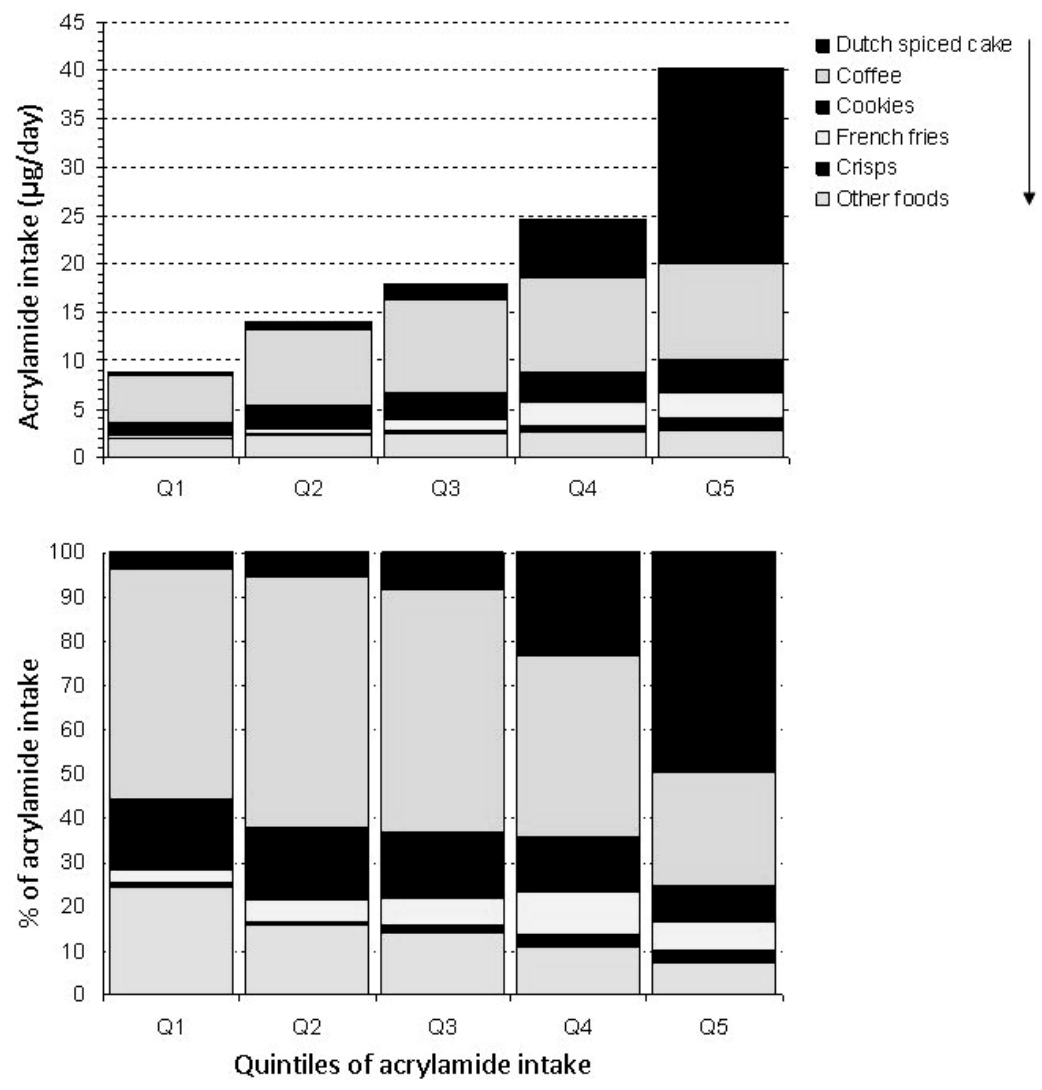

Figure 2: Absolute and relative contribution of foods to the mean daily dietary acrylamide intake of the NLCS subcohort

Table 2 shows the values of the covariables that were assessed for confounding potential across acrylamide intake quintiles. From this Table, it can be concluded that some variables differed considerably between the acrylamide intake categories. There were linear relationships between acrylamide intake and total energy, carbohydrate, saturated fat, trans-unsaturated fatty acids, and fibre intake. There were some significant differences in the non-dietary variables between the quintiles of acrylamide intake, but these variables did not show a linear correlation with acrylamide intake.

In Table 3, the age-adjusted and multivariable-adjusted associations between acrylamide intake and endometrial, ovarian, and breast cancer are shown. There was no statistically significant association between acrylamide as a continuous variable and endometrial cancer, and in the total group of women, there were also no statistically significant associations between quintiles of acrylamide intake and endometrial cancer. However, in the group of never-smoking women, the multivariable-adjusted HR in the fifth 
quintile (HR 1.99; 95\% Cl 1.12-3.52) was statistically significantly higher than that in the lowest quintile of acrylamide intake (P-trend=0.03). In the total group, acrylamide intake as a continuous variable was borderline significantly associated with ovarian cancer (HR 1.11; $95 \% \mathrm{Cl} 0.99-1.25$ for an increment of acrylamide intake with $10 \mu \mathrm{g} \mathrm{day}^{-1}$ ). In the never-smoker subgroup, the association was statistically significant (HR 1.17; $95 \mathrm{Cl} 1.01$ 1.36 for an increment of acrylamide intake with $10 \mu \mathrm{g} \mathrm{day}^{-1}$ ). Also, the HR in the highest quintile of acrylamide intake was statistically significantly higher (total group: HR 1.78 [1.10-2.88]; never-smokers: HR 2.22 [1.20-4.08]) than that of the lowest quintile and the $P$-trend was significant $(P=0.02$ and 0.01 , respectively. There were no indications of an association between acrylamide intake and breast cancer.

Table 4 shows the multivariable-adjusted associations between acrylamide intake and endometrial and ovarian cancer additionally adjusted for coffee, Dutch spiced cake, cookies, French fries and potato crisps consumption, respectively. For both endometrial and ovarian cancer, adjustment for Dutch spiced cake increased the HRs in the highest quintiles and for ovarian cancer it also increased the HR of the continuous acrylamide variable. Adjustment for coffee intake slightly decreased the HRs of the highest quintiles of acrylamide intake and the continuous acrylamide variable for ovarian cancer only. Adjustment for the other foods did not materially change the HRs.

When the cases that occurred during the first two years of follow-up were excluded, the HRs for endometrial and ovarian cancer were slightly higher, whereas the results for breast cancer remained unchanged. None of the interaction terms between acrylamide intake and covariables was statistically significant.

\section{Discussion}

This prospective cohort study provides the first epidemiological indications that dietary acrylamide intake may increase the risk of cancer in humans. For both endometrial and ovarian cancer, a positive association with dietary acrylamide was observed, particularly in the subgroup of never smoking women. Whether the differences between the total group and the never-smokers are due to effect modification by smoking or due to masking of the dietary acrylamide effect by acrylamide or other compounds in tobacco smoke remains to be established. Smoking may induce epoxide hydrolase, which results in enhanced metabolism of glycidamide (33) after which there is less glycidamide left to damage DNA. There was no association between acrylamide intake and postmenopausal breast cancer risk, which is in line with the only other prospective cohort study on this topic (23), although this latter study investigated mainly premenopausal breast cancer. According to the formula of Cai and Zeng for calculating power for case-cohort studies (39), we had a power of $80 \%$ to detect a HR of breast cancer 1.38 or higher for the total group and 1.52 or higher for the never-smokers for comparing the highest quintile of acrylamide intake with the lowest.

The relationship between dietary acrylamide intake and endometrial cancer risk has not been studied before in humans, but the positive association is in line with an increased 


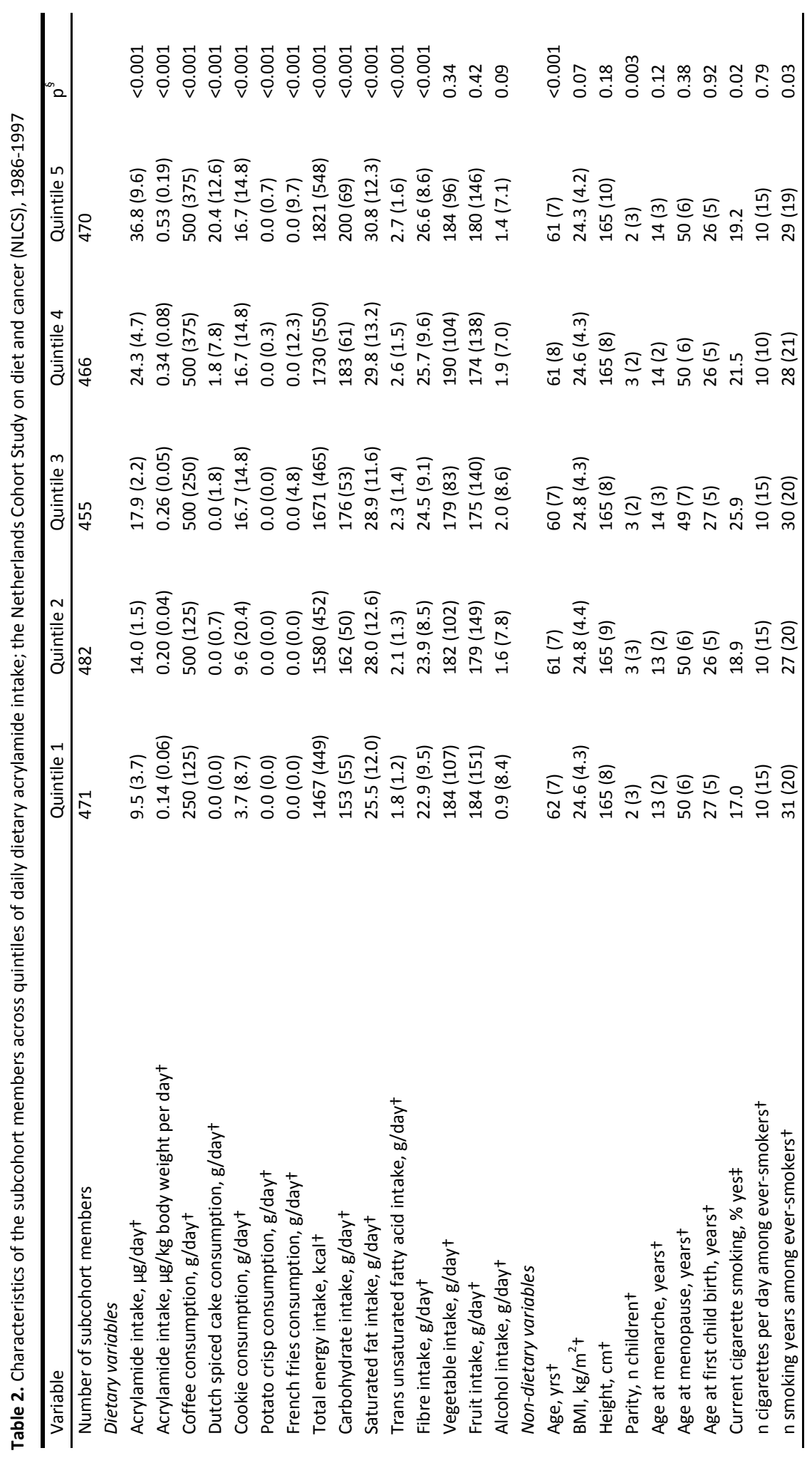




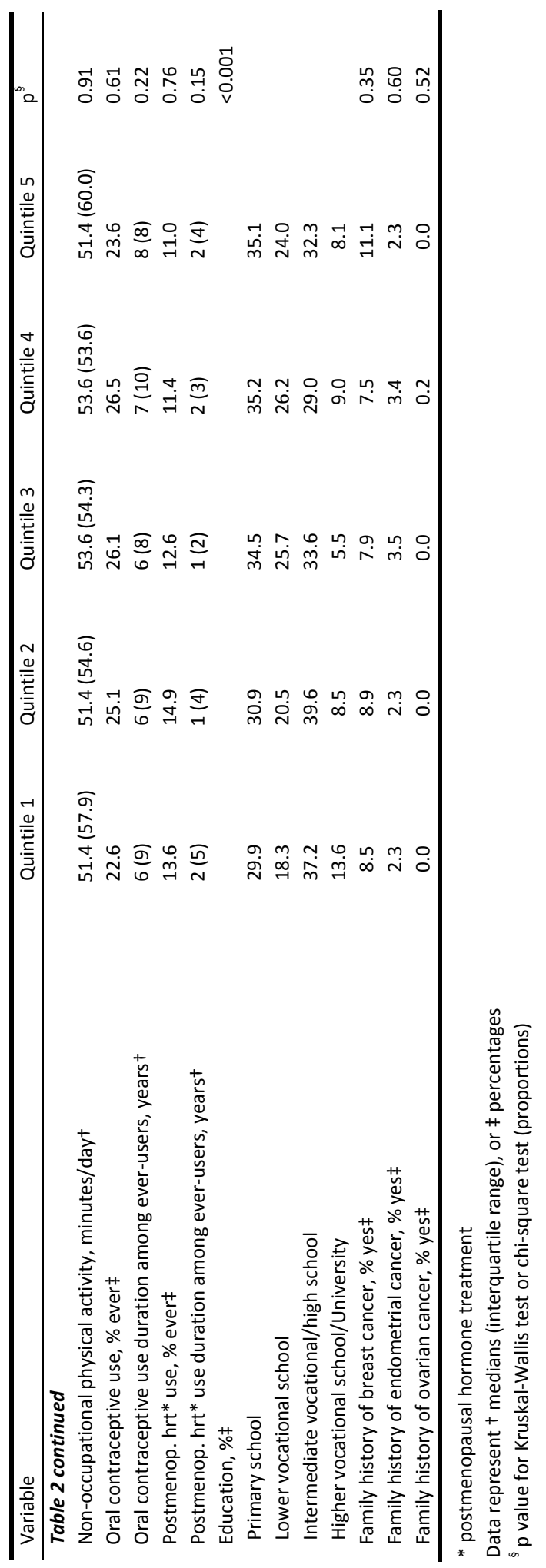




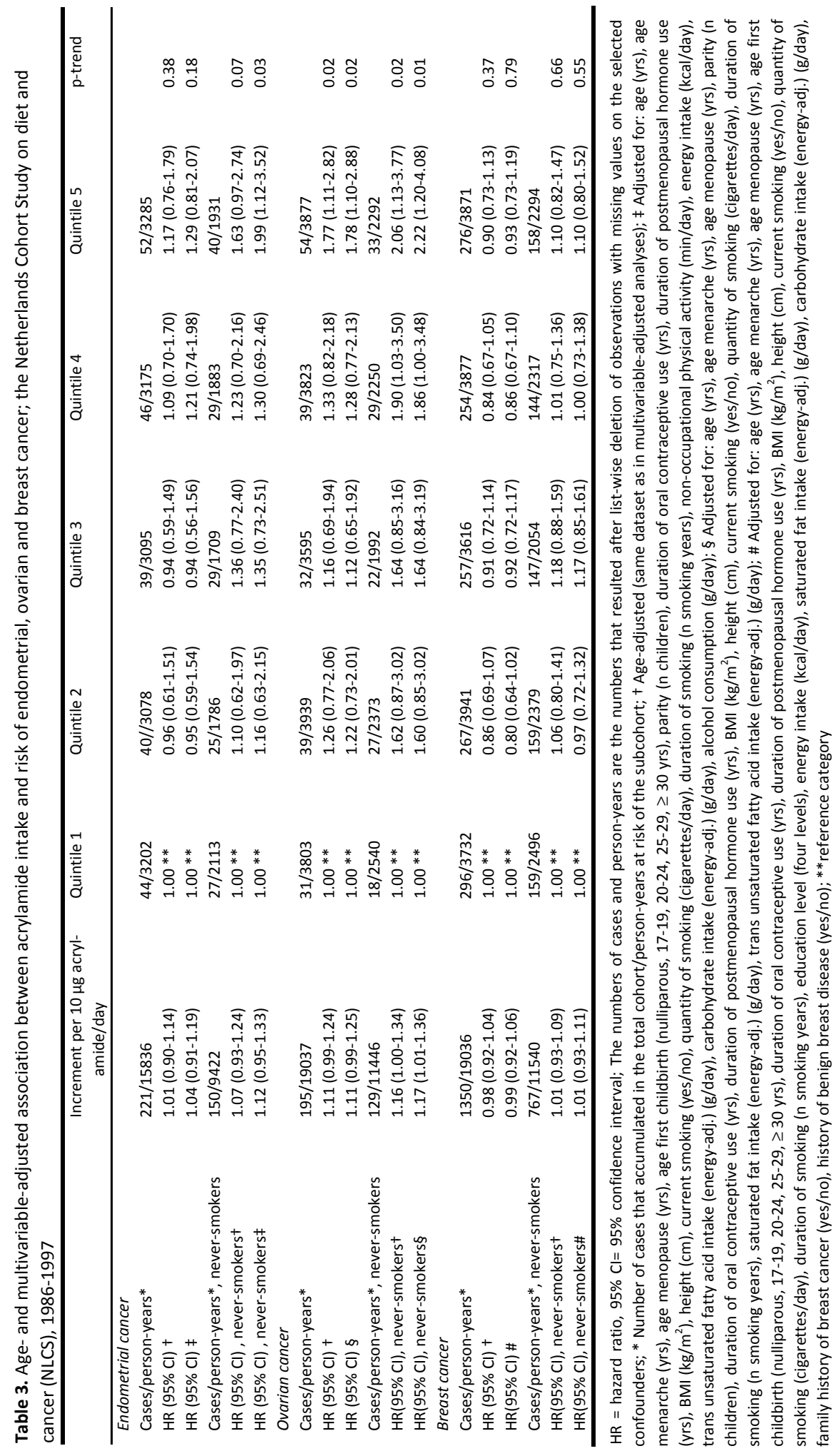




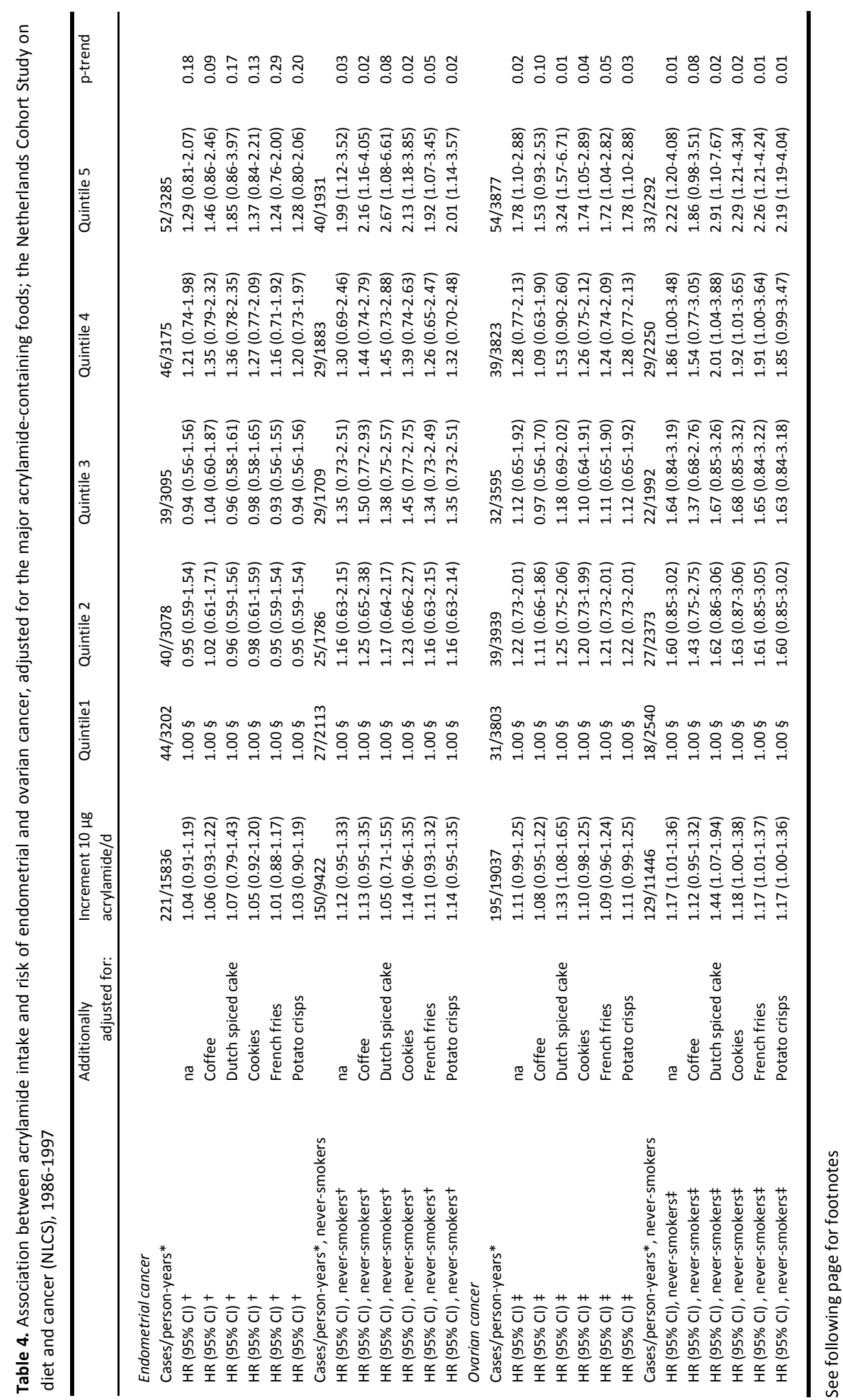




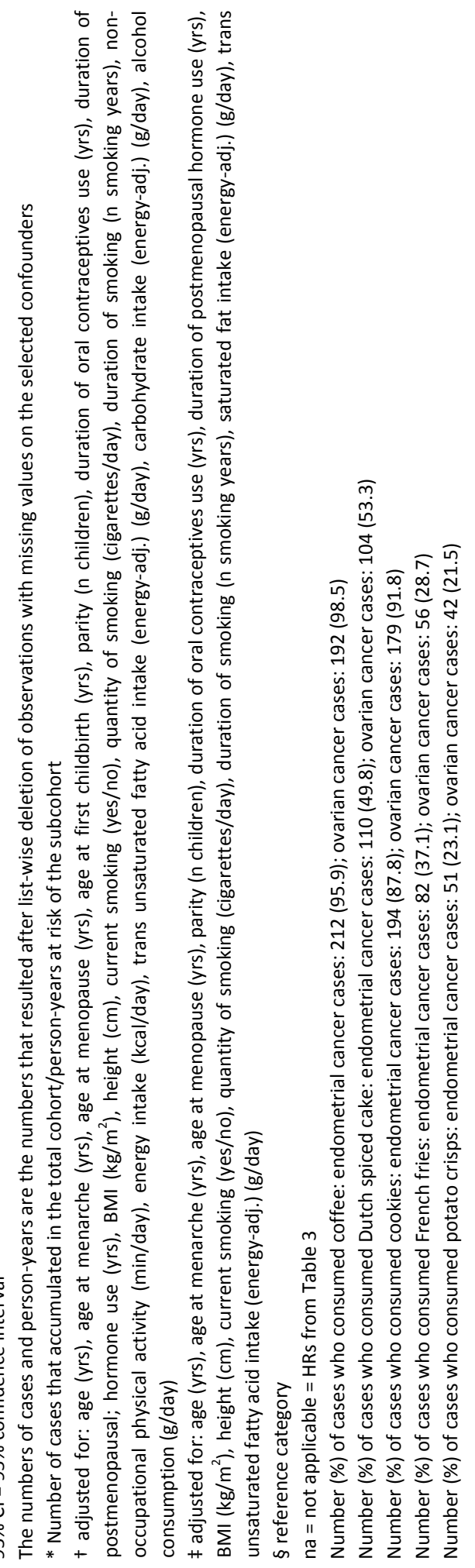


occurrence of uterus tumours in rats after acrylamide exposure (9). The only epidemiological study so far on acrylamide and ovarian cancer risk, a case-control study, showed no association (19). This may be due to preclinical disease affecting food consumption in the case-control study. Generally speaking, case-control studies may suffer from recall bias, but whether this played a role in this particular case-control study is not clear. Recall bias relating to acrylamide itself is unlikely, since the dietary data were gathered before 2002, when acrylamide in food was first reported. However, recall bias may have occurred for

acrylamide-containing foods, such as coffee or French fries. Protopathic bias is not present in our prospective cohort study, in which exclusion of cases in the first two years of follow-up did not alter the conclusions. Another reason for absence of an association in the case-control study might be non-differential misclassification of the acrylamide intake, which biases the effect estimate toward null. Pelucchi et al. based their acrylamide intake assessment on acrylamide concentrations of foods that were not all specifically from the country of the study population and they did not obtain information from the study participants on individual portion sizes, except for fried potatoes.

The acrylamide intake assessment is an important asset of the present study, together with its large study size and prospective nature, which precludes recall bias and makes it less vulnerable to selection bias. We used acrylamide concentrations of foods from the Dutch market only and specifically sampled and analysed foods that were relevant for the NLCS population. Furthermore, the acrylamide measurements were of good quality, which was demonstrated by participation in several international comparison studies of acrylamide analyses $(5,40)$.

Although we have no direct data for acrylamide itself, the reliability and validity of the NLCS food frequency questionnaire (FFQ) for acrylamide can be derived from nutrients that are correlated to acrylamide, namely carbohydrates and dietary fibre. As for validity, the correlation coefficients between the FFQ and a dietary record method were 0.77 for carbohydrates and 0.74 for fibre. For the food groups potatoes, bread, and cakes and cookies, Spearman correlation coefficients were $0.74,0.80$ and 0.65 , respectively (30). Furthermore, the decline of the correlation between the baseline questionnaire and the questionnaire administered after 5 years of follow-up was 0.07 on average among the nutrients that were tested. This indicates that, although the questionnaire was administered only once, it characterizes long-term food intake for over a period of at least five years (29).

Acrylamide intake could be difficult to assess, due to the large range of acrylamide concentrations within foods. Thus, there is potential for non-differential misclassification of acrylamide intake, which would bias risk estimates toward null. We did a validation study to investigate whether using mean acrylamide concentrations in foods can still result in a sound estimate of total acrylamide intake (unpublished results, see footnote ${ }^{1}$ ). We estimated the acrylamide content of 39 Dutch 24-hour duplicate diets that were collected by the Dutch National Institute for Public Health and the Environment in 2004.

1 Unpublished results. Authors: Erik JM Konings, Janneke GF Hogervorst, Linda van Rooij, Leo J Schouten, Eric A Sizoo, Hans P van Egmond, R Alexandra Goldbohm, Piet A van den Brandt, see Chapter 2 of this thesis 
From the diaries of the participants of the duplicate diet study on the amounts of specific foods that were eaten during the 24 hours, the acrylamide content of the 24-hour diets was estimated (= amount of specific food * mean acrylamide level of a specific food), using the mean acrylamide concentrations of the specific foods that were also used for the NLCS acrylamide intake assessment. Furthermore, the acrylamide concentrations of the duplicate diets were chemically analysed and correlated to the estimated acrylamide content, which rendered a Spearman correlation coefficient of 0.82 . This indicates that it is feasible to make a sound rank ordering of the acrylamide intake via a 24-hour diet using these mean acrylamide concentrations for individual foods. For estimating long-term, usual acrylamide intake in the NLCS study, the mean acrylamide concentrations for foods are expected to be even more suitable, as people do not always stick to one particular brand for a certain food, but will switch between brands, at least to some extent. Furthermore, there is also variation in acrylamide concentrations within batches of the same brand. Over time, differences in acrylamide intake of an individual due to different brands and within-brand fluctuations are to some extent averaged out.

This study has a number of limitations. The foods that were sampled and analysed in 2002 and 2005 may not be completely representative of the foods that were on the market in 1986. Since its discovery in food in 2002, industry and science are working together to reduce acrylamide concentrations in foods. Nevertheless, it proves to be quite hard to substantially reduce the concentrations (41). Before 2002, some changes in acrylamide concentrations in foods may have occurred due to changes in production procedures, but never with the intent of lowering acrylamide concentrations. The changes that may have occurred will have resulted in non-differential misclassification of the intake of our cohort, which will then have led to some underestimation of the true associations between acrylamide and the cancer types we studied.

We did not gather information on whether food was bought or prepared at home and have therefore not taken this into account in the analyses. Of the important acrylamidecontaining foods, French fries were most likely to be prepared at home in the NLCS population. However, French fries contributed relatively little to the acrylamide intake and to the variance in acrylamide intake in this cohort. Dutch spiced cake, which is an important acrylamide source in this cohort was not prepared at home. Besides this, the misclassification that comes from the consumption of home-cooked or store-bought foods is probably non-differential and would have biased the risk estimates toward null. Furthermore, it has to be borne in mind that the variation in acrylamide intake in our study was largely due to Dutch spiced cake. Dutch spiced cake resembles gingerbread of the cake-like, non-crusty, type. It does not always contain ginger, but it contains spices and sometimes fruits. Concentrations of acrylamide in Dutch spiced cake (mean: 1018 ppb) are much higher than in plain cake (mean: $<30 \mathrm{ppb}$ ). Due to addition of glucose and fructose containing syrup, it contains a higher amount of reducing sugars than other cakes. In the presence of ammonium hydrogencarbonate, which is often used as a baking agent in spiced cake, the formation of acrylamide is further enhanced (42). Our analyses show that it is not just Dutch spiced cake that is responsible for the observed associations, because the associations between acrylamide intake and endometrial and ovarian cancer were still present after 
adjustment for spiced cake, and also in the subgroup of non-consumers of spiced cake (results for the latter analysis not shown).

The acrylamide-associated hazard ratios that we have observed in this study are considerably higher than the relative risks for dietary acrylamide intake that were calculated based upon linear extrapolation of the cancer incidence observed in animal studies (43) and that led some epidemiologists to conclude that epidemiological studies probably do not have the statistical power to detect an association between dietary acrylamide and cancer. However, the results of extrapolating the high acrylamide dosages that were administered to animals to the low dosages that humans are exposed to through food are uncertain. The activity of enzymes in the metabolism of acrylamide may be either higher or lower at low doses and humans may differ from animals in their ability to handle acrylamide or glycidamide. Furthermore, the genotoxic action of glycidamide $(36,44,45)$ is currently adopted as the mechanism on which acrylamide cancer risk assessments are based. However, the fact that the present study has found associations between acrylamide intake and both endometrial and ovarian cancer might indicate that disturbance of hormonal balances may also be at the basis of acrylamide carcinogenesis (46-48). Acrylamide has been shown to bind to many proteins (49), among which may be proteins related to maintenance of hormonal balances. Park et al. have found a synergistic effect of acrylamide and estrogen on morphological transformation of Syrian Hamster Cells (SHE), an endpoint that is widely used in studies on chemical carcinogenesis (49). This indicates that acrylamide may also be carcinogenic through hormonal pathways, for which animals may not be a good model or for which the linear extrapolation of the animal results to the human situation may not be suitable.

The acrylamide intake of the NLCS population is in the lower part of the WHO estimate range of 0.3-0.8 $\mathrm{\mu g}$ per $\mathrm{kg}$ BW per day for developed countries (50). It corresponds well to the intake level that was obtained from a duplicate diet study in Switzerland, which was $0.28 \mu \mathrm{g}$ per kg BW per day (51), but the authors commented that the participants had consumed far less French fries than the average Swiss population. Current populations, especially young people, consume larger amounts of potato crisps and French fries than the NLCS population did. Because of the high acrylamide concentrations of these foods, current dietary intake of acrylamide is assumed to be considerably higher $(4,5,52)$ than the intake in this study. On the other hand, industry and science are investigating ways to reduce acrylamide concentrations in food and have succeeded to some extent for certain foods (53).

If the associations we have observed in this study were found to be causal, acrylamide in food today could prove to be a relevant health hazard. Therefore, we encourage other researchers to prospectively investigate the association between dietary acrylamide intake and cancer in hormone-sensitive organs. 


\section{References}

1. Tareke E, Rydberg P, Karlsson P, Eriksson S, Tornqvist M. Analysis of acrylamide, a carcinogen formed in heated foodstuffs. J Agric Food Chem 2002;50:4998-5006.

2. Summary and conclusions of the sixty-fourth meeting of the Joint FAO/WHO Expert Committee on Food Additives (JECFA): JECFA, 2005.

3. IARC. Monographs on the evaluation of carcinogen risk to humans: some industrial chemicals. Lyon: International Agency for Research on Cancer, 1994.

4. Dybing $\mathrm{E}$, Farmer PB, Andersen $\mathrm{M}$, et al. Human exposure and internal dose assessments of acrylamide in food. Food Chem Toxicol 2005;43:365-410.

5. Konings EJ, Baars AJ, van Klaveren JD, et al. Acrylamide exposure from foods of the Dutch population and an assessment of the consequent risks. Food Chem Toxicol 2003;41:1569-79.

6. Mottram DS, Wedzicha BL, Dodson AT. Acrylamide is formed in the Maillard reaction. Nature 2002;419:4489.

7. Stadler RH, Blank I, Varga N, et al. Acrylamide from Maillard reaction products. Nature 2002;419:449-50.

8. Taeymans D, Wood J, Ashby P, et al. A review of acrylamide: an industry perspective on research, analysis, formation, and control. Crit Rev Food Sci Nutr 2004;44:323-47.

9. Johnson KA, Gorzinski SJ, Bodner KM, et al. Chronic toxicity and oncogenicity study on acrylamide incorporated in the drinking water of Fischer 344 rats. Toxicol Appl Pharmacol 1986;85:154-68.

10. Bull RJ, Robinson M, Laurie RD, et al. Carcinogenic effects of acrylamide in Sencar and A/J mice. Cancer Res 1984;44:107-11.

11. Bull RJ, Robinson M, Stober JA. Carcinogenic activity of acrylamide in the skin and lung of Swiss-ICR mice. Cancer Lett 1984;24:209-12.

12. Friedman MA, Dulak LH, Stedham MA. A lifetime oncogenicity study in rats with acrylamide. Fundam Appl Toxicol 1995;27:95-105.

13. Marsh GM, Lucas L, Youk AO, Schall LC. Mortality patterns among workers exposed to acrylamide: 1994 follow up. Occup Environ Med 1999;56:181-90.

14. Schulz MR, Hertz-Picciotto I, van Wijngaarden E, Hernandez JC, Ball LM. Dose-response relation between acrylamide and pancreatic cancer. Occup Environ Med 2001;58:609.

15. Sobel W, Bond GG, Parsons TW, Brenner FE. Acrylamide cohort mortality study. Br J Ind Med 1986;43:7858.

16. Collins JJ, Swaen GM, Marsh GM, Utidjian HM, Caporossi JC, Lucas LJ. Mortality patterns among workers exposed to acrylamide. J Occup Med 1989;31:614-7.

17. Swaen GM, Haidar S, Burns CJ, et al. Mortality study update of Acrylamide workers. Occup Environ Med 2007;64:396-401

18. Marsh GM, Youk AO, Buchanich JM, Kant IJ, Swaen G. Mortality patterns among workers exposed to acrylamide: updated follow up. J Occup Environ Med 2007;49:82-95.

19. Pelucchi C, Galeone C, Levi F, et al. Dietary acrylamide and human cancer. Int J Cancer 2006;118:467-71.

20. Mucci LA, Dickman PW, Steineck G, Adami HO, Augustsson K. Dietary acrylamide and cancer of the large bowel, kidney, and bladder: absence of an association in a population-based study in Sweden. Br J Cancer 2003;88:84-9.

21. Mucci LA, Lindblad P, Steineck G, Adami HO. Dietary acrylamide and risk of renal cell cancer. Int J Cancer 2004;109:774-6.

22. Mucci LA, Adami HO, Wolk A. Prospective study of dietary acrylamide and risk of colorectal cancer among women. Int J Cancer 2006;118:169-73.

23. Mucci LA, Sandin S, Balter K, Adami HO, Magnusson C, Weiderpass E. Acrylamide intake and breast cancer risk in Swedish women. Jama 2005;293:1326-7.

24. Zhang M, Yang ZY, Binns CW, Lee AH. Diet and ovarian cancer risk: a case-control study in China. Br J Cancer 2002;86:712-7.

25. Michels KB, Rosner BA, Chumlea WC, Colditz GA, Willett WC. Preschool diet and adult risk of breast cancer. Int J Cancer 2006;118:749-54.

26. van den Brandt PA, Goldbohm RA, van 't Veer P, Volovics A, Hermus RJ, Sturmans F. A large-scale prospective cohort study on diet and cancer in The Netherlands. J Clin Epidemiol 1990;43:285-95.

27. Goldbohm RA, van den Brandt PA, Dorant E. Estimation of the coverage of Dutch municipalities by cancer registries and PALGA based on hospital discharge data. Tijdschr Soc Gezondheidsz 1994;72:80-84.

28. van den Brandt PA, Schouten LJ, Goldbohm RA, Dorant E, Hunen PM. Development of a record linkage protocol for use in the Dutch Cancer Registry for Epidemiological Research. Int J Epidemiol 1990;19:553-8. 
29. Goldbohm RA, van den Brandt PA, Brants HA, et al. Validation of a dietary questionnaire used in a largescale prospective cohort study on diet and cancer. Eur J Clin Nutr 1994;48:253-65.

30. Goldbohm RA, van 't Veer P, van den Brandt PA, et al. Reproducibility of a food frequency questionnaire and stability of dietary habits determined from five annually repeated measurements. Eur J Clin Nutr 1995;49:420-9.

31. Database of the European Commission Institute for Reference Materials and Measurements (IRMM) (accessed August 3, 2006, at http://www.irmm.jrc.be/html/activities/acrylamide/database.htm).

32. Schottenfeld D, Fraumeni JF, Jr. Cancer Epidemiology and Prevention. New York City: Oxford University Press, 2006.

33. Schettgen T, Rossbach B, Kutting B, Letzel S, Drexler H, Angerer J. Determination of haemoglobin adducts of acrylamide and glycidamide in smoking and non-smoking persons of the general population. Int J Hyg Environ Health 2004;207:531-9.

34. Bergmark E. Hemoglobin adducts of acrylamide and acrylonitrile in laboratory workers, smokers and nonsmokers. Chem Res Toxicol 1997;10:78-84.

35. Ardies CM, Smith TJ, Kim S, Yang CS. Induction of 4-(methylnitrosamino)-1-(3-pyridyl)-1-butanone (NNK) activation in rat lung microsomes by chronic ethanol consumption and repeated running exercise. Cancer Lett 1996;103:209-18.

36. Ghanayem BI, Witt KL, Kissling GE, Tice RR, Recio L. Absence of acrylamide-induced genotoxicity in CYP2E1null mice: evidence consistent with a glycidamide-mediated effect. Mutat Res 2005;578:284-97.

37. Howard LA, Micu AL, Sellers EM, Tyndale RF. Low doses of nicotine and ethanol induce CYP2E1 and chlorzoxazone metabolism in rat liver. J Pharmacol Exp Ther 2001;299:542-50.

38. Wang Z, Hall SD, Maya JF, Li L, Asghar A, Gorski JC. Diabetes mellitus increases the in vivo activity of cytochrome P450 2E1 in humans. Br J Clin Pharmacol 2003;55:77-85.

39. Cai J, Zeng D. Sample size/power calculation for case-cohort studies. Biometrics 2004;60:1015-24.

40. Wenzl T, Karasek L, Rosen J, et al. Collaborative trial validation study of two methods, one based on high performance liquid chromatography-tandem mass spectrometry and on gas chromatography-mass spectrometry for the determination of acrylamide in bakery and potato products. J Chromatogr A 2006;1132:211-8.

41. Stadler RH. Acrylamide formation in different foods and potential strategies for reduction. Adv Exp Med Biol 2005;561:157-69.

42. Amrein TM, Andres L, Manzardo GG, Amado R. Investigations on the promoting effect of ammonium hydrogencarbonate on the formation of acrylamide in model systems. J Agric Food Chem 2006;54:1025361.

43. Dybing E, Sanner T. Risk assessment of acrylamide in foods. Toxicol Sci 2003;75:7-15.

44. Gamboa da Costa G, Churchwell MI, Hamilton LP, et al. DNA adduct formation from acrylamide via conversion to glycidamide in adult and neonatal mice. Chem Res Toxicol 2003;16:1328-37.

45. Doerge DR, da Costa GG, McDaniel LP, Churchwell MI, Twaddle NC, Beland FA. DNA adducts derived from administration of acrylamide and glycidamide to mice and rats. Mutat Res 2005;580:131-41.

46. Akhmedkhanov A, Zeleniuch-Jacquotte A, Toniolo P. Role of exogenous and endogenous hormones in endometrial cancer: review of the evidence and research perspectives. Ann N Y Acad Sci 2001;943:296-315.

47. Cramer DW, Welch WR. Determinants of ovarian cancer risk. II. Inferences regarding pathogenesis. J Natl Cancer Inst 1983;71:717-21.

48. Exon JH. A review of the toxicology of acrylamide. J Toxicol Environ Health B Crit Rev 2006;9:397-412.

49. Park J, Kamendulis LM, Friedman MA, Klaunig JE. Acrylamide-induced cellular transformation. Toxicol Sci 2002;65:177-83.

50. Health implications of acrylamide in food. Report of a Joint FAO/WHO Consultation.: FAO/WHO, 2002.

51. Swiss Federal Office of Public Health. Assessment of acrylamide intake by duplicate diet study. 2002. (Accessed August 3, 2006, at http://www.bag.admin.ch).

52. Matthys C, Bilau M, Govaert Y, Moons E, De Henauw S, Willems JL. Risk assessment of dietary acrylamide intake in Flemish adolescents. Food Chem Toxicol 2005;43:271-8.

53. Acrylamide Status Report December 2004: a summary of the efforts and progress achieved to date by the European food and drink industry (CIAA) in lowering levels of acrylamide in food. Bruxelles: CIAA, 2004. 



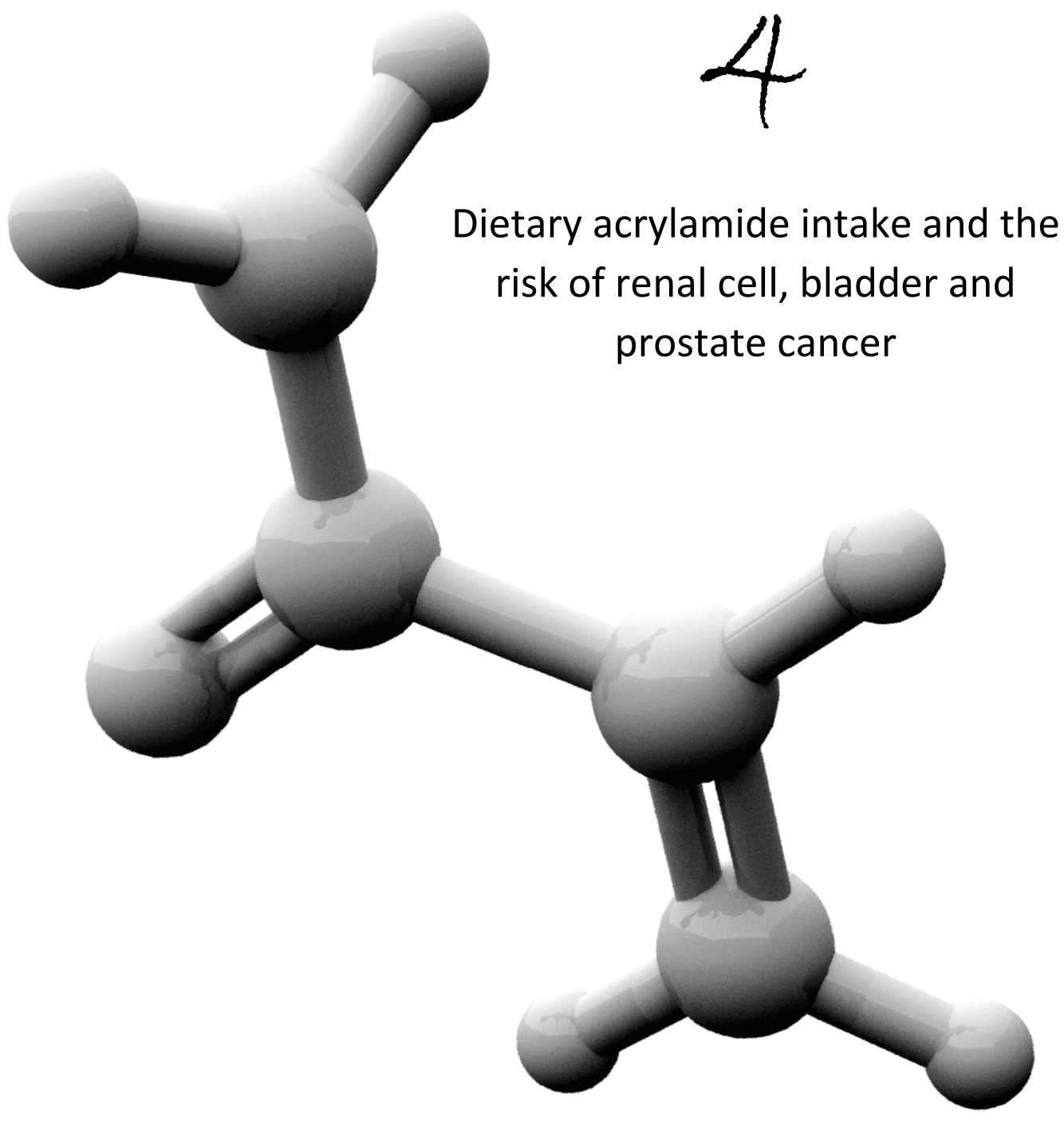

Janneke G.F. Hogervorst

Leo J. Schouten

Erik J.M. Konings

R. Alexandra Goldbohm

Piet A. van den Brandt 


\section{Abstract}

Background Acrylamide, a probable human carcinogen, was recently detected in various heat-treated carbohydrate-rich foods. Epidemiological studies on the relationship with cancer risk have been few and largely negative.

Objective To prospectively examine the association between dietary acrylamide intake and renal cell, bladder, and prostate cancers.

Design The Netherlands Cohort Study on diet and cancer includes 120852 men and women aged 55-69 years. At baseline (1986), a random subcohort of 5000 participants was selected, using a case-cohort analysis approach using Cox proportional hazards analysis. Acrylamide intake was assessed with a food-frequency questionnaire at baseline and was based on chemical analysis of all relevant Dutch foods.

Results After 13.3 y of follow-up, 339, 1210, and 2246 cases of renal cell, bladder, and prostate cancer, respectively, were available for analysis. Compared with the lowest quintile of acrylamide intake (mean intake $=9.5 \mu \mathrm{g} /$ day), multivariable-adjusted hazard ratios (HRs) for renal cell, bladder, and prostate cancer in the highest quintile (mean intake $=40.8 \mu \mathrm{g} /$ day) were $1.59(95 \% \mathrm{Cl} 1.09,2.30 ; \mathrm{p}$-trend 0.04), 0.91 (95\% Cl 0.73, 1.15; p-trend 0.60$)$, and $1.06(95 \% \mathrm{Cl} 0.87,1.30 ; \mathrm{p}$-trend 0.69$)$, respectively. There was an inverse non-significant trend for advanced prostate cancer risk in never-smokers.

Conclusions We found some indications for a positive association between dietary acrylamide and renal cell cancer risk. There were no positive associations with bladder and prostate cancer risk. 


\section{Introduction}

Acrylamide $\left(\mathrm{C}_{3} \mathrm{H}_{5} \mathrm{NO}\right)$ is a small hydrophilic molecule that polymerises readily in the presence of an initiator because of the double bond between the first and second C-atoms, which makes it a versatile industrial chemical. In 1994, the International Agency for Research on Cancer classified acrylamide as a probable human carcinogen, on the basis of its carcinogenicity in rodents (1). Before 2002, acrylamide exposure was thought to occur mainly through occupational exposure, also through cigarette smoke and to a minor extent through the consumption of drinking water and the use of cosmetics. However, in 2002 Swedish scientists reported its presence in carbohydrate-rich foods that were produced at high temperatures, such as French fries and potato crisps (2). This finding alarmed the scientific community, particularly because acrylamide is present in foods at considerably higher concentrations than other well-known food carcinogens, such as polycyclic aromatic hydrocarbons and ethyl carbamate (3).

Shortly after its discovery in food, it was shown that acrylamide is formed in Maillard browning reactions in which amino acids, particularly asparagine, react with reducing sugars at high temperatures $\left(>120^{\circ} \mathrm{C}\right)(4,5)$.

The mechanism by which acrylamide causes cancer in laboratory animals and by which it may cause cancer in humans is yet unclear (6); both genotoxic and non-genotoxic pathways have been suggested. Acrylamide itself and its epoxide metabolite glycidamide, which is generated by cytochrome P4502E1 (CYP2E1), are clastogenic and glycidamide forms DNA adducts. As for possible non-genotoxic pathways, acrylamide reacts with glutathione and may thus influence the redox status of cells and gene transcription, or it may interfere with DNA repair or hormonal balances (6).

Animal studies have shown positive dose-response relationships between acrylamide exposure and cancer in multiple organs in both mice and rats (7-10); included among those organs were several hormone-sensitive organs, such as the mammary glands and the uterus. Studies on occupational acrylamide exposure have been negative so far, apart from a finding of a greater risk of pancreatic cancer, but that finding was based on a small number of cases (11-16). Dietary acrylamide intake and its relation with various types of cancer has been studied in few case-control studies (17-19) and only two prospective cohort studies $(20,21)$. One prospective cohort study by our group showed a positive association between dietary acrylamide intake and endometrial and ovarian cancers, but not postmenopausal breast cancer (22).

Because the acrylamide molecule is small and hydrophilic, it passively diffuses throughout the whole body (23). For this reason, all tissues are theoretically targets for acrylamide carcinogenesis. In the present prospective cohort study, we investigated the relation between dietary acrylamide and risk of cancer of the kidney, bladder and prostate. Prostate cancer was of particular interest because of its hormonal etiology. 


\section{Subjects and methods}

\section{Study cohort, cases, and follow-up}

The Netherlands Cohort Study on diet and cancer (NLCS) began in September 1986 with the enrolment of 58279 men and 62573 women aged 55-69. At baseline, the participants completed a self-administered questionnaire on 150 food items and on possible risk factors for cancer, such as smoking, occupation, and physical activity. Participants were informed that, by returning a completed questionnaire, they gave their consent to participate in a study of the aetiology of cancer in relation to diet. The study protocol was approved by the Medical Ethics Committees of the University Hospital Maastricht and TNO Nutrition.

Data processing and analysis were based on the case-cohort approach, in which the cases were enumerated for the entire cohort (providing the numerator information for estimating incidence rates), whereas the accumulated person-years for the entire cohort were estimated from a subcohort of 5000 men and women randomly sampled from the entire cohort at baseline (providing the denominator information for estimating incidence rates). Thus, all of the incident cases in the total cohort were used in the analyses. The size of the subcohort was based on calculations that were described previously (24). Since the start of the study, vital status information was obtained from the subcohort at regular intervals. Incident cases in the total cohort have been detected by annual computerised record linkages to the regional cancer registries and the Netherlands Pathology Registry. The completeness of cancer follow-up through linkage with the cancer registries was estimated to be at least $96 \%$ (25). The follow-up of the subcohort was nearly $100 \%$ complete (only two male subcohort members were lost to follow-up) at the end of the followup period. Further details on the design of the study and methods of follow-up were presented elsewhere $(24,26-28)$.

The analyses are based on 13.3 years of follow-up, from September 1986 through December 1999. After 13.3 years of follow-up, there were 402, 1381, and 2599 incident, epithelial, microscopically confirmed, primary cases of renal cell ([ICD-O]-3:C64), bladder ([ICD-O]-3:C67), and prostate ([ICD-O]-3:C61) carcinoma, respectively. There were 759 invasive bladder cancer cases and 734 papillary, non-invasive bladder cancer cases. Some bladder cancer cases had both an invasive and a papillary, non-invasive tumour. As for prostate cancer, 947 cases, defined by tumour $(T)$ and metastasis $(M)$ status, were of the advanced prostate cancer type [which entails stage III prostate cancer (T3-4, MO) and stage IV prostate cancer (T0-4, M1); American Joint Committee on Cancer Classification], and 1546 were of the localised type (TO-2, MO), whereas 106 cases had tumours of unknown stage.

Cases and subcohort members were excluded from analysis if they had been diagnosed with cancer (other than skin cancer) at baseline and if their dietary data were incomplete or inconsistent. The selection and exclusion steps that resulted in the numbers of cases and subcohort members that were available for analysis are shown in Figure 1. 
Netherlands Cohort Study on diet and cancer

$102852 \partial+$

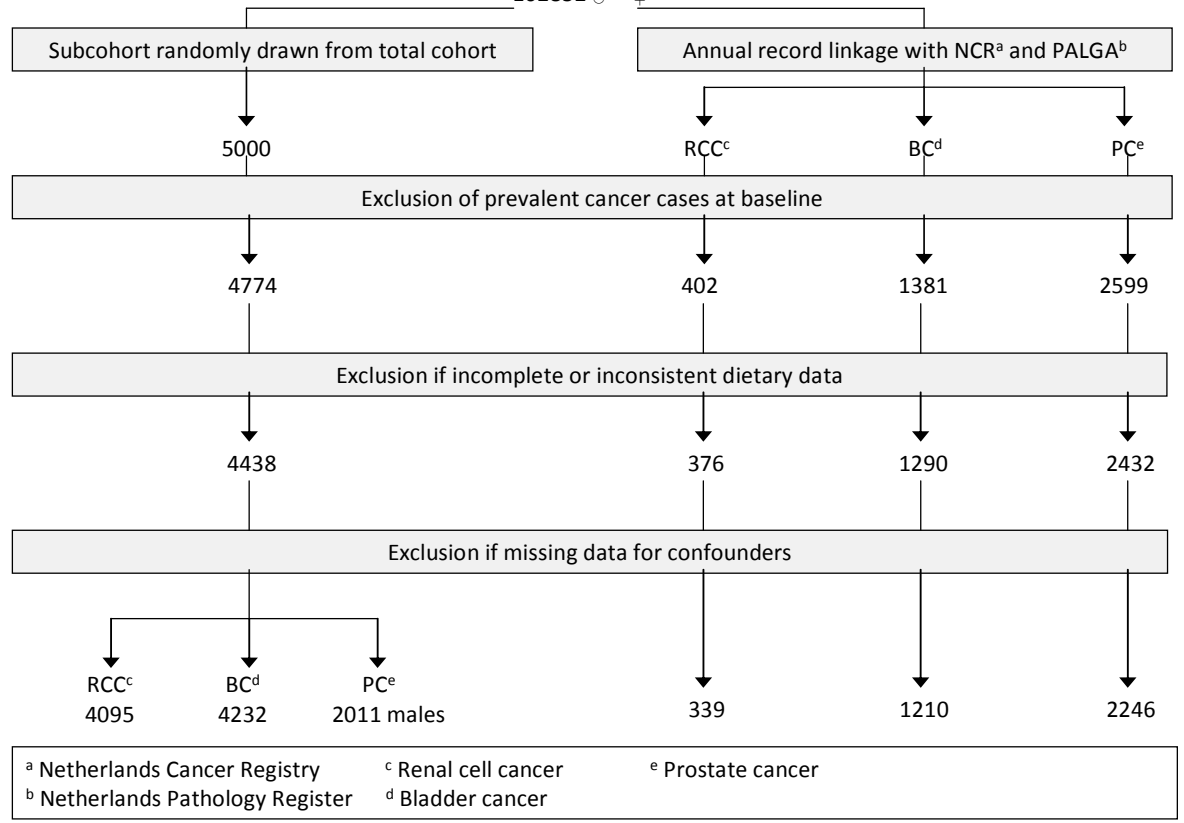

Figure 1: Flow diagram of subcohort members and cases on whom the analyses were based

\section{Acrylamide intake assessment}

The NLCS food-frequency questionnaire (FFQ) contained questions on 150 food items (27). The acrylamide intake was estimated from the mean acrylamide concentration of food items, and the frequency of consumption and portion size of the food items.

To obtain an intake estimate representative of the Dutch diet, we used data on acrylamide concentrations in food products on the Dutch market. In 2002, the Dutch Food and Consumer Product Safety Authority used an elaborate sampling scheme to analyse acrylamide concentrations in various foods, such as bread, French fries, pastry and cake, Dutch spiced cake, crisps, cornflakes, meat, fish, and several beverages (29). In 2005, more foods were analysed to specifically accommodate the estimation of the acrylamide intake of the NLCS cohort; this series consisted of bread, rusk, specific types of cookies, rye bread, crisp bread, pastry and cake, chocolate and chocolate milk, nuts and salty snacks, peanut butter, and coffee (30). Bread was sampled and analysed again in 2005, because the quantitation limit of the analytical method had decreased from $30 \mathrm{ppb}$ in 2002 to $15 \mathrm{ppb}$ in 2005 , and that change offered the opportunity to more accurately estimate the acrylamide content of bread.

The foods that were used in the acrylamide intake assessment were assigned the mean value of the acrylamide values per food or a value one-half the quantitation limit when concentrations were lower than the quantitation limit (because even those foods probably do contain some acrylamide). Other foods (ie, meats, cooked and raw vegetables, and dairy products) were assigned the value zero. Because acrylamide concentra- 
tions in individual foods vary considerably, our group has done a validation study to investigate whether using mean acrylamide concentrations in foods results in a sound estimate of total acrylamide intake (see Chapter 2 of this thesis). The acrylamide content of 39 Dutch 24-hour duplicate diets from 2004 was estimated by using the menu list (on which amounts of individual foods in the 24-hour diets were listed) that the participants of the duplicate diet study filled in for their duplicate diets, and the same mean acrylamide concentrations for foods that were used in the NLCS study. Next, the acrylamide concentrations of the duplicate diets were chemically analysed and correlated to the estimated acrylamide content, which rendered a Spearman correlation coefficient of 0.82 . This indicates that it is feasible to make a sound rank ordering of the acrylamide intake via a 24-hour diet by using these mean acrylamide concentrations for individual foods. The acrylamide concentrations of foods that were used in the acrylamide intake assessment are shown in Table 1.

Table 1: Acrylamide concentrations in foods, used for the NLCS acrylamide intake assessment

\begin{tabular}{lcccc}
\hline & n samples & $\begin{array}{l}\text { mean } \\
(\mu \mathrm{g} / \mathrm{kg})\end{array}$ & $\begin{array}{l}\text { minimum } \\
(\mu \mathrm{g} / \mathrm{kg})\end{array}$ & $\begin{array}{c}\text { maximum } \\
(\mu \mathrm{g} / \mathrm{kg})\end{array}$ \\
\hline Potato crisps & 40 & 1249 & 310 & 2800 \\
Dutch spiced cake & 13 & 1018 & 260 & 1410 \\
French fries & 33 & 351 & $<\mathrm{LOQ}^{*}$ & 1220 \\
Salty snacks & 12 & 277 & 45 & 867 \\
Crisp bread & 12 & 229 & 15 & 914 \\
Cookies & 20 & 204 & 10 & 829 \\
Cornflakes & 12 & 121 & $<\mathrm{LOQ}^{*}$ & 300 \\
Peanut butter & 2 & 113 & 107 & 118 \\
Chocolate & 6 & 60 & 22 & 116 \\
Nuts & 8 & 33 & $<\mathrm{LOQ}^{\dagger}$ & 83 \\
Rusk & 3 & 25 & 16 & 33 \\
Rye bread & 13 & 24 & $<\mathrm{LOQ}^{\dagger}$ & 60 \\
Sweet pastry & 19 & 18 & $<\mathrm{LOQ}^{\dagger}$ & 111 \\
Coffee & 9 & 17 & $<\mathrm{LOQ}^{\dagger}$ & 28 \\
Chocolate milk & 4 & $<\mathrm{LOQ}^{\dagger}$ & $<\mathrm{LOQ}^{\dagger}$ & $<\mathrm{LOQ}^{\dagger}$ \\
Bread & 22 & $<\mathrm{LOQ}^{\dagger}$ & $<\mathrm{LOQ}^{\dagger}$
\end{tabular}

Acrylamide in coffee was analysed in brewed filtered coffee that was prepared according to general Dutch preparation instructions ( $7 \mathrm{~g}$ of coffee powder/125 ml of water). Acrylamide measurements in cookies were done in several types of cookies known to be eaten most frequently by the population comparable to the NLCS, according to information from the development phase of the questionnaire. Thus, the acrylamide concentration for cookies was based on the acrylamide level of the specific types of cookies weighted by the frequency of consumption by a NLCS-comparable population. The same was done for other composite food items, such as bread; * Limit of quantitation in 2002: $60 \mu \mathrm{g} / \mathrm{kg}$; † Limit of quantitation in 2005: $15 \mu \mathrm{g} / \mathrm{kg}$; $¥$ Limit of quantitation in 2002: $30 \mu \mathrm{g} / \mathrm{kg}$

\section{Statistical analysis}

Acrylamide was included in the statistical models as a continuous variable and as a quintile distribution. Furthermore, the fifth quintile was split into two deciles to investigate the highest $10 \%$ of the intake, which is more in line with the intake of current populations in developed countries (31), such as the Netherlands. 
A priori, on the basis of the literature, variables, besides age and sex, were selected for inclusion in the multivariable-adjusted models. For renal cell cancer, those variables were hypertension, body mass index, energy intake, fruit consumption, and vegetable consumption; for bladder cancer, they were vegetable consumption, fruit consumption, tea consumption, and family history of bladder cancer; and for prostate cancer, they were education level, alcohol intake, and family history of prostate cancer (32). Other variables were included in the models if they changed the age- and sex-adjusted hazard ratios (HRs) of acrylamide (expressed as the interval between the $10^{\text {th }}$ and $90^{\text {th }}$ percentile of intake: 27 $\mu \mathrm{g}$ acrylamide/day) by more than ten percent. Different groups of variables were checked for confounding potential on the basis of this change of estimate rule. For renal cell cancer analysis, those variables were education level, physical activity, tea consumption, and family history of renal cell cancer; for bladder cancer, they were body mass index, socioeconomic status, total fluid intake, energy intake, and meat, fish and cheese intakes; and for prostate cancer analysis, they were body mass index, height, physical activity, energy intake, and meat, fish, vegetable, fruit, and dairy consumption. We also checked the following broad categories of foods or nutrients for confounding potential for the three cancer types: alcohol consumption, energy-adjusted intake of saturated fat, transunsaturated fatty acids, carbohydrates, and dietary fibre. Because cigarette smoke is a very important source of acrylamide, smoking status (current or not current), the duration of smoking, and the number of cigarettes per day were included in the model for the total group (but not in the analyses for never-smokers). Smokers have been shown to have, on average, four times the concentrations of acrylamide-haemoglobin adducts -a marker of internal dose of acrylamide- as do non-smokers $(33,34)$. For the same reason, subgroup analyses were done for never-smokers.

The proportional hazards assumption was tested by using scaled Schoenfeld residuals. HRs (and 95\% Cls) were obtained by using Cox proportional hazards regression and STATA software (version 9.2; Stata Corp, College Station, TX). SEs were estimated using the robust Huber-White sandwich estimator to account for additional variance introduced by sampling from the cohort. Tests for dose-response trends were done by fitting the median acrylamide intake for the quintiles of acrylamide intake.

To investigate whether the observed associations could be attributed to acrylamide itself or whether the association was due to other characteristics of acrylamide-containing foods than the acrylamide content, models were run with acrylamide and the foods (one at a time) that explained most variance in acrylamide intake.

Effect modification of the association between acrylamide intake and cancer by other variables was tested by using Wald $\chi^{2}$ tests. The variables that were tested for effect modification were selected on the basis of their ability to modify the activity of CYP2E1, the enzyme that converts acrylamide to glycidamide. Other acrylamide researchers have recommended studying the interaction between dietary acrylamide intake and factors that influence the expression of this enzyme in epidemiological studies $(35,36)$. These variables are diabetes, obesity, smoking, alcohol consumption, and physical activity (35, 37-39). The categories of these variables were a priori based either on existing categories in the NLCS database (physical activity) or on the number of subcohort members within 
the categories, to strive for sufficient participants in each category (number of cigarettes smoked per day, number of smoking years, and alcohol intake).

Throughout, two-sided $\mathrm{p}$ values are reported.

\section{Results}

On average, the subcohort members had a mean (SD) acrylamide intake of 21.8 (12.0) $\mu \mathrm{g} /$ day, which corresponds to $0.30(0.18) \mu \mathrm{g}$ acrylamide per kg body weight per day. Men had a slightly higher absolute intake (absolute: 22.5 (12.1) $\mu \mathrm{g} /$ day) but a slightly lower intake per kg body weight per day $(0.29(0.16) \mu \mathrm{g} / \mathrm{kg}$ body weight/day), than did women (absolute 21.0 (11.9) $\mu \mathrm{g} /$ day); per kg bodyweight: $0.32(0.19) \mu \mathrm{g} / \mathrm{kg}$ body weight/day). Although coffee was overall the most important contributor to acrylamide intake, Figure 2 shows that it was not coffee, but, rather, Dutch spiced cake that was chiefly responsible for the variations in acrylamide intake in the population of the present study; next most responsible were coffee, French fries, potato crisps, and cookies. These findings applied to both men and women.
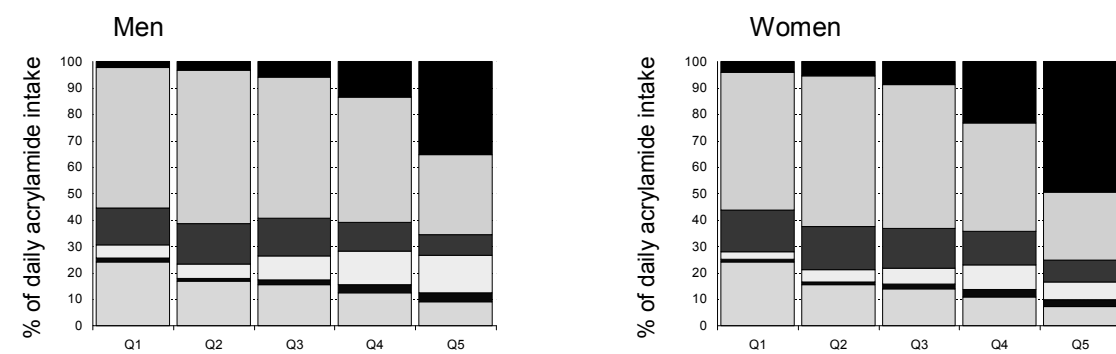

Quintiles of daily acrylamide intake

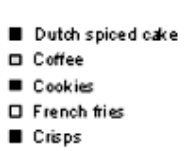

Quintiles of daily acrylamide intake

Crench tri

Men

口 Other foods

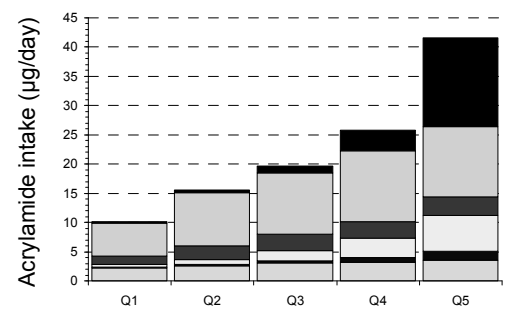

Quintiles of daily acrylamide intake

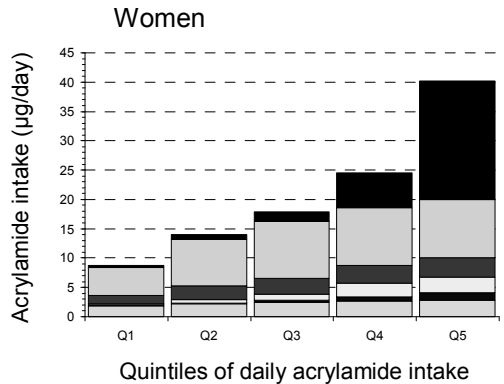

Figure 2. Relative and absolute contribution of foods to the mean daily dietary acrylamide intake of the NLCS subcohort

The values of the covariables that were assessed for confounding potential for the subcohort and for the cases, separately for men and women are shown in Table 2. There were no striking differences between cases and subcohort members for most of the 
variables. Only smoking (current smoking, number of cigarettes per day, and number of smoking years) was more prevalent among bladder cancer cases and among male renal cell cancer cases than among subcohort members.

The age- and multivariable-adjusted associations between acrylamide intake and renal cell cancer risk for men and women combined are shown in Table 3. There was a statistically significant positive association between acrylamide as a continuous variable and renal cell cancer risk in the group of men and women combined (HR 1.10 (95\% Cl: 1.01, 1.21) per each 10 increment in acrylamide intake per day. For men and women separately, the HRs were similar, $1.10(0.98,1.23)$ and $1.11(0.94,1.31)$, respectively. In the group of men and women combined, there was a significant $(p=0.04)$ positive trend from the lowest to the highest quintile of acrylamide intake, with an HR of $1.59(1.09,2.30)$ in the highest quintile compared with the lowest. This pattern was observed in both men and women. There was no association in the subgroup of never-smokers.

The associations between acrylamide intake and bladder cancer risk for men and women combined are presented in Table 4. Acrylamide intake was not associated with bladder cancer risk when male and female never, former and current smokers were combined. Moreover, in the subgroup of participants within the highest decile of acrylamide intake, no greater risk was observed either. However, in the never-smoking subgroup, acrylamide intake tended to be inversely associated with bladder cancer risk ( $p$ trend 0.07), but there was no linear dose-response relation. Stratification of the analyses by sex showed that an inverse association was found in women (although it was not statistically significant and did not have a linear dose-response relation), whereas, in men, the HRs were around 1. The same pattern was found for never-smoking women. Similar results were observed for invasive bladder cancer.

From the data given in Table 5, it can be concluded that there were no indications of a positive association between acrylamide intake and total prostate cancer risk. There was also no greater HR in the highest decile of acrylamide intake. This finding was similar for advanced prostate cancer. However, there was an inverse statistically non-significant ( $p$ for trend $=0.10$ ) dose-response relation between acrylamide intake and advanced prostate cancer risk in never-smokers.

Adjustment for coffee intake slightly decreased the HRs of the highest quintiles of acrylamide intake and the continuous acrylamide variable for renal cell cancer. Adjustment for the other foods did not change the HRs (data not shown).

The results of the analyses (men and women combined) of interactions between acrylamide and possible CYP2E1-influencing variables are shown in Table 6, Table 7, and Table 8. For renal cell cancer (Table 6), there were no significant $p$ values for interaction for any of the possible CYP2E1-influencing variables. In the bladder cancer analyses (Table 7), cigarette smoking (ie, the number of cigarettes smoked per day) significantly modified the association between acrylamide and bladder cancer risk ( $p$ interaction $=0.02$ ), and there was a borderline statistically significant HR of $1.09(1.00,1.19)$ for persons smoking 15 or more cigarettes a day. Obese women had a lower (HR 0.47; 95\% Cl 0.21, 1.05; p interaction 0.03) acrylamide-associated risk of bladder cancer than did non-obese women, although this finding was based on only 16 obese cases (data not shown). The $p$ values for 


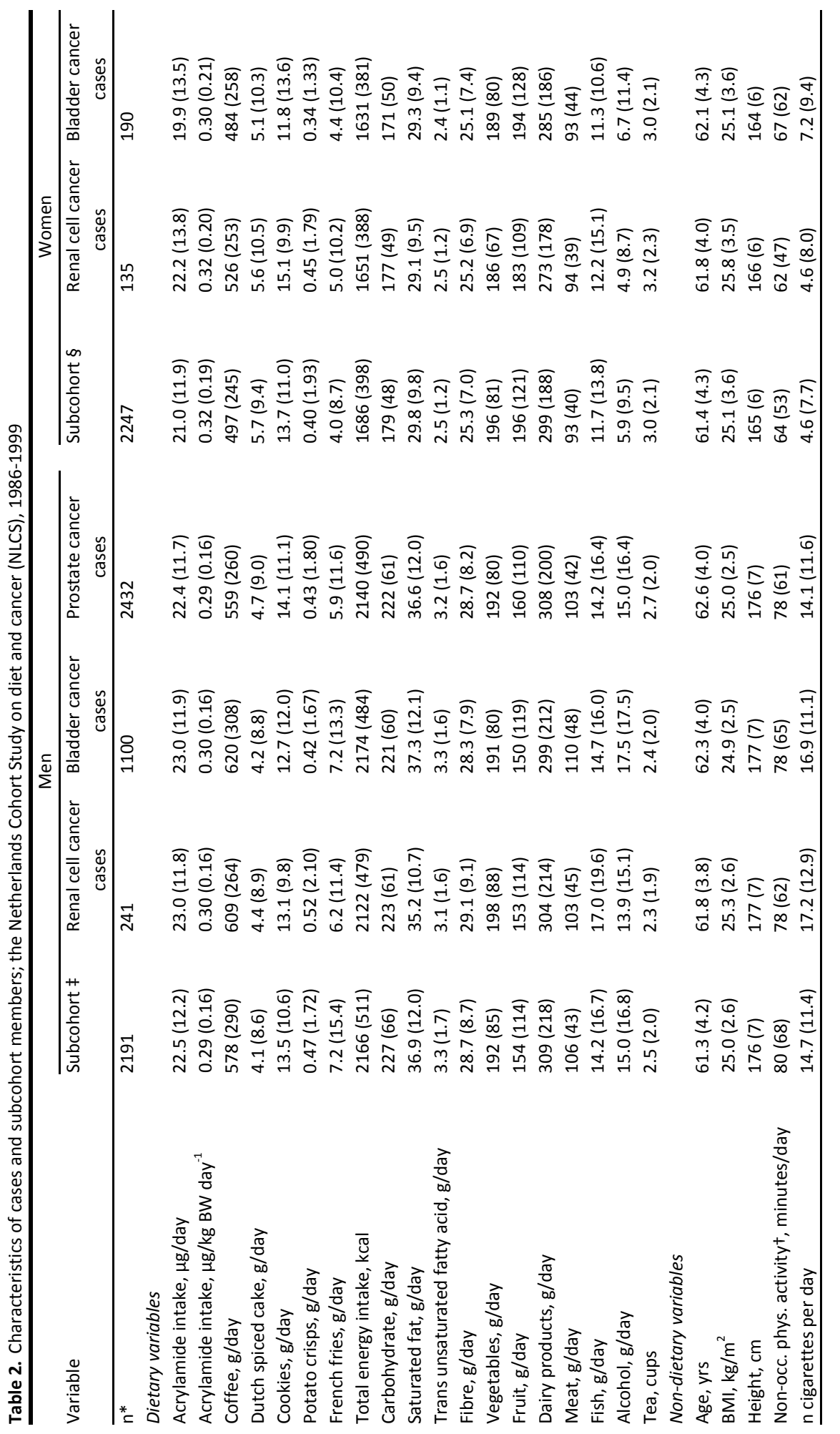




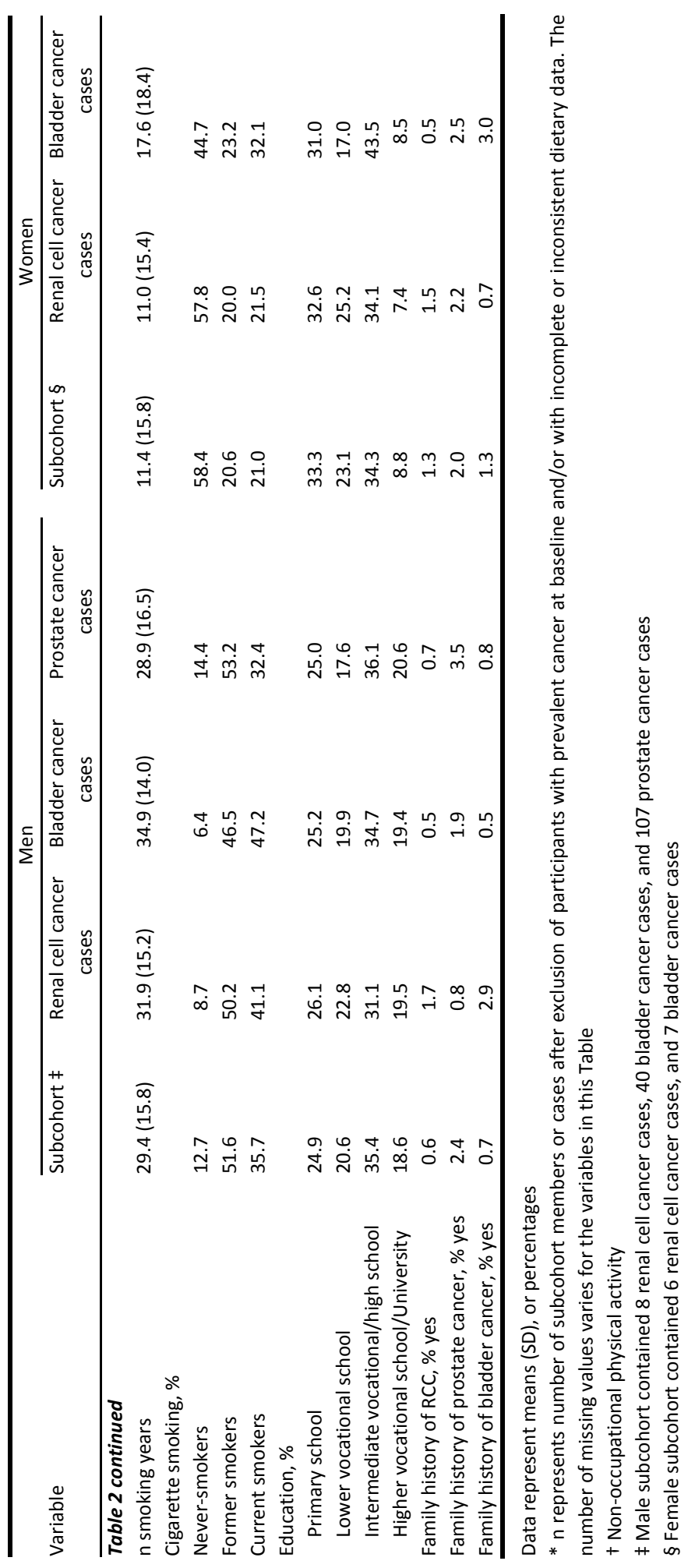




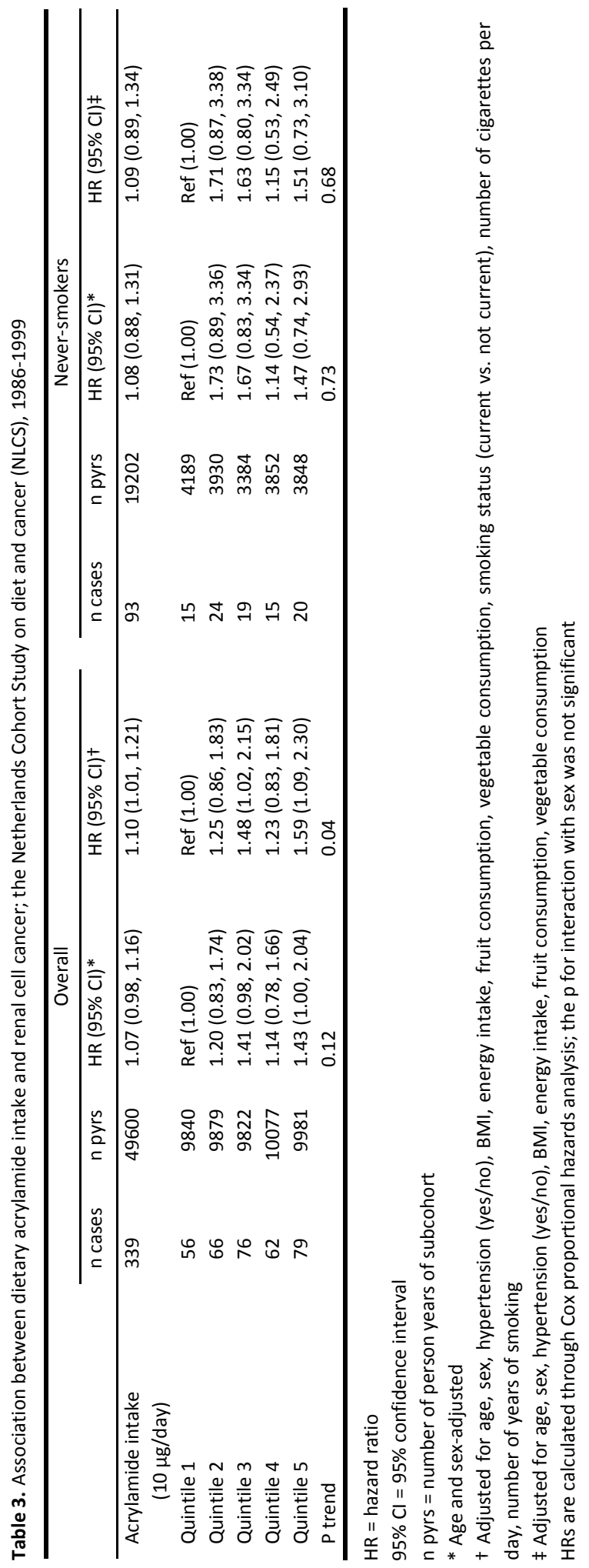




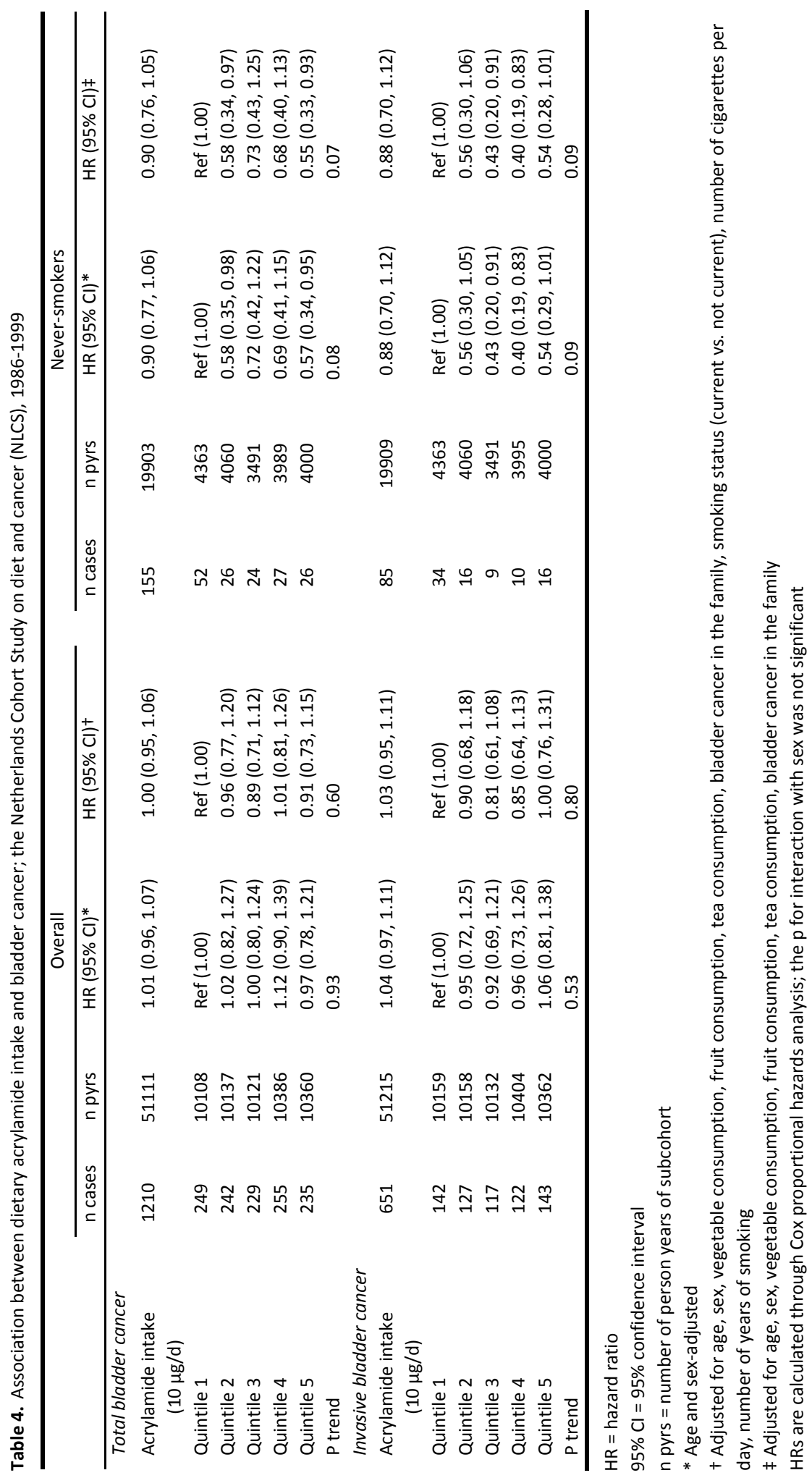




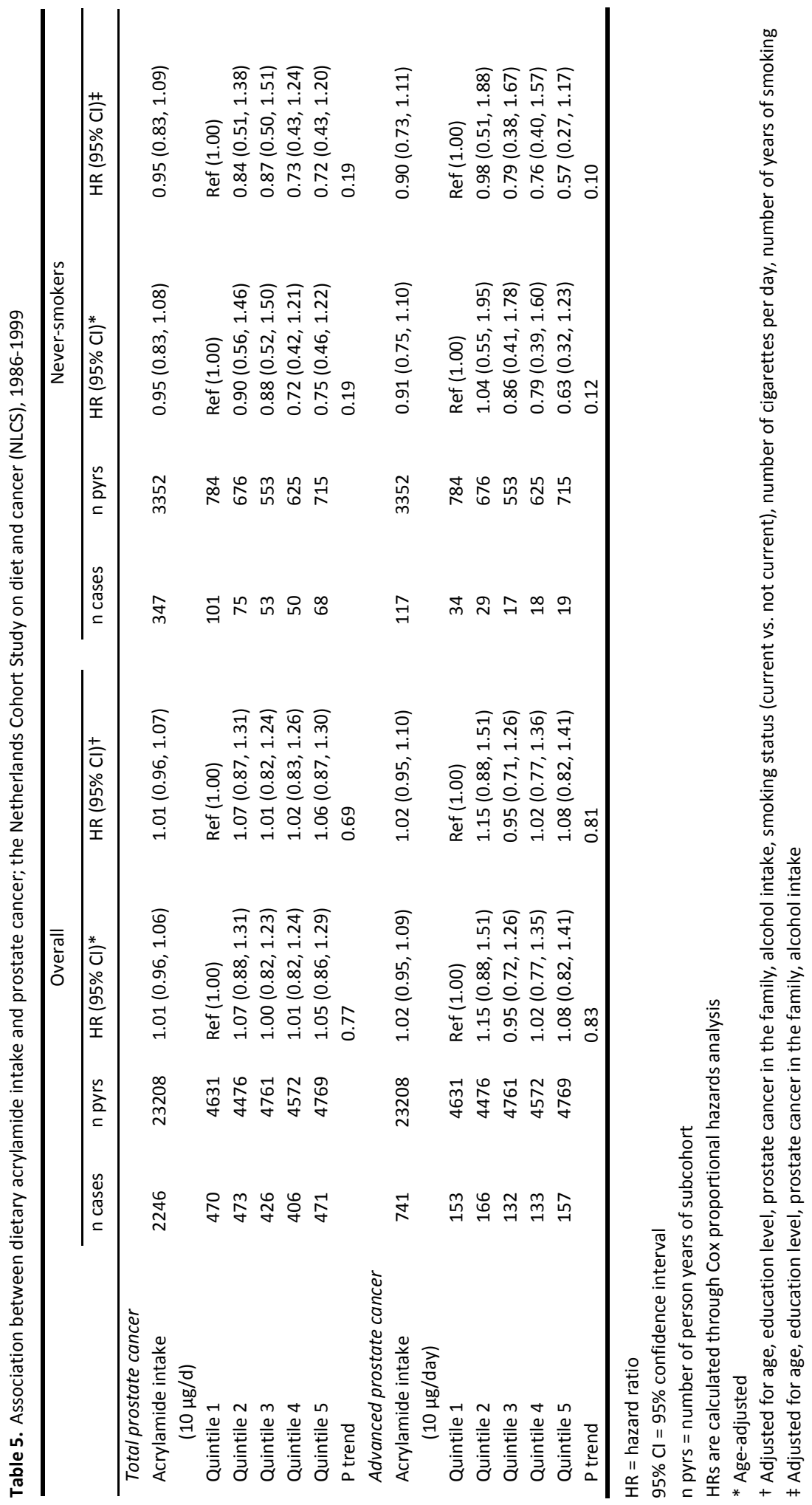




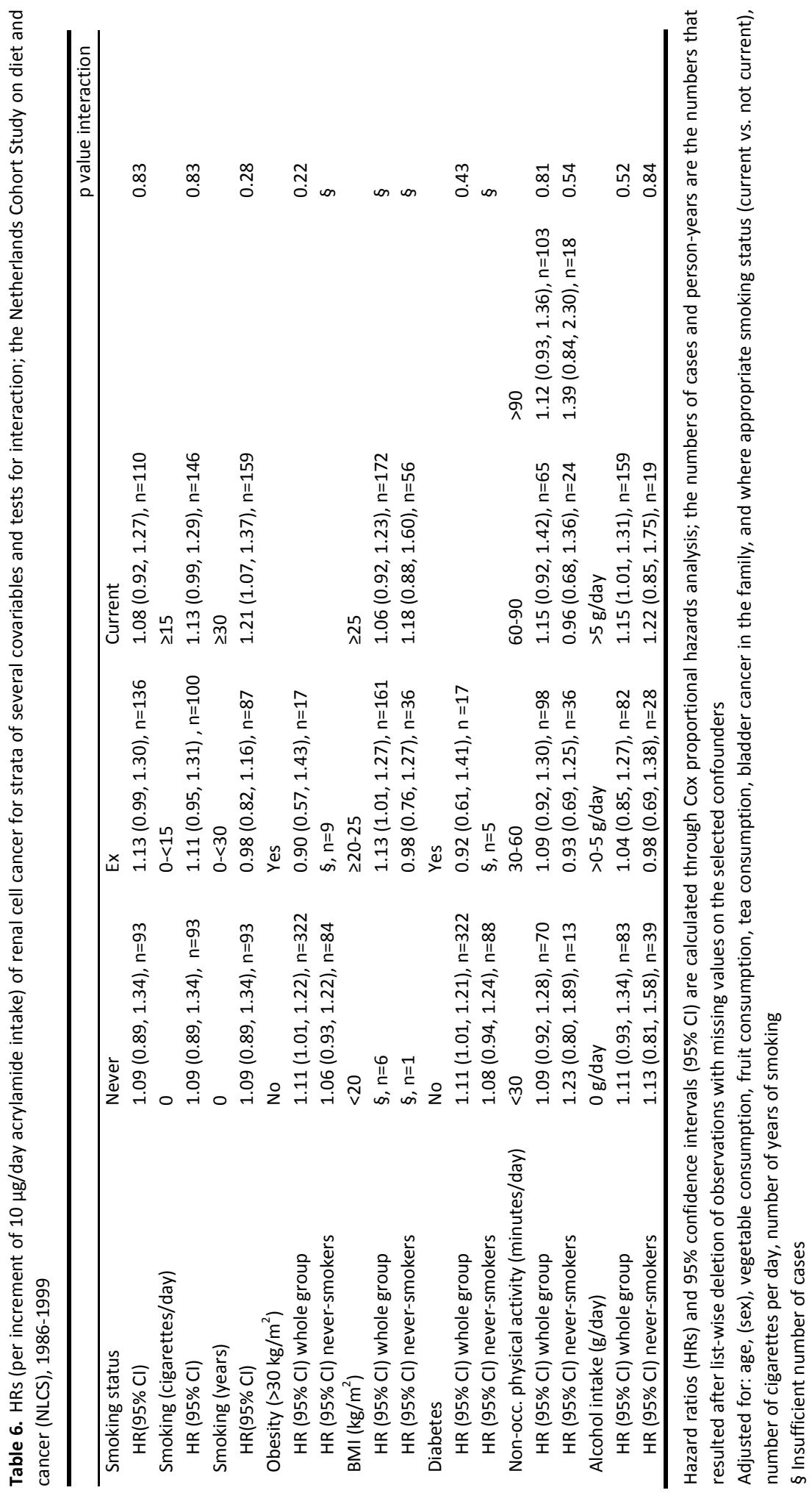




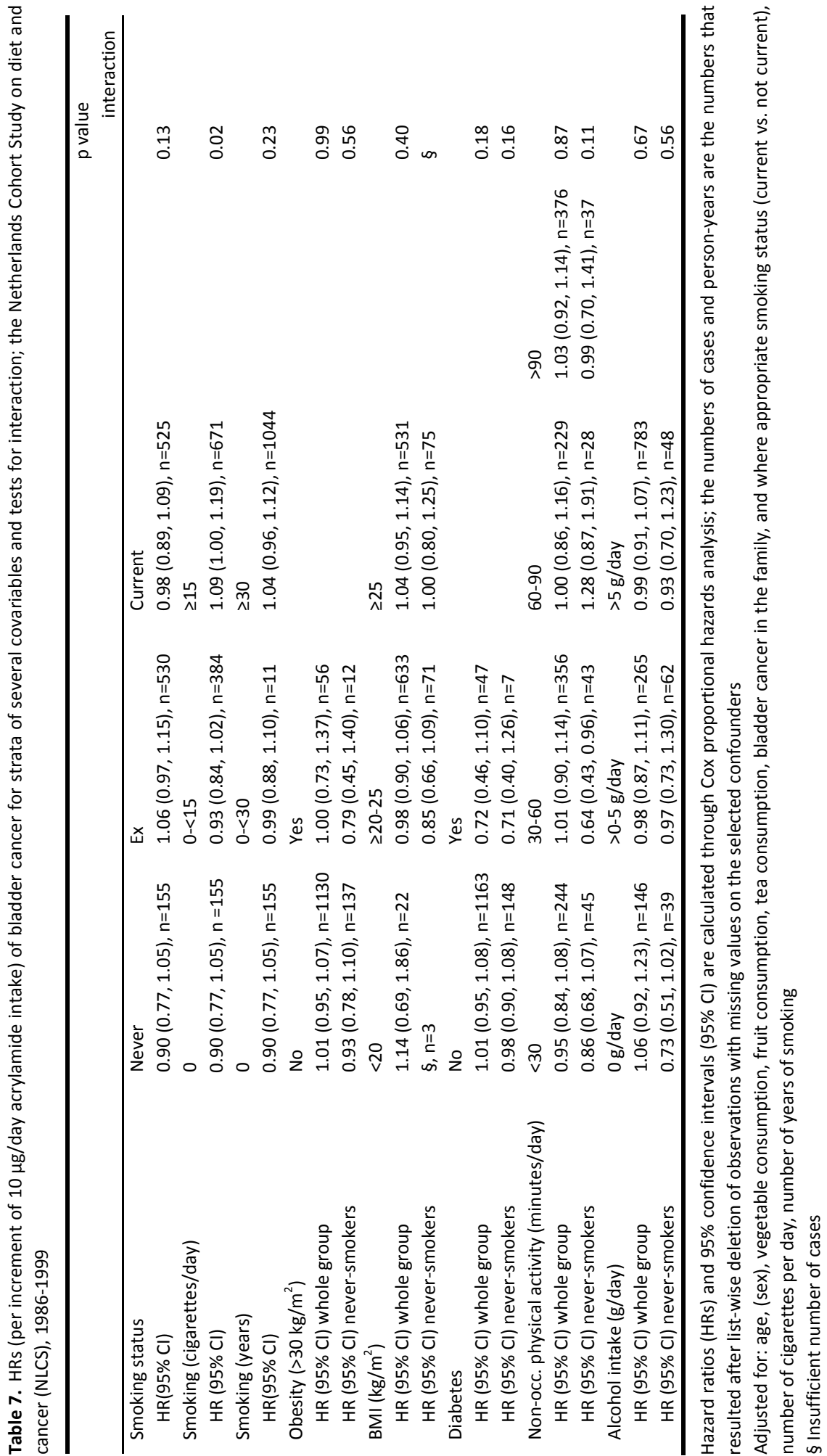


Dietary acrylamide intake and renal cell, bladder and prostate cancer risk

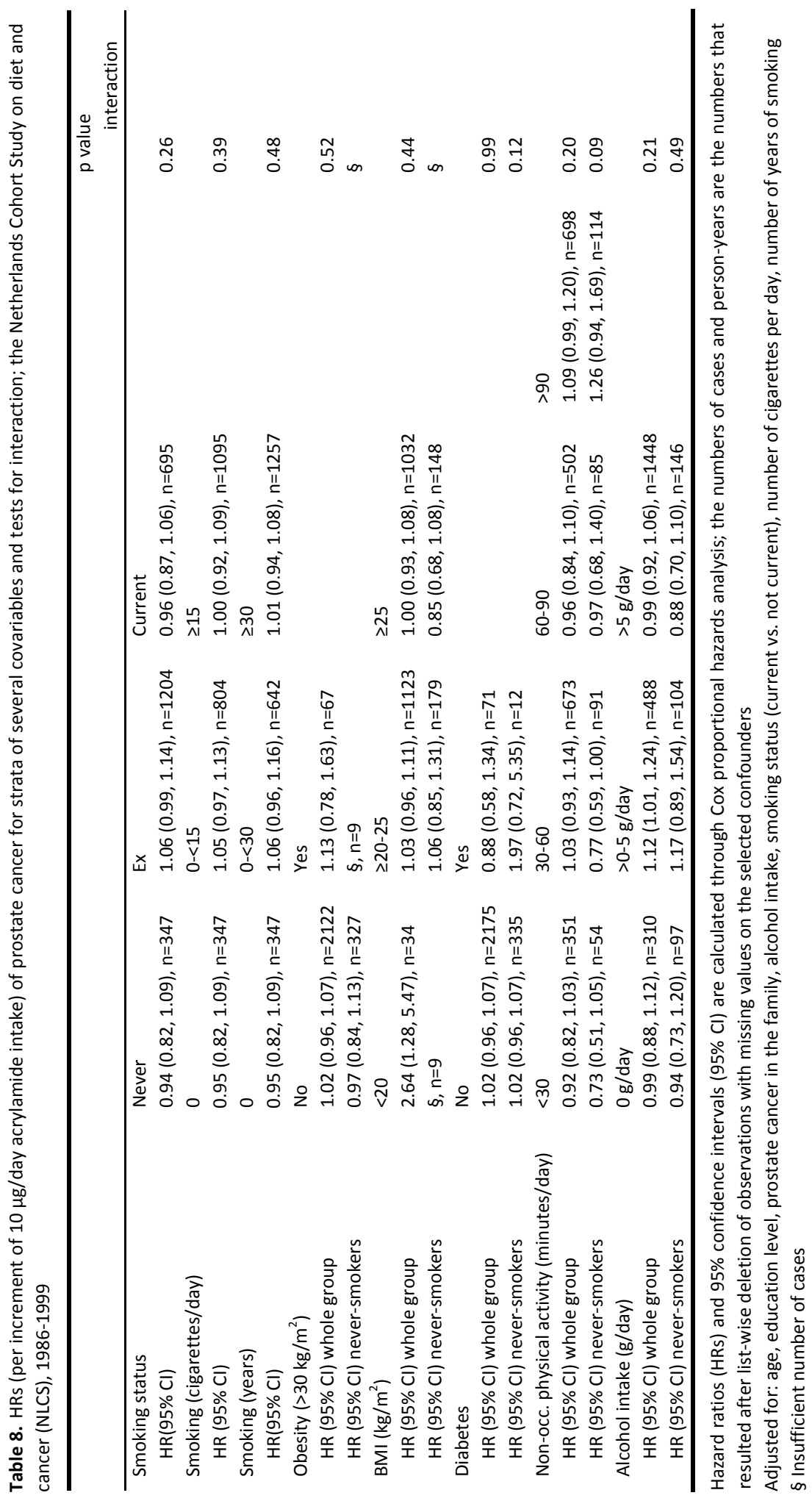


interaction suggest that none of the variables statisticically significantly modified the prostate cancer risk associated with acrylamide intake (Table 8).

\section{Discussion}

This prospective cohort study provides indications that dietary acrylamide intake may increase the risk of renal cell cancer.

In two case-control studies in Sweden, no association between acrylamide intake and renal cell cancer risk was observed $(18,19)$, but case-control studies may suffer from recall bias and selection bias, which might distort true associations. These types of biases are not relevant in the present prospective cohort study, which includes a nearly complete follow-up. In never or former smokers, there was no association between acrylamide and renal cell cancer risk, which may be explained by the higher risk in the subgroup with the longest duration of smoking (see Table 6). Thus, there may be a synergy between acrylamide intake and duration of smoking.

In the present study, acrylamide intake, overall, was not positively associated with a risk of bladder cancer, whether total or invasive. This finding is in line with the only study on this topic from Sweden. However, in that case-control study, the association was not evaluated separately for men and women. In the present study, there was a borderline statistically significant inverse trend but no clear linear dose-response relation for the association between acrylamide intake and bladder cancer risk in never-smokers; this trend appeared to be restricted to the female subgroup. On the basis of this study alone, we do not make a statement about whether there may be an inverse non-linear doseresponse relation between acrylamide intake and bladder cancer risk in women. It is too early to say whether the observed significantly decreased HRs in some quintiles of acrylamide intake represent a real biological effect or whether they are due to random fluctuations. More research on acrylamide intake and bladder cancer risk in women is needed. In contrast, there was a significant interaction between acrylamide intake and smoking in terms of the number of cigarettes smoked per day.

We found no positive association between acrylamide intake and prostate cancer risk. We did, however, observe an inverse linear trend in never-smokers but it was not statistically significant. In the only other epidemiological study on dietary acrylamide intake and prostate cancer risk so far, no association was observed (17), but in that case-control study, no subgroup analyses were done for never-smokers.

The relative risks of renal cell cancer associated with dietary acrylamide intake that we have observed in the present study are considerably higher than the relative risks that would be expected on the basis of linear extrapolation of the results of a cancer incidence study in rodents (40), using the tissue that was most sensitive to carcinogenesis. In rodents, no tumours were observed in the kidney upon acrylamide administration (7-10). However, the results of extrapolating the high acrylamide dosages that were administered to animals to the low dosages that humans are exposed to through food are uncertain. The activity or capacity of enzymes in the metabolism of acrylamide may be either in- 
creased or decreased at low doses, and humans may differ from animals in their ability to otherwise detoxify or eliminate acrylamide or glycidamide. The genotoxic action of glycidamide $(35,41,42)$ is currently adopted as the mechanism of carcinogenic action in acrylamide cancer risk assessments. In a previous study, we observed positive associations between acrylamide intake and both endometrial and ovarian cancer risk (22), which might indicate that disturbance of hormonal balances may also be a mechanism of acrylamide carcinogenesis. Rodents may not be a good (quantitative) model for such hormonal effects.

The associations between acrylamide intake and renal cell, bladder, and prostate cancer risk that were observed in this study may be a reflection of the potential hormonal influences of acrylamide. A recent in vitro study on glycidamide-induced gene expression in human breast and colon cancer cells adds to this theory by showing up-regulation of genes that catalyse the conversion of inactive androgen and estrogen precursors to active forms, such as testosterone, and $17 \beta$-estradiol (43). Renal cell cancer risk has been positively associated with estrogens in both animals and humans $(44,45)$. In prospective cohort studies, indications were found for an inverse association between estrogen and bladder cancer risk, in the form of inverse associations between age at menopause and bladder cancer risk and between lifetime years of ovulation and bladder cancer risk (46). In addition, both rodent and human bladder cells express estrogen receptors, and estrogens inhibit bladder carcinogenesis in rodents (46). If acrylamide were to increase the concentrations of free estrogen in the body, that could explain both its positive association with endometrial, ovarian cancer, and renal cell cancer risk and its inverse association with bladder cancer risk in women. Furthermore, acrylamide has been shown to decrease concentrations of plasma testosterone in rats upon acrylamide injection into the rat brain (47). This finding may explain the decrease in HRs for prostate cancer in never-smokers across the quintiles of acrylamide intake that was observed in the present study; the question of whether a high concentration of testosterone is indeed a risk factor for prostate cancer, is, however, still under debate (48). In a prospective cohort study, in which the risk ratios for several hormones were mutually adjusted for each other and for sex hormone-binding globulin, testosterone was positively associated and estrogens were inversely associated with the risk of prostate cancer (49).

This study has some limitations. FFQs have limitations, as discussed elsewhere (50), but they are the only feasible way to assess dietary intake over a long period in large-scale epidemiological studies. Acrylamide adducts to haemoglobin can be used as a biomarker of exposure (31), but they represent the exposure during the preceding three months only. Furthermore, biomarkers are not specific with regard to the source of acrylamide. Thus, in biomarker studies of dietary acrylamide exposure, strict control for smoking and passive smoking is of the utmost importance. Moreover, the costs of using biomarkers limit the size of the population that can be used in a study. The NLCS FFQ has proved to be both valid (27) and reproducible (28), also with respect to nutrients that correlate with acrylamide, such as carbohydrates and fibre. The acrylamide intake assessment is an important asset of the present study, together with its large study size and prospective nature, the latter of which excludes selection and recall bias. We used acrylamide concen- 
trations in foods from the Dutch market only, and we specifically sampled and analysed foods that were relevant for the NLCS population.

Within the validation study that we described in the Methods section, visual inspection of the scatter plot and the Bland-Altman plot showed that most of the estimated amounts were higher than the corresponding measured amounts. This implies that the use of mean values of acrylamide concentrations in foods to estimate dietary acrylamide intake on a continuous scale probably leads to some underestimation of the true acrylamide-associated risk.

It has to be borne in mind that the variation in acrylamide intake in our study was to a large extent due to Dutch spiced cake, which resembles gingerbread of the cake-like, noncrusty, type. Dutch spiced cake does not necessarily contain ginger, but it contains spices and sometimes fruit. Concentrations of acrylamide in Dutch spiced cake (mean: $1018 \mathrm{ppb}$ ) are much higher than those in plain cake (mean: $<30 \mathrm{ppb}$ ). Because of the addition of glucose and fructose syrup, Dutch spiced cake contains a greater amount of reducing sugars than do other cakes. In the presence of ammonium hydrogen carbonate, which is often used as a baking agent in spiced cake, the formation of acrylamide is further enhanced (51). There is no indication that the associations between acrylamide intake and renal cell, bladder, and prostate cancer risk should be attributed to other ingredients of spiced cake; the results did not change significantly after adjustment for spiced cake.

For the interaction analyses, we calculated many HRs in many small subgroups, which makes it likely that some of the observed significant $P$ values for interaction or HRs in subgroups were spurious. Therefore, they should be interpreted cautiously and their analysis should be repeated in other epidemiological studies.

Current populations, especially young people, consume larger amounts of potato crisps and French fries than did the NLCS population. Because of the high acrylamide concentrations of these foods, current dietary intake of acrylamide is assumed to be considerably higher $(29,31,52)$ than the intake in the present study. In contrast, industry and science are investigating ways to reduce acrylamide concentrations in food and have succeeded to some extent with respect to certain foods (53).

Although replication in other prospective studies is needed, the present study suggests that acrylamide is not neutral with regard to carcinogenesis. Depending on the tissue, acrylamide may have both carcinogenic and anticarcinogenic properties, which suggests that the genotoxic action of glycidamide, the epoxide metabolite of acrylamide, may not be the (only) pathway through which acrylamide exerts its effects. The results of the interaction analyses between acrylamide and the CYP2E1-modifying variables also do not clearly point toward a glycidamide-mediated genotoxic effect, because they do not modify, or at least do not consistently modify, the association between acrylamide intake and renal cell, bladder, and prostate cancer risk.

We encourage other researchers to prospectively investigate the association between dietary acrylamide intake and renal cell, bladder, and prostate cancer risk; to perform subgroup analyses for non-smokers; and to study interactions with factors that modify CYP2E1 activity. In the mean time, the indications of a positive association between acrylamide intake and endometrial, ovarian, and renal cell cancer risk should be an incentive for food industries to continue striving to lower acrylamide concentrations in foods. 


\section{References}

1. IARC. Monographs on the evaluation of carcinogen risk to humans: some industrial chemicals. Lyon: International Agency for Research on Cancer, 1994.

2. Tareke E, Rydberg P, Karlsson P, Eriksson S, Tornqvist M. Analysis of acrylamide, a carcinogen formed in heated foodstuffs. J Agric Food Chem 2002;50:4998-5006.

3. Summary and conclusions of the sixty-fourth meeting of the Joint FAO/WHO Expert Committee on Food Additives (JECFA). JECFA, 2005.

4. Mottram DS, Wedzicha BL, Dodson AT. Acrylamide is formed in the Maillard reaction. Nature 2002;419:4489.

5. Stadler RH, Blank I, Varga N, et al. Acrylamide from Maillard reaction products. Nature 2002;419:449-50.

6. Besaratinia A, Pfeifer GP. A review of mechanisms of acrylamide carcinogenicity. Carcinogenesis 2007;28:519-28.

7. Johnson KA, Gorzinski SJ, Bodner KM, et al. Chronic toxicity and oncogenicity study on acrylamide incorporated in the drinking water of Fischer 344 rats. Toxicol Appl Pharmacol 1986;85:154-68.

8. Bull RJ, Robinson M, Laurie RD, et al. Carcinogenic effects of acrylamide in Sencar and A/J mice. Cancer Res 1984;44:107-11.

9. Bull RJ, Robinson M, Stober JA. Carcinogenic activity of acrylamide in the skin and lung of Swiss-ICR mice. Cancer Lett 1984;24:209-12.

10. Friedman MA, Dulak LH, Stedham MA. A lifetime oncogenicity study in rats with acrylamide. Fundam Appl Toxicol 1995;27:95-105.

11. Marsh GM, Lucas L, Youk AO, Schall LC. Mortality patterns among workers exposed to acrylamide: 1994 follow up. Occup Environ Med 1999;56:181-90.

12. Schulz MR, Hertz-Picciotto I, van Wijngaarden E, Hernandez JC, Ball LM. Dose-response relation between acrylamide and pancreatic cancer. Occup Environ Med 2001;58:609.

13. Sobel W, Bond GG, Parsons TW, Brenner FE. Acrylamide cohort mortality study. Br J Ind Med 1986;43:7858.

14. Collins JJ, Swaen GM, Marsh GM, Utidjian HM, Caporossi JC, Lucas LJ. Mortality patterns among workers exposed to acrylamide. J Occup Med 1989;31:614-7.

15. Swaen GM, Haidar S, Burns CJ, et al. Mortality study update of Acrylamide workers. Occup Environ Med 2007;64:396-401.

16. Marsh GM, Youk AO, Buchanich JM, Kant IJ, Swaen G. Mortality patterns among workers exposed to acrylamide: updated follow up. J Occup Environ Med 2007;49:82-95.

17. Pelucchi C, Galeone C, Levi F, et al. Dietary acrylamide and human cancer. Int J Cancer 2006;118:467-71.

18. Mucci LA, Dickman PW, Steineck G, Adami HO, Augustsson K. Dietary acrylamide and cancer of the large bowel, kidney, and bladder: absence of an association in a population-based study in Sweden. Br J Cancer 2003;88:84-9.

19. Mucci LA, Lindblad P, Steineck G, Adami HO. Dietary acrylamide and risk of renal cell cancer. Int J Cancer 2004;109:774-6.

20. Mucci LA, Adami HO, Wolk A. Prospective study of dietary acrylamide and risk of colorectal cancer among women. Int J Cancer 2006;118:169-73.

21. Mucci LA, Sandin S, Balter K, Adami HO, Magnusson C, Weiderpass E. Acrylamide intake and breast cancer risk in Swedish women. Jama 2005;293:1326-7.

22. Hogervorst JG, Schouten LJ, Konings EJ, Goldbohm RA, van den Brandt PA. A Prospective Study of Dietary Acrylamide Intake and the Risk of Endometrial, Ovarian and Breast Cancer. Cancer Epidemiol Biomarkers Prev 2007;16:2304-13.

23. Friedman M. Chemistry, biochemistry, and safety of acrylamide. A review. J Agric Food Chem 2003;51:450426.

24. van den Brandt PA, Goldbohm RA, van 't Veer P, Volovics A, Hermus RJ, Sturmans F. A large-scale prospective cohort study on diet and cancer in The Netherlands. J Clin Epidemiol 1990;43:285-95.

25. Goldbohm RA, van den Brandt PA, Dorant E. Estimation of the coverage of Dutch municipalities by cancer registries and PALGA based on hospital discharge data. Tijdschr Soc Gezondheidsz 1994;72:80-84.

26. van den Brandt PA, Schouten LJ, Goldbohm RA, Dorant E, Hunen PM. Development of a record linkage protocol for use in the Dutch Cancer Registry for Epidemiological Research. Int J Epidemiol 1990;19:553-8.

27. Goldbohm RA, van den Brandt PA, Brants HA, et al. Validation of a dietary questionnaire used in a largescale prospective cohort study on diet and cancer. Eur J Clin Nutr 1994;48:253-65. 
28. Goldbohm RA, van ' $t$ Veer $P$, van den Brandt PA, et al. Reproducibility of a food frequency questionnaire and stability of dietary habits determined from five annually repeated measurements. Eur J Clin Nutr 1995;49:420-9.

29. Konings EJ, Baars AJ, van Klaveren JD, et al. Acrylamide exposure from foods of the Dutch population and an assessment of the consequent risks. Food Chem Toxicol 2003;41:1569-79.

30. Database of the European Commission Institute for Reference Materials and Measurements (IRMM) (accessed August 3, 2006, at http://www.irmm.jrc.be/html/activities/acrylamide/database.htm)

31. Dybing $E$, Farmer PB, Andersen $M$, et al. Human exposure and internal dose assessments of acrylamide in food. Food Chem Toxicol 2005;43:365-410.

32. Schottenfeld D, Fraumeni JF, Jr. Cancer Epidemiology and Prevention. third ed. New York City: Oxford University Press, 2006.

33. Schettgen T, Rossbach B, Kutting B, Letzel S, Drexler H, Angerer J. Determination of haemoglobin adducts of acrylamide and glycidamide in smoking and non-smoking persons of the general population. Int J Hyg Environ Health 2004;207:531-9.

34. Bergmark E. Hemoglobin adducts of acrylamide and acrylonitrile in laboratory workers, smokers and nonsmokers. Chem Res Toxicol 1997;10:78-84.

35. Ghanayem BI, Witt KL, Kissling GE, Tice RR, Recio L. Absence of acrylamide-induced genotoxicity in CYP2E1null mice: evidence consistent with a glycidamide-mediated effect. Mutat Res 2005;578:284-97.

36. Lamy E, Volkel Y, Roos PH, Kassie F, Mersch-Sundermann V. Ethanol enhanced the genotoxicity of acrylamide in human, metabolically competent HepG2 cells by CYP2E1 induction and glutathione depletion. Int J Hyg Environ Health 2008;211:74-81.

37. Ardies CM, Smith TJ, Kim S, Yang CS. Induction of 4-(methylnitrosamino)-1-(3-pyridyl)-1-butanone (NNK) activation in rat lung microsomes by chronic ethanol consumption and repeated running exercise. Cancer Lett 1996;103:209-18.

38. Howard LA, Micu AL, Sellers EM, Tyndale RF. Low doses of nicotine and ethanol induce CYP2E1 and chlorzoxazone metabolism in rat liver. J Pharmacol Exp Ther 2001;299:542-50.

39. Wang Z, Hall SD, Maya JF, Li L, Asghar A, Gorski JC. Diabetes mellitus increases the in vivo activity of cytochrome P450 2E1 in humans. Br J Clin Pharmacol 2003;55:77-85.

40. Dybing E, Sanner T. Risk assessment of acrylamide in foods. Toxicol Sci 2003;75:7-15.

41. Gamboa da Costa G, Churchwell MI, Hamilton LP, et al. DNA adduct formation from acrylamide via conversion to glycidamide in adult and neonatal mice. Chem Res Toxicol 2003;16:1328-37.

42. Doerge DR, da Costa GG, McDaniel LP, Churchwell MI, Twaddle NC, Beland FA. DNA adducts derived from administration of acrylamide and glycidamide to mice and rats. Mutat Res 2005;580:131-41.

43. Clement FC, Dip R, Naegeli H. Expression profile of human cells in culture exposed to glycidamide, a reactive metabolite of the heat-induced food carcinogen acrylamide. Toxicology 2007;240:111-24.

44. Tanaka Y, Sasaki M, Kaneuchi M, Fujimoto S, Dahiya R. Estrogen receptor alpha polymorphisms and renal cell carcinoma--a possible risk. Mol Cell Endocrinol 2003;202:109-16.

45. Nicodemus KK, Sweeney C, Folsom AR. Evaluation of dietary, medical and lifestyle risk factors for incident kidney cancer in postmenopausal women. Int J Cancer 2004;108:115-21.

46. Prizment $\mathrm{AE}$, Anderson $\mathrm{KE}$, Harlow BL, Folsom AR. Reproductive risk factors for incident bladder cancer: Iowa Women's Health Study. Int J Cancer 2006;120:1093-8.

47. Ali SF, Hong JS, Wilson WE, Uphouse LL, Bondy SC. Effect of acrylamide on neurotransmitter metabolism and neuropeptide levels in several brain regions and upon circulating hormones. Arch Toxicol 1983;52:3543.

48. Platz EA, Giovannucci E. The epidemiology of sex steroid hormones and their signaling and metabolic pathways in the etiology of prostate cancer. J Steroid Biochem Mol Biol 2004;92:237-53.

49. Gann PH, Hennekens CH, Ma J, Longcope C, Stampfer MJ. Prospective study of sex hormone levels and risk of prostate cancer. J Natl Cancer Inst 1996;88:1118-26.

50. Konings EJ, Hogervorst JGF, Schouten $\amalg$, van den Brandt PA. Assessing exposure levels of acrylamide. In: Skog K, Alexander J, eds. Acrylamide and other hazardous compounds in heat-treated foods. Cambridge: Woodhead Publishing Limited, 2006:214-225.

51. Amrein TM, Andres L, Manzardo GG, Amado R. Investigations on the promoting effect of ammonium hydrogencarbonate on the formation of acrylamide in model systems. J Agric Food Chem 2006;54:1025361.

52. Matthys C, Bilau M, Govaert Y, Moons E, De Henauw S, Willems JL. Risk assessment of dietary acrylamide intake in Flemish adolescents. Food Chem Toxicol 2005;43:271-8. 
53. Acrylamide Status Report December 2004: a summary of the efforts and progress achieved to date by the European food and drink industry (CIAA) in lowering levels of acrylamide in food. Bruxelles: CIAA, 2004. 



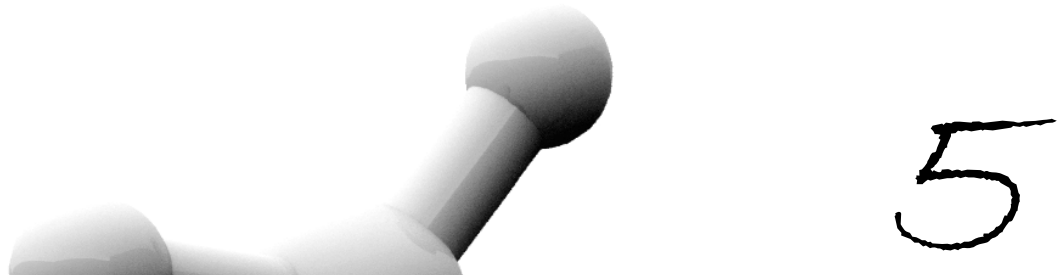

\section{Dietary acrylamide intake is not} associated with gastrointestinal cancer risk

Janneke G.F. Hogervorst Leo J. Schouten

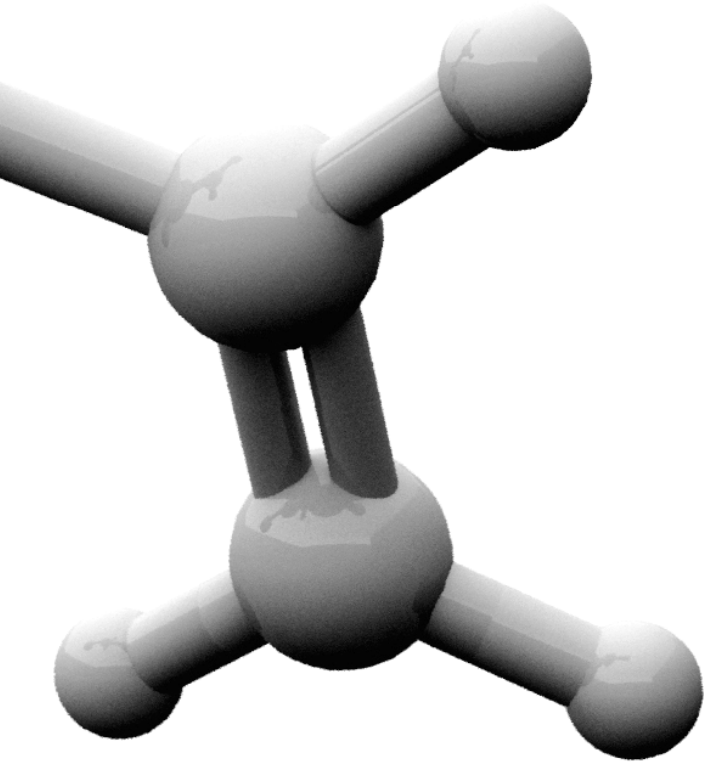

Erik J.M. Konings

R. Alexandra Goldbohm

Piet A. van den Brandt 


\begin{abstract}
Acrylamide is a probable human carcinogen that was detected in several heat-treated foods, such as French fries and crisps, in 2002. Prospective studies are needed on acrylamide and human cancer risk. We prospectively investigated the association between acrylamide and gastrointestinal cancer risk. In 1986, 120852 men and women (aged 55-69 y) were included in the Netherlands Cohort Study on diet and cancer. At baseline, a random subcohort of 5000 participants was selected for a case-cohort approach. Acrylamide intake was assessed with an FFQ at baseline and was based on acrylamide analyses in relevant Dutch foods. After 13.3 y of follow-up, 2190, 563, 349, and 216 cases of colorectal, gastric, pancreatic, and oesophageal cancer, respectively, were available for analysis. The mean daily acrylamide intake of the subcohort was 21.8 (SD 12.1) $\mu \mathrm{g}$. A $10 \mu \mathrm{g} / \mathrm{d}$ increment of acrylamide intake was associated with multivariable-adjusted Cox proportional hazard ratios (HRs) $(95 \% \mathrm{Cl}$ ) of 1.00 (0.96-1.06), 1.02 (0.94-1.10), 1.06 (0.96-1.17), and 0.96 (0.85-1.09) for colorectal, gastric, pancreatic, and oesophageal cancer, respectively. For former or never-smokers, the corresponding HRs were: 1.03 (0.94-1.12), 1.09 (0.98-1.22), 1.07 (0.93-1.24), and 0.92 (0.76-1.11). There were some significantly increased risks within subgroups stratified by obesity, non-occupational physical activity and age, factors that were $a$ priori selected based on their capacity to modify cytochrome P4502E1 activity. Overall, acrylamide intake was not associated with colorectal, gastric, pancreatic, and oesophageal cancer risk, but some subgroups deserve further attention.
\end{abstract}




\section{Introduction}

In 2002, acrylamide was detected at high concentrations in heat-processed carbohydraterich foods, such as French fries and potato crisps, and coffee (1). It is formed in Maillard browning reactions in which amino acids, particularly asparagine, react with reducing sugars at temperatures $>120^{\circ} \mathrm{C}(2,3)$.

Recently, in epidemiological studies, positive associations have been observed for endometrial and ovarian cancer (4), renal cell cancer (5), and postmenopausal estrogen receptor-positive breast cancer risk (6). In a retrospective cohort study from 1986 on occupational acrylamide exposure, a positive association was observed between cumulative acrylamide exposure and pancreatic cancer risk $(7,8)$. These findings, combined with the fact that acrylamide is present at high concentrations in many everyday foods, stress the need for more prospective studies on the association between dietary acrylamide and cancer risk.

In 1994, the International Agency for Research on Cancer classified the industrial chemical acrylamide as a probable human carcinogen, based on its carcinogenic action in rodents (9). Animal studies have shown positive dose-response relationships between acrylamide exposure and cancer at multiple sites (10), e.g., oral tissues, thyroid gland, mammary gland, lung, and skin. Both genotoxic and non-genotoxic pathways have been suggested for the carcinogenic effect of acrylamide. Acrylamide itself and its epoxide metabolite glycidamide, which is generated by cytochrome P4502E1, are clastogenic and glycidamide forms DNA adducts. As for possible non-genotoxic pathways, acrylamide may influence the redox status of cells and thus gene transcription or it may interfere with DNA repair or hormonal balances (10).

Epidemiological studies on occupational acrylamide exposure have been negative, apart from the positive association with pancreatic cancer risk mentioned above $(7,8,11$ 14). Despite the recommendation of the WHO to perform epidemiological studies on dietary acrylamide and cancer risk links (15), only a few case-control studies (16-18) and prospective cohort studies $(4-6,19,20)$, have been published up to now. Most of these studies rendered no indications for a positive association, except for the three studies mentioned previously (4-6).

Because the acrylamide molecule is small and hydrophilic, it reaches every organ and virtually every tissue in the body (21). For this reason, theoretically all tissues are targets for carcinogenesis. When acrylamide is taken in orally, the gastrointestinal tract is exposed to considerable amounts of this substance. Gastric and pancreatic cancers have not been studied before, to our knowledge, in epidemiological studies on dietary acrylamide intake. Pancreatic cancer had our particular interest, because it was associated with acrylamide exposure in one study on occupational acrylamide exposure. 


\section{Subjects and Methods}

\section{Study participants}

The Netherlands Cohort Study on diet and cancer (NLCS) started in September 1986 with the inclusion of 58279 men and 62573 women aged 55-69 y sampled from Dutch municipal registries (22). At baseline, the participants completed a self-administered questionnaire on diet and other cancer risk factors. The case-cohort approach was used; cases were enumerated for the entire cohort, whereas the accumulated person-years for the total cohort were estimated from a subcohort of 5000 participants randomly sampled from the full cohort at baseline. Since the start of the study, vital status information was obtained from the subcohort at regular time intervals. Incident cases in the total cohort were detected by annual record linkages to the regional cancer registries and the Netherlands Pathology Registry. The completeness of cancer follow-up was assessed to be at least $96 \%$ (23), whereas the follow-up of the subcohort at the end of follow-up was nearly $100 \%$ complete (only two male subcohort members were lost to follow-up). The study was approved by the Medical Ethics Committees of the University Hospital Maastricht and TNO Nutrition in February 1985 and July 1986, respectively. Further details on the design of the study and methods of follow-up are presented elsewhere (22, 24-26).

The analyses are based on 13.3 y of follow-up (September 1986-January 2000). There were 2740 colorectal (1818 colon (ICD-O-3 C18), 278 rectosigmoid (ICD-O-3 C19), and 644 rectal (ICD-O-3 C20) cancer cases). Among the 681 gastric cancer cases, 180 adenocarcinomas of the cardia (ICD-O-3 C16.0), 284 adenocarcinomas of the distant stomach (ICD-O3 C16.1-C16.5), and 217 adenocarcinomas of the stomach with an unspecified localisation (ICD-0-3 C16.6-C16.9) were detected. Of the 445 pancreatic cancer cases (ICD-O-3 C25, excluding C25.4), 289 cases were microscopically verified. Among the 267 oesophageal cancer cases (ICD-O-3 C15), there were 142 adenocarcinomas (M8140-8141, 8190-8231, $8260-8263,8310,8430,8480-8490,8560,8570-8572)$, and 108 squamous cell carcinomas (M8050-8076).

Cases and subcohort members were excluded from analysis if they had cancer (other than skin cancer) at baseline and if their dietary data were incomplete or inconsistent (25), see Figure 1.

\section{Acrylamide intake assessment}

The 150-item FFQ queried the habitual intake of foods during the year preceding baseline (25). The acrylamide intake was estimated from the mean acrylamide level of food items and the frequency of consumption and portion size of foods. To be representative of our cohort, we used data on acrylamide concentrations in foods on the Dutch market only. Further details of the intake assessment, including concentrations of acrylamide in foods, are presented elsewhere (4). 


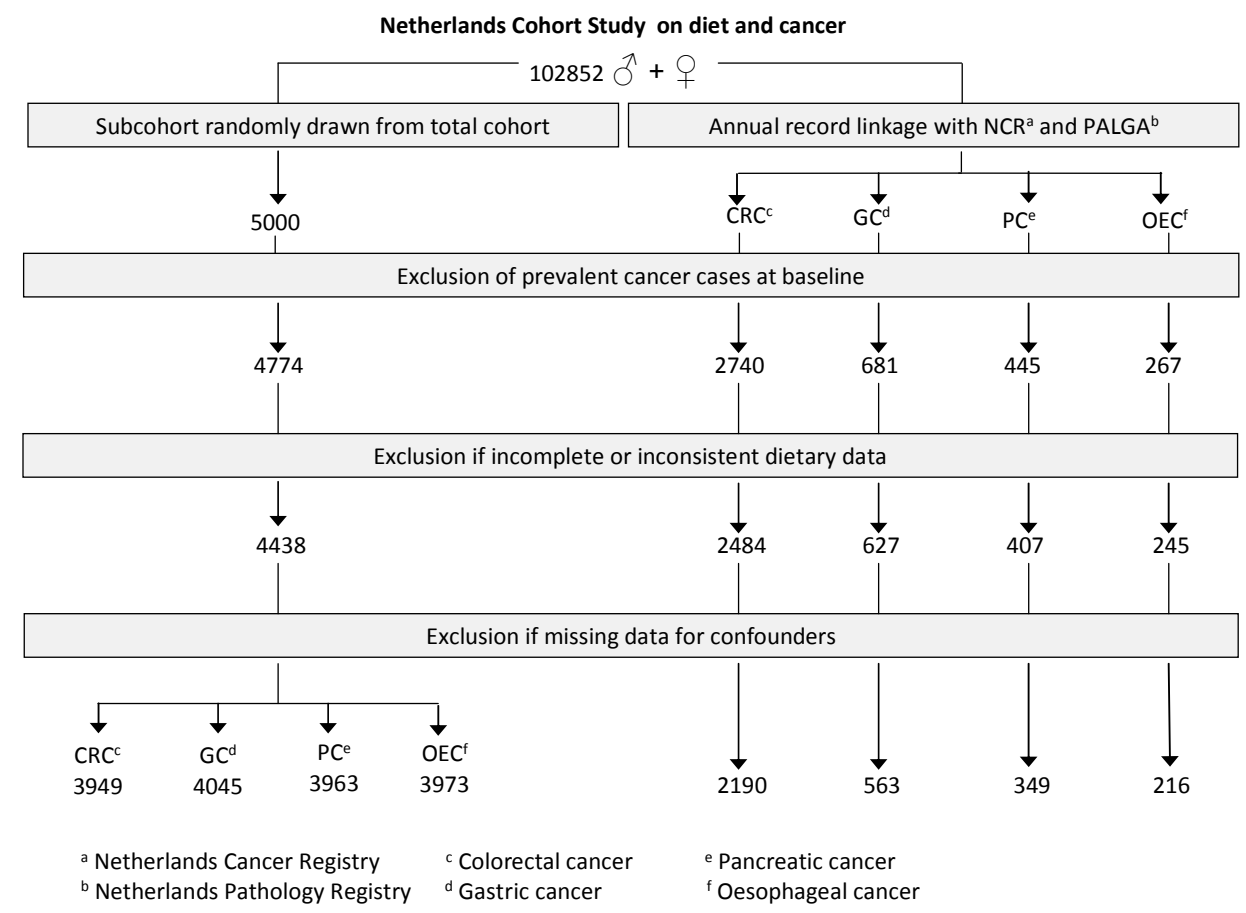

Figure 1. Flow diagram of subcohort members and cases on whom the analyses were based

\section{Statistical analysis}

Some confounders were chosen a priori and some were included in the models only if they changed age- and sex-adjusted hazard ratios (HRs) of acrylamide (expressed as the interval between the $10^{\text {th }}$ and $90^{\text {th }}$ percentile of intake of the subcohort: $27 \mu \mathrm{g}$ acrylamide/d) by more than $10 \%$ (Table 1). Smoking status, quantity and duration were always included in the models, because cigarette smoke is an important acrylamide source. Smokers have been shown to have on average four times higher levels of acrylamidehaemoglobin adducts, which is a marker of internal dose of acrylamide, than non-smokers $(27,28)$. For this reason, subgroup analyses were done for never-smokers. For gastric, pancreatic and oesophageal cancer, the number of never-smokers was too small, and therefore it was decided to combine never-smokers and ex-smokers that quitted more than ten years before the start of the study.

Scaled Schoenfeld residuals were used to test the proportional hazards assumption. HRs were obtained through Cox proportional hazards regression with STATA software (package 9.2). Additional variance introduced by sampling a subcohort from the cohort was taken into account by estimation of standard errors using the robust Huber-White sandwich estimator. Tests for trend were done by fitting the median acrylamide intake per quintile as a continuous variable. To check for the influence of preclinical disease, the analyses were also done excluding the first two years of follow-up. Effect modification by other variables was tested using Wald $\chi^{2}$ tests. The variables that were tested for effect 
modification were selected based on their ability to modify the activity of cytochrome P4502E1 and are age, diabetes, obesity, smoking, alcohol consumption, and physical activity (29-32).

Table 1. Selection of confounders for the multivariable-adjusted Cox regression analyses

\begin{tabular}{|l|l|l|l|}
\hline & A priori selected based on literature & $\begin{array}{l}\text { Tested for change of } \\
\text { HR }\end{array}$ & $\begin{array}{l}\text { Selected based on }> \\
10 \% \text { change of HR }\end{array}$ \\
\hline $\begin{array}{l}\text { Colorectal } \\
\text { cancer }\end{array}$ & $\begin{array}{l}\text { Age, sex, body mass index, height, energy, fibre } \\
\text { and vitamin B6 intake, consumption of } \\
\text { vegetables, fruits, dairy, meat, and alcohol, non- } \\
\text { occupational physical activity, family history of } \\
\text { colorectal cancer }\end{array}$ & $\begin{array}{l}\text { Education level, tea } \\
\text { consumption }\end{array}$ & - \\
\hline $\begin{array}{l}\text { Gastric } \\
\text { cancer }\end{array}$ & $\begin{array}{l}\text { Age, sex, body mass index, energy intake, tea } \\
\text { consumption, vegetables, fruits intake, education } \\
\text { level, and family history of gastric cancer }\end{array}$ & $\begin{array}{l}\text { Total fluid intake, and } \\
\text { meat, fish and cheese } \\
\text { intake }\end{array}$ & Fish intake \\
\hline $\begin{array}{l}\text { Pancreatic } \\
\text { cancer }\end{array}$ & $\begin{array}{l}\text { Age, sex, body mass index, height, energy intake, } \\
\text { consumption of vegetables, fruits, and alcohol, } \\
\text { diabetes, family history of pancreatic cancer }\end{array}$ & $\begin{array}{l}\text { Physical activity, dairy, } \\
\text { meat and fish intake }\end{array}$ & - \\
\hline $\begin{array}{l}\text { Oesophageal } \\
\text { cancer }\end{array}$ & $\begin{array}{l}\text { Age, sex, body mass index, consumption of tea, } \\
\text { vegetables, fruits, dairy, and alcohol intake, } \\
\text { family history of oesophageal cancer }\end{array}$ & $\begin{array}{l}\text { Education level, energy } \\
\text { intake, meat intake }\end{array}$ & - \\
\hline
\end{tabular}

\section{Results}

The mean daily acrylamide intake of the subcohort was 21.8 (SD 12.1) $\mu$ g. The most important dietary source of acrylamide in the subcohort was coffee, which contributed on average $47 \%$ to the acrylamide intake, whereas Dutch spiced cake, cookies, French fries, and potato crisps contributed $15,13,8$, and $2 \%$, respectively (5). However, most of the variance of the acrylamide intake was explained by Dutch spiced cake (57\%), followed by coffee (15\%), French fries (14\%), potato crisps (5\%), and cookies (3\%) (5).

The characteristics of cases and subcohort are shown in Table 2. Cases were older at baseline than subcohort members. The BMI of gastric cardia cancer, microscopically verified pancreatic cancer, and oesophageal adenocarcinoma cases was higher than that of the subcohort but lower for squamous cell oesophageal cancer cases. Smoking was more prevalent among all cancer case groups (except colon cancer) than among subcohort members, and cases smoked more and for a longer period. For dietary factors, cases consumed more alcohol than the subcohort, especially squamous cell oesophageal cancer cases. Fish consumption was higher in the gastric and squamous cell oesophageal cancer cases than in the subcohort. Finally, gastric cardia cancer and oesophageal adenocarcinoma cases drank less tea than the subcohort did.

For the proportional hazards analysis, men and women are combined, because there was no significant effect modification by sex. 
Overall, there were no indications for a positive association between acrylamide intake and colorectal cancer risk (Table 3). When the analyses were restricted to neversmokers, there was a significantly increased HR in the third quintile for colon and rectal cancer combined and colon cancer alone. There was no linear dose-response relationship over the quintiles. For rectal cancer, the HRs for never-smokers were significantly increased in the second and fourth quintile, but again there was no linear dose-response relationship. The results did not change when the first two years of follow-up were excluded (results not shown).

Acrylamide intake was not associated with total gastric cancer, gastric cardia adenocarcinoma, or non-cardia gastric cancer risk overall (Table 4). When the analyses were restricted to non-smokers, there were some indications ( $p=0.26$ and 0.19 , respectively) for a positive linear trend, both for cardia and non-cardia cancer risk. However, these associations weakened further when the first two years of follow-up were excluded.

Acrylamide intake was not associated with pancreatic (Table 5) or oesophageal cancer risk (Table 6). The same applies to the group of non-smokers and when the first two years of follow-up were excluded.

Reanalysing the age- and sex adjusted associations between acrylamide and cancer risk by excluding only observations with missing values on age and sex did not lead to other conclusions than excluding persons with missing values on any of the covariables in the multivariable-adjusted models. Therefore, bias due to non-randomness of the missing values on the covariables is not likely.

There was no significant effect modification by any of the studied variables for colorectal or gastric cancer. For microscopically verified pancreatic cancer, there was significant effect modification by obesity, with obese people having an increased risk $(95 \% \mathrm{Cl})$ of $1.59(0.87-2.89, \mathrm{n}=14)$ per $10 \mu \mathrm{g} / \mathrm{d}$ increment of acrylamide intake ( $\mathrm{p}$ for effect modification $=0.04)$. There was also effect modification $(p=0.02)$ by obesity for oesophageal cancer, particularly adenocarcinoma $(p=0.03)$, with obese participants having a $55 \%$ (8$121 \%, \mathrm{n}=20)$, and $90 \%(15-214 \%, \mathrm{n}=14)$ increased risk, respectively, for every $10 \mu \mathrm{g} / \mathrm{d}$ increment of acrylamide intake. The acrylamide-associated cancer risk was significantly increased in the subgroup with the highest non-occupational physical activity, although the test for effect modification was never significant. This was observed for colon cancer in never-smokers and for pancreatic cancer. There were significantly decreased acrylamide-associated risks for non-cardia gastric cancer in non-smokers and for squamous cell oesophageal cancer in the group with the lowest non-occupational physical activity. Although there was no significant effect modification by age, the oldest participants had a significantly decreased risk of rectal cancer per $10 \mu \mathrm{g} / \mathrm{d}$ acrylamide intake, whereas the youngest participants had a significantly increased acrylamide-associated pancreatic cancer risk. 


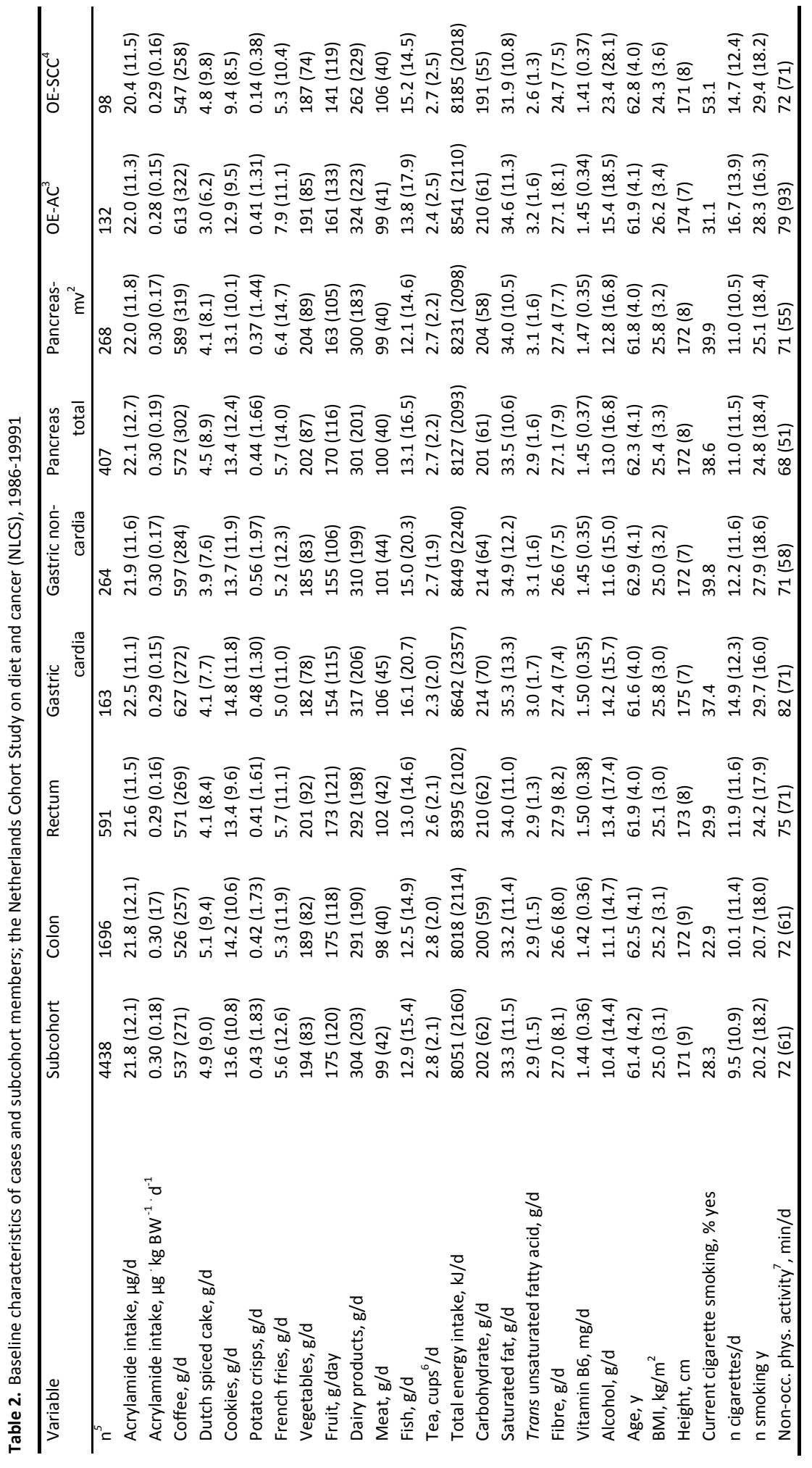




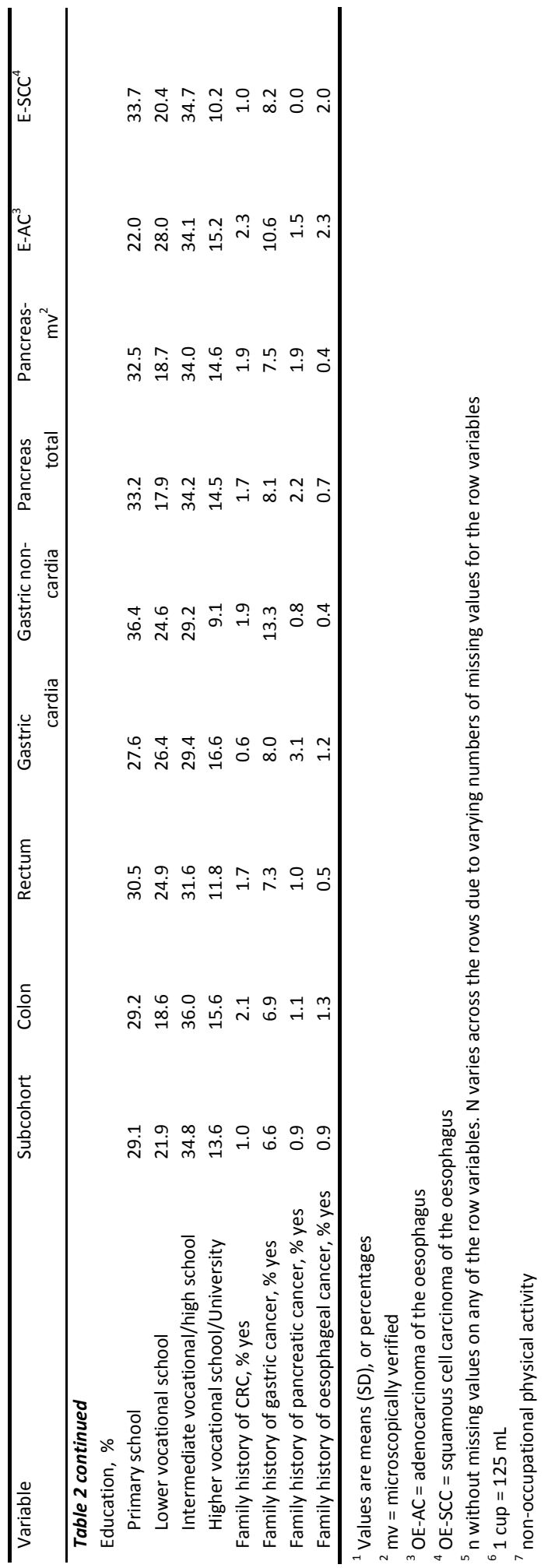




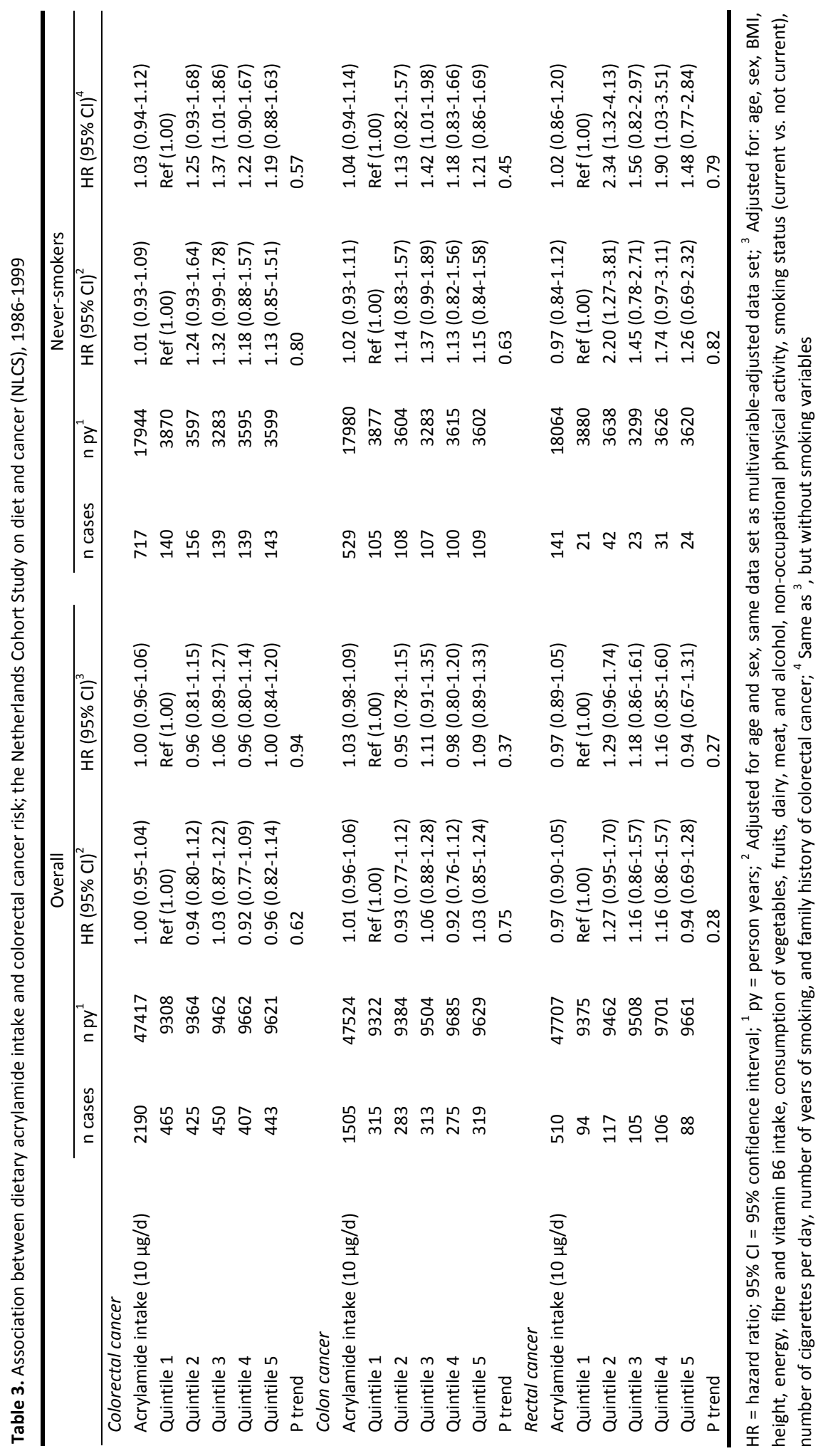




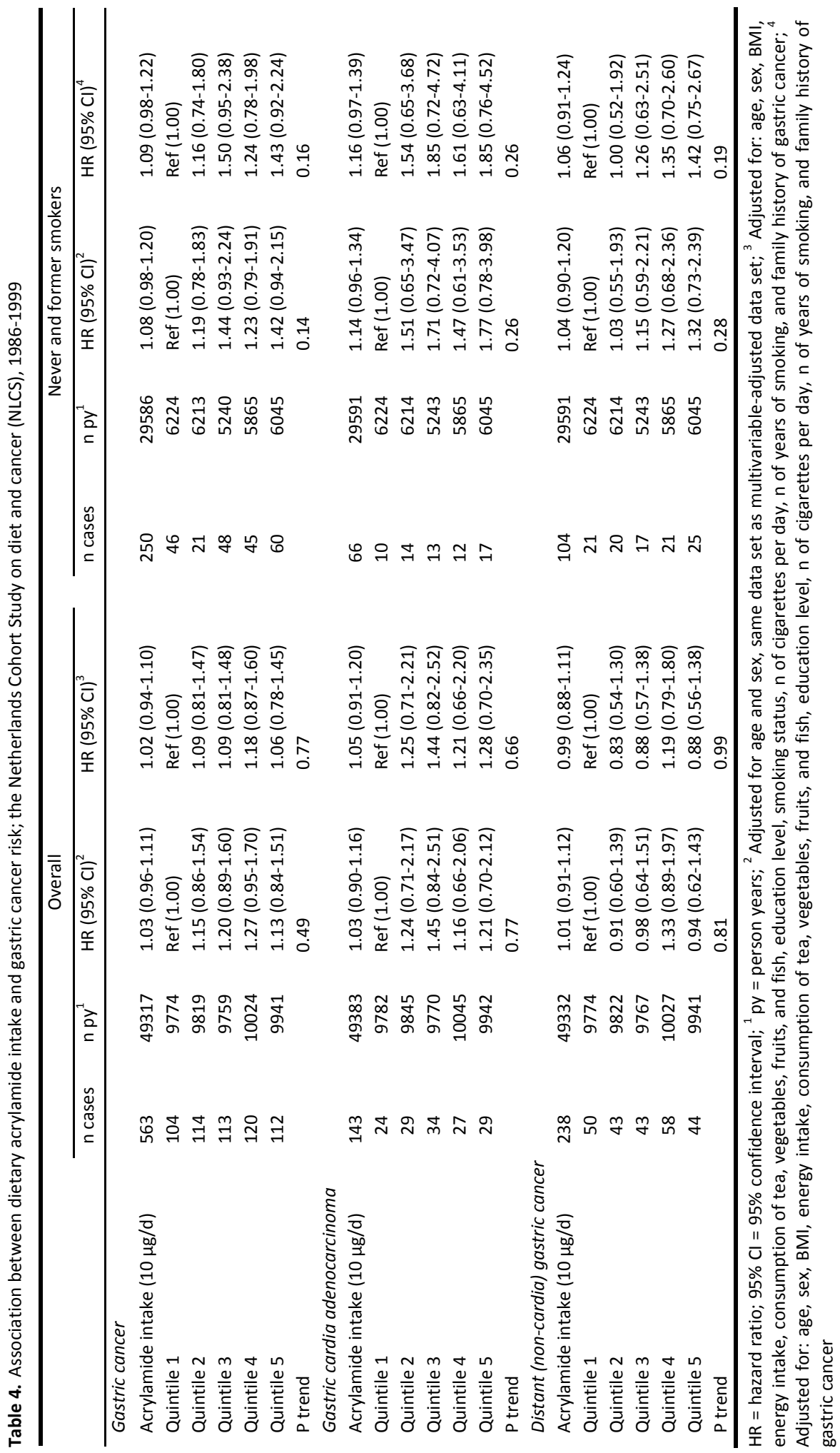




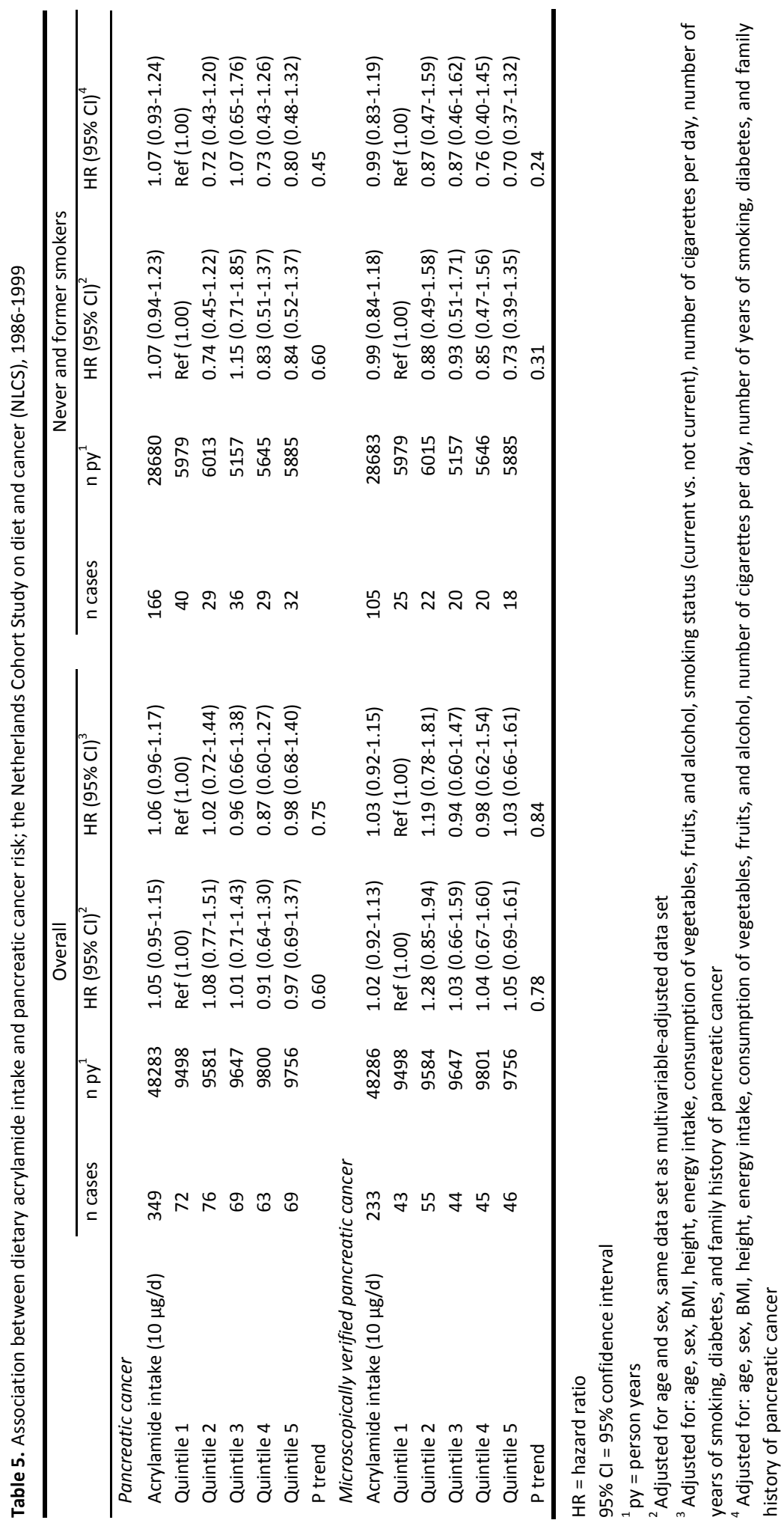




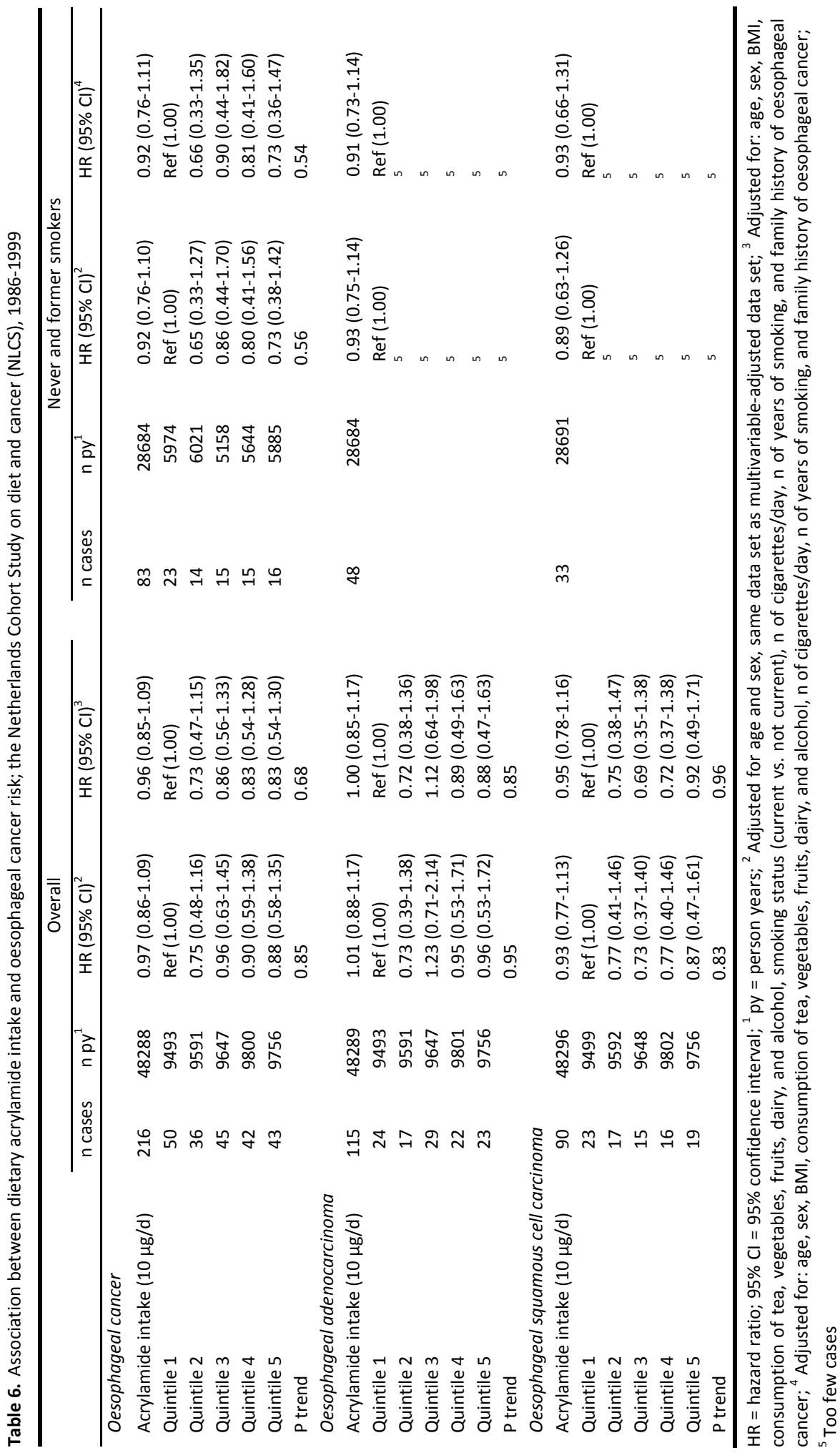




\section{Discussion}

Overall, this prospective cohort study does not give strong support for the hypothesis that dietary acrylamide intake is positively associated with gastrointestinal cancer risk.

The association between dietary acrylamide and colorectal cancer risk was studied in two case-control studies and one prospective cohort study $(16,17,19)$. These studies found no indications of a positive association either. In our study, the HRs across the quintiles for colorectal cancer in never-smokers did not increase linearly (or follow any other clear dose response relationship) and, thus, the significant HRs in some of the quintiles are most likely due to chance.

The association between acrylamide intake and gastric cancer risk has not been studied before. In the present study, no clear indications for a positive overall association were found.

We found no association between dietary acrylamide intake and overall pancreatic cancer risk, contrary to a study on occupational acrylamide exposure $(8,11)$. However, the analyses were poorly adjusted for smoking and other risk factors in that study, which may have biased the results. Furthermore, in an update of this study with longer follow-up and better adjustment for smoking, the acrylamide-associated risk was much reduced and no longer significant (13).

There was no overall association between acrylamide intake and oesophageal cancer risk. This was also observed in the only other study on it, which was a case-control study (16).

The genotoxic action of glycidamide (10) is often mentioned as the predominant mechanism of carcinogenic action in acrylamide cancer risk assessments. The fact that we did not observe an overall association between acrylamide intake and the risk of gastrointestinal tumours, but did observe positive associations between acrylamide intake and endometrial and ovarian cancer risk in a previous study, indicates that disturbance of hormonal balances may also be at the basis of acrylamide carcinogenesis. If acrylamide does indeed exert its carcinogenic effects through a hormonal mechanism, that would explain why no clear overall associations between acrylamide intake and gastrointestinal cancer risk were observed in this study, because sex hormones do not play as clear a role in the etiology of these tumours as they do in the etiology of, e.g., endometrial and ovarian cancer. The fact that a Danish study observed a positive association between acrylamide haemoglobin adducts in the blood and postmenopausal estrogen receptor-positive breast cancer (6) also points toward an effect of acrylamide on hormonal pathways.

The current prospective cohort study has some limitations. FFQs have limitations, as discussed elsewhere (33), but they are the only feasible way to assess dietary intake over a long period in large-scale epidemiological studies. The NLCS FFQ has proved to be both valid (25) and reproducible (26) with regard to nutrients that correlate with acrylamide, such as carbohydrates and fibre.

Within foods, acrylamide concentrations vary greatly, which leads to non-differential misclassification of acrylamide intake, which biases risk estimates toward null. To investigate the extent of misclassification, we estimated the acrylamide content (by using mean 
acrylamide concentrations of individual reported foods) of 39 Dutch 24-hour duplicate diets, which were collected by the Dutch National Institute for Public Health and the Environment in 2004, and correlated this to the analytically measured content and that rendered a Spearman correlation coefficient of 0.82 (Konings et al., see Chapter 2 of this thesis). This indicates that it is feasible to make a sound rank ordering of the acrylamide intake via a 24-hour diet using these mean acrylamide concentrations for foods. The acrylamide values in our food database were derived from foods that were sampled in 2002 and 2005. They may not be completely representative of the foods that were on the market in 1986. This will have resulted in non-differential misclassification of the intake of our cohort, which will then have led to some underestimation of the true associations. We did not query whether the participants bought their foods or prepared them at home. Of the important acrylamide-containing foods, French fries were most likely to be prepared at home in the NLCS population. However, French fries contributed relatively little to the acrylamide intake and to the variance in acrylamide intake in this cohort. Dutch spiced cake, which is an important acrylamide source in this cohort, was not prepared at home. The misclassification that may have arisen from this is probably also non-differential and would also have biased the risk estimates toward null. Despite these potential sources of non-differential misclassification, acrylamide intake was associated with endometrial, ovarian, and renal cell cancer risk in this cohort $(4,5)$. From this, we infer that if in reality acrylamide intake is positively associated with gastrointestinal cancers, the associations will probably be weaker than for endometrial, ovarian, and renal cell cancer.

It has to be borne in mind that the variation in acrylamide intake was largely due to Dutch spiced cake and that coffee was overall the largest dietary source of acrylamide in our study. However, adjustment for Dutch spiced cake and coffee intake in the multivariable-adjusted models did not change the conclusions on the associations between acrylamide intake and colorectal, gastric, pancreatic, and oesophageal cancer risk.

For the effect modification analyses, HRs in many small subgroups were calculated. This makes it likely that some of the observed significant $p$-values for effect modification or significantly increased HRs in subgroups were spurious. Therefore, they should be interpreted cautiously but deserve further investigation in other studies. A high level of non-occupational physical activity was associated with an increased acrylamide-associated risk of colorectal and pancreatic cancer, whereas obese persons had a significantly increased acrylamide-associated risk of pancreatic and oesophageal cancer, although this was based on few obese cases. These factors thus quite consistently modified the risk of some gastrointestinal cancers. This could give support for the hypothesis of glycidamidemediated gastrointestinal carcinogenesis, but physical activity and obesity are also known to influence hormone levels and may thus modify the putative hormonal influences of acrylamide.

This study has some clear strengths, apart from the already mentioned validity and reproducibility of the NLCS FFQ. The acrylamide intake assessment is an important asset of the present study. We used acrylamide concentrations of foods from the Dutch market only and specifically analysed foods that were relevant for the NLCS population. Due to its prospective nature, selection bias is unlikely and differential recall bias is absent. Further- 
more, the association with acrylamide intake was studied for various subgroups of tumours that are known to differ with respect to etiology and risk factors.

In conclusion, overall, we found no indications for a positive association between dietary acrylamide intake and gastrointestinal cancer risk. We encourage other researchers to prospectively investigate the association between dietary acrylamide intake and colorectal, gastric, oesophageal, and pancreatic cancer risk; to perform subgroup analyses for non-smokers; and to study effect modification by factors such as obesity, physical activity, and age. 


\section{References}

1. Tareke E, Rydberg P, Karlsson P, Eriksson S, Tornqvist M. Analysis of acrylamide, a carcinogen formed in heated foodstuffs. J Agric Food Chem. 2002;50:4998-5006.

2. Mottram DS, Wedzicha BL, Dodson AT. Acrylamide is formed in the Maillard reaction. Nature. 2002;419:448-9.

3. Stadler RH, Blank I, Varga N, Robert F, Hau J, Guy PA, Robert MC, Riediker S. Acrylamide from Maillard reaction products. Nature. 2002;419:449-50.

4. Hogervorst JG, Schouten L, Konings EJ, Goldbohm RA, van den Brandt PA. A Prospective Study of Dietary Acrylamide Intake and the Risk of Endometrial, Ovarian, and Breast Cancer. Cancer Epidemiol Biomarkers Prev. 2007;16:2304-13.

5. Hogervorst JG, Schouten $\amalg$, Konings EJ, Goldbohm RA, van den Brandt PA. Dietary acrylamide intake and the risk of renal cell, bladder, and prostate cancer. Am J Clin Nutr. 2008;87:1428-38.

6. Thonning Olesen P, Olsen A, Frandsen H, Frederiksen K, Overvad K, Tjonneland A. Acrylamide exposure and incidence of breast cancer among postmenopausal women in the Danish Diet, Cancer and Health Study. Int J Cancer. 2008;122:2094-100.

7. Sobel W, Bond GG, Parsons TW, Brenner FE. Acrylamide cohort mortality study. Br J Ind Med. 1986;43:7858.

8. Schulz MR, Hertz-Picciotto I, van Wijngaarden E, Hernandez JC, Ball LM. Dose-response relation between acrylamide and pancreatic cancer. Occup Environ Med. 2001;58:609.

9. IARC (1994) Monographs on the evaluation of carcinogen risk to humans: some industrial chemicals, Lyon: International Agency for Research on Cancer.

10. Besaratinia A, Pfeifer GP. A review of mechanisms of acrylamide carcinogenicity. Carcinogenesis. 2007;28:519-28.

11. Marsh GM, Lucas $\amalg$, Youk AO, Schall LC. Mortality patterns among workers exposed to acrylamide: 1994 follow up. Occup Environ Med. 1999;56:181-90.

12. Collins JJ, Swaen GM, Marsh GM, Utidjian HM, Caporossi JC, Lucas LJ. Mortality patterns among workers exposed to acrylamide. J Occup Med. 1989;31:614-7.

13. Marsh GM, Youk AO, Buchanich JM, Kant IJ, Swaen G. Mortality patterns among workers exposed to acrylamide: updated follow up. J Occup Environ Med. 2007;49:82-95.

14. Swaen GM, Haidar S, Burns CJ, Bodner K, Parsons T, Collins JJ, Baase C. Mortality study update of acrylamide workers. Occup Environ Med. 2007;64:396-401.

15. (2002) Health implications of acrylamide in food. Report of a Joint FAO/WHO Consultation. FAO/WHO.

16. Pelucchi C, Galeone C, Levi F, Negri E, Franceschi S, Talamini R, Bosetti C, Giacosa A, La Vecchia C. Dietary acrylamide and human cancer. Int J Cancer. 2006;118:467-71.

17. Mucci LA, Dickman PW, Steineck G, Adami HO, Augustsson K. Dietary acrylamide and cancer of the large bowel, kidney, and bladder: absence of an association in a population-based study in Sweden. Br J Cancer. 2003;88:84-9.

18. Mucci LA, Lindblad P, Steineck G, Adami HO. Dietary acrylamide and risk of renal cell cancer. Int J Cancer. 2004;109:774-6.

19. Mucci LA, Adami HO, Wolk A. Prospective study of dietary acrylamide and risk of colorectal cancer among women. Int J Cancer. 2006;118:169-73.

20. Mucci LA, Sandin S, Balter K, Adami HO, Magnusson C, Weiderpass E. Acrylamide intake and breast cancer risk in Swedish women. Jama. 2005;293:1326-7.

21. Friedman M. Chemistry, biochemistry, and safety of acrylamide. A review. J Agric Food Chem. 2003;51:4504-26.

22. van den Brandt PA, Goldbohm RA, van 't Veer P, Volovics A, Hermus RJ, Sturmans F. A large-scale prospective cohort study on diet and cancer in The Netherlands. J Clin Epidemiol. 1990;43:285-95.

23. Goldbohm RA, van den Brandt PA, Dorant E. Estimation of the coverage of Dutch municipalities by cancer registries and PALGA based on hospital discharge data. Tijdschr Soc Gezondheidsz. 1994;72:80-4.

24. van den Brandt PA, Schouten LJ, Goldbohm RA, Dorant E, Hunen PM. Development of a record linkage protocol for use in the Dutch Cancer Registry for Epidemiological Research. Int J Epidemiol. 1990;19:553-8.

25. Goldbohm RA, van den Brandt PA, Brants HA, van't Veer P, Al M, Sturmans F, Hermus RJ. Validation of a dietary questionnaire used in a large-scale prospective cohort study on diet and cancer. Eur J Clin Nutr. 1994;48:253-65. 
26. Goldbohm RA, van 't Veer P, van den Brandt PA, van 't Hof MA, Brants HA, Sturmans F, Hermus RJ. Reproducibility of a food frequency questionnaire and stability of dietary habits determined from five annually repeated measurements. Eur J Clin Nutr. 1995;49:420-9.

27. Schettgen T, Rossbach B, Kutting B, Letzel S, Drexler H, Angerer J. Determination of haemoglobin adducts of acrylamide and glycidamide in smoking and non-smoking persons of the general population. Int J Hyg Environ Health. 2004;207:531-9.

28. Bergmark E. Hemoglobin adducts of acrylamide and acrylonitrile in laboratory workers, smokers and nonsmokers. Chem Res Toxicol. 1997;10:78-84.

29. Ardies CM, Smith TJ, Kim S, Yang CS. Induction of 4-(methylnitrosamino)-1-(3-pyridyl)-1-butanone (NNK) activation in rat lung microsomes by chronic ethanol consumption and repeated running exercise. Cancer Lett. 1996;103:209-18.

30. Ghanayem BI, Witt KL, Kissling GE, Tice RR, Recio L. Absence of acrylamide-induced genotoxicity in CYP2E1null mice: evidence consistent with a glycidamide-mediated effect. Mutat Res. 2005;578:284-97.

31. Howard LA, Micu AL, Sellers EM, Tyndale RF. Low doses of nicotine and ethanol induce CYP2E1 and chlorzoxazone metabolism in rat liver. J Pharmacol Exp Ther. 2001;299:542-50.

32. Wang Z, Hall SD, Maya JF, Li L, Asghar A, Gorski JC. Diabetes mellitus increases the in vivo activity of cytochrome P450 2E1 in humans. Br J Clin Pharmacol. 2003;55:77-85.

33. Konings EJ, Hogervorst JGF, Schouten LJ, van den Brandt PA (2006) Assessing exposure levels of acrylamide. In: Acrylamide and other hazardous compounds in heat-treated foods (Skog, K. \& Alexander, J., eds.), pp. 214-25. Woodhead Publishing Limited, Cambridge. 


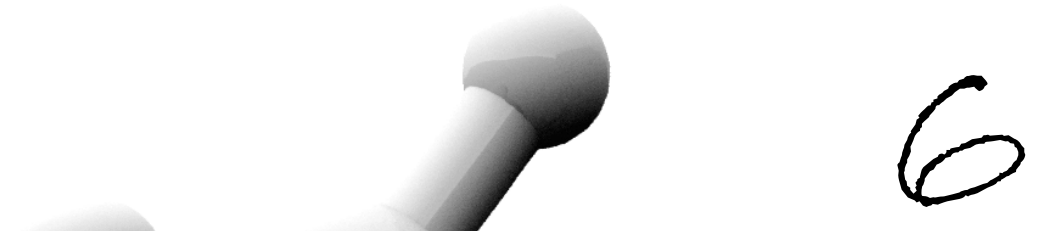

\section{Lung cancer risk in relation to dietary acrylamide intake}

Janneke G.F. Hogervorst

Leo J. Schouten

Erik J.M. Konings

R. Alexandra Goldbohm

Piet A. van den Brandt 


\section{Abstract}

Background Acrylamide is a probable human carcinogen that is present in several heattreated foods. In epidemiological studies, positive associations between dietary acrylamide intake and the risks of endometrial, ovarian, estrogen receptor-positive breast, and renal cell cancers have been observed. The association between dietary acrylamide intake and lung cancer risk is not known.

Methods We conducted a case-cohort study among 58279 men and 62573 women (aged 55-69 years) in the Netherlands Cohort Study on diet and cancer. Intakes of acrylamidecontaining foods and risk factors for cancer were assessed with a self-administered questionnaire at baseline in 1986 and combined with acrylamide concentrations in relevant Dutch foods to assess total dietary acrylamide intake. The number of person-years at risk was estimated by using a random sample of participants from the total cohort that was chosen at baseline $(n=5,000)$. Incident lung cancer cases in the total cohort were detected by computerised record linkages to the Netherlands Cancer Registry and the Netherlands Pathology Registry. Hazard ratios and 95\% confidence intervals (Cls) for the risk of lung cancer associated with acrylamide intakes were estimated using Cox proportional hazards models that controlled for smoking (status, quantity, and duration) and other lung cancer risk factors. All statistical tests were two-sided.

Results After 13.3 years of follow-up (September 17, 1986 up to January 1, 2000), there were 2649 cases of primary, histologically verified lung cancer (International Classification of Diseases for Oncology-3 code: (34) when cases with prevalent cancer at baseline (other than skin cancer) were excluded. The multivariable-adjusted hazard ratio of lung cancer for a $10-\mu \mathrm{g} / \mathrm{d}$ increment of acrylamide intake was $1.03(95 \% \mathrm{Cl}=0.96$ to 1.11$)$ for men and 0.82 (95\% Cl $=0.69$ to 0.96 ) for women. The hazard ratio of lung cancer for the highest (median intake $[\mu \mathrm{g} / \mathrm{d}]$ : men $=37.6$ and women $=36.8$ ) vs the lowest (median intake $[\mu \mathrm{g} / \mathrm{d}]:$ men $=10.8$ and women $=9.5)$ quintile of acrylamide intake was $1.03(95 \% \mathrm{Cl}=0.77$ to $\left.1.39, P_{\text {trend }}=.85\right)$ for men, and $0.45\left(95 \% \mathrm{Cl}=0.27\right.$ to $\left.0.76, P_{\text {trend }}=.01\right)$ for women. The inverse association in women was strongest for adenocarcinoma (hazard ratio for highest vs lowest tertile of intake $=0.40,95 \% \mathrm{Cl}=0.21$ to $0.78 ; P_{\text {trend }}=.01$ ).

Conclusions Acrylamide intake was not associated with lung cancer risk in men but was inversely associated in women, most strongly for adenocarcinoma. This finding suggests that acrylamide is involved in human carcinogenesis through pathways other than genotoxicity. 


\section{Introduction}

Acrylamide was found several years ago to be present in commonly consumed carbohydrate-rich heated foods (1) such as French fries and potato crisps and is classified as a probable human carcinogen based on results from animal studies (2). Recently, an advisory group of the International Agency for Research on Cancer (IARC) gave high priority to assessment of acrylamide as a human carcinogen in future IARC Monograph series (3), probably because of the outcomes of some recent epidemiological studies on acrylamide and cancer risk (4-6).

Animal studies have shown positive dose-response relationships between acrylamide exposure and cancer in multiple organs and tissues (7-10), including oral tissues, thyroid gland, and mammary gland, in the rat, and lung and skin in the mouse. Recently, two prospective epidemiological studies have shown positive associations between acrylamide exposure and cancer risk. In one of those studies, we observed that increased dietary acrylamide exposure was positively associated with the risk of postmenopausal endometrial and ovarian cancers (but not with the risk of postmenopausal breast cancer) (4), as well as with the risk of renal cell cancer (but not with the risk of bladder and prostate cancer) (5). In the other study, a Danish group observed a positive association between acrylamide exposure and postmenopausal estrogen receptor-positive breast cancer risk using acrylamide-haemoglobin adducts for the assessment of acrylamide exposure (6). More prospective epidemiological studies on the association between acrylamide intake and cancer risk are needed, especially for cancer sites that have not yet been studied, such as the lung.

Here we report the first prospective epidemiological study to examine the association between dietary acrylamide intake and lung cancer risk.

\section{Methods}

\section{Study design and participants}

This study was conducted within the prospective Netherlands Cohort Study (NLCS) on diet and cancer, which started in September 1986 with the inclusion of 58279 men and 62573 women aged 55-69 years who were randomly sampled from Dutch municipal registries (11). At baseline, study participants were sent a questionnaire on diet and other risk factors for cancer, such as smoking and physical activity, that was designed to be selfadministered. Participants were informed that by returning the completed questionnaire they would be giving their consent to participate in a study of the etiology of cancer in relation to diet. This procedure of informed consent was approved by the Medical Ethics Committees of the University Hospital Maastricht and the Netherlands Organisation for Applied Scientific Research.

A case-cohort approach (12) was used for data processing and analysis; cases were derived from the entire cohort, and the number of person-years at risk for the entire cohort was estimated from a subcohort of 5000 men and women who were randomly 
sampled from the full cohort at baseline. Incident cases of lung cancer in the total cohort were detected by computerised record linkages to the Netherlands Cancer Registry and the Netherlands Pathology Registry. The completeness of cancer follow-up through linkage with these cancer registries is at least $96 \%$ (13), and follow-up of the subcohort at the end of the 13.3-year follow-up period (September 17, 1986, up to January 1, 2000) was nearly $100 \%$ complete (only two male subcohort members were lost to follow-up). Further details on the design of the study and methods of follow-up have been published previously $(11,14,15)$.

Case subjects and subcohort members were excluded from this analysis if they had been diagnosed with any other cancer (except skin cancer) at baseline and if their dietary data were incomplete or inconsistent. Figure 1 shows the selection and exclusion steps that resulted in the numbers of case subjects and subcohort members that were included in the analysis.

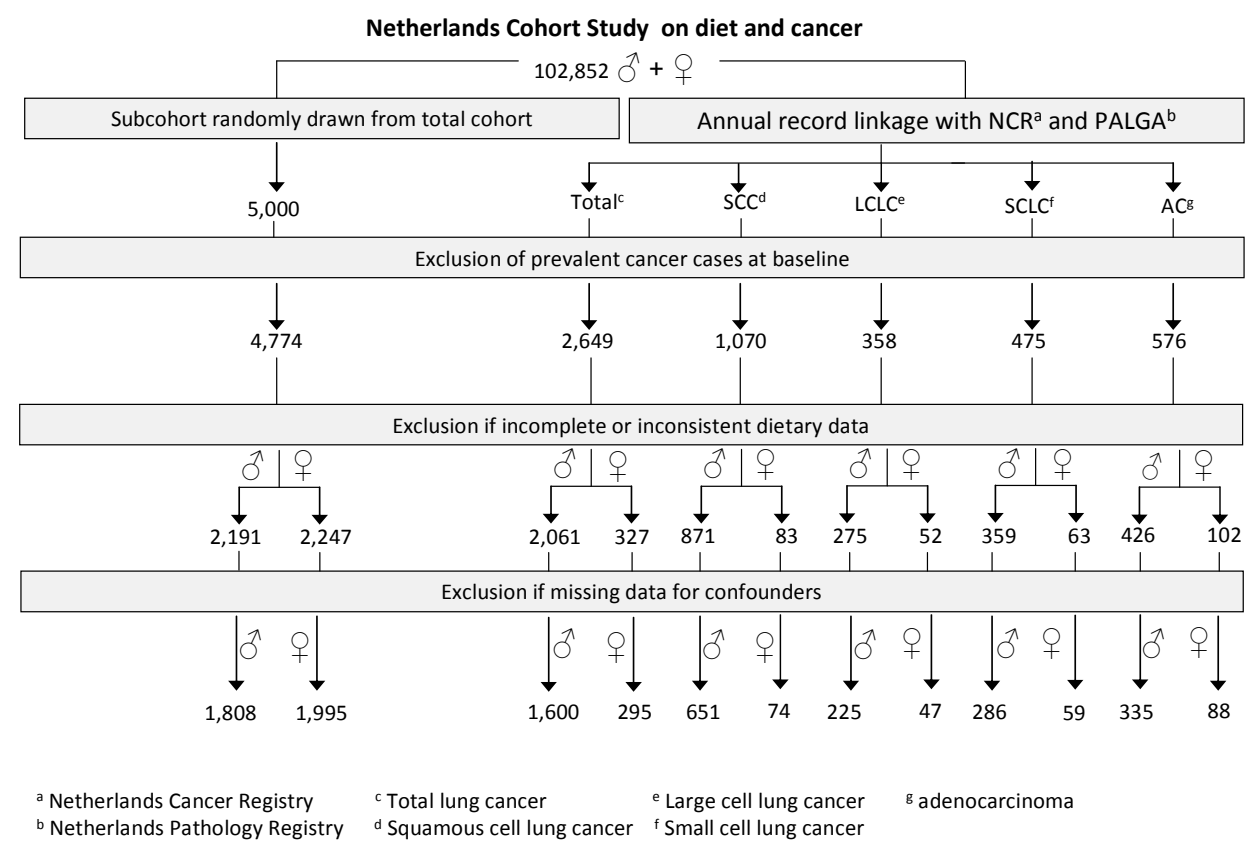

Figure 1. Flow diagram of subcohort members and cases on whom the analyses were based

According to the formula of Cai and Zeng (16), the power was $80 \%$ to detect a statistically significant ( $\alpha=.05$ ) association with a hazard ratio (HR) of 1.35 or higher (analysing all male case subjects) or 1.90 or higher (for the smallest case group in men, those with large cell tumours), when comparing the highest with the lowest quintile of acrylamide intake. For women the power was $80 \%$ to detect a statistically significant ( $\alpha=.05$ ) association with an HR of 1.65 or higher for overall lung cancer or 3.2 or higher for large cell 
tumours (the smallest case group in women), when comparing the highest with the lowest quintile of acrylamide intake.

\section{Acrylamide intake assessment}

The NLCS food-frequency questionnaire contained questions on 150 food items $(14,15)$. It queried the habitual intake, in terms of frequency, and for most foods, portion size, of foods consumed by the participant during the year preceding baseline. The major dietary sources of acrylamide in the Netherlands in 1998 (determined through a National Food Consumption Survey) were estimated to be potato crisps ( $31 \%$ of the total), French fries (21\%), Dutch spiced cake (16\%), coffee (13\%), bread (10\%), and cookies (4\%) (17). These foods were all included as separate items in the NLCS FFQ, and thus the most important dietary acrylamide sources in the Netherlands were taken into account in the acrylamide exposure assessment.

We used data from the Dutch Food and Consumer Product Safety Authority on acrylamide concentrations in foods that were sampled from Dutch shops in 2002 and 2005 to obtain an intake estimate representative for our study population. Chemical acrylamide measurements in cookies were done in several types of cookies that are known to be eaten most frequently by a population comparable to the NLCS, according to information from the development phase of the questionnaire. The acrylamide level that was used for cookies in this acrylamide intake estimation was based on the acrylamide level of the specific types of cookies weighted by the relative frequency of consumption of the NLCScomparable population. The same was done to estimate acrylamide concentrations for other composite food items, such as pies and chocolates.

We did a validation study to investigate whether using mean acrylamide concentrations for foods that vary widely in their acrylamide content results in a sound estimate of total acrylamide intake (EJM Konings, JGF Hogervorst, L van Rooij, LJ Schouten, EA Sizoo HP van Egmond, see Chapter 2 of this thesis). For this validation study, we used 39 collected 24-hour duplicate diets that were collected by the Dutch National Institute for Public Health and the Environment in 2004. A 24-hour duplicate diet is the collection of duplicate portions of everything eaten and drunk during 24 hours by an individual. The 39 participants in the duplicate diet study were asked to keep a diary in which they reported the amounts of specific foods that they ate during the 24 hours; we used the information in those diaries to estimate the acrylamide content of the 24-hour diets as the amount of specific food multiplied by the mean acrylamide level of a specific food that was also used for the NLCS acrylamide intake assessment. Subsequently, the acrylamide concentrations of the duplicate diets were chemically analysed by liquid chromatography-tandem mass spectrometry using the method of Rosén and Hellenas (18) and correlated to the acrylamide content estimated from the diaries, which rendered a Spearman correlation coefficient of $.82(P<.001)$. This good correlation indicates that it is feasible to make a reliable rank ordering of acrylamide intake via a 24-hour diet by using mean acrylamide concentrations for individual foods. Further details of the intake assessment, including concentrations of acrylamide in foods, are presented elsewhere (4). 


\section{Statistical analysis}

Confounders were selected in two steps: some were chosen a priori to be included in the models. Others were included only if they changed the age- and sex-adjusted hazard ratio of lung cancer for a $27-\mu \mathrm{g} / \mathrm{d}$ increment of acrylamide intake $(27 \mu \mathrm{g} / \mathrm{d}$ is the difference between the $10^{\text {th }}$ and $90^{\text {th }}$ percentile of acrylamide intake of the subcohort) by more than $10 \%$ compared with the age- and sex-adjusted hazard ratio. The a priori-selected covariables were: age (years); sex; family history of lung cancer (yes or no); smoking status (current or not current); number of cigarettes smoked per day; duration of smoking (years); body mass index (BMI, $\left.\mathrm{kg} / \mathrm{m}^{2}\right)$; energy intake $(\mathrm{kcal} / \mathrm{d})$; consumption of vegetables $(\mathrm{g} / \mathrm{d})$, fruits $(\mathrm{g} / \mathrm{d})$ and alcohol $(\mathrm{g} / \mathrm{d})$; educational level (primary school, lower vocational school, intermediate vocational/high school, or higher vocational school/university); and, for the analyses of men, pipe and cigar smoking (never or former or current, number smoked per day, and number of years of smoking for pipe and cigar separately). The following variables were checked for confounding with the $10 \%$ change criterion that was described above: height $(\mathrm{cm})$, nonoccupational physical activity $(\mathrm{min} / \mathrm{d})$, consumption of dairy $(g / d)$, meat $(g / d)$, processed meat $(g / d)$, and fish $(g / d)$, intake of vitamins $A(m g / d)$ and $C(m g / d)$, $\alpha$-carotene $(\mu \mathrm{g} / \mathrm{d}), \beta$-carotene $(\mu \mathrm{g} / \mathrm{d})$, selenium $(\mu \mathrm{g} / \mathrm{d})$, folate $(\mu \mathrm{g} / \mathrm{d})$, niacin $(\mathrm{mg} / \mathrm{d})$, total fat $(\mathrm{g} / \mathrm{d})$ saturated fat $(\mathrm{g} / \mathrm{d})$, trans-fatty acid $(\mathrm{g} / \mathrm{d})$, carbohydrates $(\mathrm{g} / \mathrm{d})$, and fibre $(\mathrm{g} / \mathrm{d})$; and use of vitamin A supplements (yes or no). In separate analyses, we additionally adjusted for the following passive smoking variables: smoking by the parents in the period that the participant lived with his or her parents (no, both, mother only, father only); smoking by the partner in the presence of the participant (never or former or current), and number of hours per day spent in a smoky room. In other separate analyses, we additionally adjusted for consumption of the five most important dietary sources of acrylamide in this cohort: coffee $(g / d)$, Dutch spiced cake $(g / d)$, cookies $(g / d)$, potato crisps (g/d), and French fries (g/d) (4).

Compared with nonsmokers, smokers have, on average, three to four times higher levels of acrylamide-haemoglobin adducts, which are a biomarker for exposure to acrylamide (19). For this reason, and because smoking is such an important risk factor for lung cancer, subgroup analyses were done for never-smokers. For men, this subgroup included cigar and pipe smokers, and in those analyses, we adjusted for pipe and cigar smoking. There were virtually no female cigar or pipe smokers in this study population (five cigar smokers in the subcohort, and one cigar smoker and one pipe smoker among the large-cell lung cancer case subjects), and therefore we did not adjust the hazard ratios for women for pipe and cigar smoking. Hazard ratios and $95 \%$ confidence intervals (Cls) were obtained through Cox proportional hazards regression (20) using STATA software (release 9.2, 2005, STATA Corporation, College Station, TX) with person-years at risk as the time metric. Scaled Schoenfeld residuals were used to test the proportional hazards assumption (21). The assumption was not violated.

Additional variance introduced by sampling a subcohort from the cohort was taken into account by estimation of standard errors using the robust Huber-White sandwich estimator, a method similar to the variance-covariance estimator of Barlow (12). Tests for dose-response trends were done by fitting the median acrylamide intake per quintile or 
tertile as a continuous variable. We considered 100 and 60 case subjects as the minimum number needed to do analyses with acrylamide exposure categorised into quintiles and tertiles, respectively, and assumed that 20 case subjects were needed to analyse acrylamide exposure as a continuous variable.

To examine the influence of preclinical disease on the results, the analyses were also done with exclusion of case subjects with cancer detected in the first 2 years of follow-up. We also did subgroup analyses for participants who had no occupational exposure to biological dust, mineral dust, or gases or fumes [as defined according to the communitybased job exposure matrix (ALOHA JEM) that was developed to translate occupation into none, low or high exposure to biological dust, mineral dust, or gases or fumes (22)]. Effect modification of the association between acrylamide intake and lung cancer by other variables was tested using Wald $\chi^{2}$ tests. The variables that were tested for effect modification were selected according to their ability to modify the activity of CYP2E1 (23-26), the enzyme that converts acrylamide to genotoxic glycidamide (27). These variables were age (55-59, 60-64, 65-69 yrs), diabetes (ever or never diagnosis of diabetes), obesity (BMI $>30 \mathrm{~kg} / \mathrm{m}^{2}$ ), smoking (smoking status and both duration of smoking in years and number of cigarettes smoked per day), alcohol consumption $(0,>0$ to $5,>5 \mathrm{~g} / \mathrm{d}$ ), and physical activity $(<30,30$ to $<60,60$ to $<90, \geq 90 \mathrm{~m} / \mathrm{d})(24-26,28)$. All reported $P$ values were from two-sided tests. A $P$ value less than .05 was considered statistically significant.

\section{Results}

After 13.3 years of follow-up (September 17, 1986 up to January 1, 2000) there were 2388 cases of primary, histologically verified lung cancer (International Classification of Diseases for Oncology-3 code: C34) when cases with prevalent cancer (other than skin cancer) at baseline and cases with incomplete or inconsistent dietary data were excluded. The characteristics of the subcohort and the lung cancer cases by sex and by histological subtype are shown in Table 1. Apart from the expected higher frequency, quantity, and duration of smoking among case subjects than among subcohort members, there were clear differences in fruit and alcohol intake. Case subjects, regardless of the type of lung cancer that they had, consumed less fruit and had a higher alcohol intake than subcohort members. In addition, among the male participants, case subjects, regardless of lung cancer type, were older than subcohort members were, whereas among the female participants this was not the case.

The interaction between acrylamide intake and sex was statistically significant or borderline statistically significant in most case subject groups $\left(P_{\text {interaction }}=.06, .08\right.$, and $<.01$ for all case subjects with any lung cancer, small cell carcinoma, and adenocarcinoma, respectively). Therefore, the hazard ratios for lung cancer according to histological subtype are shown separately for men (Table 2 ) and women (Table 3).

In men (smokers and non-smokers combined), acrylamide intake was not associated with the risk of lung cancer overall. The multivariable-adjusted hazard ratio of lung cancer for a $10-\mu \mathrm{g} / \mathrm{d}$ increment of acrylamide intake was $1.03(95 \% \mathrm{Cl}=0.96$ to 1.11$)$ and there was no 


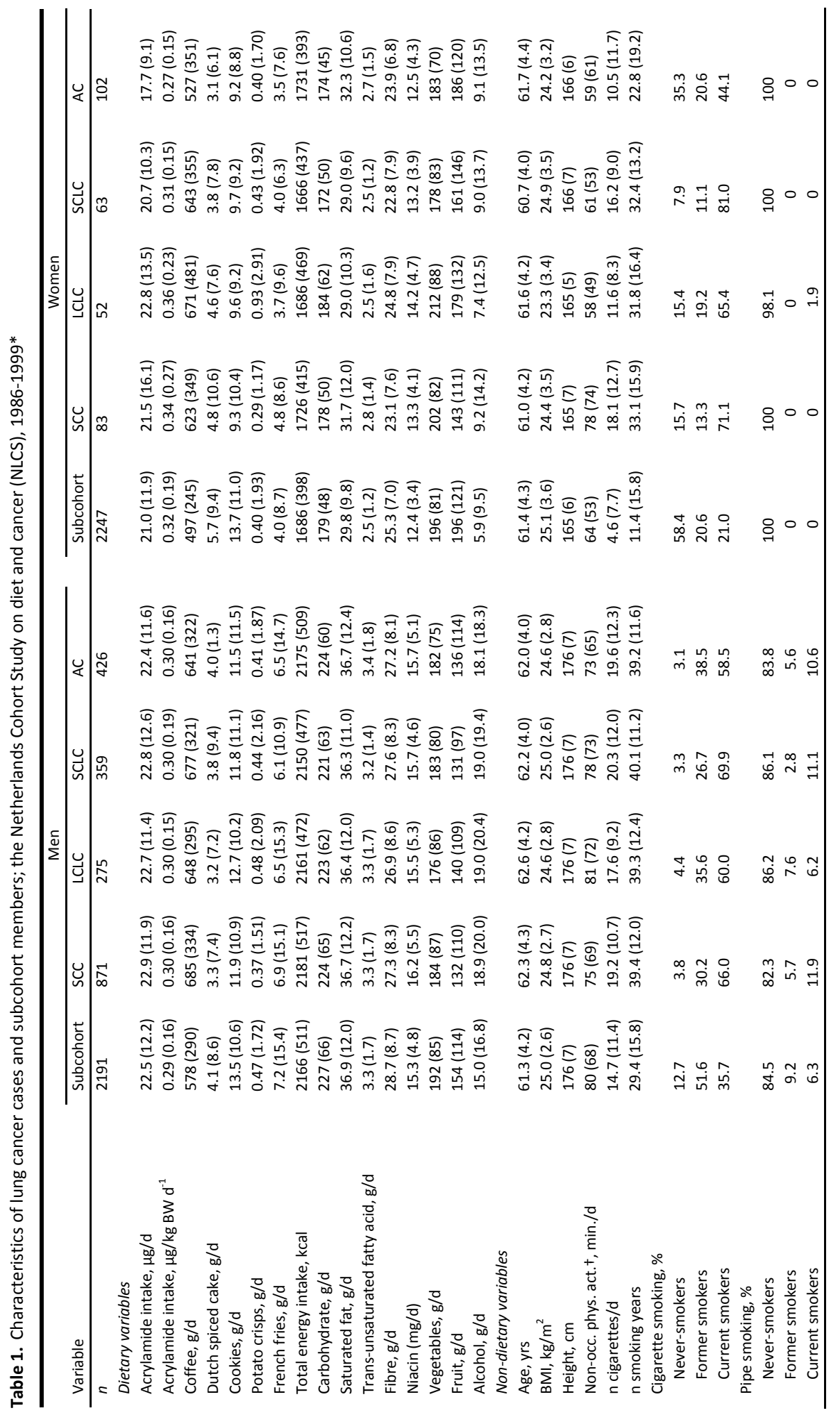


Dietary acrylamide intake and lung cancer risk

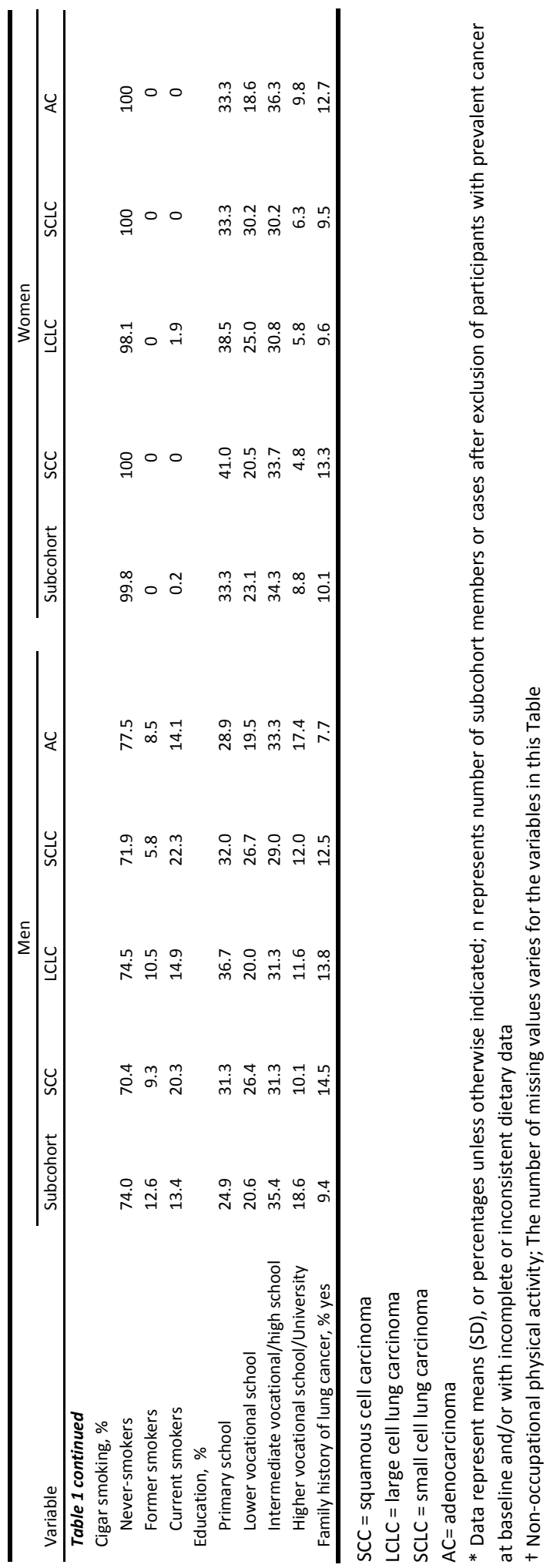




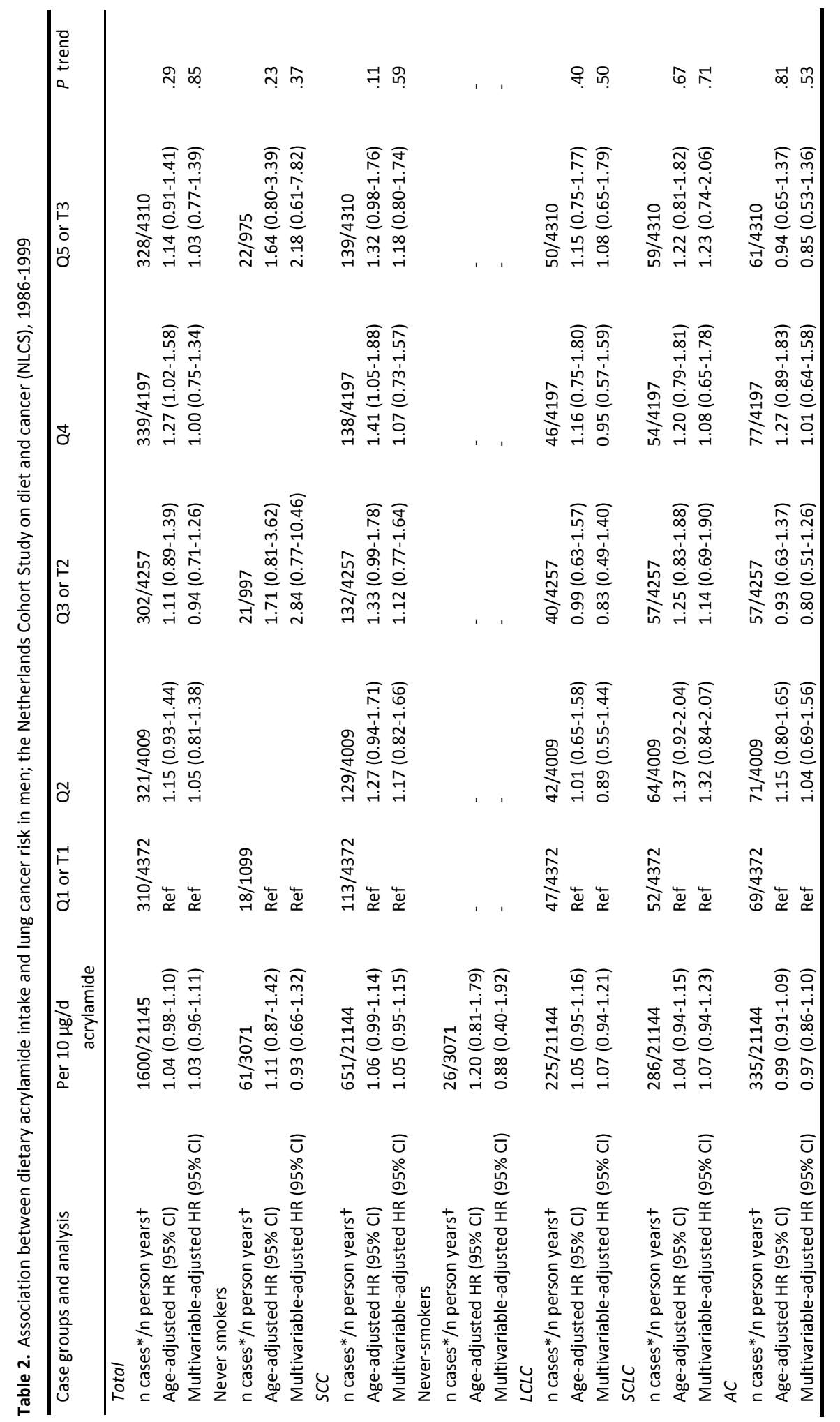




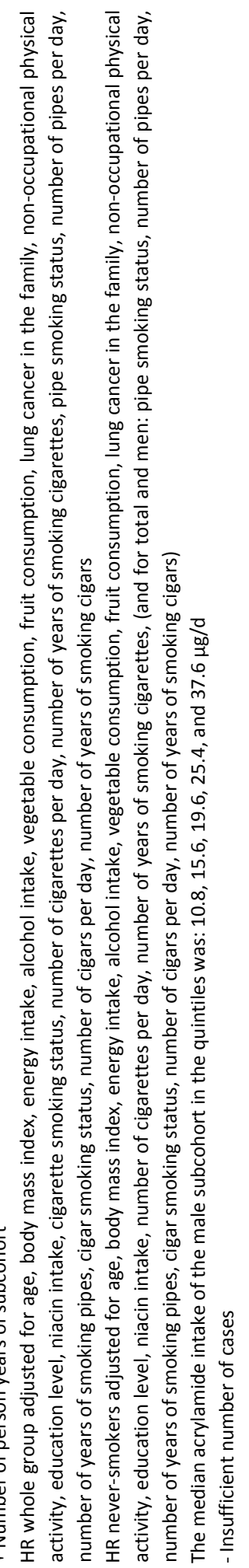




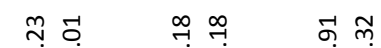

กิ ธี

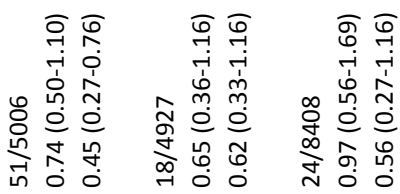

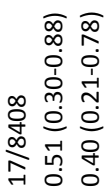

నิ ณू

$\infty$ 它

$\infty$

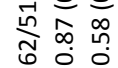

ฮั

苛

ธ

总

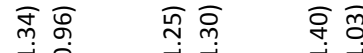

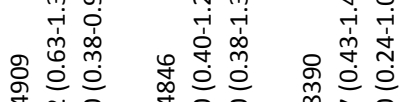

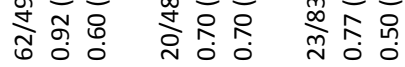

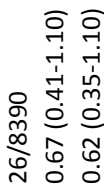

Щิ

$\rightarrow-i$

กิ่

นิ응

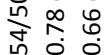

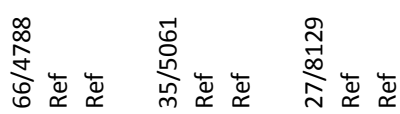

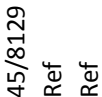



\section{(18}

กิ

오 구

윽

क人

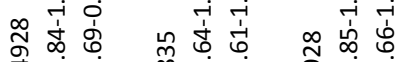

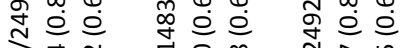

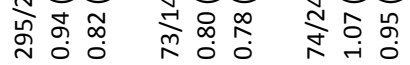

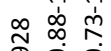
赵 $\infty$ 它字

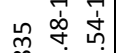
gे

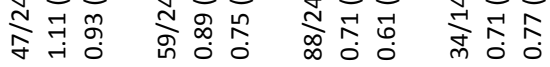




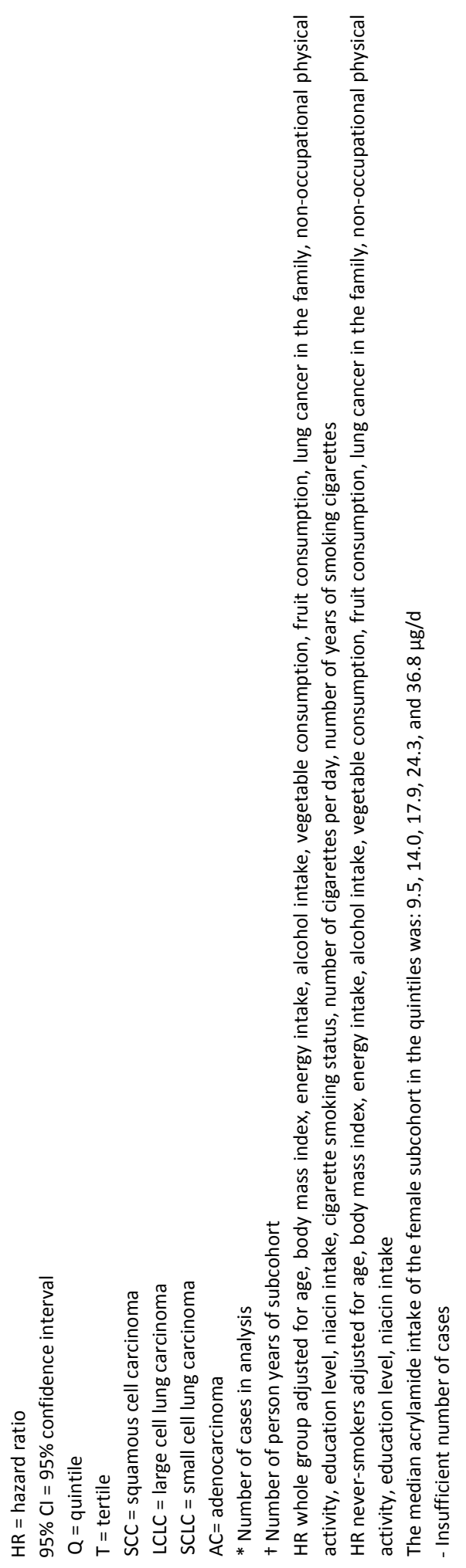




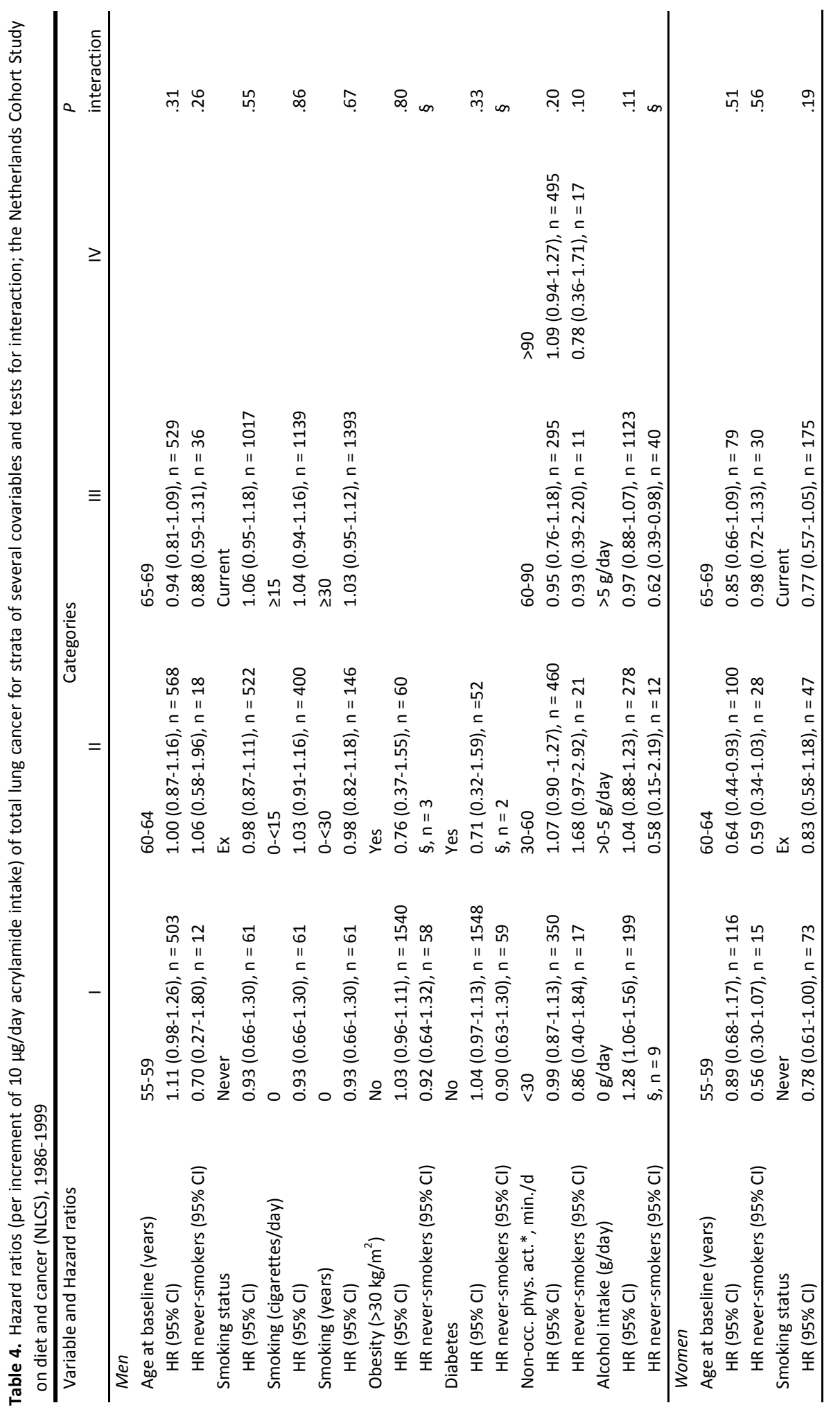




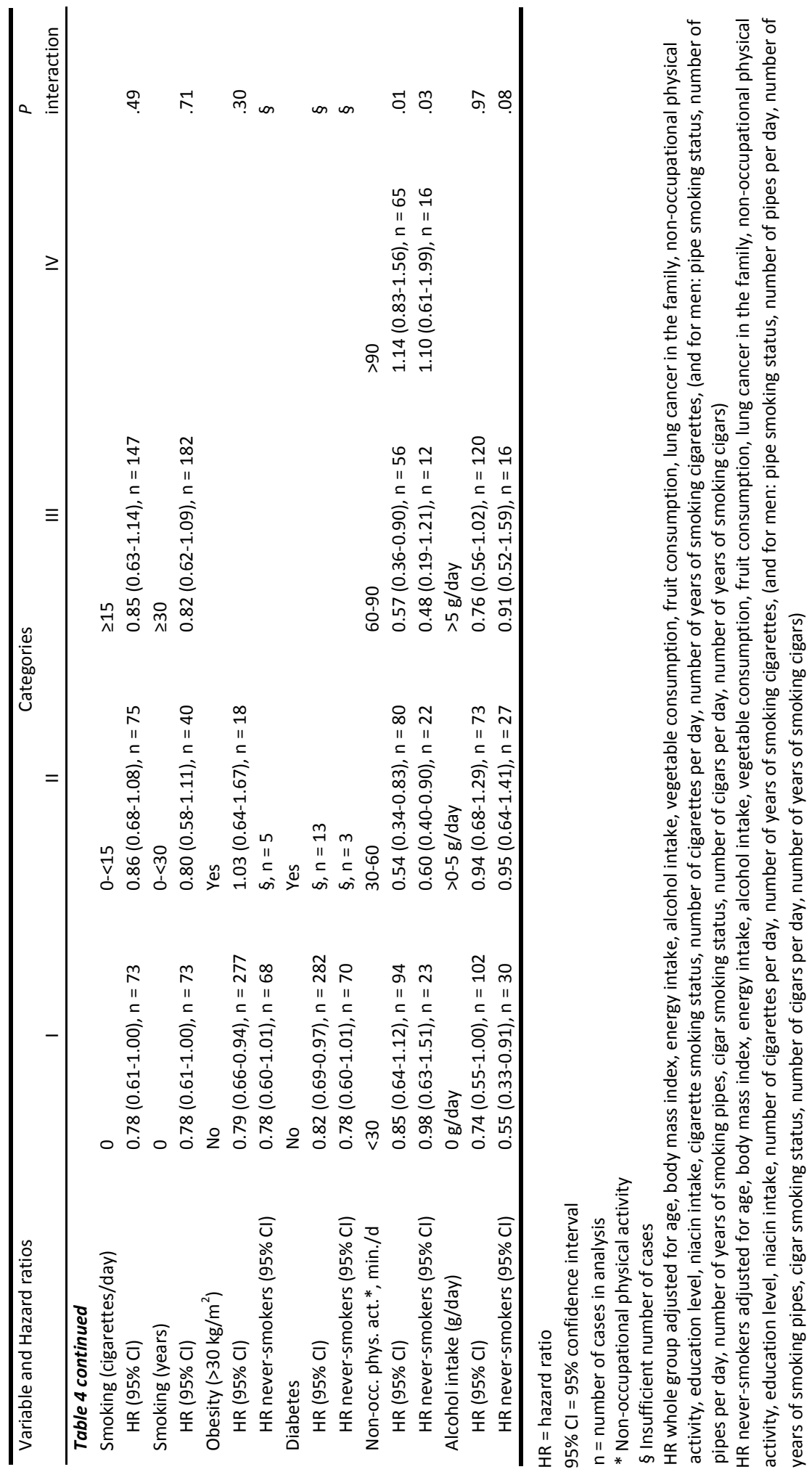


trend over the quintiles of acrylamide intake. Acrylamide was also not associated with the risk of any of the histological subtypes of lung cancer. In male never-smokers, there were increased hazard ratios in the second and third tertiles of acrylamide intake, but the $95 \%$ confidence intervals were wide. When acrylamide intake was treated as a continuous variable, there was no evidence of an increased risk of lung cancer $(\mathrm{HR}=0.93 ; 95 \% \mathrm{Cl}=$ 0.66 to 1.32). Because of the small number of male never-smokers, it was not possible to investigate if the increased risks in this group were due to a particular subtype of lung cancer.

In women, there was a statistically significantly decreased risk of overall lung cancer associated with increasing acrylamide intake, both for the continuous acrylamide variable (multivariable-adjusted HR per $10 \mu \mathrm{g} / \mathrm{d}$ increment of acrylamide intake $=0.82 ; 95 \% \mathrm{Cl}=$ 0.69 to 0.96 ) and across the intake quintiles (multivariable-adjusted HR for highest vs lowest quintile $=0.45 ; 95 \% \mathrm{Cl}=0.27$ to $0.76 ; P_{\text {trend }}=.01$ ). For never-smoking women, there was also an inverse association between acrylamide intake and overall lung cancer risk, with a borderline statistically significant multivariable-adjusted hazard ratio for the continuous acrylamide variable ( $\mathrm{HR}=0.78 ; 95 \% \mathrm{Cl}=0.61$ to 1.00$)$ and an inverse trend across the tertiles of acrylamide intake with a statistically nonsignificant $P$ for trend. Within the female subgroups based on histological subtypes, a statistically nonsignificant inverse trend across the tertiles of acrylamide intake was observed for squamous cell cancer (although the hazard ratio for the continuous acrylamide variable was not significantly decreased) and most strongly for adenocarcinoma, for which the hazard ratio in the third tertile compared with the first tertile was $0.40\left(95 \% \mathrm{Cl}=0.21\right.$ to $\left.0.78 ; P_{\text {trend }}=.01\right)$.

The null associations in men and the inverse associations in women remained unchanged when we additionally adjusted for passive smoking by the parents and by the domestic partner and for the number of hours per day spent in a smoky room (data not shown). In addition, adjustment for intake of coffee, Dutch spiced cake, cookies, potato crisps, or French fries did not result in substantially different hazard ratios (data not shown).

The results in both men and women did not change substantially when we excluded case subjects who were diagnosed during the first 2 years of follow-up (data not shown). In addition, in subgroup analyses among participants who were never occupationally exposed to biological dust, mineral dust, or gases or fumes, the results were not substantially changed (data not shown).

In men, there were no statistically significant $P$ values for interaction for any of the variables tested for total lung cancer (Table 4). However, men with the lowest alcohol intake had a statistically significantly increased acrylamide-associated risk of lung cancer when acrylamide intake was treated as a continuous variable (HR per $10-\mu \mathrm{g} / \mathrm{d}$ increment $=$ $1.28 ; 95 \% \mathrm{Cl}=1.06$ to 1.56$)$.

In women, the $P$ value for interaction between non-occupational physical activity and acrylamide was statistically significant both in the total group of women and in the neversmoking women, but the acrylamide-associated risk of lung cancer did not increase or decrease linearly over the categories of non-occupational physical activity but instead followed a U-shaped curve. Furthermore, among never-smoking women, those in the 
lowest category of alcohol intake had a lower acrylamide-associated risk of lung cancer risk than those in the higher categories of alcohol intake, with a borderline statistically significant $P$ for interaction $(P=.08)$.

\section{Discussion}

This prospective cohort study did not provide evidence of a positive association between dietary acrylamide intake and lung cancer risk in men.

In women, we observed a statistically significant inverse association between acrylamide intake and lung cancer risk; this association was strongest for adenocarcinoma. The inverse association in women was robust in that it remained unchanged after exclusion of cases diagnosed during the first 2 years of follow-up and after adjustment for exposure to passive smoke or for consumption of foods that are important sources of dietary acrylamide in this cohort. Furthermore, after multivariable-adjustment, the hazard ratios were more decreased than the age-adjusted hazard ratios, which implies that residual confounding by any of the included covariables (eg, smoking) is unlikely. In never-smoking women, the hazard ratios were decreased as well.

Our results are not in line with two mouse studies. In two strains of mice (male and female $A / J$ mice and female Swiss-ICR mice), an increased incidence of lung cancer was observed after administration of acrylamide by gavage or intraperitoneal injection $(8,9)$. To our knowledge, this is the first time that dietary acrylamide intake has been studied in relation to human lung cancer risk. Epidemiological studies on occupational acrylamide exposure have observed an increased risk of respiratory tract cancer (28-30), but this finding was ascribed to concurrent exposure to organic dyes. In addition, there was no evidence of a dose-response relationship, a negative finding that does not support a causal association between acrylamide exposure and respiratory tract cancer risk. Furthermore, these epidemiological studies lacked appropriate control for confounders, such as smoking, and the study populations included virtually only men.

Our study has some limitations that should be described. First, an important component of any epidemiological study on the association between dietary acrylamide intake and cancer risk is the validity and reliability of the exposure assessment. A number of sources of non-differential misclassification of the acrylamide intake may have occurred in this study. For example, we measured acrylamide sampled in Dutch shops in 2002 and 2005 , but the study participants consumed foods that were on the market before those dates. We did not ask participants whether the foods they consumed were purchased preprepared or were prepared by them at home. Of the important acrylamide-containing foods, French fries were most likely to be prepared at home in the NLCS population. However, French fries contributed relatively little to the acrylamide intake and to the variance in acrylamide intake in this cohort (4). Furthermore, acrylamide concentrations vary considerably within food items, such as French fries. However, all of these sources of nondifferential misclassification would bias the true association toward the null, so that the inverse association for women that we observed might be even stronger. On the other 
hand, our acrylamide intake assessment also has an important strength in that we only used acrylamide concentrations in foods sampled from Dutch shops and specifically sampled and analysed foods that were relevant for the NLCS population.

Second, although we checked extensively for variables that confounded the association between acrylamide intake and lung cancer risk, confounding by unknown confounders might be an alternative explanation for the observed associations.

Finally, for the interaction analyses, the fact that we calculated hazard ratios for many small subgroups makes it likely that some of the observed statistically significant $P$ values for interaction or hazard ratios in subgroups did not represent true associations, and should therefore be interpreted with caution. The observations reported here need confirmation or refutation from other epidemiological studies in independent cohorts.

It is assumed that genotoxic compounds have no threshold below which exposure to this compound does not entail a risk of cancer. There are several indications that acrylamide is genotoxic in vitro and in vivo, mainly after it is metabolised to glycidamide. Acrylamide causes clastogenic effects (chromosome aberrations, micronuclei, sister chromatid exchanges); glycidamide induces the same effects at lower doses and forms DNA adducts, which can lead to point mutations (31). Because of this presumed genotoxicity, linear extrapolation from high chronic doses in rats to low chronic doses in humans is applied in acrylamide risk assessment. The outcome of these extrapolations is that an acrylamide intake of $1 \mu \mathrm{g} / \mathrm{kg}$ body weight per day in humans corresponds to a relative risk of cancer in humans of between 1.006 and 1.05 (32) (in the Netherlands, the mean intake is approximately $0.5 \mu \mathrm{g} / \mathrm{kg}$ body weight per day) (17). The increased relative risks of endometrial, ovarian, renal cell, and estrogen receptor-positive breast cancer observed in recent epidemiological studies of acrylamide exposure (4-6) are at least 10- to 100-fold higher than what would have been expected from the animal studies. Although these epidemiological results need to be confirmed, they raise the possibility that risk extrapolation based on animal results might underestimate human cancer risk associated with acrylamide exposure instead of rendering conservative risk estimates, as is often presumed to be the case when using linear dose extrapolation. This underestimation might be due to a steeper dose-response relationship at lower doses than at higher doses or to entirely different mechanisms that operate at different dose levels or in different species. Epidemiological studies are important, because they investigate the risks in the relevant species at the relevant doses.

The inverse association between dietary acrylamide intake and the risk of lung cancer that we observed for women may indicate that acrylamide is involved in human carcinogenesis through another pathway besides genotoxicity. The theory of a hormonal pathway of acrylamide carcinogenicity was first suggested because of the predominantly hormonesensitive sites at which cancer occurred after administration of acrylamide in animal studies $(7,10)$. Furthermore, the frequency distribution of glycidamide DNA adducts across different tissues did not match the frequency with which cancers arose in particular tissues in animals (33), suggesting that genotoxicity may not be the only relevant cancercausing characteristic of acrylamide. Consistent with the possibility that hormonal factors might be at the basis of the carcinogenic action of acrylamide, we observed positive 
associations between acrylamide intake and endometrial and ovarian cancer risk (4), and a Danish study observed a positive association between acrylamide haemoglobin adducts in the blood and the risk of estrogen receptor-positive breast cancer (6). The established risk factors for these cancers are predominantly related to hormonal levels.

Studies in rats have produced conflicting evidence for the role of acrylamide in the disruption of endocrine systems. Acrylamide was shown to influence levels of prolactin, testosterone, estrogen, and progesterone (34-36), but in a study of male rats, no clear influence of acrylamide on the hypothalamus-pituitary-thyroid axis was observed (37). In the latter study however, no sex steroid hormones were studied. A recent in vitro study on glycidamide-induced gene expression in human breast and colon cancer cells showed increased expression of genes that catalyse the conversion of inactive androgen and estrogen precursors to active forms, such as testosterone, and $17 \beta$-estradiol (38).

Several lines of evidence indicate the involvement of sex hormones in lung cancer etiology. Normal human lung tissue and lung tumour tissue contain estrogen [especially estrogen receptor- $\beta$ (ER- $\beta$ ) in contrast to for instance endometrial tissues, which contain mainly ER- $\alpha$ (40)], progesterone, and androgen receptors, and the expression of the receptors differs between men and women and among lung cancer subtypes (40-42). Adenocarcinomas, for example, express ER- $\beta$ to a higher extent than squamous cell carcinomas (42). Several epidemiological studies have looked at the association between postmenopausal hormone treatment and female lung cancer risk, and some of these studies showed a decreased risk of postmenopausal hormone treatment $(43,44)$. It is interesting that within the NLCS the associations between postmenopausal hormone treatment, oral contraceptive use, and BMI and lung cancer risk were opposite to those with endometrial and ovarian cancer, for which we observed positive associations with acrylamide exposure (4). On the basis of the above-mentioned, albeit still fragmented observations (sex hormone level changes in rats, increased expression of sex hormone precursor genes, estrogen receptors in lung tissue, and associations between reproductive factors and lung cancer risk), we hypothesise that acrylamide may alter hormonal balances in such a way that it decreases lung cancer risk in women, but increases endometrial and ovarian cancer risk. We strongly encourage other research groups to examine the association between dietary acrylamide intake and the risk of lung cancer, separately among men and women and stratified by smoking status and alcohol consumption. 


\section{References}

1. Tareke E, Rydberg P, Karlsson P, Eriksson S, Tornqvist M. Analysis of acrylamide, a carcinogen formed in heated foodstuffs. J Agric Food Chem. 2002;50:4998-5006.

2. IARC. Monographs on the evaluation of carcinogen risk to humans: some industrial chemicals. Lyon: International Agency for Research on Cancer, 1994.

3. Cogliano V, Baan R, Straif K, et al. Future priorities for IARC Monographs. Lancet Oncol. 2008;9:708.

4. Hogervorst JG, Schouten LJ, Konings EJ, Goldbohm RA, van den Brandt PA. A Prospective Study of Dietary Acrylamide Intake and the Risk of Endometrial, Ovarian, and Breast Cancer. Cancer Epidemiol Biomarkers Prev. 2007;16:2304-2313.

5. Hogervorst JG, Schouten $L$, Konings EJ, Goldbohm RA, van den Brandt PA. Dietary acrylamide intake and the risk of renal cell, bladder, and prostate cancer. Am J Clin Nutr. 2008;87:1428-1438.

6. Olesen PT, Olsen A, Frandsen H, Frederiksen K, Overvad K, Tjonneland A. Acrylamide exposure and incidence of breast cancer among postmenopausal women in the Danish Diet, Cancer and Health Study. Int J Cancer. 2008;122:2094-2100.

7. Johnson KA, Gorzinski SJ, Bodner KM, et al. Chronic toxicity and oncogenicity study on acrylamide incorporated in the drinking water of Fischer 344 rats. Toxicol Appl Pharmacol. 1986;85:154-168.

8. Bull RJ, Robinson M, Laurie RD, et al. Carcinogenic effects of acrylamide in Sencar and A/J mice. Cancer Res. 1984;44:107-111.

9. Bull RJ, Robinson M, Stober JA. Carcinogenic activity of acrylamide in the skin and lung of Swiss-ICR mice. Cancer Lett. 1984;24:209-212.

10. Friedman MA, Dulak LH, Stedham MA. A lifetime oncogenicity study in rats with acrylamide. Fundam Appl Toxicol. 1995;27:95-105.

11. van den Brandt PA, Goldbohm RA, van 't Veer P, Volovics A, Hermus RJ, Sturmans F. A large-scale prospective cohort study on diet and cancer in The Netherlands. J Clin Epidemiol. 1990;43:285-295.

12. Barlow WE. Robust variance estimation for the case-cohort design. Biometrics. 1994;50:1064-1072.

13. Goldbohm RA, van den Brandt PA, Dorant E. Estimation of the coverage of Dutch municipalities by cancer registries and PALGA based on hospital discharge data. Tijdschr Soc Gezondheidsz. 1994;72:80-84.

14. Goldbohm RA, van den Brandt PA, Brants HA, et al. Validation of a dietary questionnaire used in a largescale prospective cohort study on diet and cancer. Eur J Clin Nutr. 1994;48:253-265.

15. Goldbohm RA, van ' $t$ Veer $P$, van den Brandt PA, et al. Reproducibility of a food frequency questionnaire and stability of dietary habits determined from five annually repeated measurements. Eur J Clin Nutr. 1995;49:420-429.

16 Cai J, Zeng D. Sample size/power calculation for case-cohort studies. Biometrics. 2004;60:1015-1024.

17. Konings EJ, Baars AJ, van Klaveren JD, et al. Acrylamide exposure from foods of the Dutch population and an assessment of the consequent risks. Food Chem Toxicol. 2003;41:1569-1579.

18. Rosen J, Hellenas KE. Analysis of acrylamide in cooked foods by liquid chromatography tandem mass spectrometry. Analyst. 2002;127:880-882.

19. Schettgen T, Rossbach B, Kutting B, Letzel S, Drexler H, Angerer J. Determination of haemoglobin adducts of acrylamide and glycidamide in smoking and non-smoking persons of the general population. Int J Hyg Environ Health. 2004;207:531-539.

20. Cox DR. Regression models and life-tables (with discussion). J R Statist Soc B. 1972;34:187-220.

21. Schoenfeld D. Partial residual for the proportional hazards regression model. Biometrika. 1982;69:239-241.

22.. Sunyer J, Kogevinas $M$, Kromhout $H$, et al. Pulmonary ventilatory defects and occupational exposures in a population-based study in Spain. Spanish Group of the European Community Respiratory Health Survey. Am J Respir Crit Care Med. 1998;157:512-517.

23. Ardies CM, Smith TJ, Kim S, Yang CS. Induction of 4-(methylnitrosamino)-1-(3-pyridyl)-1-butanone (NNK) activation in rat lung microsomes by chronic ethanol consumption and repeated running exercise. Cancer Lett. 1996;103:209-218.

24. Howard LA, Micu AL, Sellers EM, Tyndale RF. Low doses of nicotine and ethanol induce CYP2E1 and chlorzoxazone metabolism in rat liver. J Pharmacol Exp Ther. 2001;299:542-550.

25. Wang Z, Hall SD, Maya JF, Li L, Asghar A, Gorski JC. Diabetes mellitus increases the in vivo activity of cytochrome P450 2E1 in humans. Br J Clin Pharmacol. 2003;55:77-85.

26. Wauthier V, Verbeeck RK, Buc Calderon P. Age-related changes in the protein and mRNA levels of CYP2E1 and CYP3A isoforms as well as in their hepatic activities in Wistar rats. What role for oxidative stress? Arch Toxicol. 2004;78:131-138. 
27. Ghanayem BI, Witt KL, Kissling GE, Tice RR, Recio L. Absence of acrylamide-induced genotoxicity in CYP2E1null mice: evidence consistent with a glycidamide-mediated effect. Mutat Res. 2005;578:284-297.

28. Sobel W, Bond GG, Parsons TW, Brenner FE. Acrylamide cohort mortality study. Br J Ind Med. 1986;43:785788.

29. Marsh GM, Youk AO, Buchanich JM, Kant IJ, Swaen G. Mortality patterns among workers exposed to acrylamide: updated follow up. J Occup Environ Med. 2007;49:82-95.

30. Marsh GM, Lucas LJ, Youk AO, Schall LC. Mortality patterns among workers exposed to acrylamide: 1994 follow up. Occup Environ Med. 1999;56:181-190.

31. Exon JH. A review of the toxicology of acrylamide. J Toxicol Environ Health B Crit Rev. 2006;9:397-412.

32. Mucci LA, Adami HO. The role of epidemiology in understanding the relationship between dietary acrylamide and cancer risk in humans. Adv Exp Med Biol. 2005;561:39-47.

33. Segerback D, Calleman CJ, Schroeder JL, Costa LG, Faustman EM. Formation of N-7-(2-carbamoyl-2hydroxyethyl)guanine in DNA of the mouse and the rat following intraperitoneal administration of [14C]acrylamide. Carcinogenesis. 1995;16:1161-1165.

34. Ali SF, Hong JS, Wilson WE, Uphouse LL, Bondy SC. Effect of acrylamide on neurotransmitter metabolism and neuropeptide levels in several brain regions and upon circulating hormones. Arch Toxicol. 1983;52:3543.

35. Mannaa F, Abdel-Wahhab MA, Ahmed HH, Park MH. Protective role of Panax ginseng extract standardized with ginsenoside Rg3 against acrylamide-induced neurotoxicity in rats. J Appl Toxicol. 2006;26:198-206.

36. Uphouse LL, Nemeroff CB, Mason G, Prange AJ, Bondy SC. Interactions between "handling" and acrylamide on endocrine responses in rats. Neurotoxicology. 1982;3:121-125.

37. Bowyer JF, Latendresse JR, Delongchamp RR, et al. The effects of subchronic acrylamide exposure on gene expression, neurochemistry, hormones, and histopathology in the hypothalamus-pituitary-thyroid axis of male Fischer 344 rats. Toxicol Appl Pharmacol. 2008;230:208-215.

38. Clement FC, Dip R, Naegeli H. Expression profile of human cells in culture exposed to glycidamide, a reactive metabolite of the heat-induced food carcinogen acrylamide. Toxicology. 2007;240:111-124.

39. Harris HA, Katzenellenbogen JA, Katzenellenbogen BS. Characterization of the biological roles of the estrogen receptors, ERalpha and ERbeta, in estrogen target tissues in vivo through the use of an ERalphaselective ligand. Endocrinology. 2002;143:4172-4177.

40. Ishibashi H, Suzuki T, Suzuki S, et al. Progesterone receptor in non-small cell lung cancer--a potent prognostic factor and possible target for endocrine therapy. Cancer Res. 2005;65:6450-6458.

41. Lin P, Chang JT, Ko JL, Liao SH, Lo WS. Reduction of androgen receptor expression by benzo[alpha]pyrene and 7,8-dihydro-9,10-epoxy-7,8,9,10-tetrahydrobenzo[alpha]pyrene in human lung cells. Biochem Pharmacol. 2004;67:1523-1530.

42. Omoto $Y$, Kobayashi $Y$, Nishida K, et al. Expression, function, and clinical implications of the estrogen receptor beta in human lung cancers. Biochem Biophys Res Commun. 2001;285:340-347.

43. Rodriguez C, Spencer Feigelson H, Deka A, et al. Postmenopausal hormone therapy and lung cancer risk in the cancer prevention study II nutrition cohort. Cancer Epidemiol Biomarkers Prev. 2008;17:655-660.

44. Kabat GC, Miller AB, Rohan TE. Reproductive and hormonal factors and risk of lung cancer in women: a prospective cohort study. Int J Cancer. 2007;120:2214-2220. 



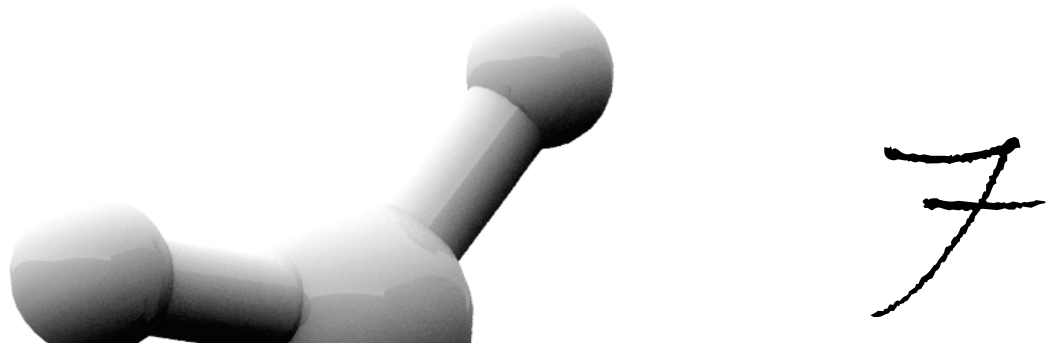

\section{Dietary acrylamide intake and brain cancer risk}

Janneke G.F. Hogervorst

Leo J. Schouten

Erik J.M. Konings

R. Alexandra Goldbohm

Piet A. van den Brandt 


\begin{abstract}
Background Acrylamide is a probable human carcinogen, which is present in several heattreated foods. In epidemiological studies, positive associations with endometrial, ovarian, and renal cell cancer risk have been observed. The incidence of central nervous system tumours was increased upon acrylamide administration in drinking water to rats. In the current study, the association between dietary acrylamide intake and human brain cancer risk was investigated for the first time.
\end{abstract}

Methods In 1986, 120,852 persons (ages 55-69 years) were included in the Netherlands Cohort Study on diet and cancer. At baseline, a random subcohort of 5000 participants was selected from the total cohort for a case-cohort approach. Acrylamide intake was assessed with a food frequency questionnaire at baseline and based on acrylamide analyses in relevant Dutch foods. Hazard ratios (HRs) were calculated using Cox proportional hazards analysis. Subgroup analyses were done for microscopically verified brain cancer, astrocytic gliomas, high-grade astrocytic gliomas, and never-smokers. The acrylamide risk estimates were adjusted for possible brain cancer risk factors.

Results After 16.3 years of follow-up, 216 brain cancer cases were available for analysis. The multivariable-adjusted HR per $10 \mu \mathrm{g} /$ day increment of acrylamide intake was 1.02 (95\% confidence interval: 0.89-1.16). HRs were not significantly increased either when dietary acrylamide intake was analysed as a categorical variable. Also, there was no association in the subgroups based on histology and smoking.

Conclusion In this prospective cohort study, acrylamide intake was not associated with brain cancer risk. 


\section{Introduction}

Acrylamide has been classified as a probable human carcinogen based on animal studies (1), and is since 2002 known to be present in several carbohydrate-rich heated foods, such as French fries and potato crisps. Recent epidemiological studies on dietary acrylamide intake and cancer risk reported positive associations with endometrial, ovarian, and renal cell cancer risk $(2,3)$. An advisory group of the IARC recently gave high priority to acrylamide for assessment in future IARC Monograph series (4).

Because the acrylamide molecule is small and hydrophilic, it passively diffuses throughout the whole body and for this reason, theoretically all tissues are targets for acrylamide carcinogenesis. The incidence of central nervous system tumours was increased upon acrylamide administration in drinking water in one of the two lifetime carcinogenicity studies with rats $(5,6)$. In this prospective cohort study, we investigated the association between dietary acrylamide intake and human brain cancer risk.

\section{Methods}

\section{Study participants}

The study took place within the Netherlands Cohort Study on diet and cancer, which started in September 1986 (7). At baseline, the participants (58,279 men and 62,573 women aged 55-69 years) completed a self-administered questionnaire on diet and other possible risk factors for cancer. The case-cohort approach was used for data processing and analysis; cases were enumerated for the entire cohort, whereas the accumulated person-time for the total cohort was estimated from a subcohort of 5000 men and women randomly sampled from the full cohort at baseline. Cases and subcohort members were excluded from the analysis if they had been diagnosed with cancer (other than skin cancer) at baseline and if their dietary data were incomplete or inconsistent.

\section{Follow-up}

Incident cases in the total cohort were detected by record linkage to the Netherlands Cancer Registry. The completeness of cancer follow-up through linkage with these cancer registries was assessed to be at least $96 \%$, whereas the follow-up of the subcohort at the end of the follow-up period was nearly $100 \%$ complete. Further details on the design of the study and methods of follow-up are presented elsewhere (7).

\section{Acrylamide intake assessment}

The Netherlands Cohort Study on diet and cancer food frequency questionnaire contained questions on habitual consumption of 150 food items during the year preceding the baseline. We used data on acrylamide concentrations in foods on the Dutch market to obtain an intake estimate representative for the Dutch situation. The acrylamide intake was estimated from the mean acrylamide level of foods, and the frequency of consumption and portion size of the foods. For further details of the intake assessment, see (2). 


\section{Statistical analysis}

Hazard ratios (HRs) were obtained through Cox proportional hazards regression with STATA software (package 9.2). Smoking does not seem to be a clear risk factor for brain cancer in adults (8), but smokers have on average three to four times higher concentrations of acrylamide-haemoglobin adducts (a marker of internal doses of acrylamide) compared with non-smokers (9). Therefore, subgroup analyses were done for neversmokers. Other analyses were based on histological subtype: astrocytic glioma (ICD-O morphology codes: M9384, M9400-9401, M9410-9411, M9420-9421, M9424, M94409442, M9481; other case groups were too small for meaningful analysis), and astrocytic glioma with a high malignancy grade (M9400-9401 differentiation grade 3-4, M9410-9411 differentiation grade 3-4, M9420 differentiation grade 3-4, M9424 differentiation grade 34, M9440-9442, M9481) (10).

The HRs for acrylamide intake were adjusted for sex, age and the a priori selected covariables smoking (status, quantity and duration), body mass index, height, education level, and energy intake. The other variables from Table 1 were checked for confounding potential based on causing a change of $>10 \%$ in the HR of acrylamide and incorporated as covariables if they fulfilled this criterion. We considered 100 cases as the minimum number needed for quintile analyses, 60 for tertile analyses and 20 for analyses with acrylamide as a continuous variable.

\section{Results}

After 16.3 years of follow-up, there were 259 cases of primary brain cancer (ICD-O-3: C71), of which 205 were microscopically verified. One hundred and eighty cases were of the astrocytic glioma type, and there were 158 cases with high-grade astrocytic glioma. Table 1 shows the characteristics of the subcohort and the brain cancer cases. Cases consumed more alcohol, were more physically active, smoked more and longer, had a somewhat higher education level and body mass index, and were slightly taller than the subcohort members. There was no effect modification by sex, and therefore the association between acrylamide intake and brain cancer risk is shown for both sexes combined.

Acrylamide intake was not associated with brain cancer risk (Table 2), either adjusted only for sex and age or multivariable-adjusted. The same applied to microscopically verified brain cancer, astrocytic glioma, and high-grade astrocytic glioma. Also in the never-smokers, no association was found. The most important acrylamide-contributing foods in this cohort (Dutch spiced cake and coffee) were by themselves also not associated with brain cancer risk (results not shown).

\section{Discussion}

This prospective cohort study does not give support for an association between dietary acrylamide intake and human brain cancer risk, which was not studied before. 


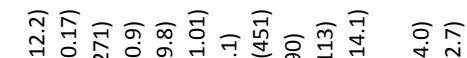

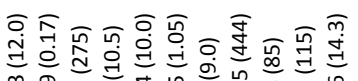

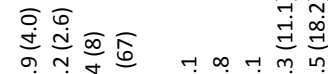

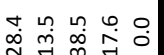

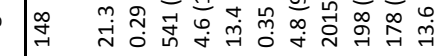

它婇吉吉

लें

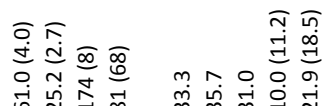

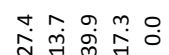

㲾

$0 \wedge \rightarrow \infty$

ले भिं लें

कำ

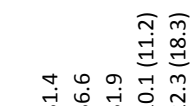

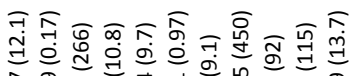

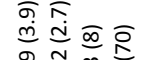

芒

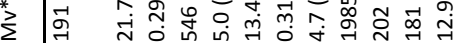

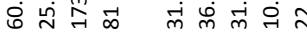

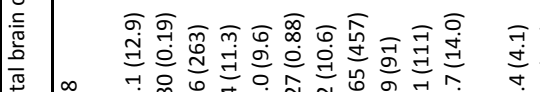

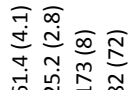

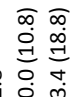

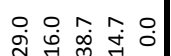
$\dot{m} \dot{m} \dot{m} \dot{m} \dot{\sim} \dot{\sim}$

a$$
\frac{n}{\frac{n}{0}}
$$

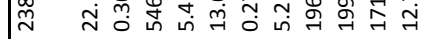
엑

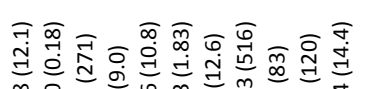

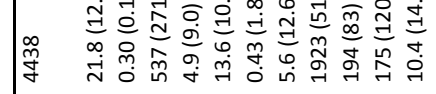

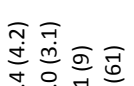

åd

में

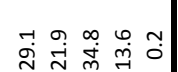

列

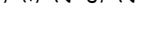




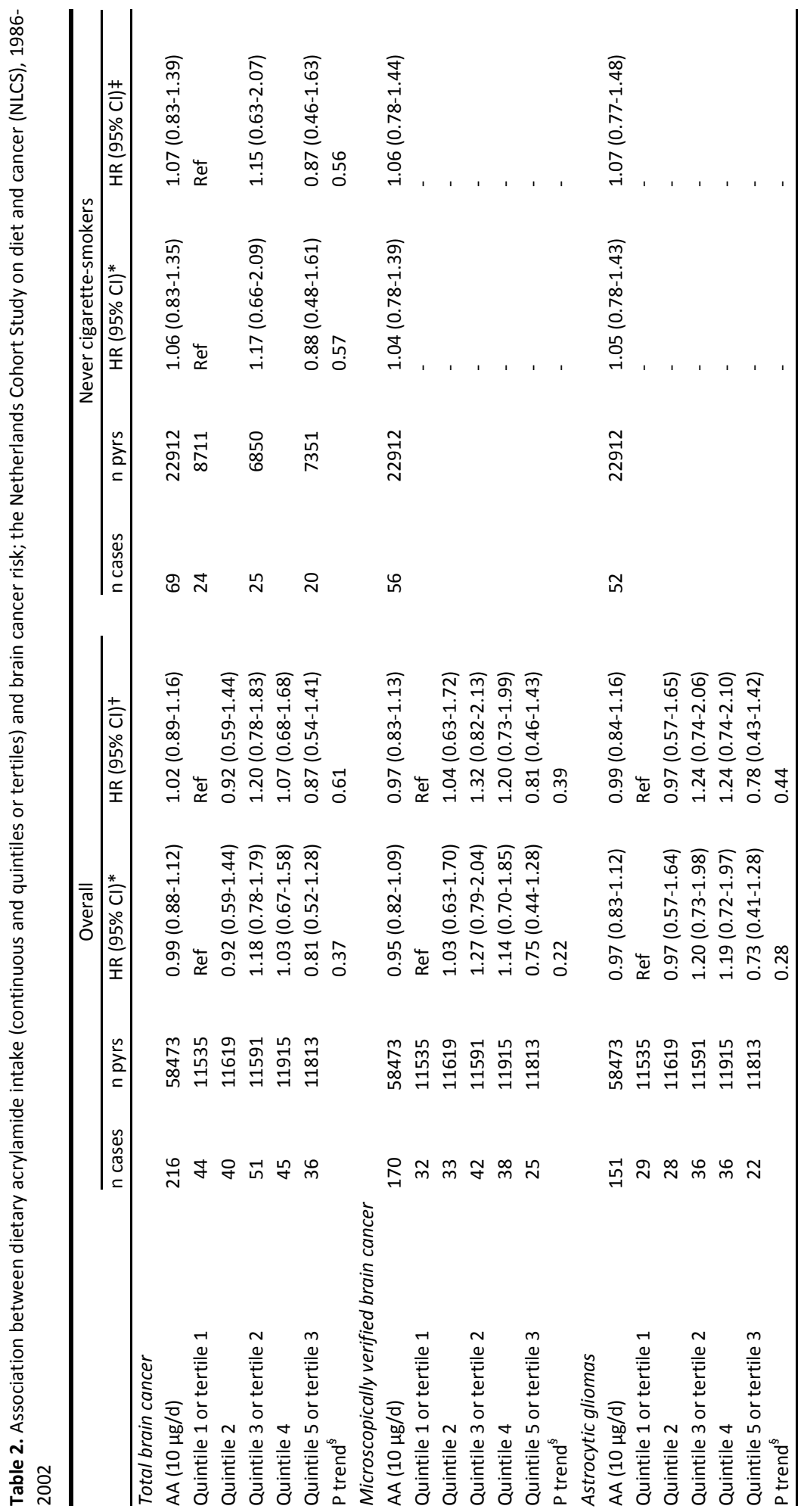




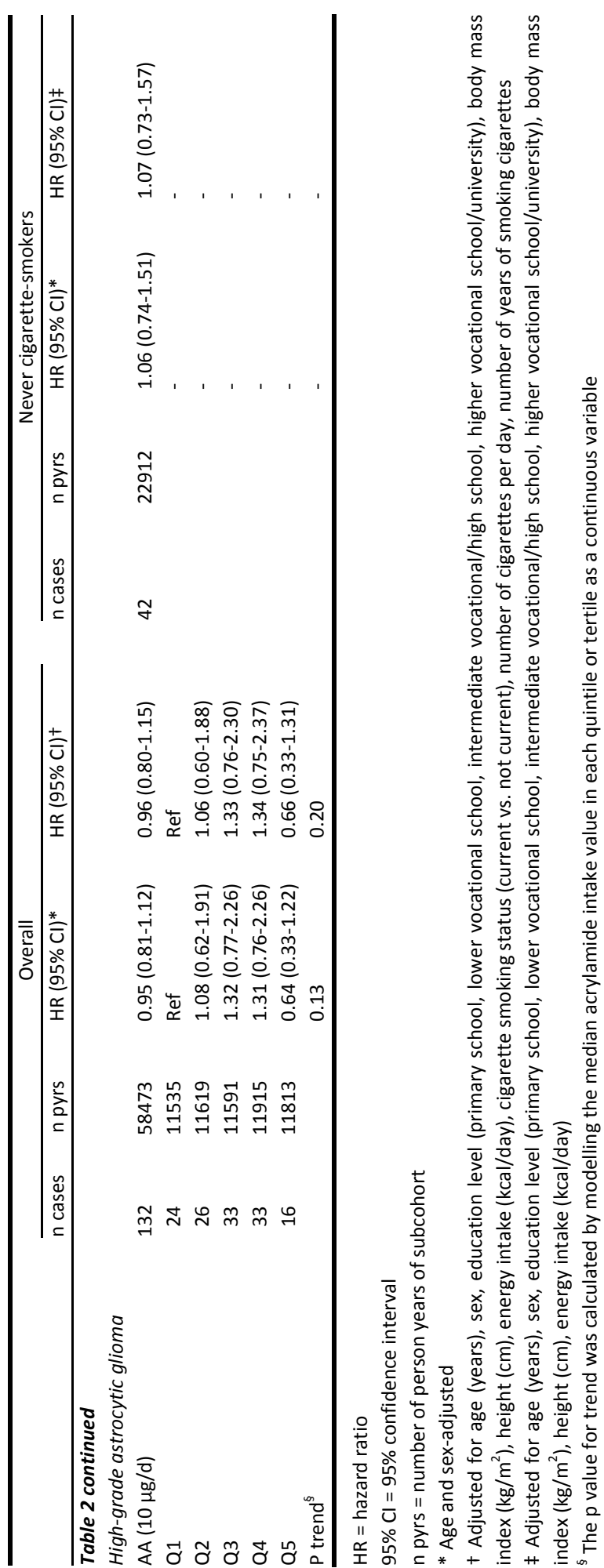


Although the route of exposure differs, these results are in line with studies on occupational acrylamide exposure, which did not show excess brain cancer incidence in association with acrylamide exposure $(11,12)$. The number of cases in the analyses was modest, especially in the subgroups based on histology. According to the formula of Cai and Zeng (13) for case-cohort studies, the power was $80 \%$ to detect a statistically significant (significance level of 0.05 ) association between acrylamide intake (highest versus lowest quintile) and brain cancer risk of 1.85 or higher.

The strengths of this prospective study include the absence of recall bias and selection bias, and the fact that only acrylamide data for foods from the Dutch market were used (2). Also, assigning mean acrylamide concentrations to individual foods that are known to have considerable variation in acrylamide concentrations was shown to result in a reasonable rank ordering of the acrylamide intake of the participants in a 24-hour duplicate diet study (2). Despite the potential of non-differential misclassification of the acrylamide intake (for a discussion of this issue, see Hogervorst et al., (2)), we observed statistically significant positive associations between acrylamide intake and endometrial, ovarian and renal cell cancer risk in previous analyses in the Netherlands Cohort Study on diet and cancer $(2,3)$.

In conclusion, this prospective study does not give support for an association between dietary acrylamide intake and brain cancer risk. 


\section{References}

1. IARC. Monographs on the evaluation of carcinogen risk to humans: some industrial chemicals. Lyon: International Agency for Research on Cancer, 1994.

2. Hogervorst JG, Schouten LJ, Konings EJ, Goldbohm RA, van den Brandt PA. A Prospective Study of Dietary Acrylamide Intake and the Risk of Endometrial, Ovarian, and Breast Cancer. Cancer Epidemiol Biomarkers Prev 2007;16:2304-2313.

3. Hogervorst JG, Schouten LJ, Konings EJ, Goldbohm RA, van den Brandt PA. Dietary acrylamide intake and the risk of renal cell, bladder, and prostate cancer. Am J Clin Nutr 2008;87:1428-38.

4. Cogliano V, Baan R, Straif K, et al. Future priorities for IARC Monographs. Lancet Oncol 2008;9:708.

5. Johnson KA, Gorzinski SJ, Bodner KM, et al. Chronic toxicity and oncogenicity study on acrylamide incorporated in the drinking water of Fischer 344 rats. Toxicol Appl Pharmacol 1986;85:154-68.

6. Friedman MA, Dulak LH, Stedham MA. A lifetime oncogenicity study in rats with acrylamide. Fundam Appl Toxicol 1995;27:95-105.

7. van den Brandt PA, Goldbohm RA, van ' $t$ Veer P, Volovics A, Hermus RJ, Sturmans F. A large-scale prospective cohort study on diet and cancer in The Netherlands. J Clin Epidemiol 1990;43:285-95.

8. Ohgaki H, Kleihues P. Epidemiology and etiology of gliomas. Acta Neuropathol 2005;109:93-108.

9. Vesper HW, Slimani N, Hallmans G, et al. Cross-sectional study on acrylamide hemoglobin adducts in subpopulations from the European Prospective Investigation into Cancer and Nutrition (EPIC) Study. J Agric Food Chem 2008;56:6046-53.

10. van der Sanden GA, Wesseling P, Schouten LJ, Teepen HL, Coebergh J. A uniform histological cluster scheme for ICD-O-coded primary central nervous system tumors. Neuroepidemiology 1998;17:233-46.

11. Swaen GM, Haidar S, Burns CJ, et al. Mortality study update of acrylamide workers. Occup Environ Med 2007; 64:396-401.

12. Marsh GM, Youk AO, Buchanich JM, Kant IJ, Swaen G. Mortality patterns among workers exposed to acrylamide: updated follow up. J Occup Environ Med 2007;49:82-95.

13. Cai J, Zeng D. Sample size/power calculation for case-cohort studies. Biometrics 2004;60:1015-24. 



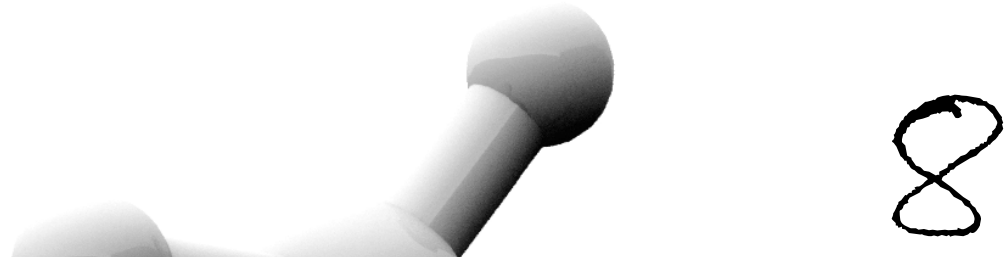

Dietary acrylamide intake and the risk of head-neck and thyroid cancer: results from the Netherlands Cohort Study

Leo J. Schouten Janneke G.F. Hogervorst

Erik J.M. Konings

R. Alexandra Goldbohm

Piet A. van den Brandt 


\begin{abstract}
Acrylamide exposure led to an increased incidence of oral and thyroid tumours in animal studies. In 1986, 120,852 persons (aged 55-69 years) were included in the Netherlands Cohort Study. Dietary acrylamide intake was assessed with a food-frequency questionnaire and was based on chemical analysis of all relevant Dutch foods. Hazard ratios (HRs) were adjusted for smoking and other confounders. After 16.3 years of follow-up, there were 101, 83, 180 and 66 cases of oral cavity, oro-hypopharynx, larynx and thyroid cancer, respectively. The average daily dietary acrylamide intake was $21.8 \mu \mathrm{g}$ (standard deviation 12.1). Dietary acrylamide intake was not associated with an increased risk of oral cavity cancer (HR, 95\% confidence interval (Cl) per $10 \mu \mathrm{g}$ intake/day: 0.90, 0.73-1.10), orohypopharynx cancer (HR [Cl]: 0.74, 0.53-1.03), larynx cancer (HR [Cl]: 1.05, 0.91-1.21), and thyroid cancer (HR [Cl]: 1.03, 0.82-1.27). In non-smoking participants, HRs were not increased either. Dietary acrylamide was statistically significantly associated with an increased risk of oral cavity cancer in non-smoking women, but case numbers were small. Dietary acrylamide intake was not positively associated with the risk of head-neck and thyroid cancer, except with oral cavity cancer risk in non-smoking women. There were indications for a negative association in men.
\end{abstract}




\section{Introduction}

Acrylamide is classified as a probable human carcinogen based on animal studies and mechanistic insights (1). Animal studies have shown positive dose-response relationships between acrylamide exposure and cancer in multiple organs and tissues in rodents, among which were the mammary gland, skin, lungs, oral tissues and thyroid gland (2-5). Acrylamide was administered through gastric intubation, intraperitoneal injection and dermal application $(2,3)$ or the drinking water $(4,5)$ to the animals at high doses. The discovery in 2002 that acrylamide is present in many foods, such as French fries and potato crisps, gave therefore rise to substantial concern (6).

In occupational studies, no association was observed between exposure to acrylamide and the risk of head-neck cancer. The incidence of thyroid cancer was increased, but the number of cases was small $(7,8)$. Exposure to dietary acrylamide and the risk of cancers of the oral cavity, pharynx and larynx has been investigated in a hospital-based case-control study and no association was observed (9).

Recently, three prospective epidemiological studies demonstrated a positive association between dietary acrylamide exposure and risk of cancer. Positive associations were observed for endometrial, ovarian (10), and renal cell cancer (11), and for postmenopausal estrogen receptor-positive breast cancer (12). Because the acrylamide molecule is small and hydrophilic, it reaches every organ and virtually every tissue in the body $(13,14)$. For this reason, theoretically all organs and tissues are targets for carcinogenesis. Considering the positive associations observed in animal experiments and the limited evidence available for humans, we investigated the association between dietary acrylamide intake and the risk of head-neck and thyroid cancers in a large prospective cohort study.

\section{Materials and methods}

\section{Study design and participants}

The study took place within the prospective Netherlands Cohort Study on diet and cancer (NLCS), which started in September 1986 with the inclusion of 58,279 men and 62,573 women aged 55-69 years sampled from Dutch municipal population registries (15). At baseline, the participants completed a self-administered questionnaire on diet and other risk factors for cancer, such as smoking, anthropometry and physical activity. A casecohort approach (16) was used for data processing and analysis; cases were enumerated for the entire cohort, whereas the accumulated person-years for the total cohort were estimated from a subcohort of $\mathbf{5 0 0 0}$ men and women randomly sampled from the full cohort at baseline. Incident cases in the total cohort have been detected by annual computerised record linkages to the Netherlands Cancer Registry and the Netherlands Pathology Registry (PALGA). The completeness of cancer follow-up through linkage with these cancer registries was assessed to be at least $96 \%$ (17), whereas the follow-up of the subcohort at the end of the follow-up period was nearly $100 \%$ complete (only one male 
subcohort member was lost to follow-up). Further details on the design of the study are presented elsewhere $(15,18-20)$.

After 16.3 years of follow-up (Sept. 1986-Dec. 2002), there were 467 cases of histologically verified head-neck cancer (ICD-O-3: C00.3-C06.9; C09.0-C10.9; C12.0-C14.9 and C32) and 73 cases of histologically verified thyroid cancer (ICD-O-3: C73). Because of the small numbers and the different etiology (21), cases with salivary gland cancer $(n=41)$, nasopharyngeal cancer $(n=18)$ or cancer of the nasal cavity and sinuses $(n=40)$ were not included in the analysis. After exclusion of non-squamous cell carcinomas and using the grouping proposed by Hashibe et al. (22), there were 434 cohort members with a headneck squamous cell cancer, including 116 cancers of the oral cavity, 108 cancers of oropharynx and hypopharynx, 227 cancers of the larynx, and 3 cancers of an unspecified location within the head-neck region. Several cases had more than one primary cancer in the head-neck area (three cases with three cancers and nine cases with two cancers each) and each primary cancer within a site was regarded as an endpoint in site-specific analyses.

Cases and subcohort members were excluded from the analysis if they had been diagnosed to have cancer (other than skin cancer) at baseline, if their dietary data were incomplete or inconsistent and if data on confounders were missing. Figure 1 shows the selection and exclusion steps that resulted in the numbers of cases and subcohort members that were available for analysis.

\section{Dietary acrylamide intake assessment}

The NLCS food frequency questionnaire (FFQ) contained questions on 150 food items (18). It queried the habitual intake, both frequency and portion size, of foods during the year preceding baseline.

The major dietary sources of acrylamide in the Netherlands in 1998 (determined through a National Food Consumption Survey) were estimated to be potato crisps (31\%), French fries (21\%), Dutch spiced cake (16\%), coffee (13\%), bread (10\%), and cookies (4\%) (23). Although relative contributions may have changed between 1986 (start NLCS) and 1998 , the major sources have probably stayed the same. The mentioned sources were all included as separate items in the NLCS FFQ, and thus the most important dietary acrylamide sources in the Netherlands were taken into account in the exposure assessment. We used data on acrylamide concentrations in food products on the Dutch market in order to obtain an intake estimate representative for the Dutch situation. For each food item, the contribution to dietary acrylamide intake was estimated from the mean acrylamide level in the food, its consumption frequency and portion size. Further details of the dietary acrylamide intake assessment, including concentrations of acrylamide in the foods that were used in the intake calculation, can be found elsewhere (10).

\section{Statistical analysis}

Confounders were selected in two steps: some were chosen a priori to be included in the models, and other potential confounders were only included if they changed age- and sexadjusted hazard ratios of dietary acrylamide (expressed as the interval between the 10th 
and 90th percentile of intake of the subcohort: $27 \mu \mathrm{g}$ acrylamide/day) by more than ten percent. The a priori selected covariables were age, sex, smoking status (current vs. not current), number of cigarettes/day, and number of smoking years. The following variables were checked for confounding: body mass index, height, energy intake, consumption of vegetables, fruits, dairy, meat, processed meat, fish and alcohol, non-occupational physical activity, smoking of cigars or pipe (current, former or never), educational level, and intake of vitamin $A$ and $C, \alpha$ - and $\beta$-carotene, folate, niacin, fat and saturated fat, trans fatty acid, carbohydrates, and fibre, and use of vitamin A supplements. Smokers have on average three to four times higher levels of acrylamide-haemoglobin adducts (which is a marker of internal dose of acrylamide) than non-smokers (24). For this reason and because of the fact that smoking is such an important risk factor for head-neck cancers, subgroup analyses were done for non-smokers (never-smokers combined with former smokers who had quit smoking more than ten years before baseline).

$102,852 \mathrm{M}+\mathrm{F}$

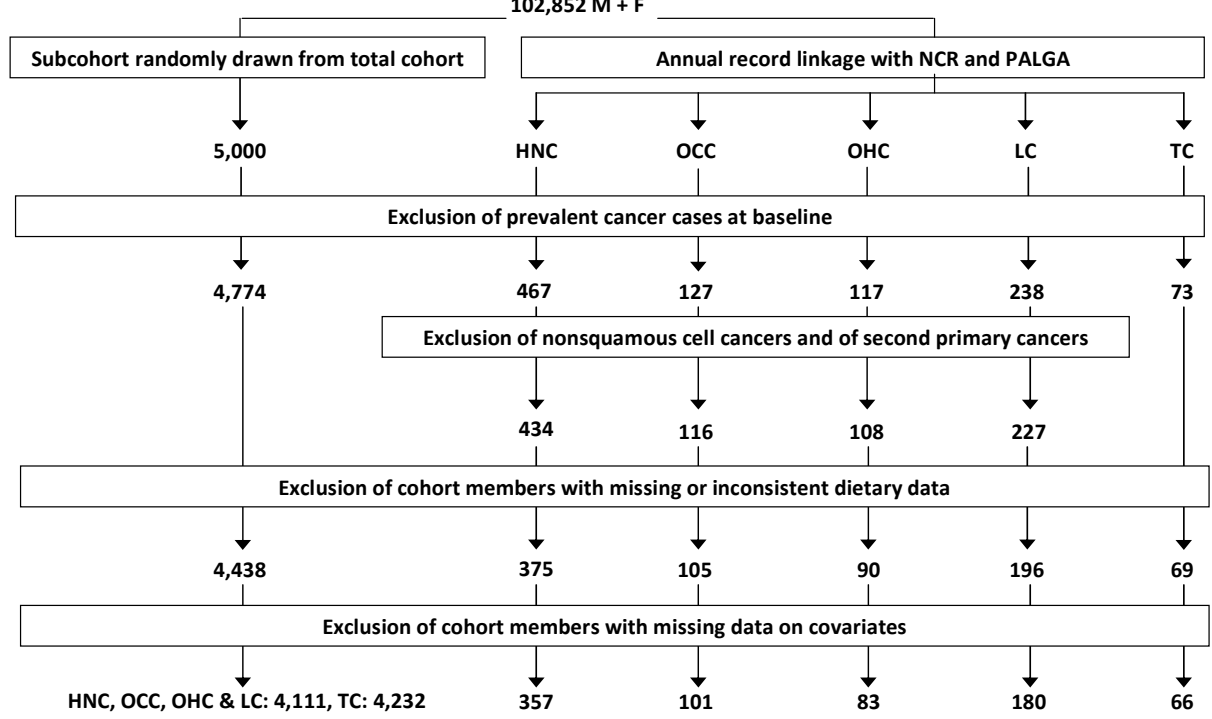

Figure 1: Flow diagram of subcohort members and cases on whom the analyses were based; Netherlands Cohort Study on diet and cancer, 1986-2002

HNC, Head-neck cancer; LC, Larynx cancer; NCR, Netherlands Cancer Registry; OCC, Oral Cavity Cancer; OHC, Oropharynx-hypopharynx cancer; PALGA, Netherlands Pathology Register; TC, Thyroid Cancer.

To investigate if the observed associations could be attributed to dietary acrylamide specifically or whether the association was due to other characteristics of acrylamidecontaining foods, models were run with dietary acrylamide (as continuous variable) and the foods Dutch spiced cake and coffee (one at a time) that explained most variance in dietary acrylamide intake. 
Scaled Schoenfeld residuals were used to test the proportional hazards assumption (25). When according to this test the proportional hazards assumption might be violated, the hazard curves were inspected visually. Hazard ratios (HRs) and confidence intervals (Cl) were obtained through Cox proportional hazards regression (26) with STATA software (package 9.2), with person time on study as the time metric. Additional variance introduced by sampling a subcohort from the cohort was taken into account by estimation of standard errors using the robust Huber-White sandwich estimator, a method similar to the variance-covariance estimator by Barlow (27). Tests for dose-response trends were done by fitting the median dietary acrylamide intake per quintile or tertile as a continuous variable. We applied 20 cases per category as the minimum number needed to do the Cox regression analyses. To check for the influence that preclinical disease may have had on the results, the analyses were also done with exclusion of the first two years of follow-up.

\section{Results}

The average daily dietary acrylamide intake of the subcohort was $21.8 \mu \mathrm{g}$ (standard deviation (SD: 12.1) in all participants; $22.5 \mu \mathrm{g}$ (SD: 12.1) in men and $21.0 \mu \mathrm{g}$ (SD: 11.9) in women. As described elsewhere (11), the most important source of dietary acrylamide in the subcohort was coffee, which contributed on average $47 \%$ to the dietary acrylamide intake, whereas Dutch spiced cake, cookies, French fries, and potato crisps contributed 15, 13,8 , and $2 \%$, respectively. However, most of the variance of the dietary acrylamide intake was explained by Dutch spiced cake (57\%), followed by coffee (15\%), French fries (14\%), potato crisps (5\%), and cookies (3\%).

Male cases with head-neck tumours were slightly older at baseline than the subcohort members were, especially cases with oro- and hypopharynx cancer (Table 1). Overall, female cases with oral cavity cancer were older and cases with oro- and hypopharynx cancer were younger than the subcohort members were (Table 2). In both men and women, fruit intake was lower in cases than in subcohort members and alcohol intake was higher. Cases were also more often current smokers, smoked more cigarettes per day and had smoked longer than subcohort members had. Thyroid cancer cases had lower intake of dairy products, alcohol and coffee compared with the subcohort. More often than subcohort members, thyroid cancer cases were non- and never-smokers.

In multivariable-adjusted analyses, dietary acrylamide intake was associated with decreased risks of head-neck cancer, men and women combined, in all quintiles (compared with the lowest quintile of intake), although only the HR in the second quintile of intake was statistically significantly decreased (see Table 3 ). There was no further decreasing risk with increasing dietary acrylamide intake, and the $p$ for trend was 0.40 . HRs in men were not very different from the overall results, but the multivariable-adjusted HRs in women were closer to unity. When the analysis was restricted to non-smokers (neversmokers and smokers who quitted smoking $\geq 10$ years before baseline), HRs for head-neck cancer in the highest tertile of intake remained lower than unity in the analyses for men and women combined and men alone. The HR for those in the highest tertile of intake in 
non-smoking men was statistically significantly decreased (HR: $0.45 ; 95 \% \mathrm{Cl}: 0.21-0.94)$, and the $p$ for trend was 0.03 . HRs for continuous intake were also decreased, but not statistically significant. The HRs for dietary acrylamide intake did not change notably when Dutch spiced cake or coffee were added to the multivariable model (results not shown). The results did not change importantly when the first two years of follow-up were excluded (results not shown).

Next, analyses were conducted for different subsites of head-neck cancer: oral cavity, oro- and hypopharynx and larynx cancers. Multivariable-adjusted analyses for oral cavity cancer (see Table 4) and oro- and hypopharynx cancers (see Table 5) yielded comparable results as the analyses for head-neck cancer overall: most HRs were decreased. For oral cavity cancer, most HRs were decreased. Because of the low number of non-smoking cases, only an analysis with dietary acrylamide as a continuous exposure variable was conducted in this subgroup, showing no association in men and women combined and an increased multivariable-adjusted $\mathrm{HR}$ for oral cavity cancer in women (HR $1.28 ; 95 \% \mathrm{Cl}$ : 1.01-1.62 per $10 \mu \mathrm{g}$ intake per day). For oro- and hypopharynx cancers, HRs were decreased. The multivariable-adjusted HR for all men per $10 \mu \mathrm{g}$ of daily intake was 0.68 (95\% $\mathrm{Cl}$ : 0.42-1.00). For women, HRs were closer to unity than for men.

Unlike oral cavity and oro- and hypopharynx cancers, HRs for the association between dietary acrylamide and risk of larynx cancer were for the larger part close to or higher than 1 (see Table 6). Only the HRs for continuous intake in non-smokers were statistically nonsignificantly decreased: 0.82 (95\% Cl: $0.53-1.29)$ in men and women combined and 0.85 (95\% Cl: 0.59-1.34) in men. The HRs for dietary acrylamide intake did not change importantly when coffee was added to the model, but HRs did change considerably when Dutch spiced cake was added to the model. HRs increased strongly for oral cavity in non-smoking women (HR: 1.67 [95\% Cl: 0.62-4.49] per $10 \mu \mathrm{g} /$ day) and laryngeal cancer in non-smoking men (HR: 1.14 [95\% Cl: 0.46-2.81] per $10 \mu \mathrm{g} /$ day). When the first two years of follow-up were excluded, the results were essentially the same (results not shown).

Multivariable-adjusted HRs for thyroid cancer were all close to unity (see Table 7), although the HR for the highest tertile of intake (compared with the lowest tertile) in all men and women was slightly increased: 1.33 (95\% Cl: 0.70-2.53). The $\mathrm{p}$ for trend was 0.42 . When analyses were restricted to the follicular subtype of thyroid cancer (30 cases), the multivariable adjusted HR in both sexes for continuous intake per $10 \mu \mathrm{g} /$ day was 0.91 (95\% Cl: 0.69-1.19). The results did not change when coffee was added to the model, but HRs increased considerably when Dutch spiced cake was added to the model. Especially in the models with non-smokers only -with a low number of cases- the change in HR was large. The results did not change importantly when the first two years of follow-up were excluded (results not shown).

\section{Discussion}

In this large prospective cohort study, we did not observe a positive association between the intake of dietary acrylamide and the risk of head-neck and thyroid cancers in men. 


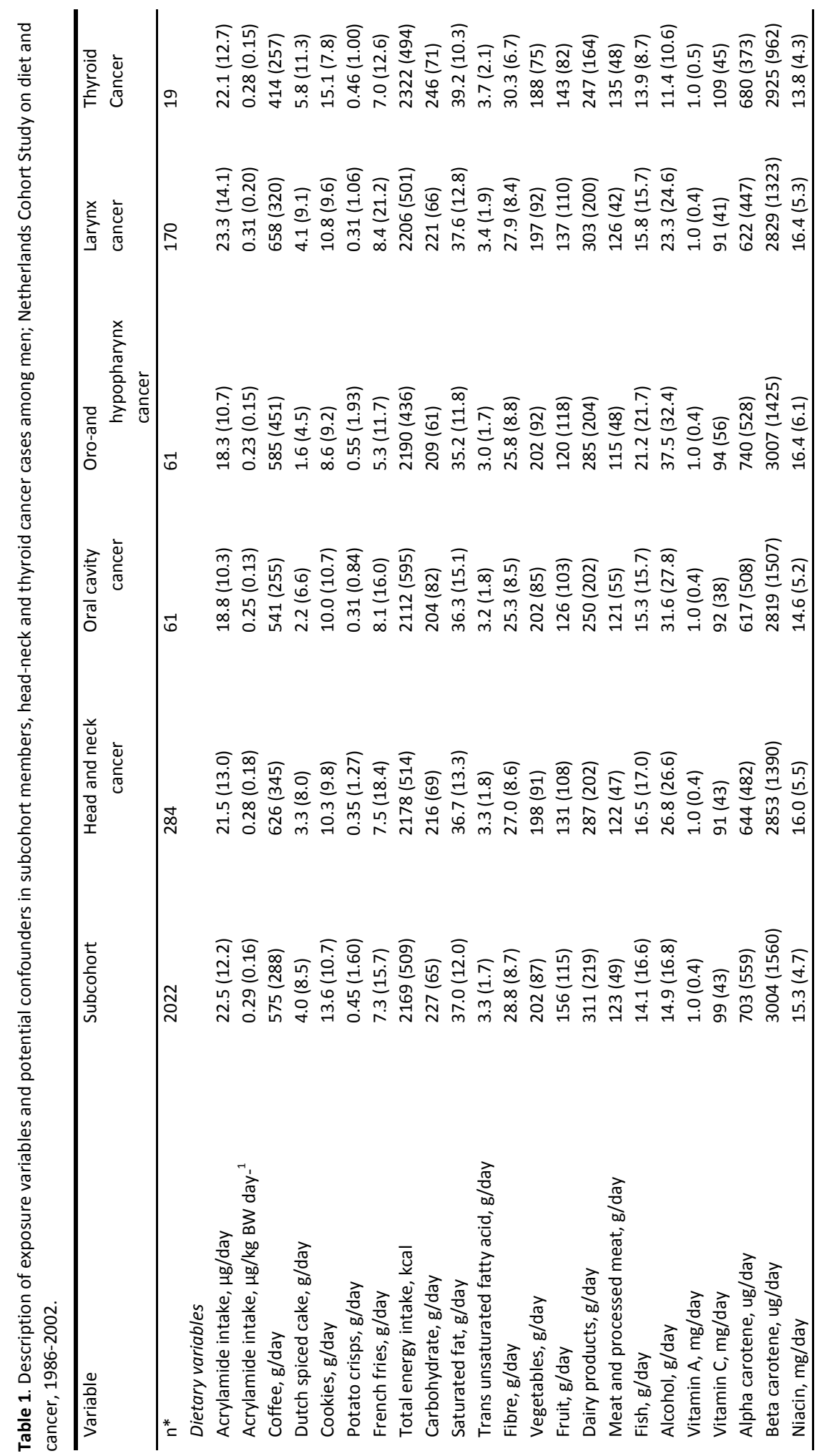




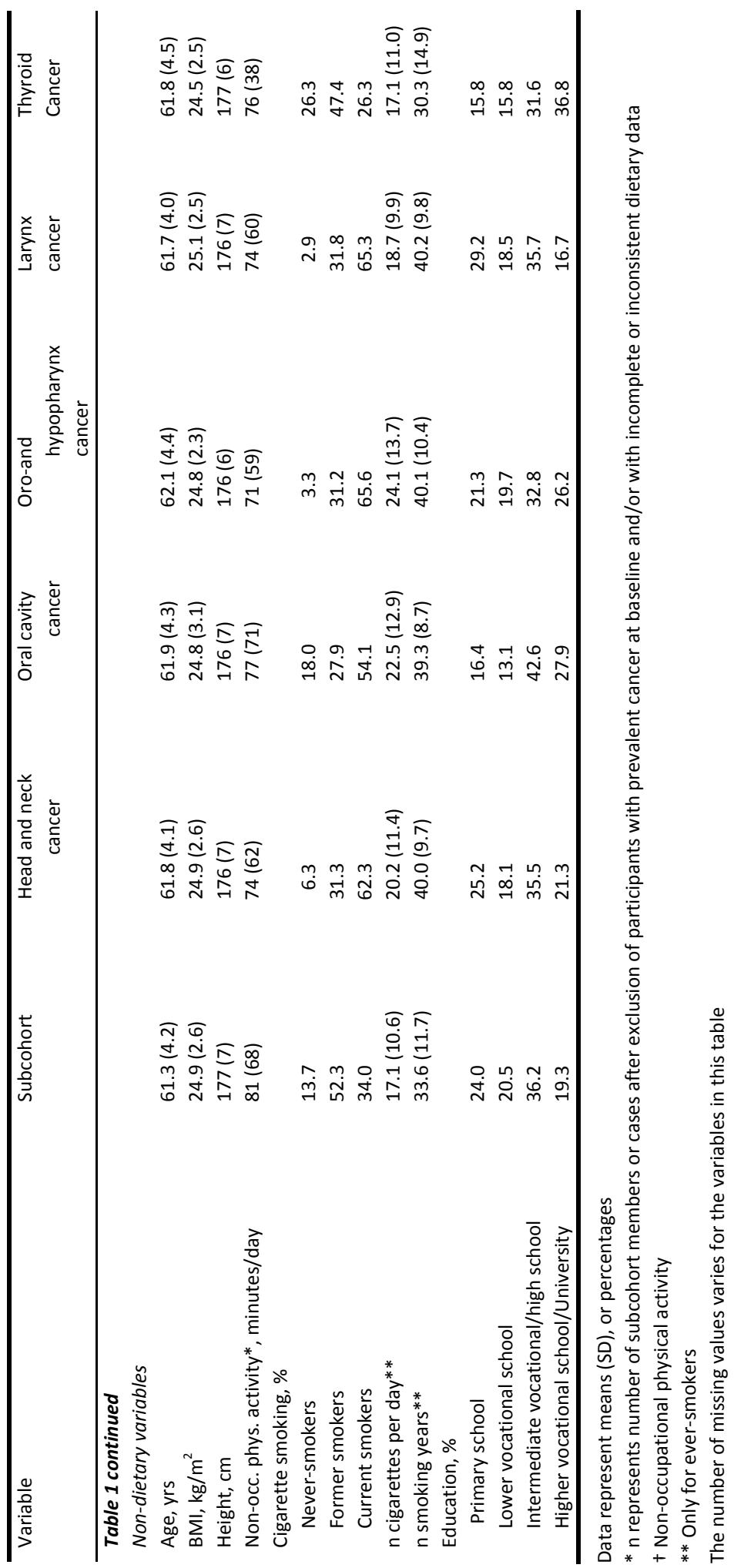




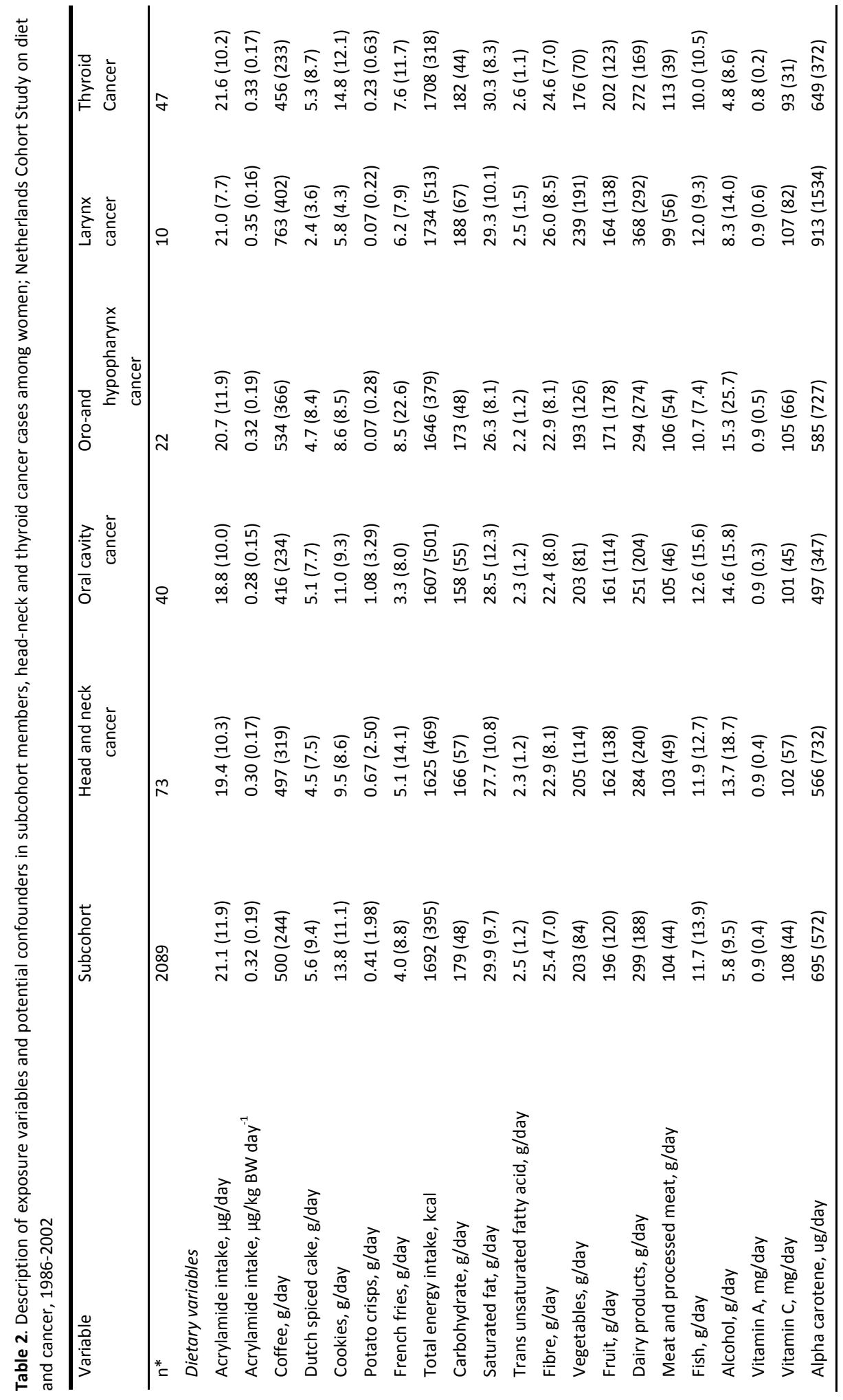




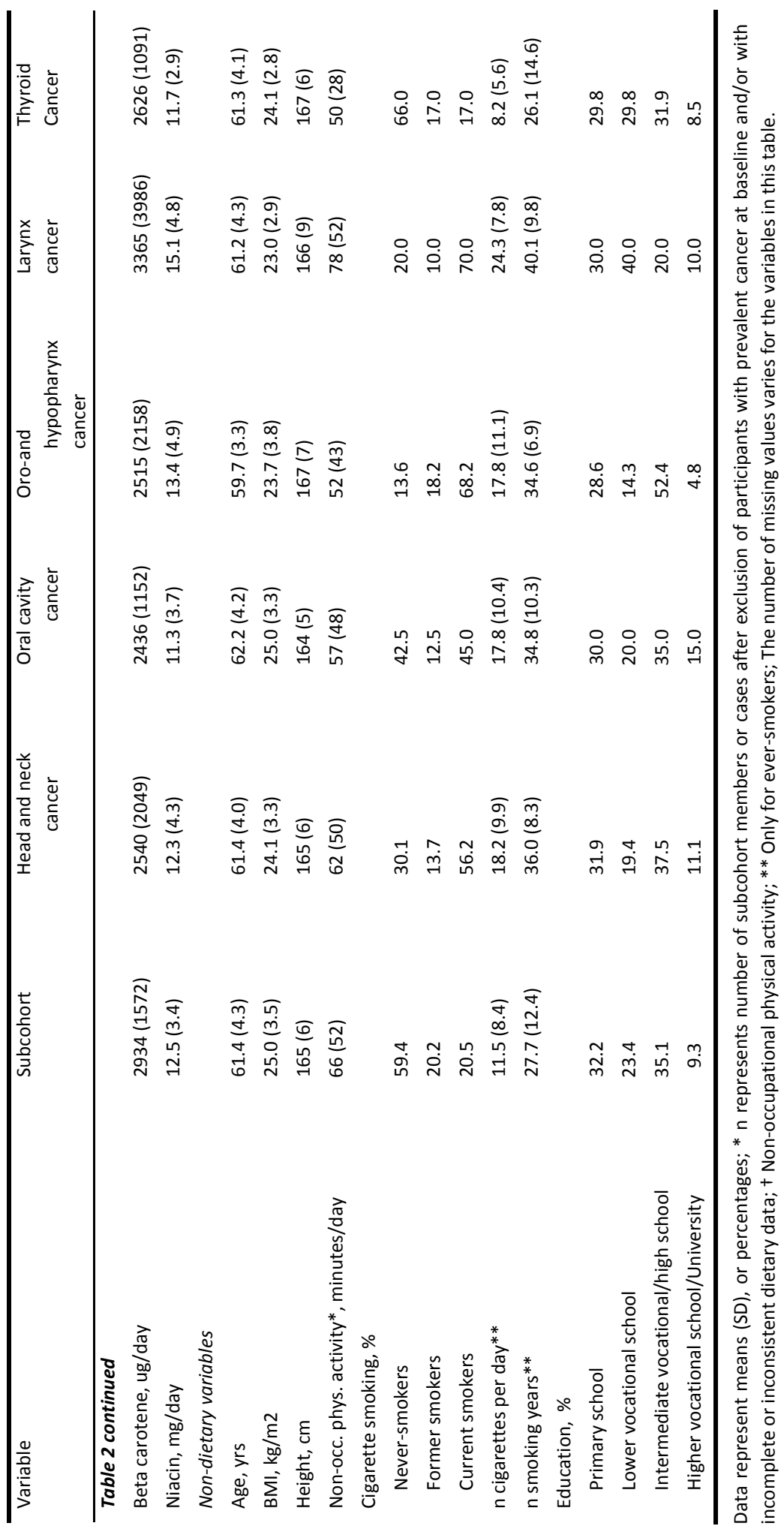




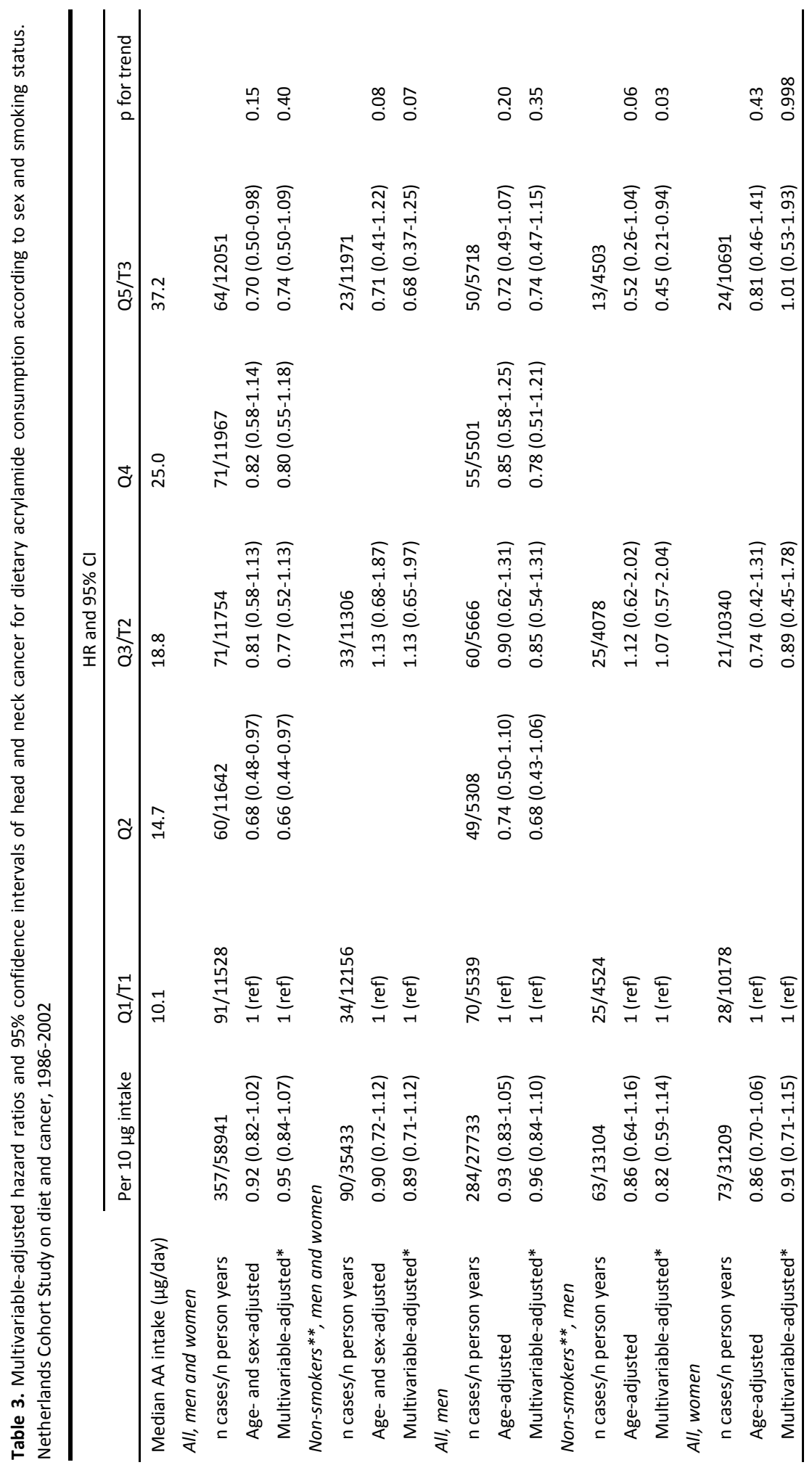


Dietary acrylamide intake and head/neck and thyroid cancer risk

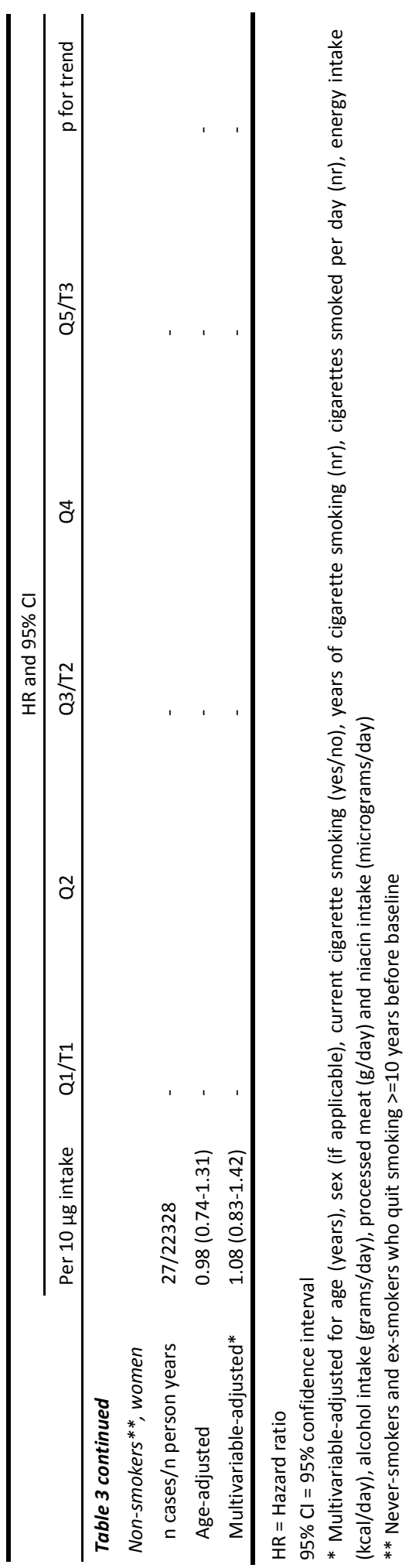




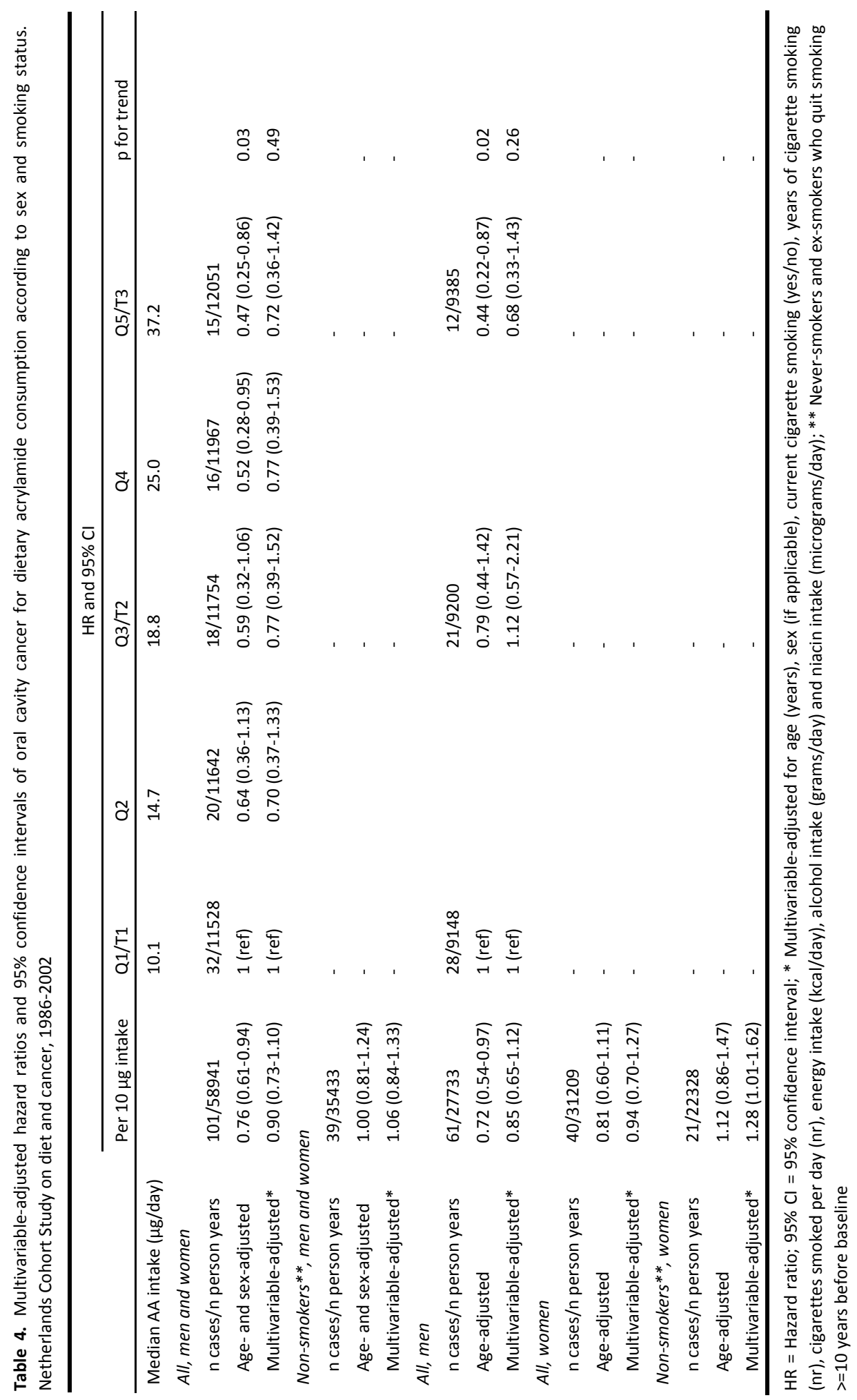


Dietary acrylamide intake and head/neck and thyroid cancer risk

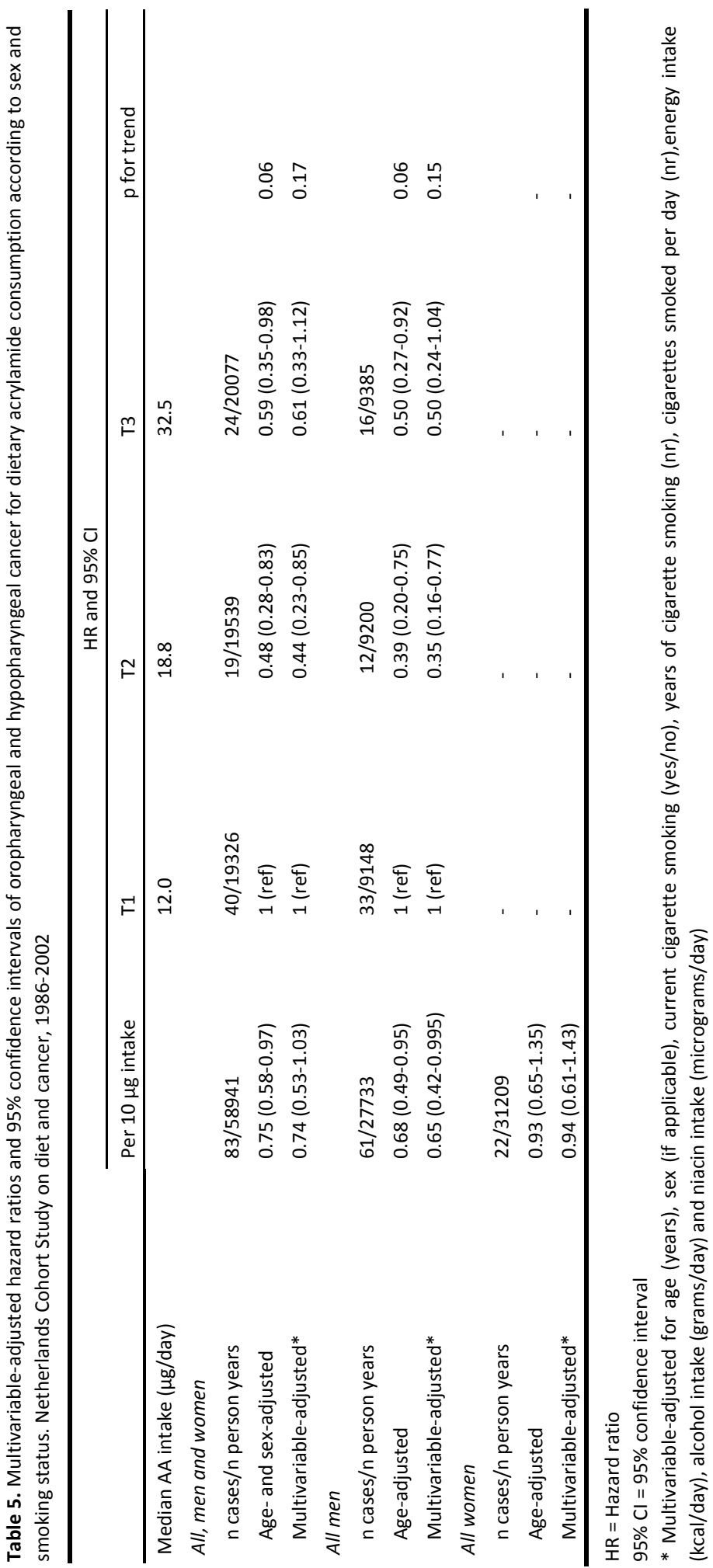




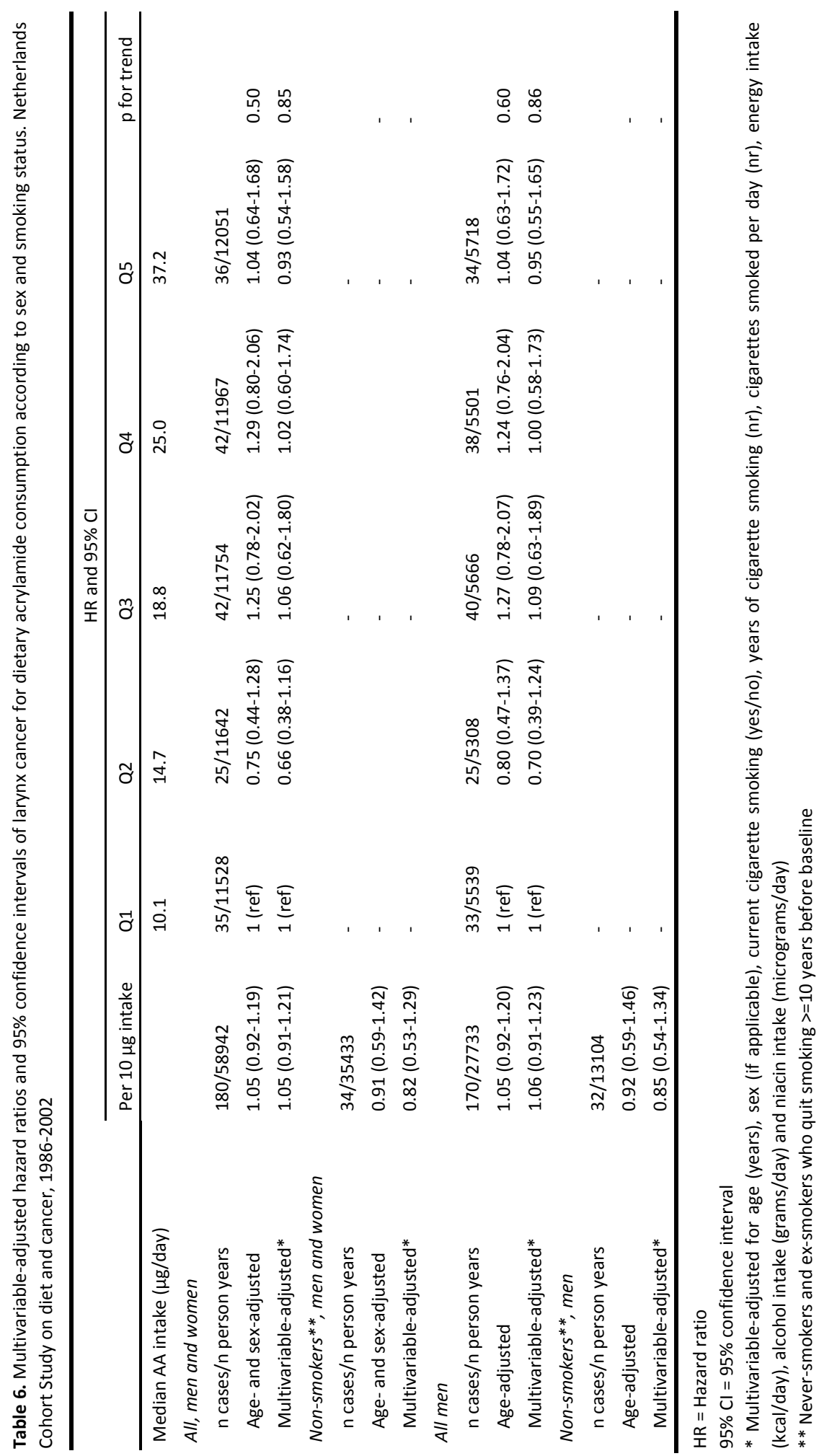




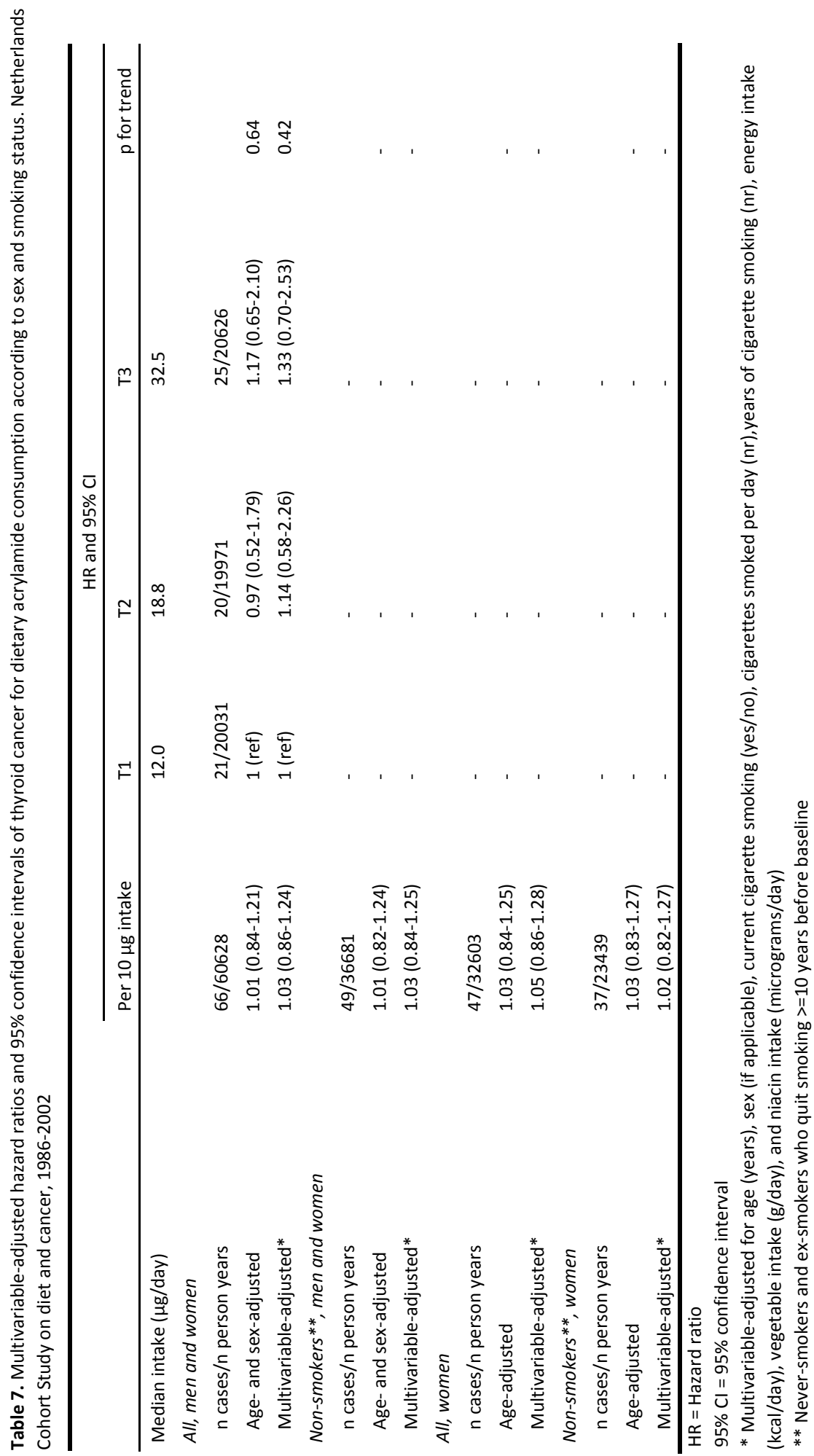


There were some indications for a decreased risk in men with increasing intake of dietary acrylamide, especially with respect to oral cavity and oro- and hypopharynx cancers. Dietary acrylamide was statistically significantly associated with an increased risk of oral cavity cancer in non-smoking women only, but numbers were small.

To our knowledge, the association between dietary acrylamide intake and the risk of head-neck cancer has been studied only in one hospital-based case-control study, wherein men and women were not analysed separately (9). In that study, no association was observed with oral cavity/pharynx cancer or with larynx cancer. The absence of a positive association is in agreement with our findings. In occupational studies, no associations were found either between acrylamide exposure and head-neck cancer, although in these studies the number of cases was small and adjustment for confounding was limited $(7,8)$. We observed a positive association between dietary acrylamide intake and the risk of oral cavity cancer in non-smoking women, but numbers were small. In earlier studies, we observed positive associations between dietary acrylamide and endometrial, ovarian and renal cell cancers $(10,11)$, an inverse association with lung cancer in women $(28)$ and we have suggested that acrylamide might be carcinogenic through a hormonal mechanism. Although hormonal factors have been associated with oral cancer in women $(29,30)$, it cannot be excluded that our finding is a chance finding.

To our knowledge, the association between dietary acrylamide and the risk of thyroid cancer in humans has not been studied before. Statistically non-significantly increased risks have been observed, however, for occupational exposure to acrylamide $(7,8)$. The numbers of cases were very small in these studies. Furthermore, the possibility to control for confounders, especially smoking, was limited in these occupational studies. Finally, in one study, the cases were detected in the low exposure group (8), whereas in the other study, the strength of the association became weaker with longer follow-up (7), making a causal relationship less likely.

In the two available chronic studies in rats, acrylamide exposure (administered in drinking water) was associated with an increased incidence of oral tissue tumours (5) and thyroid cancer $(4,5)$. The associations were only observed in the highest exposure group ( $2 \mathrm{mg} / \mathrm{kg}$ body weight/day) $(4,5)$, which is about four thousand times higher than the estimated dietary intake by participants of this cohort (23). No statistically significantly increased incidence of these cancers was observed at lower doses in these studies. The positive finding with respect to oral tissue tumours in one study was not replicated in the other study with rats (4). The positive association with thyroid cancer was observed in both studies in which rats were exposed to acrylamide $(4,5)$. This outcome has been attributed to both a mutagenic mode of action and thyroid growth stimulation (31). An important difference between rats and humans is the absence of thyroid-binding globulin in rodents (31). Besides the difference in dosage and the exposure route, this might explain the absence of an association in humans. The tumours that developed in rats were of the follicular type. When we restricted our analysis to this subgroup, no association was observed either. It seems unlikely that differences in acrylamide metabolisation explain the observed differences between rodents and humans. The metabolical pathways are quite similar, although there are differences in metabolisation rate. Of the investigated 
species, mice have the highest efficiency of acrylamide to glycidamide conversion, followed by rats and then humans $(32,33)$.

It is unlikely that the results from the NLCS have been affected by selection or information bias. Selection bias is unlikely given the high level of follow-up in terms of cases and subcohort person-years (17). FFQs have limitations, as discussed elsewhere (34), but they are the only feasible way to assess dietary intake over a long period in large-scale epidemiological studies. The NLCS FFQ has proved to be both valid (18) and reproducible (19), also with regard to nutrients that correlate with dietary acrylamide, such as carbohydrates and fibre. An important asset of the present study is that we used acrylamide concentrations from the Dutch market only and specifically sampled and analysed foods that were relevant for the NLCS population. The sampling of food items was based on a sampling plan, in which representativeness of the market, regional variations, and home cooking were taken into account. Within foods, acrylamide concentrations can vary greatly, which could lead to non-differential misclassification of acrylamide intake. For this reason, we used the mean acrylamide value of several analysed samples of each food for our acrylamide food database. In a study of 39 subjects providing a 24-h duplicate diet in 2004, we compared the 24-h dietary acrylamide intake calculated from the mean acrylamide values from our database with the chemically analysed intake from the 24-h diet and found a Spearman correlation coefficient of 0.82 (Konings et al., see Chapter 2 of this thesis). This indicates that the use of this acrylamide database does not introduce much misclassification or attenuation. Acrylamide values in our database (sampled and analysed in 2002 and 2005) may have been different from the actual values at baseline of this study in 1986. However, we assume that this misclassification is non-differential and this most likely would have biased our results toward the null value (10).

Smoking is an important source of acrylamide and is an important risk factor for head and neck cancer. Because of the small number of never-smokers, in most analyses, we combined never-smokers with former smokers that quit smoking more than ten years before baseline. We reasoned that this would enable us to reduce the misclassification of acrylamide exposure due to smoking. Although HRs among these former smokers were increased (not statistically significant) compared with never-smokers, these HRs were lower than those of current smokers and former smokers that quit smoking shortly before baseline. Occupational exposure is another source of acrylamide exposure. We were not able to restrict our analyses to cohort members that were not occupationally exposed to acrylamide. In the Netherlands, only one factory has been producing acrylamide from 1965 onwards and considering the number of people employed, it is unlikely that NLCS cohort members have been employed. Exposure to acrylamide can happen in many other occupations, e.g. biotechnology laboratories, but it was not possible to identify these exposed cohort members in the NLCS. This might have caused confounding, but only if occupational acrylamide exposure is associated with dietary acrylamide exposure, which seems improbable.

Adjustment of the dietary acrylamide HR for Dutch spiced cake had a considerable effect on the dietary acrylamide HR for some cancer types. However, Dutch spiced cake itself was (when included in a model without dietary acrylamide) not associated with any 
of the cancer endpoints. Therefore, the observed associations for dietary acrylamide are probably not confounded by other characteristics of Dutch spiced cake and coffee. The observed changes in the dietary acrylamide HRs when adjusted for Dutch spiced cake are perhaps due to instability (as shown by the substantially increased confidence intervals) of the Dutch spiced cake-adjusted HR of dietary acrylamide, caused by the high correlation between dietary acrylamide intake and Dutch spiced cake.

In conclusion, we did not observe a positive association between dietary acrylamide intake and the risk of head-neck and thyroid cancers, with a possible exception for oral cavity cancer in non-smoking women. We observed some indications for an inverse association between increasing intake of dietary acrylamide and the risk of oral cavity and oro- and hypopharynx cancers in men. However, the number of cases in specific subgroups was small and we therefore encourage other researchers to prospectively investigate the association between dietary acrylamide intake and the risk of these cancers and to perform subgroup analyses for non- and never-smokers. 


\section{References}

1. International Agency for Research on Cancer. Monographs on the evaluation of carcinogen risk to humans: some industrial chemicals. Lyon: International Agency for Research on Cancer, 1994.

2. Bull RJ, Robinson M, Laurie RD, et al. Carcinogenic effects of acrylamide in Sencar and A/J mice. Cancer Res 1984;44:107-11.

3. Bull RJ, Robinson M, Stober JA. Carcinogenic activity of acrylamide in the skin and lung of Swiss-ICR mice. Cancer Lett 1984;24:209-12.

4. Friedman MA, Dulak LH, Stedham MA. A lifetime oncogenicity study in rats with acrylamide. Fundam Appl Toxicol 1995;27:95-105.

5. Johnson KA, Gorzinski SJ, Bodner KM, et al. Chronic toxicity and oncogenicity study on acrylamide incorporated in the drinking water of Fischer 344 rats. Toxicol Appl Pharmacol 1986;85:154-68.

6. Tareke E, Rydberg P, Karlsson P, Eriksson S, Tornqvist M. Analysis of acrylamide, a carcinogen formed in heated foodstuffs. J Agric Food Chem 2002;50:4998-5006.

7. Marsh GM, Youk AO, Buchanich JM, Kant IJ, Swaen G. Mortality patterns among workers exposed to acrylamide: updated follow up. J Occup Environ Med 2007;49:82-95.

8. Swaen GM, Haidar S, Burns CJ, et al. Mortality study update of acrylamide workers. Occup Environ Med 2007;64:396-401.

9. Pelucchi C, Galeone C, Levi F, et al. Dietary acrylamide and human cancer. Int J Cancer 2006;118:467-71.

10. Hogervorst JG, Schouten LJ, Konings EJ, Goldbohm RA, van den Brandt PA. A prospective study of dietary acrylamide intake and the risk of endometrial, ovarian, and breast cancer. Cancer Epidemiol Biomarkers Prev 2007;16:2304-13.

11. Hogervorst JG, Schouten LJ, Konings EJ, Goldbohm RA, van den Brandt PA. Dietary acrylamide intake and the risk of renal cell, bladder, and prostate cancer. Am J Clin Nutr 2008;87:1428-38.

12. Olesen PT, Olsen A, Frandsen H, Frederiksen K, Overvad K, Tjonneland A. Acrylamide exposure and incidence of breast cancer among postmenopausal women in the Danish Diet, Cancer and Health Study. Int J Cancer 2008;122:2094-100.

13. Friedman M. Chemistry, biochemistry, and safety of acrylamide. A review. J Agric Food Chem 2003;51:450426.

14. Ikeda GJ, Miller E, Sapienza PP, Michel TC, Inskeep PB. Comparative tissue distribution and excretion of [114C]acrylamide in beagle dogs and miniature pigs. Food Chem Toxicol 1987;25:871-5.

15. van den Brandt PA, Goldbohm RA, van 't Veer P, Volovics A, Hermus RJ, Sturmans F. A large-scale prospective cohort study on diet and cancer in The Netherlands. J Clin Epidemiol 1990;43:285-95.

16. Prentice RL. A case-cohort design for epidemiologic cohort studies and disease prevention trials. Biometrika 1986;72:1-11.

17. Goldbohm RA, Van den Brandt PA, Dorant E. Estimation of the coverage of Dutch municipalities by cancer registries and PALGA based on hospital discharge data. Tijdschr Soc Gezondheidsz 1994;72:80-84.

18. Goldbohm RA, van den Brandt PA, Brants HA, et al. Validation of a dietary questionnaire used in a largescale prospective cohort study on diet and cancer. Eur J Clin Nutr 1994;48:253-65.

19. Goldbohm RA, van 't Veer P, van den Brandt PA, et al. Reproducibility of a food frequency questionnaire and stability of dietary habits determined from five annually repeated measurements. Eur J Clin Nutr 1995;49:420-9.

20. Van den Brandt PA, Schouten LJ, Goldbohm RA, Dorant E, Hunen PM. Development of a record linkage protocol for use in the Dutch Cancer Registry for Epidemiological Research. Int J Epidemiol 1990;19:553-8.

21. Schottenfeld D, Fraumeni Jr. JF. Cancer Epidemiology and Prevention. Third edition. Oxford: Oxford University Press, 2006.

22. Hashibe M, Brennan P, Benhamou S, et al. Alcohol drinking in never users of tobacco, cigarette smoking in never drinkers, and the risk of head and neck cancer: pooled analysis in the International Head and Neck Cancer Epidemiology Consortium. J Natl Cancer Inst 2007;99:777-89.

23. Konings EJ, Baars AJ, van Klaveren JD, et al. Acrylamide exposure from foods of the Dutch population and an assessment of the consequent risks. Food Chem Toxicol 2003;41:1569-79.

24. Schettgen T, Rossbach B, Kutting B, Letzel S, Drexler H, Angerer J. Determination of haemoglobin adducts of acrylamide and glycidamide in smoking and non-smoking persons of the general population. Int J Hyg Environ Health 2004;207:531-9.

25. Schoenfeld D. Partial residuals for the proportional hazards regression model. Biometrika 1982;69:239-241.

26. Cox DR. Regression models and life-tables (with discussion). J R Statist Soc B 1972;34:187-220. 


\section{Chapter 8}

27. Lin DY, Wei LJ. The robust inference for the Cox Proportional Hazards Model. J Am Stat Assoc 1989;84:10741078.

28. Hogervorst JGF, Schouten LJ, Konings EJ, Goldbohm RA, van den Brandt PA. Lung cancer risk in relation to dietary acrylamide intake. Journal of the National Cancer Institute 2009, in press.

29. Bosetti C, Negri E, Franceschi S, et al. Risk factors for oral and pharyngeal cancer in women: a study from Italy and Switzerland. Br J Cancer 2000;82:204-7.

30. Suba Z. Gender-related hormonal risk factors for oral cancer. Pathol Oncol Res 2007;13:195-202.

31. Dourson M, Hertzberg R, Allen B, et al. Evidence-based dose-response assessment for thyroid tumorigenesis from acrylamide. Regul Toxicol Pharmacol 2008;52:264-89.

32. Konings EJ, Hogervorst JGF, Schouten L, van den Brandt PA. Assessing exposure levels of acrylamide. In: Skog K, Alexander J, eds. Acrylamide and other hazardous compounds in heat-treated foods. Cambridge: Woodhead Publishing Limited, 2006:214-225. 


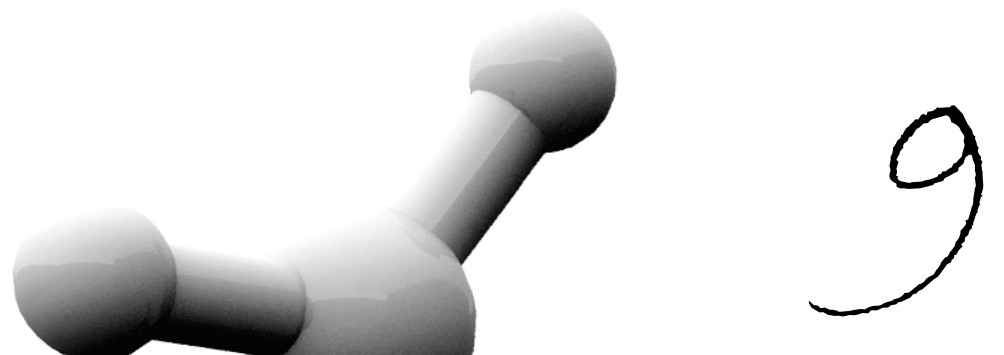

The carcinogenicity of dietary acrylamide intake: a side by side discussion of epidemiological and toxicological research

Janneke G.F. Hogervorst Leo J. Schouten

Erik J.M. Konings

R. Alexandra Goldbohm

Piet A. van den Brandt 


\section{Background}

Seven years after acrylamide (a known rodent carcinogen and probable human carcinogen) was discovered in everyday foods, like French fries, crisps, and coffee, the discussion about what dietary acrylamide intake means in terms of human cancer risk is still vivid. Initially, risk assessments from, among others, the US Environmental Protection Agency and the World Health Organization based on linear extrapolations from the rodent results, indicated that the levels of dietary acrylamide intake in the Western world would lead to unacceptable numbers of excess human cancer cases (1-3). This raised a lot of concern in the scientific community, and rendered considerable media attention.

Since 2002, several epidemiological studies have been undertaken that can be compared with the data that are available from chronic rodent acrylamide carcinogenicity bioassays. There have now been publications from five case-control and seven prospective cohort studies that investigated the link between dietary acrylamide intake and the risk of several types of human cancer, of which some looked into multiple cancer sites per study. The initial epidemiological studies (mostly case-control studies) did not observe positive associations between dietary acrylamide intake and risk of several cancer types. This led to a general feeling that the human risks were probably too low to be detected in epidemiological studies, which would indeed be the case if the excess lifetime cancer risk based on the high dose animal data applies to low doses in humans. However, in 2007 and 2008 two prospective cohort studies observed increased risks for some cancer types in relation to dietary acrylamide intake (4-6), and in one of these studies for multiple cancer sites.

In May 2008, the European Food Safety Authority (EFSA) convened a scientific colloquium to review the new evidence (both toxicological and epidemiological) on acrylamide carcinogenicity. Although human data are often considered the most important input data for human risk assessment and there were now epidemiological data suggesting that dietary acrylamide intake may constitute an important carcinogenic risk in humans, the outcome of a voting among the scientific audience (mainly consisting of toxicologists) of the EFSA colloquium was that the level of concern on acrylamide carcinogenicity was not increased among the majority of voters (7). We therefore want to draw attention to the apparent discrepancy between the worlds of toxicologists and epidemiologists involved in acrylamide cancer research and to compare the data from both research fields in this review.

We will discuss the body of evidence from the epidemiological and rodent studies on dietary acrylamide carcinogenicity, including strengths and weaknesses and variability, how both types of evidence relate and possible reasons for discrepancies, a possible hormonal pathway of acrylamide carcinogenicity, recommendations for future epidemiological acrylamide research, and implications for public health. 


\section{Review of epidemiological studies}

\section{Methods}

We focus on human cancer risks associated with dietary acrylamide intake. Studies on occupational acrylamide exposure are not included, because exposure pathways are different in those studies, they lack thorough adjustment for confounders, and contain virtually only men. Furthermore, the review does not include studies on single acrylamidecontaining foods, such as fried potatoes, because we consider those exposures not representative of total acrylamide intake, since many different foods contribute to dietary acrylamide exposure. It is then impossible to indicate whether acrylamide or another component of the food under study is responsible for any observed associations. The search of articles was conducted through Pubmed, using the search terms acrylamide and cancer combined.

We have not pooled the results of individual studies per cancer site, because there are only few results available per cancer site (six for breast cancer, which is the highest number), and because there is considerable obvious heterogeneity among those few studies. The earlier studies were generally case-control studies, whereas the latter ones were mostly prospective cohort studies. Furthermore, later studies had more extensive data on acrylamide in foods and data that were specific for the population under study, which implies that the exposure assessment of the later studies was of better quality. For breast cancer, some studies looked at pre- or postmenopausal women specifically, separately or jointly, and some studies investigated estrogen and/or progesterone receptor-defined subgroups. In conclusion, the studies are by nature too heterogeneous to pool.

\section{Characteristics of the epidemiological studies}

In Table 1, the studies are listed with their main characteristics in view of dietary acrylamide intake and cancer risk. Only two studies so far have used acrylamide to haemoglobin adducts as the exposure measure $(5,8)$, whereas the others all used food frequency questionnaires (FFQs). Two studies have been able to use more than one acrylamide intake measurement over time $(9,10)$. Other important aspects that differ between the studies are the number of items in the FFQs, how the acrylamide intake was assessed (based on reported frequencies of foods consumption, or on both reported frequency and reported portion sizes), the origin of the data on acrylamide concentrations in individual foods that were used in the intake assessment, and the subgroups that were investigated (e.g. men/women, smokers/non-smokers).

For analysis of the acrylamide-cancer link, it is important to thoroughly control for smoking, because cigarette smoke contains a lot of acrylamide. Smokers have on average four times higher levels of acrylamide to haemoglobin adducts than non-smokers (11), and thus acrylamide exposure from smoking might cloud the assessment of the risk associated with dietary acrylamide exposure. Furthermore, cigarette smoke is a major risk factor for many types of cancer and thus has a very strong confounding potential. Besides acrylamide, cigarette smoke contains a plethora of other carcinogenic compounds. The most thorough way to control for smoking is to restrict analyses to non-smokers. 


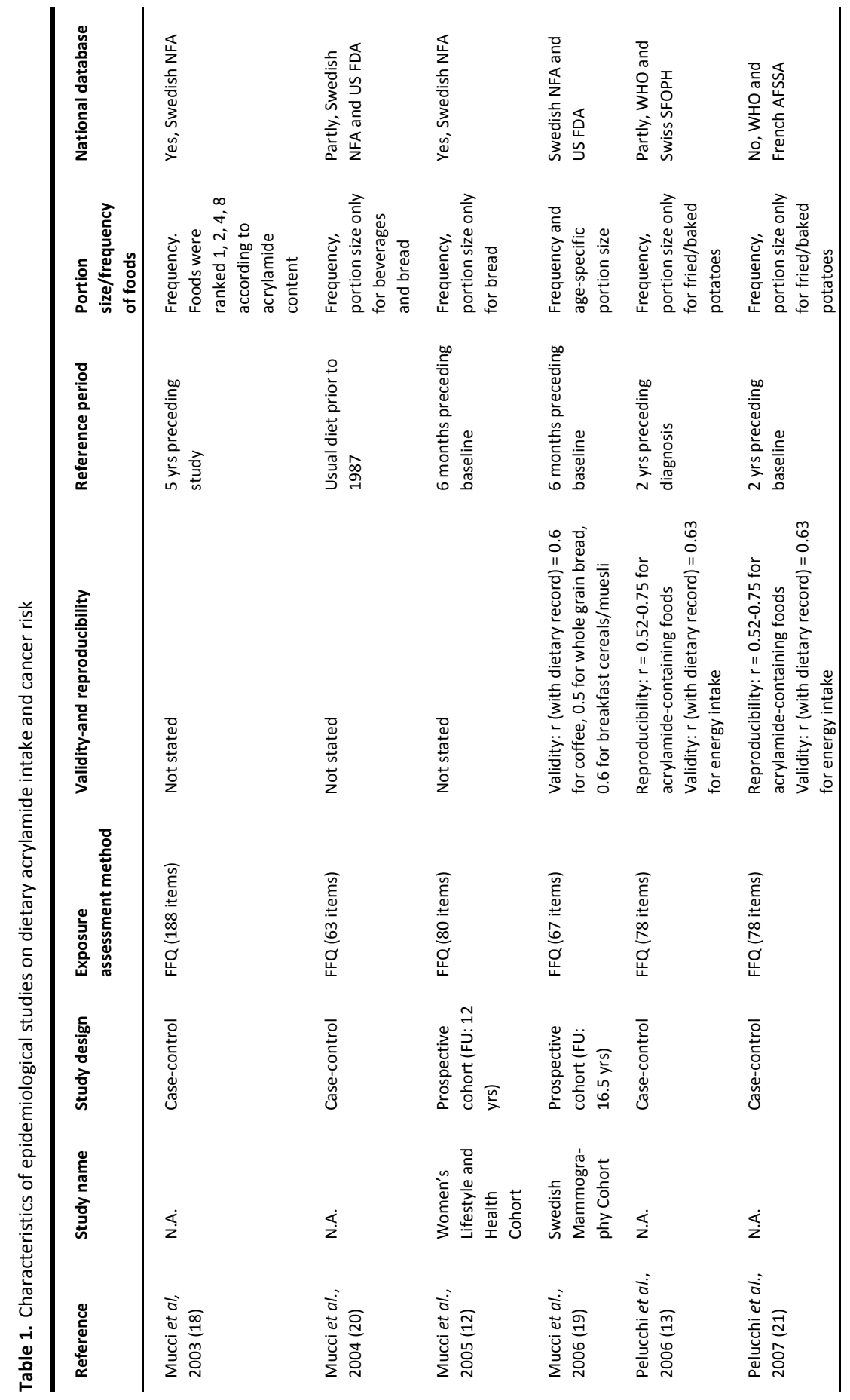




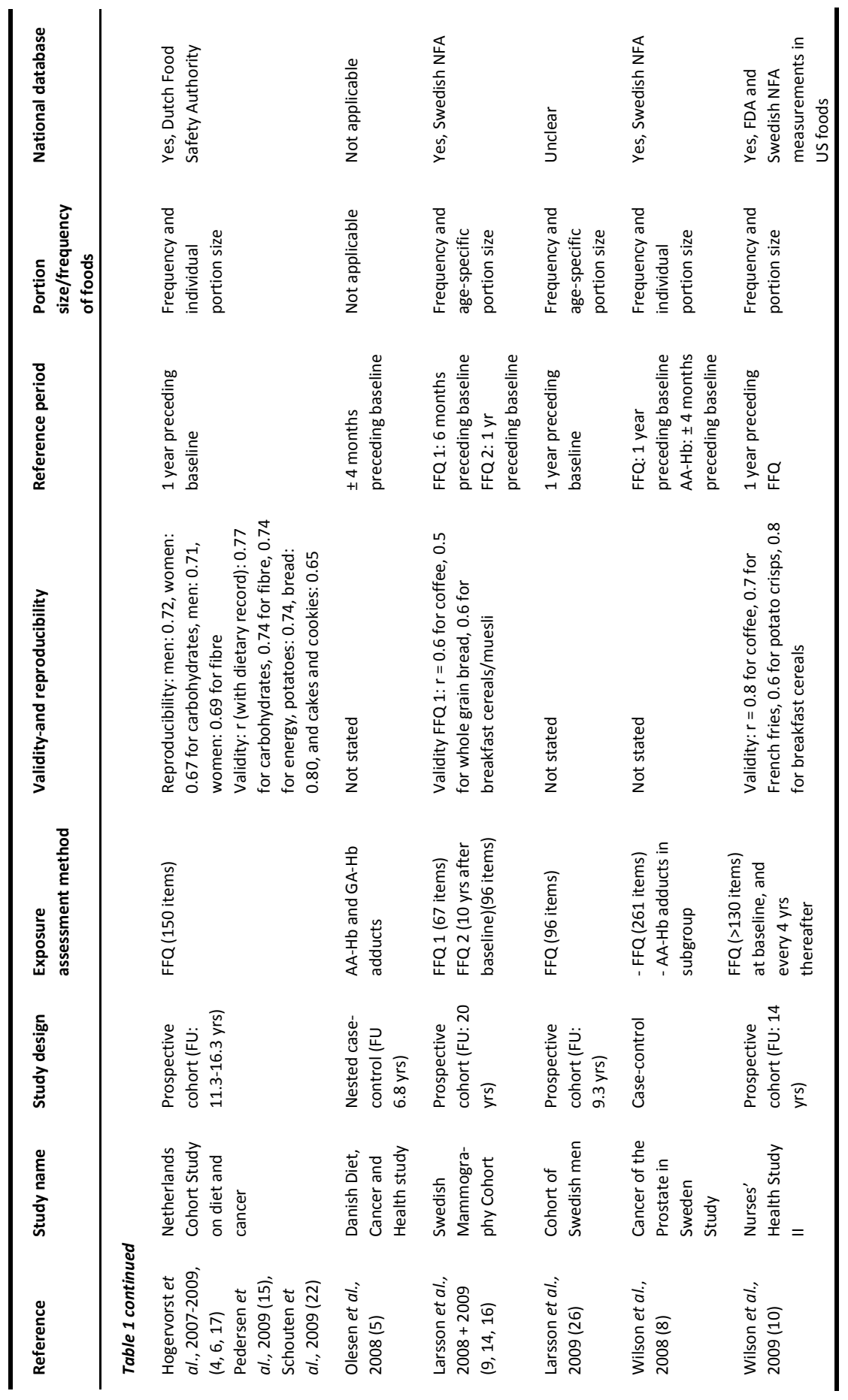




\section{Results of the epidemiological studies}

In Tables 2 through 6, the results of the epidemiological studies on dietary acrylamide intake and cancer risk are presented, using one Table per organ system.

The cancer sites that have been studied in relation to acrylamide intake are female breast $(4,5,12-15)$, endometrium $(4,9)$, ovary $(4,13,16)$, prostate $(6,8,13)$, esophagus $(13,17)$, stomach $(17)$, colorectum $(13,17-19)$, pancreas $(17)$, urinary bladder $(6,18)$, kidney $(6,18,20,21)$, oral cavity and/or hypo- or oropharynx $(13,22)$, larynx $(13,22)$, lung (23), brain (24), and thyroid (22), and one study looked at total cancer risk (Hogervorst, unpublished findings).

\section{Reproductive organs}

Table 2 contains the results of the studies on reproductive organs that have been published up to now. At the EFSA's 11th Scientific Colloquium "Acrylamide carcinogenicity New evidence in relation to dietary exposure" in May 2008, it was announced that the United States Nurses' Health Study corroborates the positive associations observed in the Netherlands Cohort Study for endometrial and ovarian cancer (7), but the results have not yet been published, and thus this study with its details could not yet be included in Tables 1 and 2 .

None of the studies observed an increased risk of total pre- or postmenopausal breast cancer in association with acrylamide intake $(4,5,10,12-14)$. However, for sex hormone receptor-positive postmenopausal breast cancer, both the Netherlands Cohort Study and the Danish Diet, Cancer and Health Study showed some indications for an increased risk $(5,15)$. The Dutch study used an FFQ and observed statistically non-significantly increased risks for estrogen and/or progesterone receptor-positive breast cancer only in neversmokers (15), whereas the Danish study used acrylamide to haemoglobin adducts as the exposure marker and observed increased risks of estrogen receptor-positive breast cancer more strongly in smokers, although the effect modification by smoking was not statistically significant (5). In the Dutch study, the association was statistically significant after adjustment for cookies, which were themselves inversely associated with the risk. The problem connected to using an acrylamide biomarker is that it is not source-specific: it reflects both dietary exposure and exposure from other sources, such as smoking. Thus, results from analyses in a smoking subgroup may suffer from residual confounding by smoking or environmental tobacco smoke exposure. Adjusting the acrylamide to haemoglobin adduct level for smoking variables may lead to collinearity, because smoking has a strong positive effect on acrylamide haemoglobin adduct levels. For these reasons, analyses in never- smokers are preferable when using acrylamide biomarkers. In the Swedish Mammography Cohort, no association with estrogen and/or progesterone receptorpositive breast cancer risk was found (14). Compared with the Dutch and Danish cohort studies, the Swedish study had a considerably smaller range in the acrylamide intake, which hampers observing an association. Furthermore, no individual data on portion size of acrylamide-containing foods were obtained, but age-specific servings were used, which may have resulted in misclassification and thus in attenuation of the estimated risk estimates. The authors adjusted the relative risk of acrylamide for coffee intake, and they did 
Side by side discussion of animal and epidemiological acrylamide research

\begin{tabular}{|c|c|c|c|c|c|}
\hline 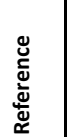 & 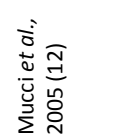 & 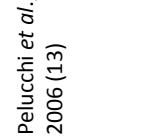 & 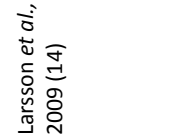 & 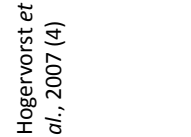 & 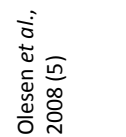 \\
\hline 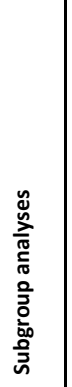 & 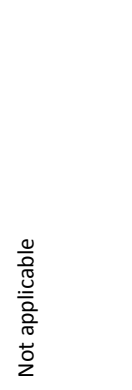 & 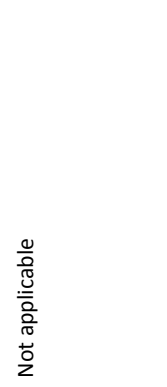 & 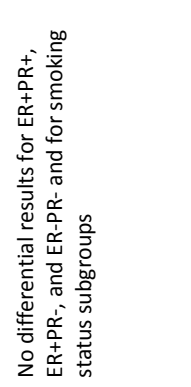 & 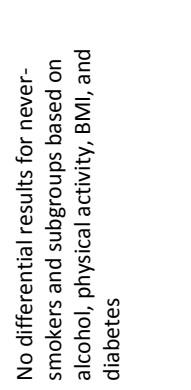 & 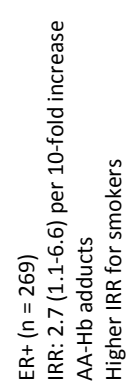 \\
\hline 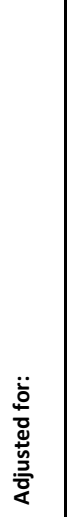 & 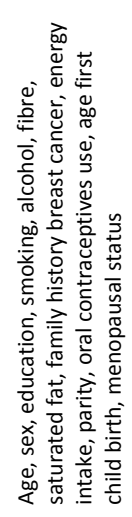 & 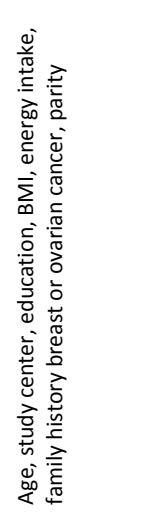 & 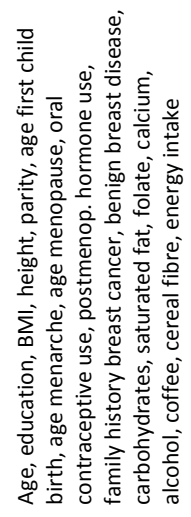 & 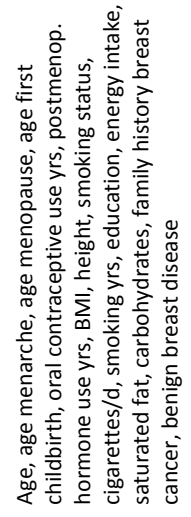 & 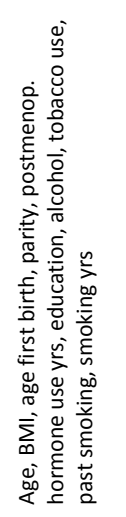 \\
\hline 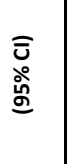 & 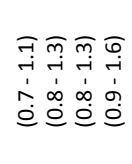 & 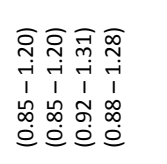 & 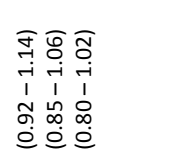 & 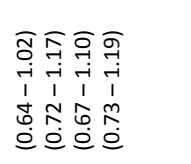 & 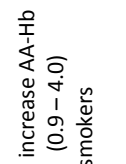 \\
\hline 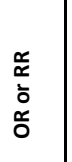 & 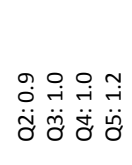 & 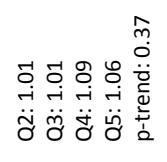 & 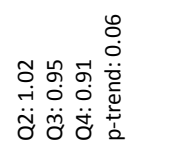 & 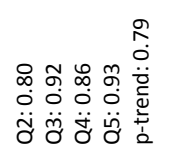 & 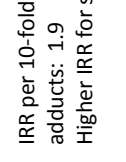 \\
\hline 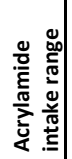 & 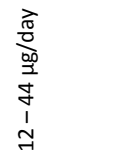 & 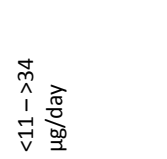 & 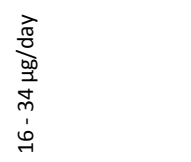 & 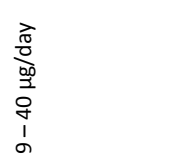 & 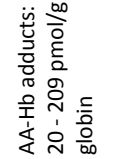 \\
\hline 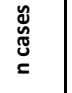 & $\widehat{\emptyset}$ & ষ্ণী & న్ న్ & 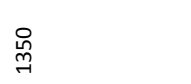 & $\underset{m}{+}$ \\
\hline 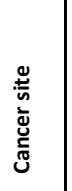 & 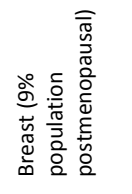 & 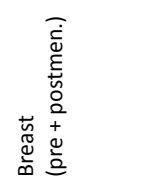 & 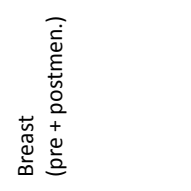 & 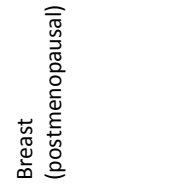 & 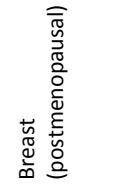 \\
\hline
\end{tabular}




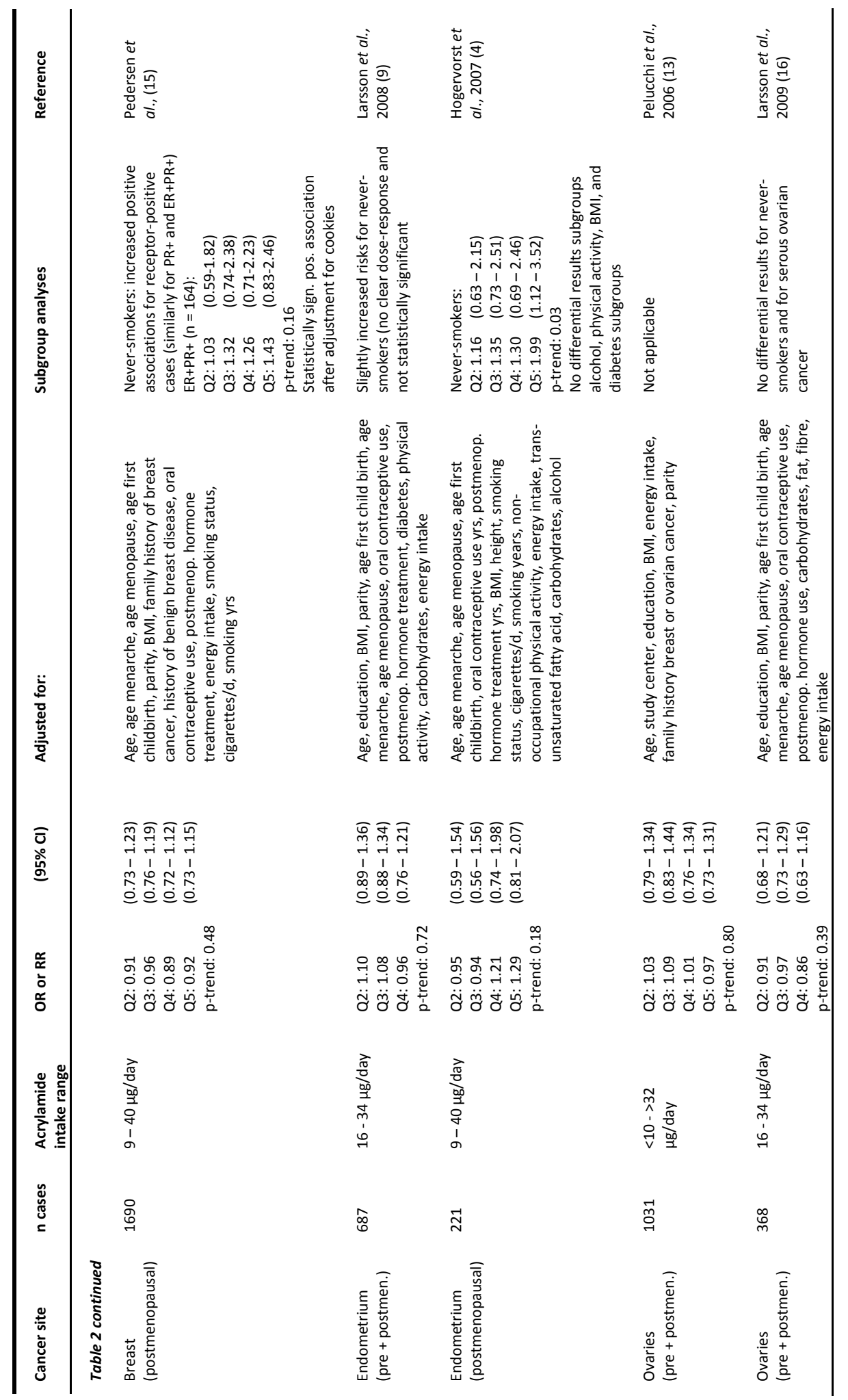


Side by side discussion of animal and epidemiological acrylamide research

\begin{tabular}{|c|c|c|c|c|c|}
\hline 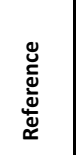 & & 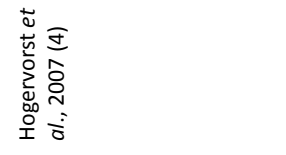 & 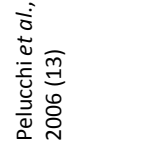 & 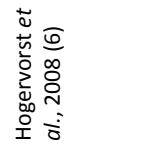 & 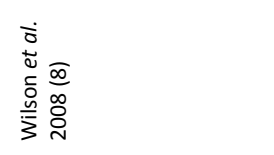 \\
\hline 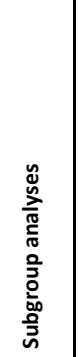 & & 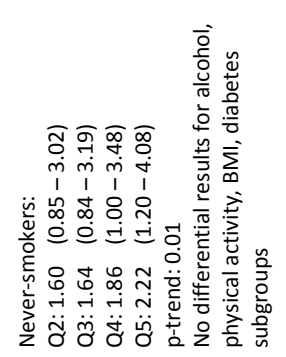 & 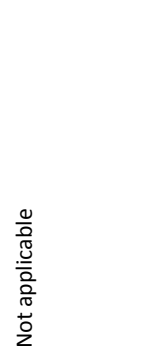 & 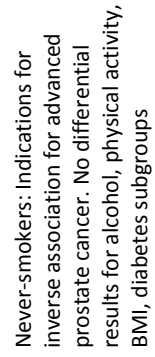 & 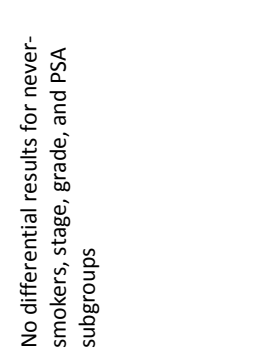 \\
\hline 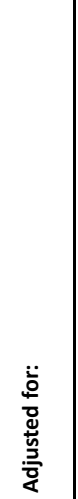 & & 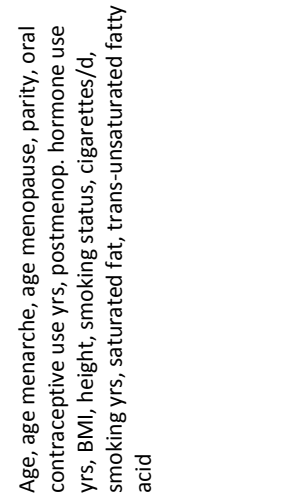 & 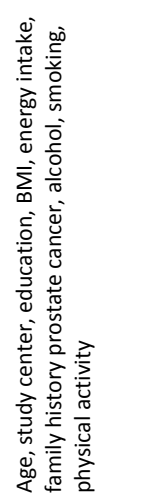 & 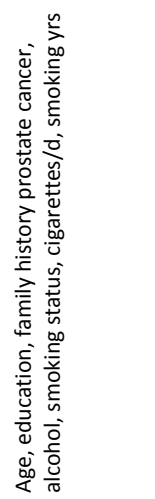 & 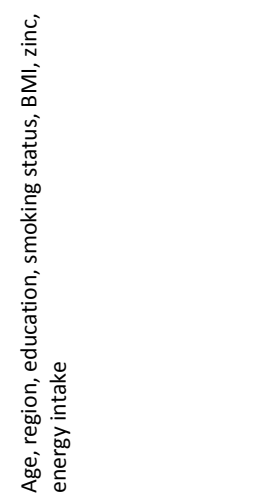 \\
\hline 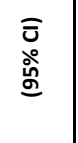 & & 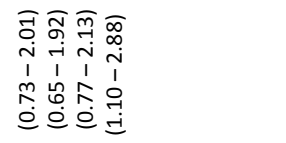 & 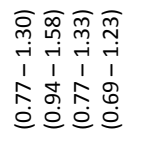 & 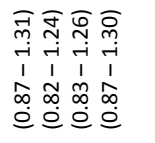 & 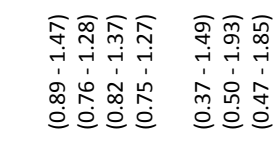 \\
\hline 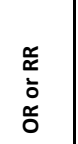 & & 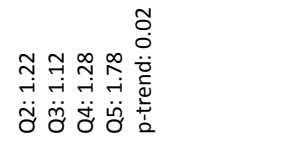 & 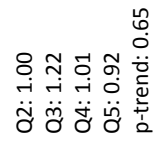 & 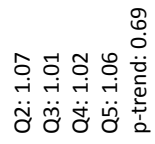 & 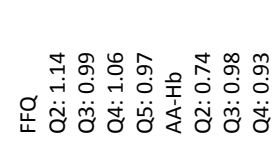 \\
\hline 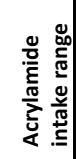 & & $\begin{array}{l}\frac{\sqrt{\pi}}{0} \\
\frac{00}{000} \\
0 \\
0 \\
1 \\
0\end{array}$ & 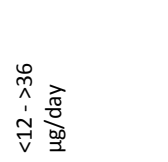 & 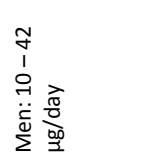 & 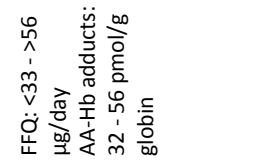 \\
\hline $\begin{array}{l}\stackrel{\mathscr{ٌ}}{~} \\
\stackrel{\Xi}{=}\end{array}$ & & 号 & $\underset{\text { స్ }}{\stackrel{+}{2}}$ & 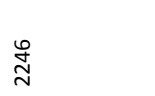 & 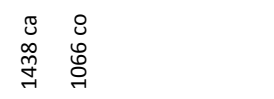 \\
\hline 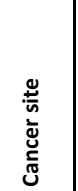 & 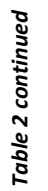 & 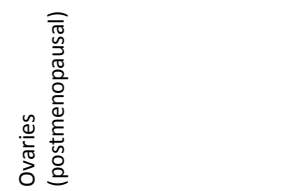 & 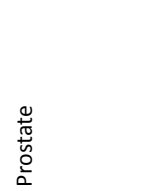 & 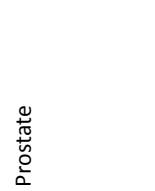 & 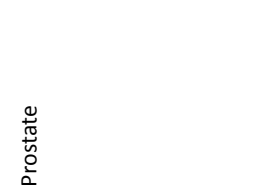 \\
\hline
\end{tabular}


not show results unadjusted for coffee. This seems strange, because coffee was by far the most important acrylamide source in their population, and adjustment might then lead to underestimation of the acrylamide effect. Although they had a repeated acrylamide intake measurement for a part of the study population, which improves the assessment of longterm intake of foods, this probably does not make up for potential imprecision due to not taking into account individually reported portion size or the small range in exposure levels. In the Nurses' Health Study II, also no association between acrylamide intake and receptor-positive premenopausal breast cancer was observed (10). Unfortunately, in the latter two studies, no stratification on both receptor status and smoking status was done.

A positive association for endometrial cancer was observed in two prospective cohort studies; the Netherlands Cohort Study on diet and cancer and the Nurses' Health Study (4, 7). No association was observed in the Swedish Mammography Cohort (9). In the Dutch study, a clear association was only found in never-smoking women (4). Interestingly, also in the Swedish study the risk estimates for never-smokers were somewhat higher (although not statistically significantly higher and with no clear dose-response relationship) than for smokers.

For ovarian cancer, a positive association was observed in the Netherlands Cohort Study and the Nurses' Health Study I $(4,7)$, but not in an Italian-Swiss case-control study (13) and the Swedish Mammography Cohort (16). In the case-control study, no separate analyses were done for pre- and postmenopausal women, and the results are therefore not directly comparable to those of the other cohort studies that did observe an association, because there are indications that the etiology of pre- and postmenopausal ovarian cancer differs (25). Further, no subgroup analyses were done based on smoking status, which would be informative, because in the Dutch study a stronger increased risk was observed in never-smokers. Both the case-control study and the Swedish cohort study did not use individually reported portion sizes in their acrylamide intake calculation, and had a lower acrylamide intake range than the Netherlands Cohort Study, especially the cohort study, which could have led to a type-II error.

Prostate cancer was studied in an Italian-Swiss case-control study, a Swedish casecontrol study, and in the Netherlands Cohort Study $(6,8,13)$. For advanced prostate cancer, the Netherlands Cohort Study showed indications for an inverse association in never-smokers (6). The $p$ value for linear trend was not significant, but a clear inverse dose-response relationship was shown. The Italian-Swiss case-control study did not show an association, but unfortunately, the authors did not specifically study never-smokers (13). The Swedish case-control study also did not show an association with prostate cancer risk (8). However, in this study it was shown that the correlation between acrylamide intake based on an FFQ and on an acrylamide biomarker (AA-Hb adducts) differed considerably between controls (Pearson $r=0.35$ ) and cases (Pearson $r=0.15$ ). This discrepancy indicates that there might have been bias in the study and hampers the interpretation of the association between acrylamide intake and prostate cancer risk in this study.

\section{Digestive system}

In Table 3, the results of the studies on cancer of the digestive system are shown. 
No overall association was observed for oesophageal cancer in a case-control and a prospective cohort study $(13,17)$, although the cohort study observed increased acrylamide-associated, especially adenocarcinoma, risks for obese persons.

Stomach cancer was studied only once, and no association with acrylamide intake was observed (17).

One study (a case-control study) (18) of the five studies (two case-control, three cohort studies) that investigated colorectal cancer found an inverse association, whereas the other four studies did not observe an association $(13,17,19)(26)$. The authors of the first study indicated that residual confounding (e.g. by dietary fibre or crisp bread) may be the explanation for this finding. The studies that stratified on colon and rectal cancer did not observe any associations for these specific sites.

Pancreatic cancer was studied only once, and the prospective cohort study did not observe an overall association with acrylamide intake (17), although increased risks were observed for obese persons, especially for microscopically verified pancreatic cancer.

\section{Urinary system}

Table 4 contains the results of the studies on cancer of the urinary system. Bladder cancer in women specifically was studied only in the Netherlands Cohort Study (6) and thus the inverse (non-statistically significant) association remains to be corroborated or refuted, but interestingly, the only other (case-control) study on bladder cancer in men and women combined showed a tendency for an inverse association in the non-smoking subgroup, which probably included relatively many women (18).

Four studies investigated the relationship between acrylamide intake and kidney or renal cell cancer risk. The only prospective study observed a positive association (6), specifically in smokers. The only other study that stratified on smoking status also observed somewhat increased acrylamide-associated risks in smokers, although not statistically significant (18).

\section{Respiratory system and head/neck}

Table 5 shows the results of the studies on cancer of the head/neck and the respiratory system. For head/neck cancer overall, the only (prospective) study observed no association with dietary acrylamide intake for men and women combined, but some indications for an inverse association for men, especially non-smokers (22). One case-control study investigated oral cavity and pharyngeal cancer combined (13), whereas a prospective study looked at these cancer types separately (22). In the case-control study, no association was observed, whereas in the cohort study a statistically significant positive association was observed in non-smoking women for oral cavity cancer, however based on only 21 cases. For oro- and hypopharyngeal cancer, the same study observed some indications for an inverse association in men, but with no clear dose-response relationship. The two studies that looked at laryngeal cancer did not show evidence for a relationship with acrylamide intake $(13,22)$.

Lung cancer was studied only in the Netherlands Cohort Study (23). For men, there was no association, but for women there was a statistically significant inverse association with 


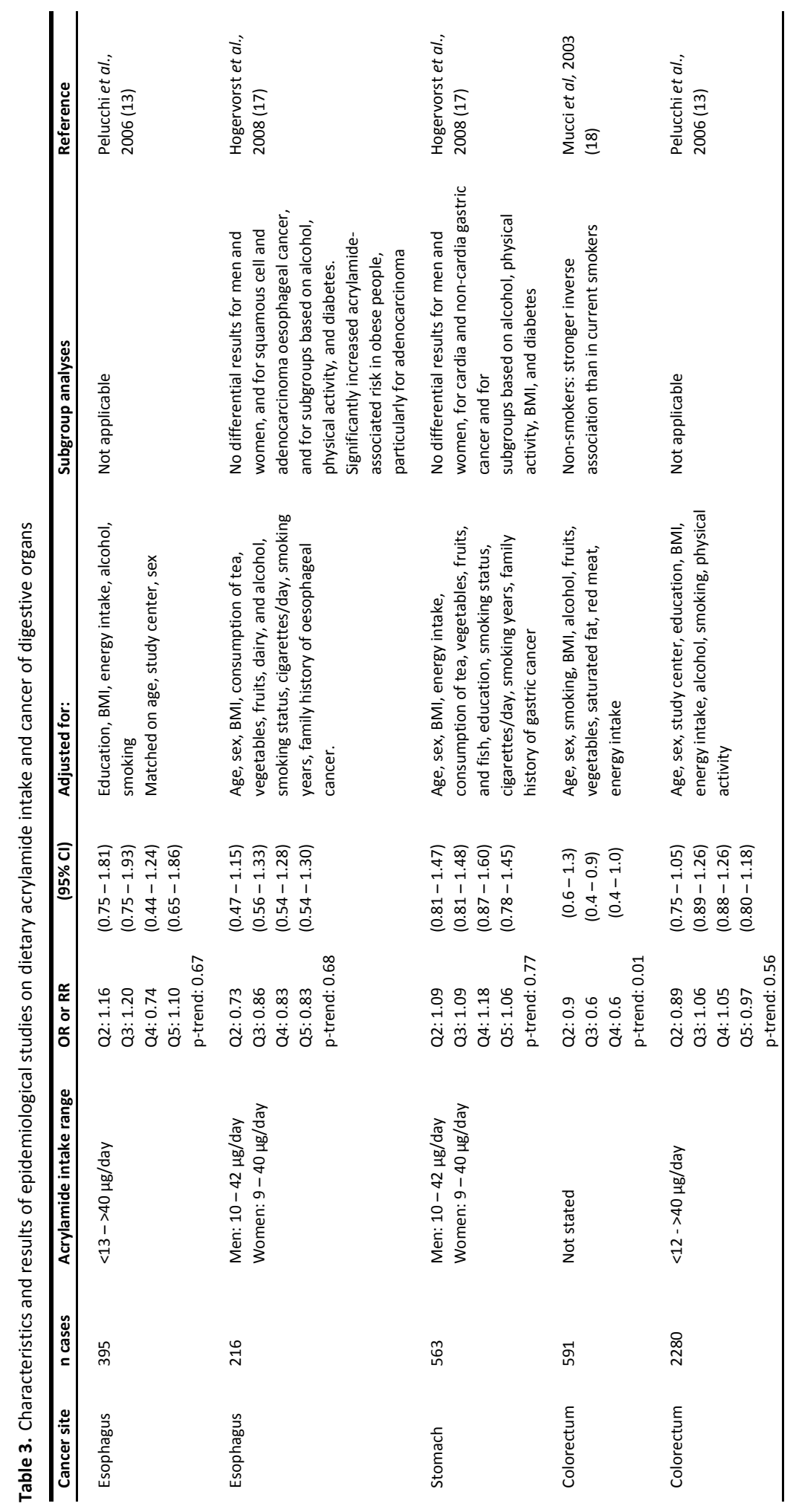




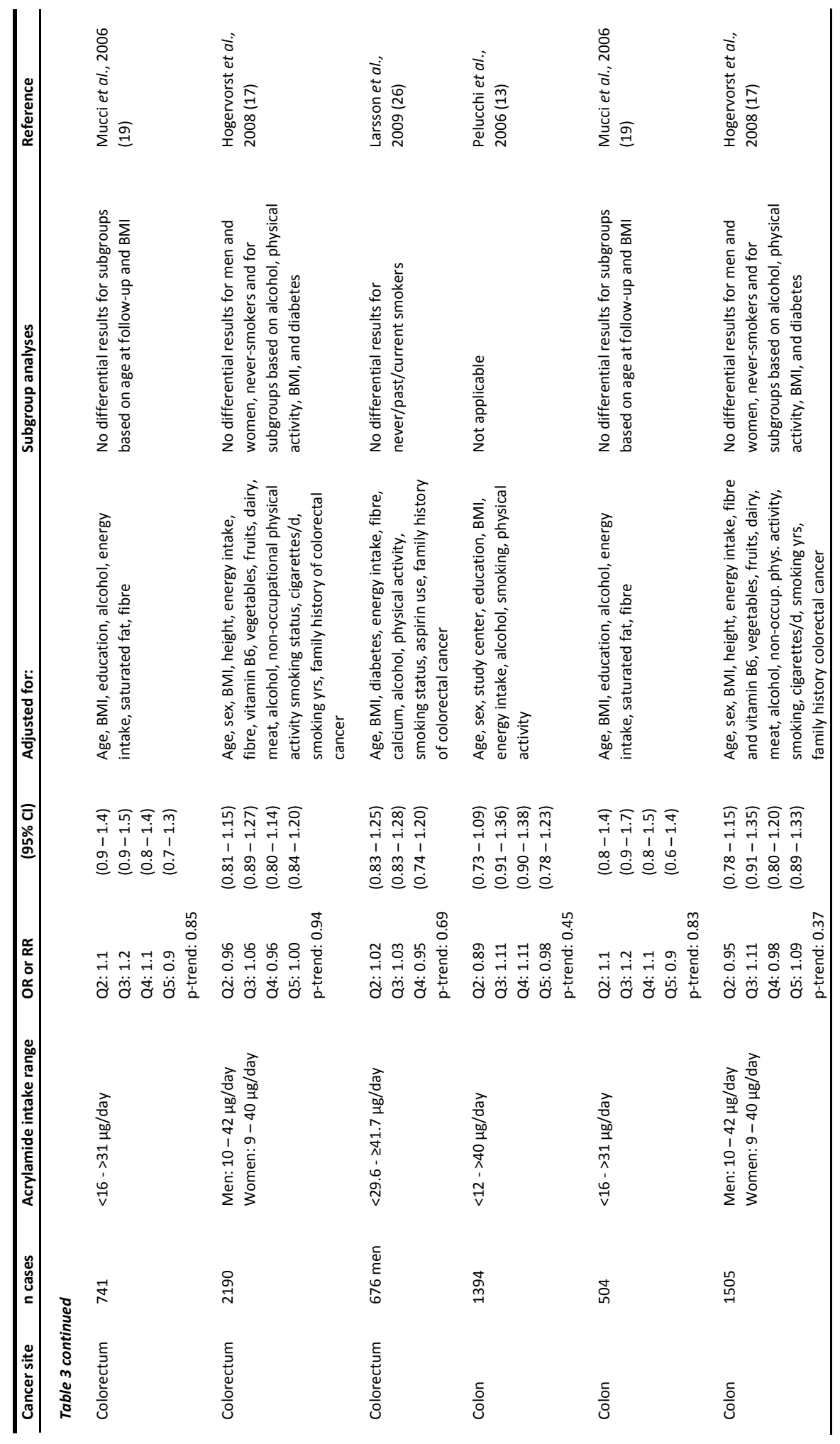




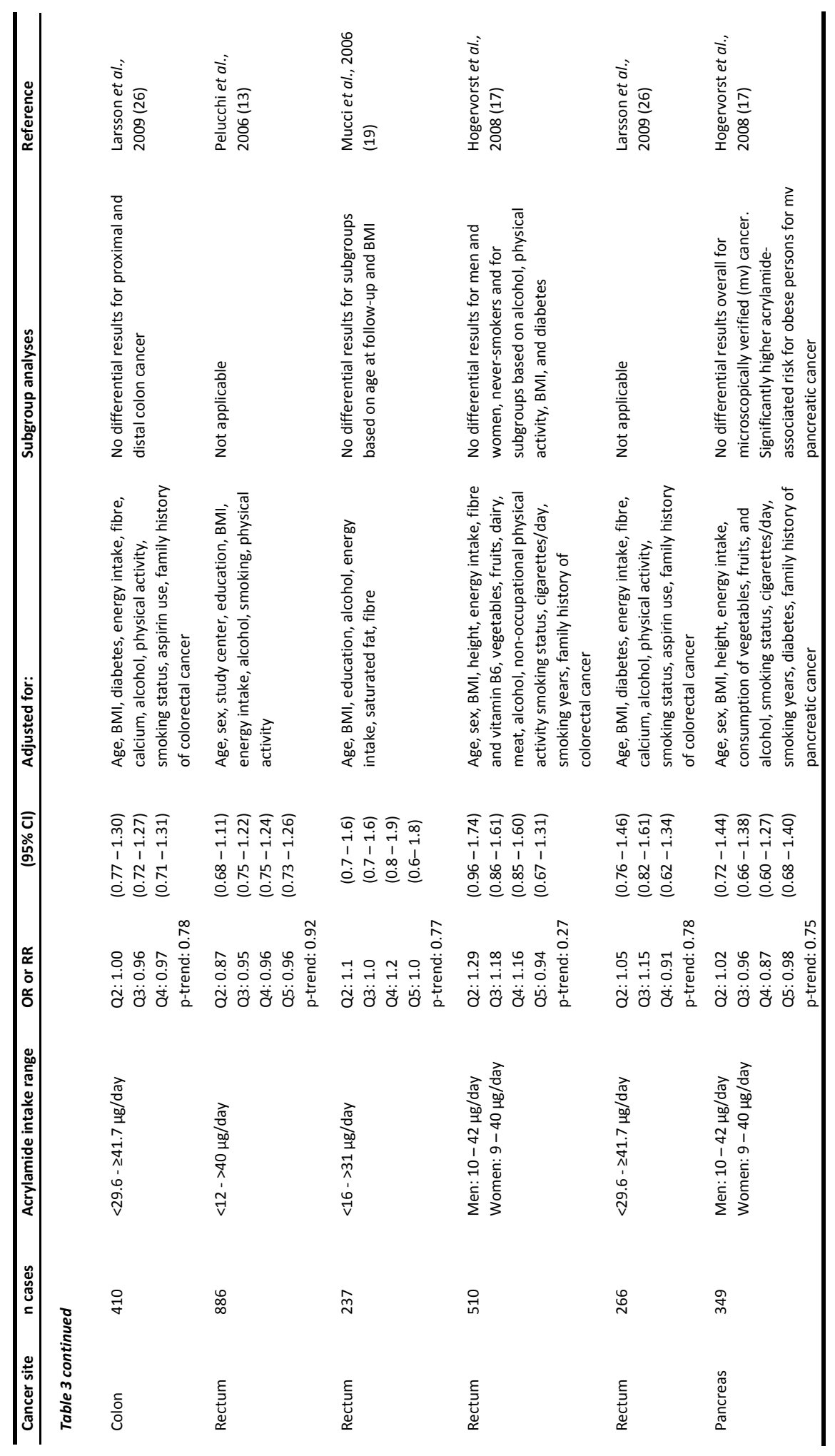


Side by side discussion of animal and epidemiological acrylamide research

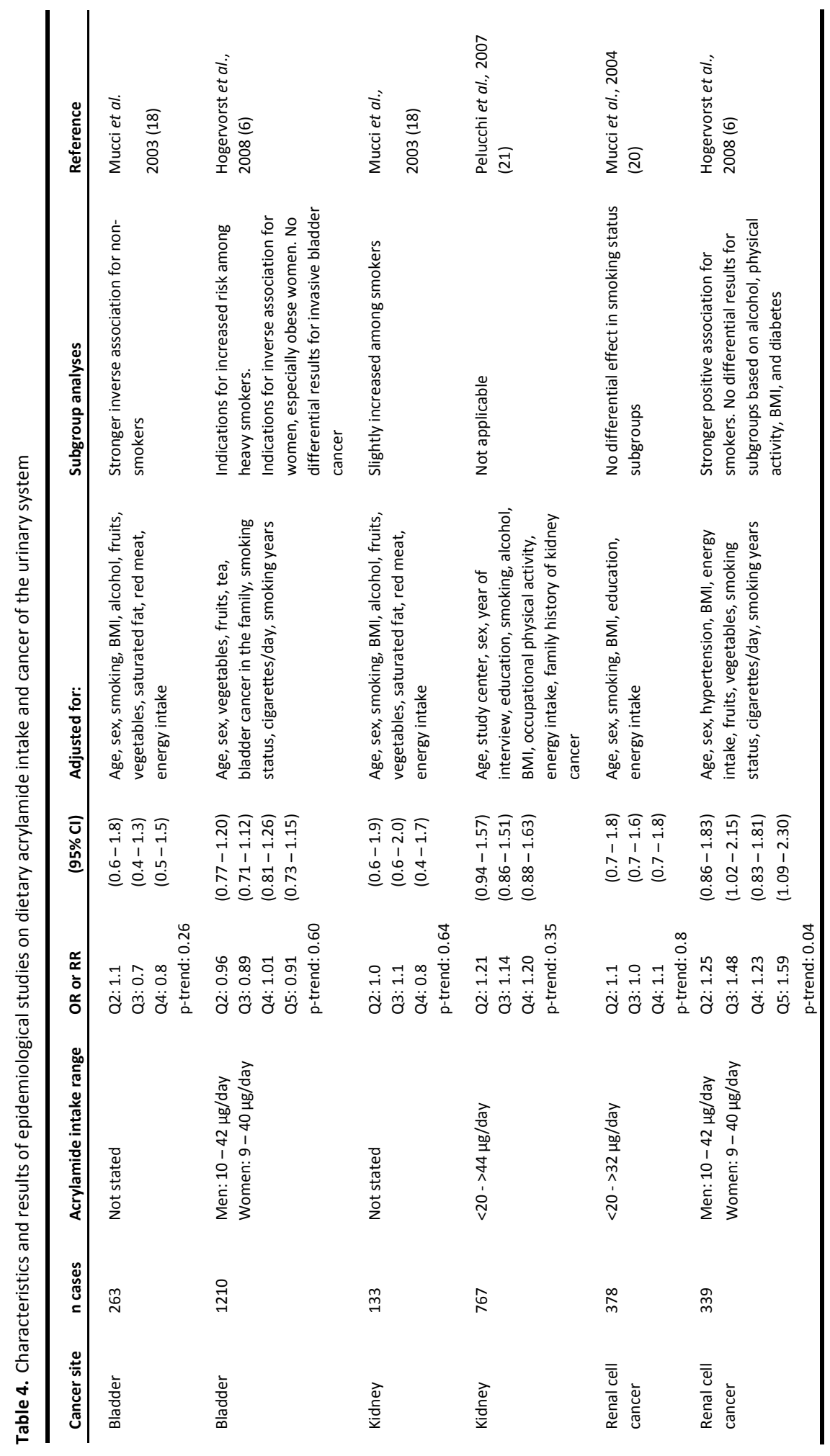


dietary acrylamide intake, which was strongest for adenocarcinoma. The association was stronger after elaborate statistical adjustment for smoking and was also present in neversmoking women.

\section{Other sites and total cancer}

In Table 6, the results of the studies on various organs and total cancer are presented.

Both thyroid (22) and brain cancer (24) were studied only once, in a prospective cohort study and no association was observed. Especially for thyroid cancer, the statistical power was probably very low and larger studies are needed to study this association.

One study investigated total cancer risk associated with dietary acrylamide intake (Hogervorst, unpublished results). There was no association with dietary acrylamide intake, neither for men, nor for women, and also in the highest decile of acrylamide intake no increased risks were observed.

\section{Summary of the epidemiological studies}

There are some epidemiological indications that dietary acrylamide might cause cancer in humans, and the indications are strongest for postmenopausal endometrial and ovarian cancer.

Two prospective cohort studies have observed some indications for a positive association with postmenopausal receptor-positive breast cancer risk, but one more strongly in smokers and the other only in never-smokers and not statistically significant (only after adjustment for consumption of cookies).

Further, a positive association was observed with renal cell cancer risk in one study, and a positive association with oral cavity cancer risk in non-smoking women (although based on few cases) in the same cohort study.

Interestingly, there are indications for inverse associations with lung cancer risk in women (statistically significant), advanced prostate cancer risk in never-smoking men, bladder cancer risk in women, and oro- and hypopharyngeal cancer risk in men.

However, these associations need to be investigated in other studies to be able to be more confident about which are true associations and which were chance findings.

\section{Validity of the epidemiological findings}

With regard to acrylamide carcinogenicity research in humans, there have only been observational studies, because human intervention studies are considered both unethical and unfeasible. Observational epidemiological studies have drawbacks compared with intervention studies, but these drawbacks can to a great extent be resolved in the design and analysis phases of the studies.

Observational studies are susceptible to confounding, which means that the effect of the independent variable of interest is entangled with the effect of a co-occurring independent variable that is a risk factor for the endpoint under study. The effects can be disentangled by restricting the analyses to subgroups (e.g. never-smokers to preclude confounding by smoking) or by taking the other known risk factors into account and adjusting for them in the analysis phase, using multivariable models. All of the epidemiological studies 


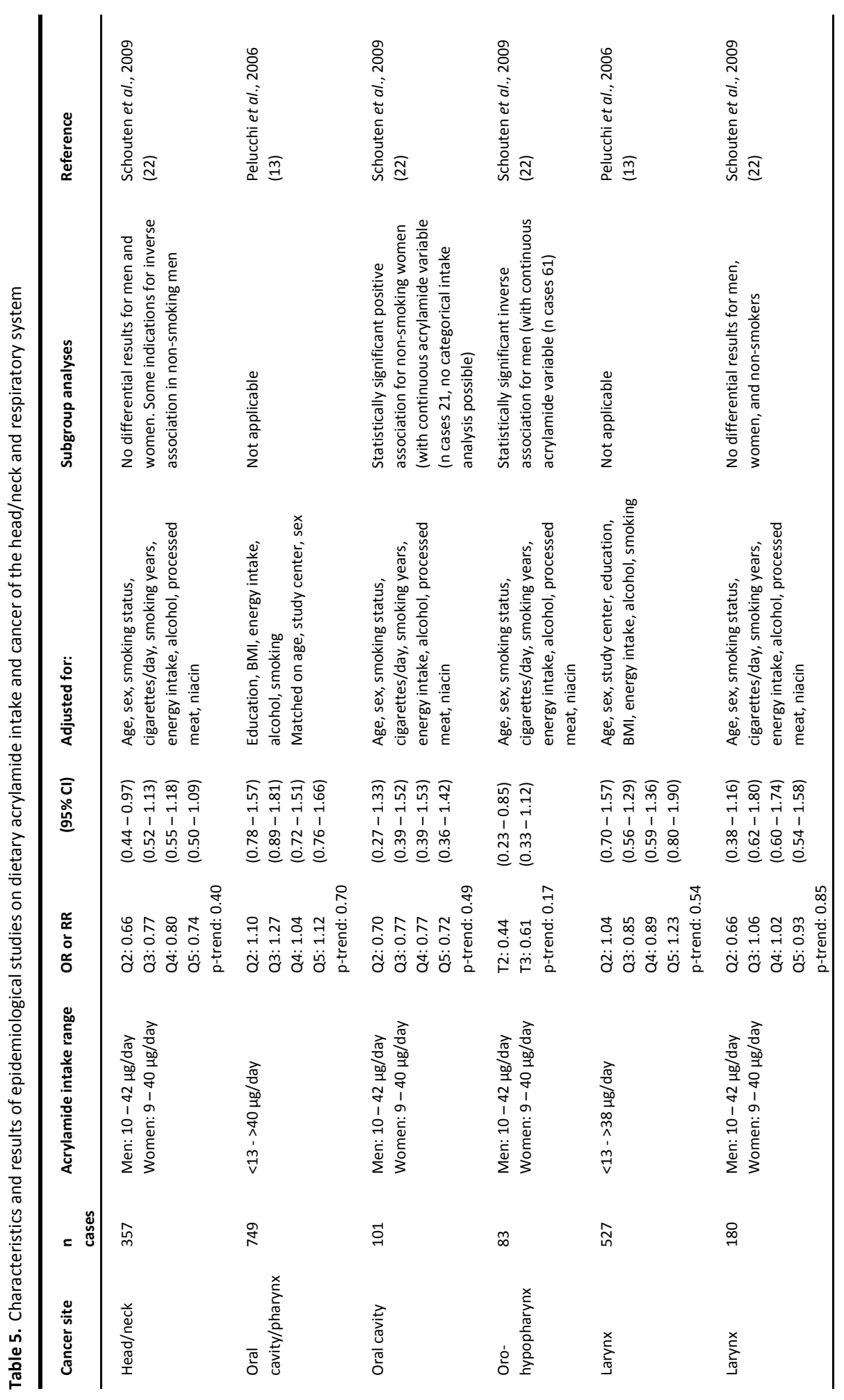




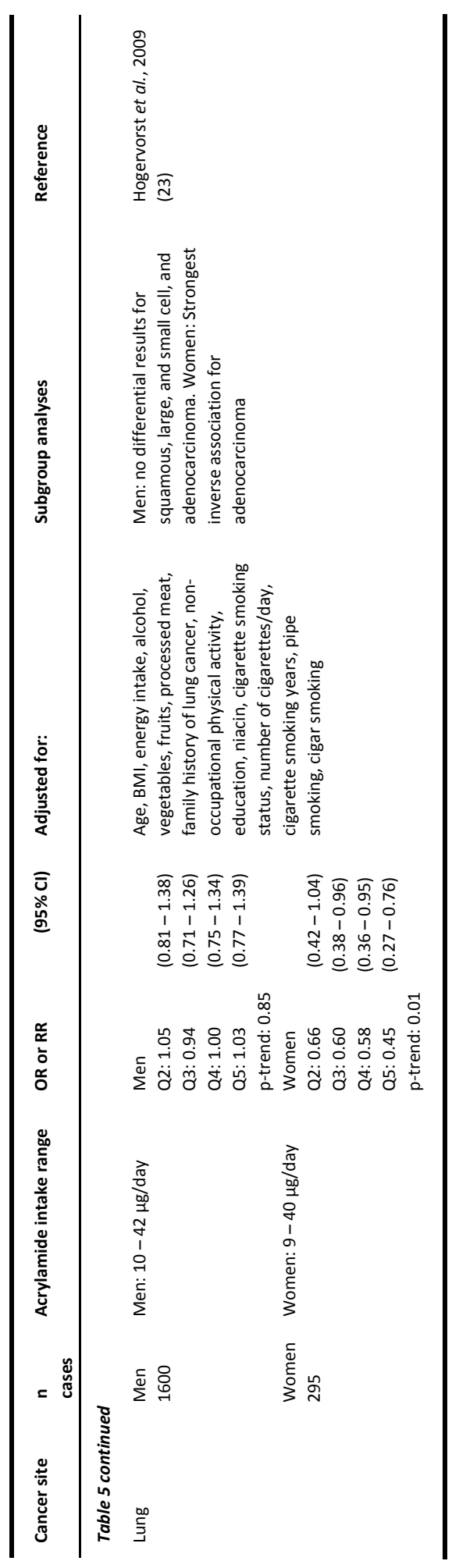




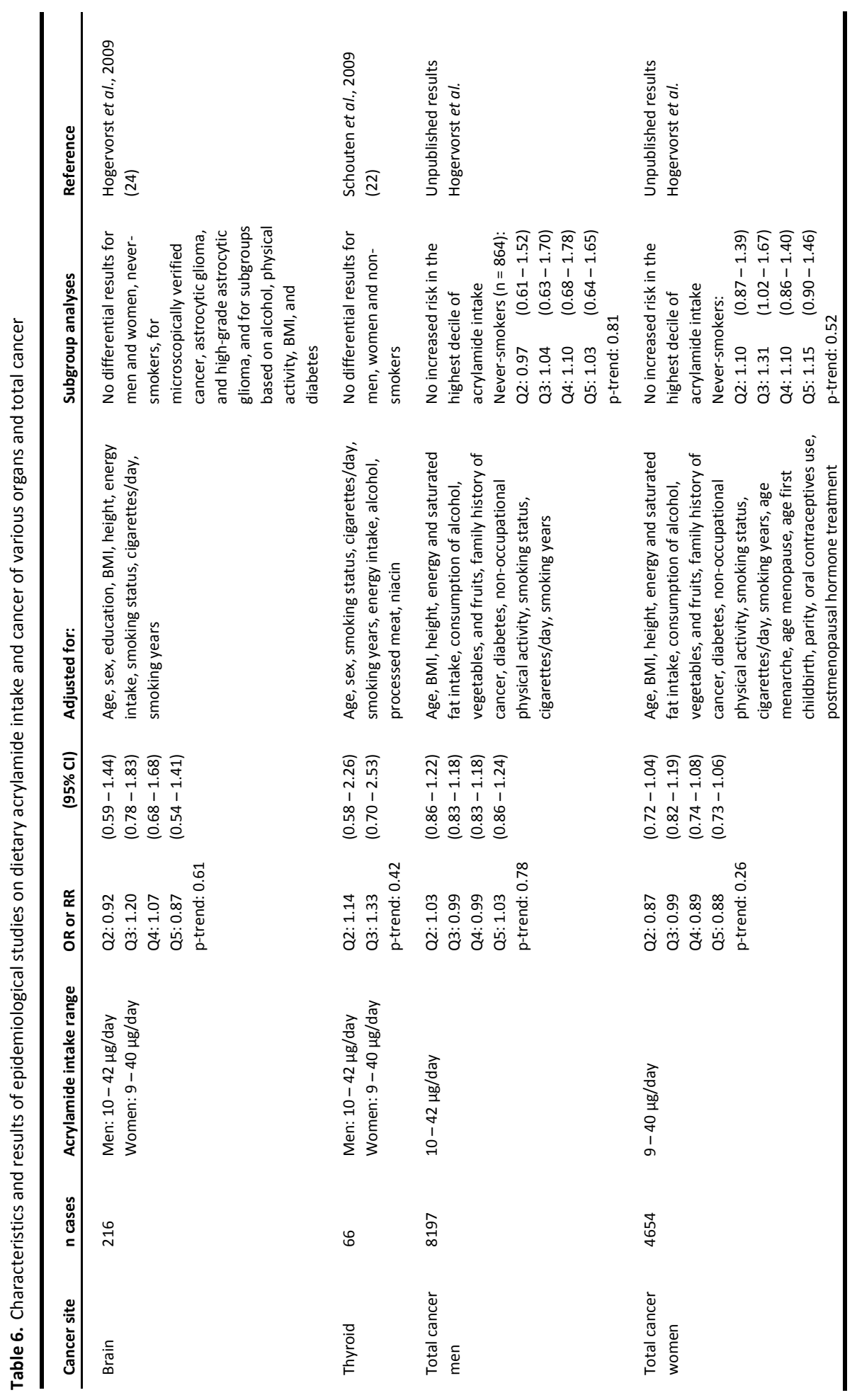


discussed in this review have elaborately adjusted for confounders, but in the study of Pelucchi et al. (13), no attention was paid to other food-related variables, such as fibre, saturated fat, and carbohydrates, which may have confounded their results due to their correlation with acrylamide in the diet. In all the other studies, several food-related variables were checked for their confounding potential. In addition, Hogervorst et al. $(4,6$, $17,23,24)$ and Pedersen et al. (15) have investigated the effect of adjusting for the main acrylamide-contributing food sources in their study population, to see if the effect was attributable to acrylamide itself or to some other characteristic of the individual acrylamide-containing foods. Their conclusion was that the observed associations were attributable to acrylamide itself, because the relative risks of acrylamide did not change importantly or got stronger after adjustment for the specific foods. Because confounders are usually measured imperfectly, which leads to residual confounding, it is important to see what happens to the acrylamide risk estimates when they are adjusted for confounders. To this end, epidemiological researchers should report both unadjusted and multivariableadjusted results. The studies that observed associations between acrylamide intake and cancer risk all showed unadjusted and multivariable-adjusted results (4-6) and the results became stronger after adjustment, which is reassuring, because it indicates that residual confounding by the covariables is probably not the explanation for the observed acrylamide-associated associations. Confounding by variables not adjusted for is still possible, but as already said, especially the later studies checked the confounding potential of most known risk factors.

Interestingly, significant associations (both positive and inverse) are predominantly found in later studies, which are in majority prospective cohort studies. Prospective cohort studies in general are devoid of selection bias and exposure information bias, which may be problems in case-control studies. These biases may obscure true associations. Another potential explanation is that the studies differ with regard to which are the most important acrylamide-contributing foods in the study populations. Some foods show less variation in acrylamide concentrations or may be easier to report on in an FFQ than other foods, which reduces the potential for misclassification. Some studies have included both questionnaire-derived information on frequency and portion size of consumption of acrylamide-containing foods, whereas other studies have used only frequency information from the FFQ, and combined this with age-specific or general population-based portion sizes. This latter approach entails more potential for misclassification of the acrylamide intake. There are also differences in the performance of the FFQs that are used, in terms of reproducibility and validity (see Table 1 ).

Further, the studies that have observed associations between acrylamide intake and cancer risk have had better exposure assessment methods (more extensive FFQs, frequency and portion size data, large national databases of acrylamide in foods, haemoglobin adduct data).

In conclusion, the later (mostly prospective cohort) studies were better suited to study the association between dietary acrylamide intake and cancer risk, which increases the likelihood that the observed associations are valid and causal. 


\section{Review of rodent carcinogenicity studies}

The search of articles was conducted through Pubmed, using the search terms acrylamide and cancer combined.

The results of the rodent studies on acrylamide carcinogenicity are summarised in Tables 7 through 9 . There have been two chronic rat carcinogenicity studies on Fischer 344 rats so far (Tables 7 and 8), and another study from the National Toxicology Program is currently underway (7). The daily doses tested in those two studies ranged from twenty times to six thousand times the amount that humans in industrialised countries generally ingest through their diets (on average $0.5 \mu \mathrm{g} / \mathrm{kg}$ body weight per day).

The first study (Table 7) showed increased tumour incidence for mammary gland, central nervous system, thyroid gland-follicular epithelium, oral tissues, uterus, benign adenoma of the pituitary gland, and clitoral gland for female rats, and benign thyroid gland adenoma, oral tissue epithelial hyperplasia, benign adrenal gland pheochromocytoma, and scrotal mesothelium for male rats (27).

In the second study (Table 8 ), these results were reproduced only for thyroid glandfollicular epithelium in both males and females, for scrotal mesothelium in males, and for mammary gland adenocarcinomas and fibroadenomas in females (28). A possible explanation for the inconsistencies is that a virus infection occurred in the rats in the first study (28).

Three studies were done with mice (Table 9); one with male and female A/J mice (sensitive model for lung adenomas) and female SENCAR mice (sensitive model for skin papilloma) (29), one with female Swiss-ICR mice (sensitive to chemical carcinogens for induction of both lung adenomas and skin papillomas) (30), and one with female SENCAR, $B A L B / C, A / J$ and Swiss-ICR mice (31). In the first study (29), acrylamide given by gastric intubation and intraperitoneal injection caused lung adenomas in male and female $A / J$ mice, and was a tumour initiator (not a complete carcinogen) in the skin of female SENCAR mice. In the female Swiss-ICR mice (30), acrylamide caused adenomas and carcinomas in the lungs, and squamous cell adenoma and carcinomas in the skin, but the latter was again dependent on TPA-induced promotion. In the third study with a single intraperitoneal acrylamide administration (31), the skin tumour effect in the SENCAR mice was reproduced (and lung adenoma incidence was also statistically significantly increased in SENCAR mice), but the lung tumour effect in ICR and A/J mice was not reproduced (31). No significant effects were seen in the BALB/c mice. Preliminary results of the NCR/NTP showed increased incidence of liver, lung and harderian gland tumours in mice, but the definitive list may include more sites (7).

\section{Cancer site concordance for acrylamide between rodents and humans}

Few cancer sites seem to correspond between humans and rodents. In both rats and humans, increased incidences of cancer of the mammary glands (in humans only for estrogen and-or progesterone receptor-positive breast cancer), oral cavity, and the uterus 


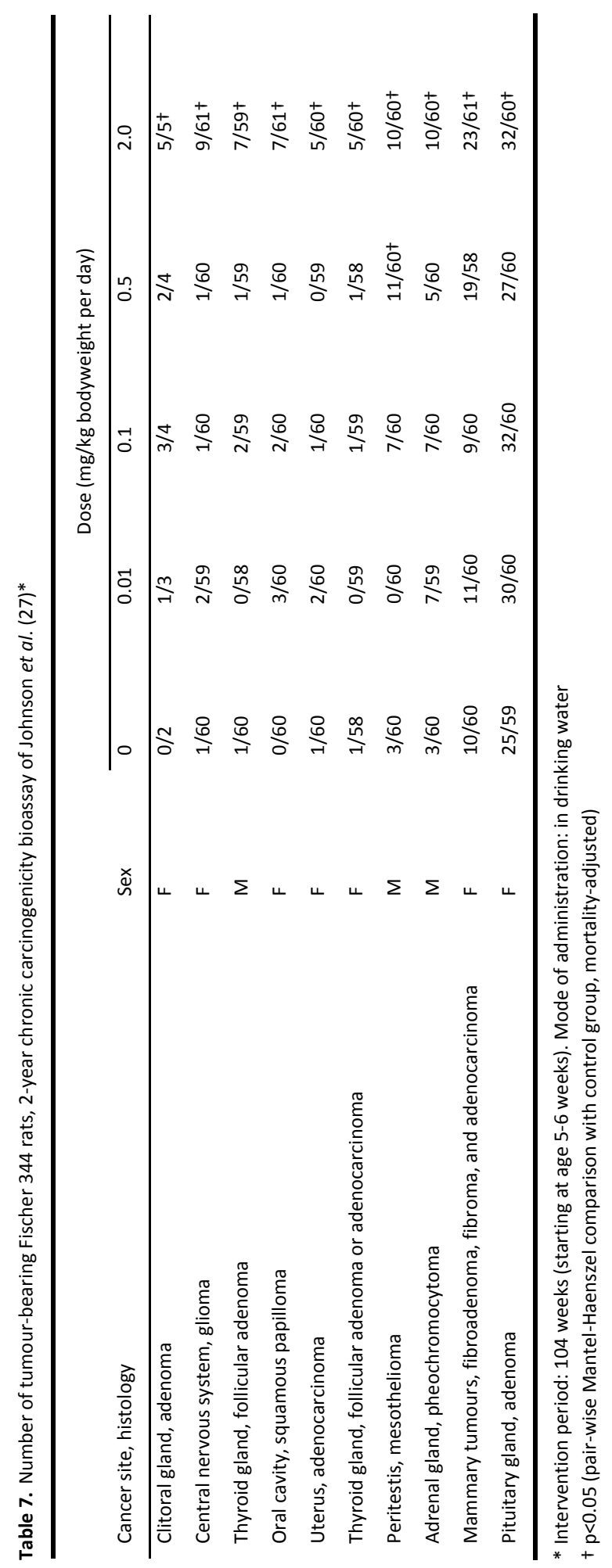




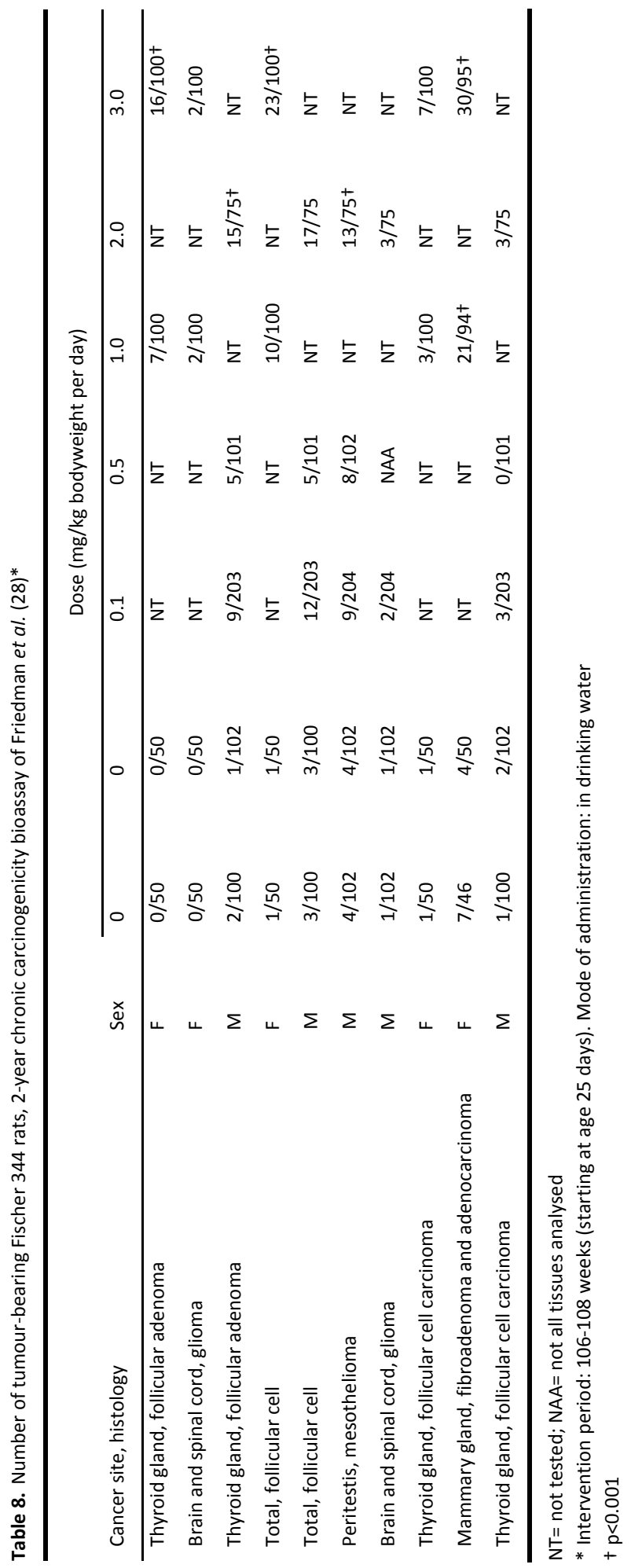




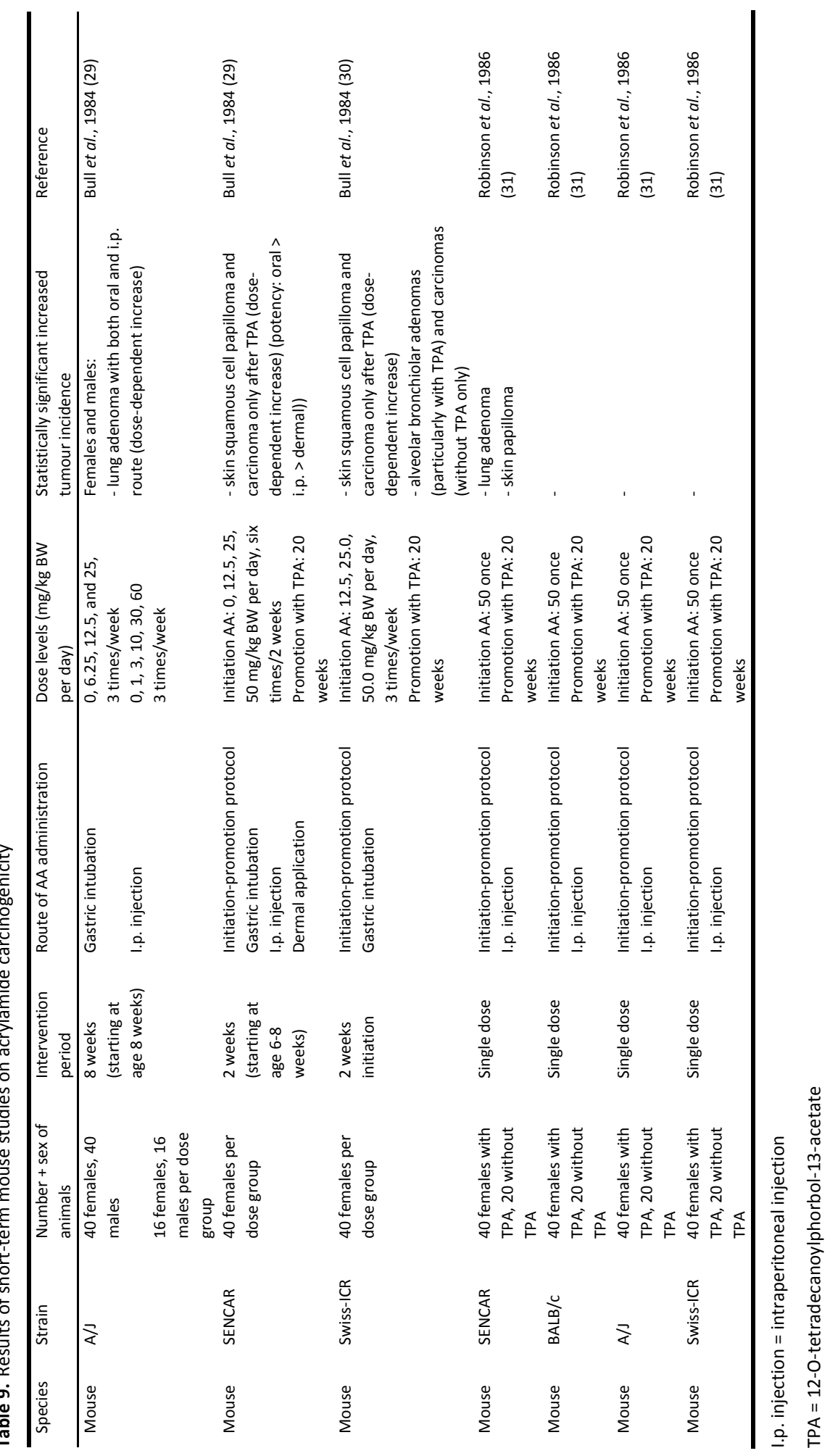


were observed, the latter two in only one of the two rat studies. Furthermore, the evidence for an association between acrylamide intake and receptor-positive breast cancer and oral cavity cancer risk in women is only weak.

There are some inconsistencies between animals and humans: in women, the incidence of lung cancer was decreased, whereas in female mice the incidence was increased. In humans, no increased incidences of thyroid and central nervous system tumours were observed, in contrast to what was observed in the rat studies, although thyroid and brain cancer have been investigated in only one epidemiological study. An increased incidence of kidney cancer was not observed in the rodents, whereas it was observed in one epidemiological study, particularly among smokers.

There have been no epidemiological studies yet on liver and skin cancer, so these cannot be compared with the animal results.

In humans, some indications for an inverse association were observed for lung cancer in women, bladder cancer in women, prostate cancer and oro- and hypopharynx cancer in men. In the animal studies, no attention was paid to any decrease in cancer incidence in response to exposure to an agent, so the inverse associations in humans cannot be compared with animals.

\section{Comparison of acrylamide cancer risk derived from rodents and risk observed in humans}

Because of acrylamide's presumed genotoxic mechanism of carcinogenesis, linear extrapolation from high chronic doses in rats to low chronic doses in humans is applied in acrylamide risk assessment. Acrylamide risk assessment is based on the chronic rat carcinogenesis assays, because the studies in mice were short-term studies that are less representative of the chronic dietary exposure conditions for humans. Further, it is unlikely that the observed increased skin and lung cancer incidence in mice has relevance for humans, because the inbred mice that were used are specifically sensitive to skin and lung cancer induction (28).

The outcome of the extrapolation from the rat data is that an intake of $1 \mu \mathrm{g} / \mathrm{kg}$ body weight per day would correspond to a relative risk of cancer in humans of between 1.015 and 1.05 compared with mean acrylamide intake (depending on which cancer slope factor is used) (32), which is around $0.5 \mu \mathrm{g} / \mathrm{kg}$ body weight per day in the Netherlands (2). In a recent publication, Dourson et al. have decoupled the proposed contribution of the mutagenic action and the thyroid growth-stimulating action of acrylamide to thyroid tumour induction in rats, using a probit model for risk assessment, which enables deriving a dose-response relationship for different modes of action combined (33). The cancer slope factor at low doses that they derived would lead to an even considerably lower relative risk than the relative risk mentioned earlier. Detection of relative risks in the order of magnitude mentioned above would already require unfeasibly large epidemiological studies. However, the relative risks observed in epidemiological studies for endometrial, ovarian and renal cell cancer are at least tenfold to a hundredfold higher than what would 
have been expected from the animal studies. Although these epidemiological results still need confirmation from other epidemiological studies, they indicate that acrylamide risk extrapolation based on the rat data might underestimate human cancer risk. This underestimation might be due to several factors that are discussed below.

Some may argue that the observed acrylamide risk estimates in the epidemiological studies are overestimations of the true risk for humans, because concurrently with acrylamide, people consuming heat-generated foods are exposed to a number of other carcinogenic substances in those foods, e.g. heterocyclic aromatic amines (HAAs), furans, and furan-derivatives, such as 5-hydroxymethylfurfural (HMF), that contribute to the magnitude of the observed effects of acrylamide. However, the reaction in which acrylamide is generated heavily relies on specific conditions, such as the presence of asparagine, whereas HAA formation for example depends on other precursors, such as phenylalanine and creatinine (34). Thus, across the span of a whole diet, the correlation between acrylamide and HAAs is probably limited and there seems little potential for confounding. For HMF this has been clearly shown, as no correlation between the estimated dietary intake of HMF and acrylamide was observed in a recent study (35). On the contrary, it is likely that the true risks are still underestimated based on the observed risks in the epidemiological studies due to non-differential misclassification of the acrylamide intake, which attenuates the observed risks. In addition, probably not all of the acrylamide that is taken in through the diet is bioavailable (36). For this reason too, the true risk estimated by the use of a food frequency questionnaire would be underestimated.

Another possible explanation for the difference between the predicted acrylamide effect size based on linear extrapolation from the rat data and the observed human data is that the cancer sites in animals may have little relevance for the human situation. Mammary fibroadenomas are the most common spontaneously occurring tumours in aged female F344 rats. It has been proposed that acrylamide enhances the age-related disruption of the hormonal status in female rats. The tunica vaginalis mesotheliomas of the male rat testes were considered to be secondary to the spontaneous occurrence of Leydig cell tumours, which are highly prevalent in the male F344 rat. High-dose chronic administration of acrylamide most likely exacerbates hormonal dysfunction, leading to Leydig cell tumours. Because both tumour types appear as specific malignancies of ageing F344 rats, it was suggested that the modes of action are not relevant for humans $(7,37)$. Human breast cancer is not of the fibroadenoma type, but mainly of the ductal (85\%) or lobular (15\%) adenocarcinoma type, whereas Leydig cell tumours in men are very rare. It is also unlikely that the increased incidence of thyroid tumours in rats is relevant to the human situation. It is a common type of cancer in rats, but rare in humans. In rats, thyroid tumours are probably caused by thyroid growth or function stimulating agents, not by genotoxic agents. Rodents are much more sensitive for disturbance of the thyroid gland function than man is, because they lack thyroid-binding globulin that in humans regulates the level of thyroid hormones in the circulation (38). In addition, thyroid hormone turnover is much higher in rodents than in man (37). The underlying mode of induction or promotion for all three rat cancer sites is probably disturbances of hormonal balances (33, 37). If the acrylamide-induced tumours in rats are indeed caused by hormonal distur- 
bances, then according to some the dose-response relationship for rats probably has a threshold below which no effects occur $(39,40)$. In that case, detectable increased cancer risks at the low dietary doses that humans are exposed to would have been expected even less than with a linear, non-thresholded, extrapolation.

There have not been any epidemiological studies in which it was investigated whether acrylamide induces hormonal changes in humans at the levels of general dietary exposure. However, the total body of evidence from the epidemiological acrylamide studies does point toward a hormonal carcinogenic effect (which is discussed in the next section). The fact that increased risks in humans were not expected based on the extrapolation from rat data might imply that the dose-response relationship for rats cannot be extrapolated to humans, even though both in rats and humans hormonal effects might be driving the carcinogenic effect.

Considering that the toxicokinetic properties of acrylamide in rats and humans seem reasonably comparable (41), apart from the approximately twofold more efficient conversion of acrylamide to glycidamide in rodents, reasons for the difference in risk magnitude may be a different type of toxicodynamic (hormonal) effect at the high rat dose compared with the low human dose or an entirely different type of toxicodynamic (hormonal) effect due to species differences. Chronic carcinogenicity assays in animals have a high sensitivity, which is shown by the fact that all human carcinogens that have been tested adequately have shown to be carcinogenic in animal studies as well (42). However, it is questionable whether it is the same mode of action in the laboratory animals and humans that underlies the carcinogenic action for the substances that were carcinogenic in both species.

In their paper on the mechanisms of non-genotoxic carcinogenesis and human hazard assessment, Silva Lima et al. discuss that while extrapolation from animal data to humans for genotoxic compounds is relatively straightforward, extrapolation for non-genotoxic compounds requires in depth understanding and characterisation of the mechanisms behind the carcinogenic activity (38). For hormonally acting non-genotoxic compounds, they indicate that the situation is often very complex and species-specific. In a study by Olson et al., comparison of hazard identification based on animal and human data for 150 compounds showed that concordance between human and animal toxicity was highest for effects on the gastrointestinal, hematological and cardiovascular systems, but lowest for cutaneous, hepatic, and endocrine effects (43).

There are several other examples of carcinogens for which risks for humans have been underestimated based on animal data, e.g. vinyl chloride, benzidine, benzene and butadiene $(44,45)$.

\section{Hormonal pathway in acrylamide carcinogenesis?}

There are several indications that acrylamide is genotoxic (both mutagenic and clastogenic) in vitro and in vivo, mainly after metabolisation to glycidamide, recently reviewed by Besaratinia et al. and Exon $(40,46)$, and discussed in Chapter 1 of this thesis. 
The theory of a hormonal pathway, perhaps occurring on top of a genotoxic action, was first suggested because of the predominantly hormonally sensitive sites at which cancer occurred after administration of acrylamide in animal studies $(27,28)$. Furthermore, the distribution pattern of glycidamide DNA adducts over the tissues did not match the tissue pattern of carcinogenesis in animals (47), indicating that genotoxicity may not be the (or not be the only) relevant cancer-causing characteristic of acrylamide. Recently, Dourson et al. compared time windows and dose-response relationships for genotoxic effects and thyroid tumour occurrence due to acrylamide in rats, and concluded that genotoxicity cannot be the only mechanism by which acrylamide causes thyroid tumours in rats (33). They infer this from the fact that dose-response curves for acrylamide-induced thyroid tumours in rats do not track those of acrylamide-induced genotoxicity in various cell types from various species, but that thyroid tumours are already induced at lower doses than genotoxic effects are. They put forward the hypothesis that acrylamide has thyroid-growth stimulating effects which might be driving thyroid tumour induction at high acrylamide dose levels. At low doses, such as those that humans are exposed to through diet, genotoxicity may be driving thyroid tumour induction.

Studies with rats show varying results for the role of acrylamide in the disruption of endocrine systems. Acrylamide was shown to influence levels of prolactin, testosterone, estrogen, and progesterone (48-50), and thyroid hormones in female rats $(49,51)$, but in a study with male rats no clear influence of acrylamide on the hypothalamus-pituitarythyroid axis was observed (39). Park et al. have found a synergistic effect of acrylamide and estrogen on morphological transformation of Syrian Hamster Cells (SHE), an endpoint that is widely used in studies on chemical carcinogenesis (38). A recent in vitro study on glycidamide-induced gene expression in human breast and colon cancer cells showed upregulation of genes that catalyse the conversion of inactive androgen and estrogen precursors to active forms, such as testosterone, and $17 \beta$-estradiol, although the authors indicate that this happened at high doses only, which are not reached with human dietary intake (39).

The total body of current epidemiological evidence for dietary acrylamide carcinogenicity discussed previously in this paper suggests that the carcinogenic pathway involves sex hormonal systems. The positive associations between dietary acrylamide intake and endometrial and ovarian cancer risk give the strongest suggestion for this, because established risk factors for these cancers are predominantly related to hormonal exposures (5257). Renal cell cancer has been positively associated with estrogens, both in animals and in humans (58-60). In prospective cohort studies, indications were found for an inverse association between reproductive factors and bladder cancer, in the form of an inverse association between age at menopause and bladder cancer and between lifetime years of ovulation and bladder cancer (45). In addition, both rodent and human bladder cells express estrogen receptors and estrogens inhibit bladder carcinogenesis in rodents (45). Furthermore, prostate cancer, for which indications of an inverse relationship with acrylamide intake were found, is a hormonally influenced type of cancer. In a prospective cohort study, in which the risk ratios for several hormones were mutually adjusted for each other and for sex hormone-binding globulin, testosterone was positively associated 
with the risk of prostate cancers, whereas estrogens were negatively associated with the risk of prostate cancer (61). Several lines of evidence indicate sex hormonal involvement in lung cancer as well. Normal human lung tissue and lung tumour tissue contain estrogen (especially estrogen receptor- $\beta$ (ER- $\beta)$ ), progesterone and androgen receptors, and the expression of the receptors differs between men and women and between lung cancer subtypes (62-64). Adenocarcinomas, e.g., express ER- $\beta$ to a higher extent than squamous cell carcinoma (63). Several epidemiological studies have looked at the association between postmenopausal hormone treatment and female lung cancer risk, and the majority indicates a protective effect of postmenopausal hormone treatment $(65,66)$. Within the NLCS study, in which positive associations were observed between acrylamide intake and endometrial, ovarian, and renal cell cancer, and an inverse association for female lung cancer risk (and non-statistically significant associations for female bladder cancer (inverse), hormone receptor-positive breast cancer (positive) and prostate cancer (inverse), statistically significant associations between several hormonal factors and female lung cancer risk were observed (23). Interestingly, the associations for postmenopausal hormone treatment, oral contraceptives, and BMI were opposite to those with endometrial and ovarian cancer, for which positive associations with acrylamide exposure were observed in the same cohort study (4).

Based on all these observations, we hypothesise that acrylamide may alter hormonal balances or systems in such a way that it decreases lung cancer risk in women (and maybe also female bladder cancer and prostate cancer risk), and increases endometrial, ovarian, renal cell, and possibly estrogen and or progesterone receptor-positive breast cancer risk. Genotoxicity as the often-presumed main mode of action cannot explain the observed inverse associations that, however, still have to be corroborated.

\section{How could this hormonal pathway work?}

As yet, one can only speculate on how acrylamide might disturb sex hormonal balances in humans. Acrylamide has been shown to bind to many proteins (67), among which may be proteins related to maintenance of hormonal balances. Acrylamide alkylates sulfhydryl (thiol) groups (68), and cysteine is the only amino acid that contains these groups.

The human estrogen receptor (ER) contains highly conserved cysteine-rich areas in the ligand-binding domain (69), which acrylamide thus might bind to. Interestingly, the pattern of organs for which positive or inverse associations were seen with acrylamide in the epidemiological studies appears to correspond to the pattern of organs in which mainly ER- $\alpha$ or ER- $\beta$ is expressed or to the function that either receptor type appears to have in the organs. In rats and humans, ER- $\alpha$ is primarily expressed in the uterus, kidney, liver and heart, whereas ER- $\beta$ is the most abundant receptor in the lung, prostate, bladder, gastrointestinal tract, and hematopoietic and central nervous systems $(70,71)$. Co-expression of the two receptor isoforms occurs in the mammary gland, ovary (epithelial cells), epidydemis, thyroid, adrenal gland, bone and certain brain regions (70-72).

Both isoforms have a small common set, but large distinct sets of genes that are transcribed upon ER activation, and thus the function of these receptor isoforms differs 
(73). Unlike ER- $\alpha, E R-\beta$ is thought to have a protective role against breast cancer in breast tissue, modulating proliferation and invasion of breast cancer cells (73). ER- $\beta$ is lost in a majority of breast tumours, probably by promoter methylation (73). In the ovaries, ER- $\beta$ is mainly localised in the granulosa cells (74), from which sex cord-stromal tumours develop, not epithelial tumours. In two epidemiological studies, acrylamide intake was associated with epithelial ovarian cancer risk. In the ovaries, ER- $\alpha$, and not ER- $\beta$, seems to be involved in the growth regulation of ovarian cancer in response to estrogen (53) and a loss of ER- $\beta$ expression or a decrease of the ratio between ER- $\beta$ and ER- $\alpha$ was observed in epithelial ovarian cancer cells as compared with normal tissues (73). In the prostate, ER- $\beta$ has antiproliferative and prodifferentiative effects (73). In different cancer cell lines, binding of $17 \beta$-estradiol to ER- $\alpha$ induced transcription of genes involved in cell cycle progression and inhibition of apoptosis, whereas binding of the hormone to ER- $\beta$ resulted in transcription of genes involved in caspase-3 activation, which leads toward apoptosis (75). ER- $\beta$ agonists were shown to have anti-inflammatory effects in rat models of arthritis and colon inflammation (76). Furthermore, ER- $\beta$ was shown to be an inhibitor of ER- $\alpha$ transcriptional activity and thus the relative expression level of both isoforms is an important determinant of cellular responses to estrogen agonists and antagonists (77). In conclusion, ER- $\alpha$ seems to act like an oncogene, whereas ER- $\beta$ seems to have tumour suppressor characteristics.

The ligand-binding domain of both isoforms is relatively well conserved, and thus many estrogens, like estradiol, or estrogen-like substances will bind to both receptors. The three-dimensional structure of the ligand-binding domain is also quite similar for both receptor types, although the binding cavity of ER- $\beta$ is approximately $20 \%$ smaller than that of ER- $\alpha$ (73). For a small molecule like acrylamide, this is probably not a limiting factor for ER- $\beta$ binding. If acrylamide is able to bind to and be an agonist for both receptors, the tissue distribution of and the specific effects of the two receptor isoforms could explain why acrylamide seems to have carcinogenic effects in some tissues, but anti-carcinogenic effects in other tissues.

Estrogen receptors from different species have been found to have differential ligandbinding preferences and binding affinities for estrogenic compounds (78-80), probably due to differences in the amino acid sequence in the ligand-binding domains. This is a possible toxicodynamic explanation for why humans may be more susceptible to acrylamide's carcinogenic effects than rats. Apart from activation of the estrogen receptor through direct binding to it, estrogenic substances can activate estrogen receptor mediated gene expression by modifying estrogen receptor phosphorylation (81).

Another candidate target for acrylamide may be sex hormone-binding globulin (SHBG), which is present in humans but not in rodents (82), that also has highly conserved cysteine-rich regions, which are required for steroid binding (83). Binding of acrylamide to this globulin could lead to altered free sex hormone levels in the circulation. This could be another possible toxicodynamic explanation for why humans might be more sensitive to acrylamide carcinogenesis than rats. 
Yet another possible mechanism by which acrylamide might exert hormonal effects could be by changing the metabolism of hormones through effects on the liver or by changing hormone levels by effects on the hypothalamus-pituitary axis $(33,84)$.

\section{Implications for public health}

As discussed in this review, acrylamide at current dietary exposure levels may raise the risk of postmenopausal endometrial and ovarian cancer. For these two types of cancer, the current level of evidence is strongest, because increased risks were observed in two large prospective cohort studies.

The level of evidence for a positive association with renal cell cancer, and oral cavity cancer in women, and estrogen and progesterone receptor-positive breast cancer risk is considerably lower, because these associations were either observed only once so far, with a small number of cases (oral cavity cancer), or in the case of receptor-positive breast cancer, in different smoking status subgroups in two studies.

Interestingly, at the same levels of dietary exposure, acrylamide might decrease the risk of lung cancer in women, although this was observed only once so far. The (statistically non-significant) inverse associations with advanced prostate cancer, bladder cancer in women, and oro- and hypopharyngeal cancer risk in men indicate that this may apply to more cancer types, but clearly more epidemiological studies are needed to corroborate or refute these findings. Unpublished analyses from the NLCS study for total cancer risk (see Table 6) suggest that acrylamide does not raise total cancer risk, which may be the result of averaging out of risk enhancing and risk lowering effects of acrylamide. Future epidemiological acrylamide research may corroborate that acrylamide might offer protection against some cancer types, which then would require a calculation of the net cancer risk, but perhaps more importantly of the net disability and quality-adjusted life years lost, conferred by dietary acrylamide intake.

In the mean time however, based on the precautionary principle, we are of the opinion that the current risk management approach to strive for acrylamide concentrations in foods that are as low as reasonably achievable (ALARA) should be maintained. Additionally, public health advice should be given by food safety authorities to limit intake of foods containing high concentrations of acrylamide, especially foods that do not have any known health benefits, such as French fries and potato crisps.

\section{Recommendations for future research}

The fact that there are now indications that dietary acrylamide might constitute a higher human cancer risk than anticipated from animal data, should in our opinion lead to more emphasis on epidemiological research into the acrylamide-cancer link in humans, rather than on experimental animal research. 
First and foremost, there is a need of more independent epidemiological studies with a high quality of acrylamide intake assessment investigating the link between acrylamide intake and cancer risk, preferentially prospective cohort studies. Especially endometrial, ovarian, hormone receptor-positive breast, renal cell, oral cavity, lung, bladder, and prostate cancer warrant further attention in this respect.

Within these studies, stratification based on sex and smoking status is important, because sex may be an effect modifier of the relationship between acrylamide intake and cancer risk, whereas smoking can be both an important confounder of the relationship, as discussed before, and an effect modifier.

It is important to investigate whether there are susceptible groups based on genotype of acrylamide-metabolising genes, exogenous and endogenous hormonal variables, and variables that influence CYP2E1 activity. This implies that the future studies have to be large. There are several large, ongoing prospective cohort studies that might be suitable for this (e.g. EPIC), which are listed by van den Brandt et al. (85).

With respect to exposure assessment, it would be informative to have both FFQ data (from an FFQ that measures both frequency and portion size of foods) and data on both acrylamide and glycidamide haemoglobin adduct levels.

To investigate the hypothesised hormonal mode of action of acrylamide in humans, correlations between acrylamide intake assessed by FFQ and biomarkers on one hand and biomarkers of the sex steroid hormonal system on the other hand can be investigated in observational studies, but preferentially in intervention studies (using normal portions of normally available acrylamide-containing foods). 


\section{References}

1. Dybing E, Sanner T. Risk assessment of acrylamide in foods. Toxicol Sci 2003;75:7-15.

2. Konings EJ, Baars AJ, van Klaveren JD, et al. Acrylamide exposure from foods of the Dutch population and an assessment of the consequent risks. Food Chem Toxicol 2003;41:1569-79.

3. Matthys C, Bilau M, Govaert Y, Moons E, De Henauw S, Willems JL. Risk assessment of dietary acrylamide intake in Flemish adolescents. Food Chem Toxicol 2005;43:271-8.

4. Hogervorst JG, Schouten L, Konings EJ, Goldbohm RA, van den Brandt PA. A Prospective Study of Dietary Acrylamide Intake and the Risk of Endometrial, Ovarian, and Breast Cancer. Cancer Epidemiol Biomarkers Prev 2007;16:2304-2313.

5. Olesen PT, Olsen A, Frandsen H, Frederiksen K, Overvad K, Tjonneland A. Acrylamide exposure and incidence of breast cancer among postmenopausal women in the Danish Diet, Cancer and Health Study. Int J Cancer 2008;122:2094-100.

6. Hogervorst JG, Schouten L, Konings EJ, Goldbohm RA, van den Brandt PA. Dietary acrylamide intake and the risk of renal cell, bladder, and prostate cancer. Am J Clin Nutr 2008;87:1428-38.

7. Summary Report EFSA scientific colloquium no. 11. Acrylamide carcinogenicity new evidence in relation to dietary exposure. 22-23 May 2008, Tabiano (PR), Italy.

8. Wilson KM, Balter K, Adami HO, et al. Acrylamide exposure measured by food frequency questionnaire and hemoglobin adduct levels and prostate cancer risk in the Cancer of the Prostate in Sweden Study. Int J Cancer 2009;124:2384-90.

9. Larsson SC, Hakansson N, Akesson A, Wolk A. Long-term dietary acrylamide intake and risk of endometrial cancer in a prospective cohort of Swedish women. Int J Cancer 2008;124:1196-1199.

10. Wilson KM, Mucci LA, Cho E, Hunter DJ, Chen WY, Willett WC. Dietary Acrylamide Intake and Risk of Premenopausal Breast Cancer. Am J Epidemiol 2009;169:954-61.

11. Schettgen T, Rossbach B, Kutting B, Letzel S, Drexler H, Angerer J. Determination of haemoglobin adducts of acrylamide and glycidamide in smoking and non-smoking persons of the general population. Int J Hyg Environ Health 2004;207:531-9.

12. Mucci LA, Sandin S, Balter K, Adami HO, Magnusson C, Weiderpass E. Acrylamide intake and breast cancer risk in Swedish women. Jama 2005;293:1326-7.

13. Pelucchi C, Galeone C, Levi F, et al. Dietary acrylamide and human cancer. Int J Cancer 2006;118:467-71.

14. Larsson SC, Akesson A, Wolk A. Long-term dietary acrylamide intake and breast cancer risk in a prospective cohort of Swedish women. Am J Epidemiol 2009;169:376-81.

15. Pedersen GS, Hogervorst JGF, Schouten LJ, Konings EJMG, R. Alexandra., van den Brandt PA. Dietary acrylamide intake and estrogen and progesterone receptor-defined postmenopausal breast cancer risk. Submitted.

16. Larsson SC, Akesson A, Wolk A. Long-Term Dietary Acrylamide Intake and Risk of Epithelial Ovarian Cancer in a Prospective Cohort of Swedish Women. Cancer Epidemiol Biomarkers Prev 2009;18:994-7.

17. Hogervorst JG, Schouten LJ, Konings EJ, Goldbohm RA, van den Brandt PA. Dietary acrylamide intake is not associated with gastrointestinal cancer risk. J Nutr 2008;138:2229-36.

18. Mucci LA, Dickman PW, Steineck G, Adami HO, Augustsson K. Dietary acrylamide and cancer of the large bowel, kidney, and bladder: absence of an association in a population-based study in Sweden. Br J Cancer 2003;88:84-9.

19. Mucci LA, Adami HO, Wolk A. Prospective study of dietary acrylamide and risk of colorectal cancer among women. Int J Cancer 2006;118:169-73.

20. Mucci LA, Lindblad P, Steineck G, Adami HO. Dietary acrylamide and risk of renal cell cancer. Int J Cancer 2004;109:774-6.

21. Pelucchi C, Galeone C, Dal Maso L, et al. Dietary acrylamide and renal cell cancer. Int J Cancer 2007;120:1376-7.

22. Schouten LJ, Hogervorst JGF, Konings EJM, Goldbohm RA, van den Brandt PA. Dietary Acrylamide Intake and the Risk of Head-neck and Thyroid Cancers: Results from the Netherlands Cohort Study. Am J Epidemiol, in press.

23. Hogervorst JGF, Schouten LJ, Konings EJM, Goldbohm RA, van den Brandt PA. Lung cancer risk in relation to dietary acrylamide intake. J Nat Cancer Inst 2009;101:651-62.

24. Hogervorst JGF, Schouten L, Konings EJM, Goldbohm RA, van den Brandt PA. Dietary acrylamide intake and brain cancer risk. 2009, Cancer Epidemiol Biomarkers Prev 2009;18:1663-6.

25. Moorman PG, Calingaert B, Palmieri RT, et al. Hormonal risk factors for ovarian cancer in premenopausal and postmenopausal women. Am J Epidemiol 2008;167:1059-69. 
26. Larsson SC, Kesson A, Bergkvist L, Wolk A. Dietary acrylamide intake and risk of colorectal cancer in a prospective cohort of men. Eur J Cancer 2009;45:513-6.

27. Johnson KA, Gorzinski SJ, Bodner KM, et al. Chronic toxicity and oncogenicity study on acrylamide incorporated in the drinking water of Fischer 344 rats. Toxicol Appl Pharmacol 1986;85:154-68.

28. Friedman MA, Dulak LH, Stedham MA. A lifetime oncogenicity study in rats with acrylamide. Fundam Appl Toxicol 1995;27:95-105.

29. Bull RJ, Robinson M, Laurie RD, et al. Carcinogenic effects of acrylamide in Sencar and A/J mice. Cancer Res 1984;44:107-11.

30. Bull RJ, Robinson M, Stober JA. Carcinogenic activity of acrylamide in the skin and lung of Swiss-ICR mice. Cancer Lett 1984;24:209-12.

31. Robinson M, Bull RJ, Knutsen GL, Shields RP, Stober J. A combined carcinogen bioassay utilizing both the lung adenoma and skin papilloma protocols. Environ Health Perspect 1986;68:141-5.

32. Hagmar L, Tornqvist M. Inconclusive results from an epidemiological study on dietary acrylamide and cancer. Br J Cancer 2003;89:774-5; author reply 775-6.

33. Dourson $\mathrm{M}$, Hertzberg $\mathrm{R}$, Allen $\mathrm{B}$, et al. Evidence-based dose-response assessment for thyroid tumorigenesis from acrylamide. Regul Toxicol Pharmacol 2008;52:264-89.

34. Totsuka Y, Nishigaki R, Sugimura T, Wakabayahsi K. The possible involvement of mutagenic and carcinogenic heterocyclic amines in human cancer. In: K. Skog and J. Alexander (eds.), Acrylamide and other hazardous compounds in heat-treated foods, Woodhead publishing in food science and technology, pp. 296327. Boca Raton: CRC Press, 2006.

35. Husoy $T$, Haugen M, Murkovic M, et al. Dietary exposure to 5-hydroxymethylfurfural from Norwegian food and correlations with urine metabolites of short-term exposure. Food Chem Toxicol 2008;46:3697-702.

36. Fuhr U, Boettcher MI, Kinzig-Schippers M, et al. Toxicokinetics of acrylamide in humans after ingestion of a defined dose in a test meal to improve risk assessment for acrylamide carcinogenicity. Cancer Epidemiol Biomarkers Prev 2006;15:266-71.

37. Shipp A, Lawrence G, Gentry R, et al. Acrylamide: review of toxicity data and dose-response analyses for cancer and noncancer effects. Crit Rev Toxicol 2006;36:481-608.

38. Silva Lima B, Van der Laan JW. Mechanisms of nongenotoxic carcinogenesis and assessment of the human hazard. Regul Toxicol Pharmacol 2000;32:135-43.

39. Bowyer JF, Latendresse JR, Delongchamp RR, et al. The effects of subchronic acrylamide exposure on gene expression, neurochemistry, hormones, and histopathology in the hypothalamus-pituitary-thyroid axis of male Fischer 344 rats. Toxicol Appl Pharmacol 2008;230:208-15.

40. Exon JH. A review of the toxicology of acrylamide. J Toxicol Environ Health B Crit Rev 2006;9:397-412.

41. Fennell TR, Friedman MA. Comparison of acrylamide metabolism in humans and rodents. Adv Exp Med Biol 2005;561:109-16.

42. Tomatis L, Melnick RL, Haseman J, Barrett JC, Huff J. Alleged misconceptions' distort perceptions of environmental cancer risks. Faseb J 2001;15:195-203.

43. Olson $H$, Betton $G$, Robinson D, et al. Concordance of the toxicity of pharmaceuticals in humans and in animals. Regul Toxicol Pharmacol 2000;32:56-67.

44. Legator MS. Underestimating risk for three important human carcinogens: vinyl chloride, benzene, and butadiene. Ann N Y Acad Sci 1997;837:170-5.

45. Sanner T, Dybing E. Comparison of carcinogen hazard characterisation based on animal studies and epidemiology. Basic Clin Pharmacol Toxicol 2005;96:66-70.

46. Besaratinia A, Pfeifer GP. A review of mechanisms of acrylamide carcinogenicity. Carcinogenesis 2007;28:519-28.

47. Segerback D, Calleman CJ, Schroeder JL, Costa LG, Faustman EM. Formation of N-7-(2-carbamoyl-2hydroxyethyl)guanine in DNA of the mouse and the rat following intraperitoneal administration of [14C]acrylamide. Carcinogenesis 1995;16:1161-5.

48. Ali SF, Hong JS, Wilson WE, Uphouse LL, Bondy SC. Effect of acrylamide on neurotransmitter metabolism and neuropeptide levels in several brain regions and upon circulating hormones. Arch Toxicol 1983;52:3543.

49. Mannaa F, Abdel-Wahhab MA, Ahmed HH, Park MH. Protective role of Panax ginseng extract standardized with ginsenoside Rg3 against acrylamide-induced neurotoxicity in rats. J Appl Toxicol 2006;26:198-206.

50. Uphouse LL, Nemeroff CB, Mason G, Prange AJ, Bondy SC. Interactions between "handling" and acrylamide on endocrine responses in rats. Neurotoxicology 1982;3:121-5.

51. Khan MA, Davis CA, Foley GL, Friedman MA, Hansen LG. Changes in thyroid gland morphology after acute acrylamide exposure. Toxicol Sci 1999;47:151-7. 
52. Salehi F, Dunfield L, Phillips KP, Krewski D, Vanderhyden BC. Risk factors for ovarian cancer: an overview with emphasis on hormonal factors. J Toxicol Environ Health B Crit Rev 2008;11:301-21.

53. O'Donnell AJ, Macleod KG, Burns DJ, Smyth JF, Langdon SP. Estrogen receptor-alpha mediates gene expression changes and growth response in ovarian cancer cells exposed to estrogen. Endocr Relat Cancer 2005;12:851-66.

54. Lukanova A, Kaaks R. Endogenous hormones and ovarian cancer: epidemiology and current hypotheses. Cancer Epidemiol Biomarkers Prev 2005;14:98-107.

55. Allen NE, Key TJ, Dossus L, et al. Endogenous sex hormones and endometrial cancer risk in women in the European Prospective Investigation into Cancer and Nutrition (EPIC). Endocr Relat Cancer 2008;15:485-497.

56. Kaaks R, Lukanova A, Kurzer MS. Obesity, endogenous hormones, and endometrial cancer risk: a synthetic review. Cancer Epidemiol Biomarkers Prev 2002;11:1531-43.

57. Akhmedkhanov A, Zeleniuch-Jacquotte A, Toniolo P. Role of exogenous and endogenous hormones in endometrial cancer: review of the evidence and research perspectives. Ann N Y Acad Sci 2001;943:296-315.

58. Nicodemus KK, Sweeney C, Folsom AR. Evaluation of dietary, medical and lifestyle risk factors for incident kidney cancer in postmenopausal women. Int J Cancer 2004;108:115-21.

59. Tanaka Y, Sasaki M, Kaneuchi M, Fujimoto S, Dahiya R. Estrogen receptor alpha polymorphisms and renal cell carcinoma--a possible risk. Mol Cell Endocrinol 2003;202:109-16.

60. Tanaka Y, Hirata H, Chen Z, et al. Polymorphisms of catechol-O-methyltransferase in men with renal cell cancer. Cancer Epidemiol Biomarkers Prev 2007;16:92-7.

61. Gann PH, Hennekens CH, Ma J, Longcope C, Stampfer MJ. Prospective study of sex hormone levels and risk of prostate cancer. J Natl Cancer Inst 1996;88:1118-26.

62. Lin P, Chang JT, Ko JL, Liao SH, Lo WS. Reduction of androgen receptor expression by benzo[alpha]pyrene and 7,8-dihydro-9,10-epoxy-7,8,9,10-tetrahydrobenzo[alpha]pyrene in human lung cells. Biochem Pharmacol 2004;67:1523-30.

63. Omoto Y, Kobayashi Y, Nishida K, et al. Expression, function, and clinical implications of the estrogen receptor beta in human lung cancers. Biochem Biophys Res Commun 2001;285:340-7.

64. Ishibashi H, Suzuki T, Suzuki S, et al. Progesterone receptor in non-small cell lung cancer--a potent prognostic factor and possible target for endocrine therapy. Cancer Res 2005;65:6450-8.

65. Rodriguez C, Spencer Feigelson H, Deka A, et al. Postmenopausal hormone therapy and lung cancer risk in the cancer prevention study II nutrition cohort. Cancer Epidemiol Biomarkers Prev 2008;17:655-60.

66. Kabat GC, Miller AB, Rohan TE. Reproductive and hormonal factors and risk of lung cancer in women: a prospective cohort study. Int J Cancer 2007;120:2214-20.

67. Park J, Kamendulis LM, Friedman MA, Klaunig JE. Acrylamide-induced cellular transformation. Toxicol Sci 2002;65:177-83.

68. Srivastava S, Sabri MI, Agrawal AK, Seth PK. Effect of single and repeated doses of acrylamide and bis-acrylamide on glutathione-S-transferase and dopamine receptors in rat brain. Brain Res 1986;371:319-23.

69. Aliau S, Mattras H, Richard E, Borgna JL. Cysteine 530 of the human estrogen receptor alpha is the main covalent attachment site of 11beta-(aziridinylalkoxyphenyl)estradiols. Biochemistry 1999;38:14752-62.

70. Matthews J, Gustafsson JA. Estrogen signaling: a subtle balance between ER alpha and ER beta. Mol Interv 2003;3:281-92.

71. Kuiper GG, Carlsson B, Grandien K, et al. Comparison of the ligand binding specificity and transcript tissue distribution of estrogen receptors alpha and beta. Endocrinology 1997;138:863-70.

72. Taylor AH, Al-Azzawi F. Immunolocalisation of oestrogen receptor beta in human tissues. J Mol Endocrinol 2000;24:145-55.

73. Zhao C, Dahlman-Wright K, Gustafsson JA. Estrogen receptor beta: an overview and update. Nucl Recept Signal 2008;6:e003.

74. Lau KM, Mok SC, Ho SM. Expression of human estrogen receptor-alpha and -beta, progesterone receptor, and androgen receptor mRNA in normal and malignant ovarian epithelial cells. Proc Natl Acad Sci U S A 1999;96:5722-7.

75. Acconcia F, Totta P, Ogawa S, et al. Survival versus apoptotic 17 beta-estradiol effect: role of ER alpha and ER beta activated non-genomic signaling. J Cell Physiol 2005;203:193-201.

76. Harris HA, Albert LM, Leathurby Y, et al. Evaluation of an estrogen receptor-beta agonist in animal models of human disease. Endocrinology 2003;144:4241-9.

77. Hall JM, McDonnell DP. The estrogen receptor beta-isoform (ERbeta) of the human estrogen receptor modulates ERalpha transcriptional activity and is a key regulator of the cellular response to estrogens and antiestrogens. Endocrinology 1999;140:5566-78. 


\section{Chapter 9}

78. Harris HA, Bapat AR, Gonder DS, Frail DE. The ligand binding profiles of estrogen receptors alpha and beta are species dependent. Steroids 2002;67:379-84.

79. Barrett JC. Mechanisms for species differences in receptor-mediated carcinogenesis. Mutat Res 1995;333:189-202.

80. Matthews J, Celius T, Halgren R, Zacharewski T. Differential estrogen receptor binding of estrogenic substances: a species comparison. J Steroid Biochem Mol Biol 2000;74:223-34.

81. Nilsson S, Makela S, Treuter E, et al. Mechanisms of estrogen action. Physiol Rev 2001;81:1535-65.

82. Reventos J, Sullivan PM, Joseph DR, Gordon JW. Tissue-specific expression of the rat androgen-binding protein/sex hormone-binding globulin gene in transgenic mice. Mol Cell Endocrinol 1993;96:69-73.

83. Wong AS, Lui WY, Hui IT, Lee WM. Rabbit sex hormone-binding globulin: expression in the liver and testis during postnatal development and structural characterization by truncated proteins. Int J Androl 2001;24:165-74.

84. Mei N, Guo L, Tseng J, Dial SL, Liao W, Manjanatha MG. Gene expression changes associated with xenobiotic metabolism pathways in mice exposed to acrylamide. Environ Mol Mutagen 2008;49:741-5.

85. van den Brandt P, Voorrips L, Hertz-Picciotto I, et al. The contribution of epidemiology. Food Chem Toxicol 2002;40:387-424. 


$$
x_{x}
$$


This Chapter contains a discussion of the most important findings on the association between acrylamide intake and cancer risk from the Netherlands Cohort Study on diet and cancer in light of some important strengths and weaknesses of the study design and the analyses. Furthermore, recommendations for future epidemiological research for the link between acrylamide and cancer are given. It does not contain a summary of the epidemiological and the experimental animal research on acrylamide and cancer that has been published so far or possible mechanisms of acrylamide carcinogenicity, as this was extensively discussed in Chapter 9.

As described in this thesis, statistically significant positive associations were observed between dietary acrylamide intake and the risk of postmenopausal endometrial cancer (in never-smokers), postmenopausal ovarian cancer, renal cell cancer (especially in long-term smokers), and a statistically significant inverse association for lung cancer in women. For these cancer types, the results are most solid, because they are based on considerable numbers of cases, and both the hazard ratio per increment of the continuous acrylamide variable and the trend across the categories of intake were statistically significant.

For some cancer types there were indications for an association, but the findings were less solid. In non-smoking women, there was a statistically significant positive association between acrylamide intake and oral cavity cancer risk, although based on only 21 cases. In men, acrylamide intake was significantly inversely associated with oro- and hypopharyngeal cancer risk when modeled as a continuous variable, but there was no clear doseresponse relationship across the tertiles of intake. Statistically non-significant associations were observed for estrogen and progesterone receptor-positive breast cancer in neversmoking women (positive), bladder cancer in women (inverse), and advanced prostate cancer in never-smoking men (inverse).

In Chapter 9, it was discussed that the observed risks are higher than those that were anticipated based on experimental animal carcinogenicity assays.

We will now discuss some of the most important issues that play a role in the judgment of the causality of these associations.

\section{Confounding}

Confounding is a potential problem in observational research, in contrast to in experimental research, where the exposure of interest and possible confounding variables are separated, because known and unknown confounding variables are in theory randomly distributed over the intervention groups. In observational research, confounding has to be controlled for in the design phase of the study by restriction of the selected study population or in the analysis phase by stratification or by multivariable-adjusted modelling. In our analyses, we have elaborately investigated the confounding potential of other risk factors for the specific cancer types.

For all the analyses, the variables that are most certainly known to be risk factors were included a priori in the statistical models. Further, other potential risk factors were identified from the literature, including the recently updated textbook on cancer epidemi- 
ology by Schottenfeld et al., in which the epidemiology for several cancer types is reviewed (1). These additional variables were checked for their confounding potential based on their influence on the risk estimate for acrylamide ( $>10 \%$ change of the HR for acrylamide). Additionally, we always checked the confounding potential of fibre, saturated fat, trans-unsaturated fatty acids, and carbohydrates, which may be confounders due to their correlation with acrylamide in the diet. Finally, we investigated the effect of adjusting for the main acrylamide-contributing food sources in our study population (Dutch spiced cake, coffee, cookies, French fries and potato crisps), to see if the association could be attributed to acrylamide itself or to some other characteristic of the individual acrylamidecontaining foods.

Because confounders are often measured imperfectly, which may lead to residual confounding, it is important to investigate the influence of adjusting for confounders on the acrylamide risk estimates. To this end, epidemiological researchers should show both unadjusted and multivariable-adjusted results in their reports. In our analyses, the statistically significant associations became stronger after adjustment. This indicates that residual confounding by the included covariables is probably not the explanation for the observed acrylamide-associated associations.

Although we checked for the confounding potential of many risk factors, confounding by unknown (and thus not taken into account) variables is still possible. However, the unknown factor would have to be a strong risk factor and strongly correlated to acrylamide intake to be able to explain a hazard ratio of 2 , such as observed for endometrial cancer in never-smoking women and ovarian cancer.

The HEATOX project has recently reported that preparing foods with heat can generate many possibly carcinogenic compounds, besides acrylamide: e.g. ethylcarbamate, furans, heterocyclic aromatic amines, hydroxymethylfurfural (HMF), polycyclic aromatic hydrocarbons, and nitrosamines (2). More research has to be done in order to investigate the correlation between dietary acrylamide intake and dietary intake of these other toxicants. The correlation, and thus the confounding potential, may be limited however, as discussed for HMF in Chapter 9.

From a methodological point of view, it is an advantage that acrylamide is present in different types of foods, e.g. coffee and potato crisps. Because of this, the correlations between acrylamide and other food components are not strong (the highest correlation between acrylamide and a long list of macro- and micronutrients was 0.44 (for niacin) in our study population), which makes it easier to separate the effect of acrylamide from that of other dietary factors and other heat-generated toxicants, but also from that of lifestyle factors. Thus, the potential for residual or unmeasured confounding is probably limited.

\section{Selection bias}

Selection bias due to selection of the study population is unlikely in cohort studies, since the entire population at the start of the study is disease-free, unlike in case-control stud- 
ies, where selection of the control population may introduce bias when the controls are not representative (in terms of exposure and/or other variables) for the source population of the cases. In addition, there may also be problems with the selection of cases. In cohort studies, selection bias can occur when participants are lost to follow-up and when this depends on exposure. However, in our study, the completeness of detection of cancer cases in the cohort was estimated to be at least $96 \%$ complete (3) and the follow-up of the subcohort was nearly $100 \%$ complete (only two male subcohort members (and later on only one) were lost to follow-up). Therefore, it is unlikely that selection bias has distorted our findings.

\section{Information bias}

\section{Food frequency questionnaire}

There are a number of sources of misclassification that can arise from the use of a food frequency questionnaire to estimate dietary acrylamide intake.

First, it is difficult to take into account the large variation in acrylamide concentrations within foods using questionnaires. Up to now single acrylamide values (means or medians) have been assigned to foods in studies using FFQs to assess dietary acrylamide intake. Perhaps some precision can be gained by inquiring about the brownness of foods (by giving answer options based on photos of foods with different degrees of brownness in the questionnaire).

Second, FFQs are often designed to measure long-term food intake, for which habitual food intake over extended periods (often a year) is queried. FFQ respondents are unlikely to be able to exactly report their average consumption of foods over a period, and this leads to misclassification as well. Although there is no direct data for acrylamide itself, the reliability and validity of the NLCS FFQ for acrylamide can be derived from nutrients that are correlated to acrylamide, namely carbohydrates and dietary fibre. As for validity, Spearman correlation coefficients between the FFQ and a 9-day dietary record method were 0.77 for carbohydrates and 0.74 for fibre. For the food groups potatoes, bread, and cakes and cookies, the correlation coefficients were $0.74,0.80$ and 0.65 , respectively (4). Through repeated annual administration of the NLCS FFQ to a random subsample of the cohort during the first five years of follow-up, it was shown that the correlation over all nutrients declined only by 0.07 on average. This indicates that, although the questionnaire was administered only once for the cases and subcohort, it characterises long-term food intake for over a period of at least five years (5). For coffee, which contributes importantly to acrylamide intake in this cohort, $73 \%$ of the subcohort members reported at baseline to drink the same amount of coffee as they did five years before.

Both types of misclassification mentioned above are non-differential, which means that they do not depend on disease status, because at the time of the exposure assessment the population under study was still disease-free. As a sensitivity analysis, we always checked whether the results changed importantly when the first two years of follow-up were excluded. This is important, because preclinical (still undiagnosed) disease at base- 
line may have caused dietary habits to change, which would then at the time of the measurement not be representative of long-term dietary habits. In our analyses, the results never changed importantly after exclusion of the first two years of follow-up.

Non-differential misclassification leads to attenuation of the true acrylamideassociated cancer risks. Thus, this type of misclassification makes it harder to observe true associations, and can therefore be an explanation when no associations are observed. It is not an explanation for observed associations, because the consequence of non-differential misclassification is attenuation of the true association. Thus, observed associations may in reality be stronger.

\section{Food frequency questionnaires versus acrylamide and glycidamide haemoglobin adducts}

There have been a few studies that investigated the correlation between dietary acrylamide intake as assessed by FFQ and acrylamide to haemoglobin or acrylamide and glycidamide to haemoglobin adducts (6-11). In some of these studies, the correlation was either low or absent $(6,7)$, and the authors of these papers are of the opinion that this is due to inadequacy of the FFQ to measure acrylamide intake, due to the sources of misclassification mentioned in the information bias section above. In this comparison, they use acrylamide to haemoglobin adducts as a kind of gold standard for measuring dietary acrylamide exposure. In other studies, modest correlations have been observed between FFQ data and haemoglobin adduct levels $(8,10,11)$.

The fact that these correlations are absent, low or modest is probably due to issues relating to both the FFQ (as discussed above) and the biomarker. Acrylamide adducts to haemoglobin can be used as a biomarker of exposure, but they represent the acrylamide exposure during the preceding three to four months only. It is conceivable that acrylamide haemoglobin adducts show seasonal variation, due to different consumption patterns in different seasons. The long half-life of haemoglobin and the fact that haemoglobin adducts are not repaired means that the measured adduct levels reflect a time-weighted average over the lifetime of the erythrocyte. Thus, similar levels of adducts can arise from a low exposure over an extended time period and from a high incidental exposure. This too impedes the comparison of the biomarker and the FFQ, because by nature the FFQ inquires about average exposure over an extended (usually a year) period. Furthermore, biomarkers are not specific with regard to the source of acrylamide. Thus, in biomarker studies of dietary acrylamide exposure, strict control for smoking (it is better to restrict to non-smokers) and passive smoking is of the utmost importance. Acrylamide and glycidamide haemoglobin adducts are expressed per gram of globin. This means that acrylamide exposure is standardised to the haemoglobin concentration in the blood. Thus, two persons with the same actual acrylamide intake may have different acrylamide haemoglobin adduct levels, dependent on their haemoglobin status. Factors that influence haemoglobin levels are plentiful: sex, age, smoking, alcohol intake, physical exercise, diet, etc. (12-14). Therefore, these variables should be taken into account when investigating the correlation between FFQ data and haemoglobin adduct data.

Finally, only in the study by Wilson et al., were FFQ data correlated to the sum of acrylamide and glycidamide haemoglobin adducts (10). This seems a better approach 
because this marker of exposure is less dependent on acrylamide metabolism than solely acrylamide haemoglobin adducts. However, the issues discussed above for acrylamide to haemoglobin adducts also apply to the sum of acrylamide and glycidamide to haemoglobin adducts.

In conclusion, there are many factors related to the biomarker too, that can reduce the correlation between FFQ data and acrylamide haemoglobin adduct level. Even with a hypothetical FFQ that would be able to perfectly assess dietary acrylamide intake, the correlation with the sum of acrylamide and glycidamide haemoglobin adducts will never be 1 .

\section{Etiologically relevant time window for exposure}

As discussed in Chapter 1 and 9, acrylamide could be effective in both the first phase of carcinogenesis, causing the initial gene mutations in critical genes in what is called the initiation phase, and in the promotion phase, causing cell proliferation of initiated cells due to a hormonal effect. As discussed before in this Chapter, the NLCS FFQ characterises long-term food intake for over a period of at least five years (15). Therefore, if acrylamide were to exert its effects primarily in the promotion phase of cancer, we have probably measured the acrylamide intake in at least a part of the etiologically relevant time window. However, it is uncertain whether the intake measured at baseline would still be sufficiently representative of the exposure at the time of tumour initiation, which is probably decades before the diagnosis of a tumour.

\section{Multiple comparisons}

Due to the prospective design and the size of the NLCS study, it was possible to study dietary acrylamide risk comprehensively: with the same methodology, we were able to investigate the link between acrylamide intake and many types of cancer.

There were two good reasons to do so: a) acrylamide reaches virtually every tissue due to its hydrophilicity and because it is a small molecule and b) just like in the rat studies, looking at many tissues and the possible pattern that emerges from the tissues that showed an increased cancer incidence, hypotheses about the mode of action of acrylamide carcinogenicity in humans can be derived. An important difference with the animal studies is that we also reported on inverse associations, whereas in animal studies no attention is paid to possible protective effects of toxicants. Inverse associations can be detected in animal studies too, since many tissues are pathologically examined, but they never seem to be statistically tested and reported on. This is a pity, because inverse associations can contribute to understanding of the mode of action also.

The drawback of studying many associations in epidemiology is that some of the observed statistically significant associations may be chance findings. This is equally applicable to the animal studies, and this is one of the reasons why acrylamide risk as- 
sessment is often based on the tumours that were increased in both rat studies: mammary gland, thyroid, and testis tumours. To be more confident that the findings in this thesis are not chance findings, it is needed to see them reproduced in other epidemiological studies.

For endometrial and ovarian cancer, positive associations have been observed in the prospective Nurses' Health Study as well (16), and that makes it less likely that these associations are due to chance. Also with respect to confounding, it is important that this other study, conducted in another population and with other main acrylamide food sources, has corroborated our findings. However, still other epidemiological studies should investigate these associations. For hormone receptor-positive breast cancer, two studies have observed some indications for a positive association, but these findings have to be interpreted cautiously because the associations were observed in opposing subgroups (smokers versus non-smokers).

For most of the observed associations in this study, there were indications of a monotonic dose-response relationship between the level of acrylamide intake and cancer risk, most clearly for ovarian cancer and lung cancer in women. It is unlikely that these are caused solely by chance.

\section{Biological plausibility}

In Chapter 9, we have proposed a possible mechanism that could explain the observed pattern of cancer risks in humans. Hormonal effects of acrylamide may be at the etiological basis of these cancers, with the type of estrogen receptor that is predominant in the tissue determining whether acrylamide exerts a risk enhancing or a protective effect. The statistically non-significant positive association with receptor-positive breast cancer risk in never-smokers, and the statistically non-significant inverse associations with bladder cancer risk in women and prostate cancer in never-smokers would also fit in this hypothesis.

\section{Advantages of the NLCS for studying the acrylamide-cancer link}

A great advantage in terms of validity is that the NLCS is a prospective cohort study, which is less susceptible to some types of bias than a case-control study is, as discussed in Chapter 1 (Study design section).

To obtain an intake estimate representative of our Dutch study population, we only used data on acrylamide concentrations in foods on the national market, and we incorporated individually reported portion sizes of foods in the intake assessment. Many of the other epidemiological studies did not use individually reported portion sizes.

It is common practice to assign a concentration of half the quantitation limit to foods that contain less than the quantitation limit, which may lead to overestimation of the acrylamide intake if the quantitation limit is high and the true acrylamide content of the 
food is low. In the period (2002-2005), in which the acrylamide analyses in foods that we used in our acrylamide intake assessment were done, the quantitation limit of the method decreased (17). This prompted us to reanalyse breads with the more sensitive method, which in the end rendered a considerably lower acrylamide concentration for bread (based on a value of half the quantitation limit). Studies that used data on acrylamide in foods that were analysed with a less sensitive method may have assigned too high an acrylamide concentration to foods that contained acrylamide below the limit of quantitation. This may result in considerable misclassification, especially when this is the case for foods that are eaten in great amounts, such as bread.

In the NLCS study population, there was a considerable range of dietary acrylamide intake, which increases the chance of detecting a true association. In the Swedish Mammography Cohort for instance, the range in acrylamide concentrations was lower than in our study; 34 in the highest and $16 \mu \mathrm{g} /$ day in the lowest category of intake versus 40 and 9 $\mu \mathrm{g} /$ day, respectively.

In some study populations, it may be easier to observe an association between dietary acrylamide intake and cancer risk due to the foods that are the main acrylamide contributors in those populations. In the NLCS study population, Dutch spiced cake was the food that explained most of the variation in acrylamide intake. Dutch spiced cake acrylamide concentrations in 2002 ranged from 260 to 1410 ppb (under the heading of ginger bread in the reference), whereas in French fries the concentration ranged from $<60$ to $1220 \mathrm{ppb}$, and in crisps from 310 to $2800 \mathrm{ppb}$ (18). In other countries, similar concentrations and ranges were reported for French fries and crisps. Apart from some foods having smaller ranges in acrylamide concentrations (which reduces the measurement error when assigning a single value (mean, median) to these foods), the intake of some foods may be easier to report than that of other foods, for instance because they are eaten on a more regular basis. Dutch spiced cake has a smaller range of acrylamide concentrations and may be easier to report on by the study participants.

Lastly, as discussed in the section on multiple comparisons in this Chapter, we were able to investigate the association between acrylamide intake and the risk of several types of cancer, which makes it possible to hypothesise on the possible mode of action based on the pattern of cancer types that were associated with acrylamide intake.

\section{Conclusions and implications}

In this thesis, positive associations between acrylamide intake and endometrial and ovarian cancer risk have been described. These findings have been corroborated by analyses in the Nurses' Health Study (16). The consistency of these findings adds to the evidence that these associations may be causal. Therefore, we conclude that at current dietary intake levels acrylamide may constitute a relevant human endometrial and ovarian cancer risk.

For renal cell cancer and lung cancer in women, the level of confidence in the causality is lower, because these associations were observed only once so far. 
The level of confidence is still lower for oral cavity cancer and oro- and hypopharyngeal cancer, and for the cancer sites for which we observed statistically non-significant positive or inverse associations (prostate, bladder, and progesterone positive and estrogen and progesterone receptor-positive breast cancer).

When future epidemiological acrylamide research corroborates that acrylamide might offer protection against some cancer types, this would then require a calculation of the overall cancer risk, but perhaps more importantly of the net disability and quality-adjusted life years lost, conferred by dietary acrylamide intake.

In the mean time however, based on the precautionary principle, we think that the current approach to strive for acrylamide concentrations in foods that are as low as reasonably achievable (ALARA) should be maintained, and that public health advice should be given by food safety authorities to limit intake of foods containing high concentrations of acrylamide, especially the foods that do not have any known health benefits, such as French fries and potato crisps.

\section{Recommendations for future epidemiological research}

The fact that there are now indications that dietary acrylamide might constitute a higher human cancer risk than anticipated from animal data, should in our opinion lead to more emphasis on epidemiological research into the acrylamide-cancer link and on more use of the epidemiological data in risk assessment, rather than on experimental research on animals.

First and foremost, more prospective studies on the associations that have now been observed are needed, in order to increase confidence that alternative phenomena (chance, confounding) are not responsible for the associations that were observed so far. It is particularly important to know whether acrylamide may also confer a protective action against some types of cancer, as shown by our findings on female lung cancer (and to a lesser extent male oro- and hypopharyngeal and prostate cancer and female bladder cancer). More knowledge on this will eventually make a comprehensive cancer risk assessment for dietary acrylamide possible. Furthermore, there are still relatively common types of cancer that have not yet been studied in relation to dietary acrylamide intake, such as skin cancer and lymphatic and hematological malignancies.

Within future epidemiological studies, stratification based on sex and smoking status is important. Sex may be an effect modifier of the relationship between acrylamide intake and cancer risk, whereas smoking can be both an important confounder of the relationship, as discussed before, and an effect modifier. We further recommend studying effect modification by genetic polymorphisms in acrylamide-metabolising genes, in order to detect possibly more susceptible groups for the carcinogenic effects of acrylamide and to get more insight into the predominant mechanism of action of acrylamide. It would be particularly important to study the role of CYP2E1 (generating glycidamide from acrylamide) and epoxide hydrolase polymorphisms (converting glycidamide into non-reactive glyceramide), because if a hormonal mechanism is the predominant mechanism by which 
acrylamide causes cancer (as suggested in Chapter 9), it is unknown whether the parent compound acrylamide or the metabolite glycidamide is most important. Analyses of these polymorphisms might give some insight into this matter.

With regard to a possible hormonal mechanism of acrylamide carcinogenesis, effect modification by exogenous hormones (contraceptives use, postmenopausal hormone treatment) and reproductive factors (parity, age at menarche and menopause, etc.) would be of interest as well.

Naturally, the studies proposed above have to assess the acrylamide intake well enough to be able to observe true associations. There are some ways by which an indication of the validity of the intake assessment can be obtained. Chapter 2 of this thesis contained the results of a study on whether using mean acrylamide concentrations per food can lead to a reasonable rank ordering of the acrylamide intake of participants of a duplicate diet study. This can be replicated by others for their study population. Furthermore, if possible, we advise to correlate FFQ acrylamide information and the sum of acrylamide and glycidamide haemoglobin adducts in a non-smoking subsample of the study population. If a modest correlation results, this gives confidence in the quality of the FFQ for acrylamide intake assessment.

Finally, we recommend intervention studies with healthy human volunteers to investigate whether dietary acrylamide exposure (via potato crisps, for instance) exerts effects on hormonal systems. 


\section{References}

1. Schottenfeld D, Fraumeni JF, Jr. Cancer Epidemiology and Prevention. New York City: Oxford University Press, 2006.

2. HEATOX. Heat-generated food toxicants: identification, characterisation and risk minimisation, 2007.

3. Goldbohm RA, van den Brandt PA, Dorant E. Estimation of the coverage of Dutch municipalities by cancer registries and PALGA based on hospital discharge data. Tijdschr Soc Gezondheidsz 1994;72:80-84.

4. Goldbohm RA, van den Brandt PA, Brants HA, et al. Validation of a dietary questionnaire used in a largescale prospective cohort study on diet and cancer. Eur J Clin Nutr 1994;48:253-65.

5. Goldbohm RA, van 't Veer P, van den Brandt PA, et al. Reproducibility of a food frequency questionnaire and stability of dietary habits determined from five annually repeated measurements. Eur J Clin Nutr 1995;49:420-9.

6. Bjellaas $\mathrm{T}$, Olesen PT, Frandsen $\mathrm{H}$, et al. Comparison of estimated dietary intake of acrylamide with hemoglobin adducts of acrylamide and glycidamide. Toxicol Sci 2007;98:110-7.

7. Kutting B, Schettgen T, Beckmann MW, Angerer J, Drexler H. Influence of Diet on Exposure to Acrylamide Reflections on the Validity of a Questionnaire. Ann Nutr Metab 2005;49:173-177.

8. Kutting B, Uter W, Drexler $\mathrm{H}$. The association between self-reported acrylamide intake and hemoglobin adducts as biomarkers of exposure. Cancer Causes Control 2008;19:273-81.

9. Wilson KM, Balter K, Adami HO, et al. Acrylamide exposure measured by food frequency questionnaire and hemoglobin adduct levels and prostate cancer risk in the Cancer of the Prostate in Sweden Study. Int J Cancer 2009;124:2384-90.

10. Wilson KM, Vesper HW, Tocco P, et al. Validation of a food frequency questionnaire measurement of dietary acrylamide intake using hemoglobin adducts of acrylamide and glycidamide. Cancer Causes Control 2009;20:269-78.

11. Wirfalt E, Paulsson B, Tornqvist M, Axmon A, Hagmar L. Associations between estimated acrylamide intakes, and hemoglobin AA adducts in a sample from the Malmo Diet and Cancer cohort. Eur J Clin Nutr 2008;62:314-23.

12. Foo LH, Khor GL, Tee ES, Dhanaraj P. Determinants of iron status in Malaysian adolescents from a rural community. Int J Food Sci Nutr 2004;55:517-25.

13. Milman N, Pedersen AN. Blood haemoglobin concentrations are higher in smokers and heavy alcohol consumers than in non-smokers and abstainers-should we adjust the reference range? Ann Hematol 2008.

14. Ortega RM, Lopez-Sobaler AM, Requejo AM, et al. The influence of meat consumption on dietary data, iron status and serum lipid parameters in young women. Int J Vitam Nutr Res 1998;68:255-62.

15. van den Brandt PA, Goldbohm RA, van 't Veer P, Volovics A, Hermus RJ, Sturmans F. A large-scale prospective cohort study on diet and cancer in The Netherlands. J Clin Epidemiol 1990;43:285-95.

16. Summary Report EFSA scientific colloquium no. 11. Acrylamide carcinogenicity new evidence in relation to dietary exposure. 22-23 May 2008, Tabiano (PR), Italy.

17. Hogervorst JG, Schouten LJ, Konings EJ, Goldbohm RA, van den Brandt PA. A Prospective Study of Dietary Acrylamide Intake and the Risk of Endometrial, Ovarian, and Breast Cancer. Cancer Epidemiol Biomarkers Prev 2007;16:2304-2313.

18. Konings EJ, Baars AJ, van Klaveren JD, et al. Acrylamide exposure from foods of the Dutch population and an assessment of the consequent risks. Food Chem Toxicol 2003;41:1569-79. 



\section{Summary}

In 2002, acrylamide, a known rodent carcinogen, was detected in common foods, such as coffee, French fries, potato crisps and cookies (Chapter 1). Due to its ubiquitous presence in foods and concentrations of up to $3 \mathrm{mg} / \mathrm{kg}$ in foods, acrylamide might be responsible for a considerable part of the diet-related cancer cases, according to some researchers involved in acrylamide research. However, their estimates were based on extrapolation of high dose exposure in rats to low dose exposure in humans, and are therefore surrounded by considerable uncertainty. Epidemiological studies can reduce this uncertainty.

The topic of this thesis is the human cancer risk associated with dietary intake of acrylamide. It describes the results of epidemiological analyses of the association between dietary acrylamide intake and the risk of several types of cancer in a prospective cohort study. Because of the wide distribution of acrylamide in the body upon exposure, the association between dietary acrylamide intake and the risk of cancer in many organs was studied.

The analyses took place within the Netherlands Cohort Study on diet and cancer (NLCS), which started in September 1986 with the enrolment of 58,279 men and 62,573 women aged 55-69 years. At baseline, data on dietary habits and other risk factors for cancer were collected by means of a self-administered questionnaire. Data processing and analysis were based on the case-cohort approach, because the desired level of detail of the questionnaire (e.g., open-ended questions) made the processing of the questionnaires of the total cohort infeasible. Therefore, questionnaires were not processed for the total cohort, but only for a subcohort and the cancer cases. Following the case-cohort approach, cancer cases were enumerated for the entire cohort (providing the numerator information for estimating incidence rates), whereas the accumulated person-years at risk for the entire cohort were estimated from a subcohort of 5000 men and women randomly sampled from the entire cohort at baseline (providing the denominator information for estimating incidence rates). Since the start of the study, the subcohort has been followed up regularly for vital status and emigration information. Incident cases in the total cohort have been detected by annual computerised record linkages to the regional cancer registries and the Netherlands Pathology Registry (PALGA). The completeness of cancer followup through linkage with the national cancer registry was estimated to be at least $96 \%$. The follow-up of the subcohort for vital status was nearly $100 \%$ complete at the end of the follow-up period. To study the relationship between acrylamide intake and cancer risks, hazard ratios (type of incidence rate) were calculated using Cox proportional hazards analysis. Because cigarette smoke is a very important source of acrylamide, subgroup analyses were done for non-, and where possible, never-smokers.

In the NLCS, a food frequency questionnaire with questions on 150 foods administered at baseline was used to estimate dietary habits. The acrylamide intake was estimated from the mean acrylamide level of foods from the Dutch market, and the frequency of consumption and portion size of the foods. Using this questionnaire, the mean acrylamide intake in the subcohort was estimated at $22.5 \mu \mathrm{g}$ per day for men and 21.0 for women, which corresponds to 0.29 and $0.32 \mu \mathrm{g} / \mathrm{kg}$ bodyweight per day. 
Acrylamide concentrations vary considerably not only between foods, but also within foods, due to varying processing conditions and the presence of acrylamide precursors. This means that assigning a single acrylamide value to an individual food entails considerable potential for misclassification, and thus the misclassification of acrylamide intake overall may be large. In Chapter 2, we describe the results of a study in which we investigated whether assigning a single mean acrylamide value (the same values as were used in subsequent analyses in the NLCS study) to individual foods results in a sound estimate of the acrylamide intake through a 24-hour duplicate diet. In order to do so, we both estimated and measured the acrylamide content of 3924 -hour duplicate diets. The Spearman correlation coefficient between chemically determined acrylamide content and the estimated acrylamide content of the duplicate diets was $0.82(p<0.001)$. This shows that assigning a single mean acrylamide value to individual foods, which was done to estimate the acrylamide content of the duplicate diets, does not preclude a good rank estimate of the true acrylamide content. The observed correlation strengthens the confidence in the validity of the acrylamide intake assessment in the NLCS.

Chapter 3 presents the results of the analysis of the association between dietary acrylamide intake and postmenopausal endometrial, ovarian and breast cancer risk. We observed increased risks of endometrial and ovarian cancer with increasing dietary acrylamide intake, particularly among never-smokers. The risk of breast cancer was not associated with acrylamide intake. Compared with the lowest quintile of acrylamide intake, multivariable-adjusted hazard ratios (HRs) for endometrial, ovarian, and breast cancer in the highest quintile were 1.29 (95\% confidence interval (Cl): 0.81-2.07), 1.78 (95\% Cl: 1.10-2.88), and 0.93 (95\% Cl: 0.73-1.19), respectively. For never-smokers, the corresponding HRs were 1.99 (95\% Cl: 1.12-3.52), 2.22 (95\% Cl: 1.20-4.08), and 1.10 (95\% $\mathrm{Cl}$ : 0.80-1.52).

The results of the analyses on renal cell, bladder and prostate cancer are described in Chapter 4. We found some indications for a positive association between dietary acrylamide intake and renal cell cancer risk, particularly among long-term smokers. Overall, there were no positive associations with bladder and prostate cancer risk. Compared with the lowest quintile of acrylamide intake, multivariable-adjusted hazard ratios for renal cell, bladder, and prostate cancer in the highest quintile were 1.59 (95\% Cl: 1.09-2.30), 0.91 (95\% Cl: 0.73-1.15), and 1.06 (95\% Cl: 0.87-1.30), respectively. There was an inverse, statistically non-significant, trend for advanced prostate cancer risk in never-smokers. For renal cell cancer, results were the same for men and women, whereas there were some indications for an inverse association with bladder cancer risk in women only.

Chapter 5 describes the associations between dietary acrylamide intake and the risk of gastrointestinal cancers. The multivariable-adjusted HR for the highest quintile of acrylamide intake compared with the lowest was 1.00 (95\% Cl: 0.84-1.20), 1.06 (95\% Cl: 0.781.45), 0.98 (95\% Cl: 0.68-1.40), and 0.83 (95\% Cl: 0.54-1.30) for colorectal, gastric, pancreatic, and oesophageal cancer, respectively. For never or former smokers, there was no 
statistically significant association with acrylamide intake either. There were some significantly increased risks within subgroups defined by body mass index, non-occupational physical activity and age, factors that were a priori selected based on their capacity to modify cytochrome P4502E1 activity. Overall, acrylamide intake was not associated with gastrointestinal cancer risk, but some subgroups deserve attention in future research.

The association between acrylamide intake and lung cancer risk is described in Chapter 6 . The multivariable-adjusted $\mathrm{HR}$ in the highest versus the lowest quintile of intake was 1.03 (95\% Cl: 0.77-1.39) for men, and 0.45 (95\% Cl: 0.27-0.76) for women. The results were not substantially different in never-smokers. Upon investigation of histological subtypes, the inverse association in women was strongest for the histological lung cancer subtype adenocarcinoma.

In Chapter 7, the results of the analyses on brain cancer are described. Compared with the lowest quintile of acrylamide intake, the multivariable-adjusted $\mathrm{HR}$ was 0.87 ( $95 \% \mathrm{Cl}$ : 0.54 1.41 ) in the highest quintile of acrylamide intake. Also in the subgroups based on histology (microscopic verification, astrocytic gliomas, and high-grade astrocytic gliomas) and smoking there was no association

Chapter 8 presents the results for head/neck and thyroid cancer. Dietary acrylamide intake was not associated with an increased risk of oral cavity cancer (multivariableadjusted HR of highest category versus lowest category of acrylamide intake, $95 \% \mathrm{Cl}: 0.72$, $0.36-1.42)$, oro-hypopharynx cancer $(0.61,95 \% \mathrm{Cl}$ : $0.33-1.12)$, larynx cancer $(0.93,95 \% \mathrm{Cl}$ : $0.54-1.58)$ and thyroid cancer $(1.33,95 \% \mathrm{Cl}: 0.70-2.53)$. In non-smoking participants, HRs were not increased either. Stratified by sex, there were indications for a negative association with oral cavity and oro- and hypopharyngeal cancer risk in men. Acrylamide intake was statistically significantly associated with an increased risk of oral cavity cancer in nonsmoking women, but case numbers were small, and thus this result has to be interpreted cautiously.

Both the experimental animal studies on acrylamide carcinogenicity and the epidemiological studies (including our own) on the association between dietary acrylamide intake and cancer risk are summarised and discussed in Chapter 9. Studies with mice so far have involved only subchronic acrylamide exposure (rendering increased incidences of lung and skin tumours) and therefore the current risk assessment of acrylamide is focused on the chronic exposure rat studies. Upon acrylamide administration in drinking water, rats developed tumours of the mammary glands, testes, and thyroid in both of the two studies that were done.

There are epidemiological indications that dietary acrylamide might cause cancer in humans, and the indications are strongest for postmenopausal endometrial and ovarian cancer, for which two prospective cohort studies (including our own) observed statistically significant positive associations. Two prospective cohort studies (among which was our own) have observed some indications for a positive association with postmenopausal 
receptor-positive breast cancer risk, but one more strongly in smokers and the other only in never-smokers and not statistically significant. Further, a positive association was observed with renal cell cancer risk in our own study, and a positive association with oral cavity cancer risk in non-smoking women (although based on few cases) in the same cohort study. More epidemiological studies are needed to investigate the observed relationships in order to be more confident about the causality.

Interestingly, there are also indications from our own study for inverse associations with lung cancer risk in women (statistically significant), advanced prostate cancer risk in never-smoking men, bladder cancer risk in women, and oral cavity and oro- and hypopharyngeal cancer risk in men. However, also these associations need to be investigated in other studies to be able to be more confident about which are true associations and which may have been chance findings.

In the mean time, based on the precautionary principle, we are of the opinion that the current risk management approach to strive for acrylamide concentrations in foods that are as low as reasonably achievable (ALARA) should be maintained. Additionally, public health advice should be given by food safety authorities to limit intake of foods containing high concentrations of acrylamide, especially foods that do not have any known health benefits, such as French fries and potato crisps.

The known risk factors for endometrial and ovarian cancer are primarily of a hormonal nature, which raises the suggestion that acrylamide might have hormonal effects. The observed positive association between acrylamide intake and estrogen and progesterone receptor-positive breast cancer and renal cell cancer risk, and the inverse associations with lung and bladder cancer risk in women and prostate cancer risk might also fit in this hypothesis. The observed pattern of increased and decreased risks can potentially be explained by the presence or predominance of the estrogen receptor isoforms alpha and beta in the tissues that are exposed to acrylamide. The inverse associations, if true, suggest that acrylamide is involved in carcinogenesis in more ways than genotoxicity alone.

The estimated risks, based on the epidemiological studies for the cancer sites for which a positive association was observed, were considerably higher than those based on extrapolations from the rat studies, suggesting that rats may not be a good model for acrylamide-induced human carcinogenesis.

In Chapter 10, the main findings are summarised and discussed within the light of methodological considerations, such as multiple comparisons, confounding, and misclassification of dietary acrylamide exposure. Furthermore, recommendations for future research are given. 


\section{Samenvatting}

In 2002 werd ontdekt dat acrylamide, een stof waarvan bekend is dat die kanker veroorzaakt bij knaagdieren, aanwezig is in alledaagse voedingsmiddelen, zoals koffie, frites, chips en koekjes (Hoofdstuk 1). Gezien de wijdverspreide aanwezigheid en hoge gehalten (tot $3 \mathrm{mg} / \mathrm{kg}$ ) in voeding, zou acrylamide verantwoordelijk kunnen zijn voor een behoorlijk aandeel van de voedingsgerelateerde kankerincidentie, volgens sommige onderzoekers die betrokken zijn bij acrylamide-onderzoek. Hun schattingen zijn echter gebaseerd op extrapolatie van hoge doses in studies met ratten naar lage doses bij mensen en daarom omgeven met een aanzienlijke hoeveelheid onzekerheid. Epidemiologische studies kunnen bijdrage aan een afname van deze onzekerheid.

Het onderwerp van dit proefschrift is de associatie tussen acrylamide-inname via voeding en kanker bij mensen. In het proefschrift worden de resultaten van epidemiologische analyses naar de relatie tussen acrylamide-inname via voeding en het risico op kanker in verschillende organen beschreven.

Deze analyses vonden plaats in het kader van de Nederlandse Cohortstudie naar voeding en kanker (NLCS) die begon in september 1986 met de inclusie van 58279 mannen en 62573 vrouwen van 55 tot en met 69 jaar oud. Bij de start van de studie werden door middel van een door de deelnemers zelf afgenomen vragenlijst gegevens over voeding en andere risicofactoren voor kanker verzameld.

De dataverwerking en -analyse vonden plaats volgens de zogenaamde casecohortbenadering, omdat het gewenste detailniveau van de vragenlijst (bijvoorbeeld de aanwezigheid van open vragen) het onhaalbaar maakte om de vragenlijsten van het hele cohort te verwerken. Om die reden zijn alleen de vragenlijsten van een subcohort en de kankercases verwerkt. Zoals dat gaat in een case-cohort opzet, werden de incidente cases uit het hele cohort opgespoord (leidend tot de teller van de geschatte incidentieratio's), terwijl de opgebouwde persoonstijd "at risk" van het hele cohort werd geschat aan de hand van een subcohort van 5000 , aan het begin van de studie willekeurig uit het hele cohort gekozen, mannen en vrouwen (leidend tot de noemer van de geschatte incidentieratio's). Vanaf het begin van de studie werd het subcohort regelmatig opgevolgd voor informatie over de vitale status en emigratie. Incidente cases werden opgespoord door middel van jaarlijkse elektronische koppeling aan de regionale kankerregistraties en het Pathologisch Anatomisch Landelijk Geautomatiseerd Archief (PALGA). De compleetheid van deze opsporing werd geschat op tenminste $96 \%$. De follow-up van het subcohort was bijna $100 \%$ compleet aan het eind van de follow-upperiode.

Om de relatie tussen acrylamide-inname en kankerrisico te bestuderen berekenden we hazard ratio's (een vorm van incidentieratio) met behulp van Cox proportional hazardsanalyse. Hierbij deden we aparte analyses voor niet-rokers, en waar mogelijk voor nooit-rokers, omdat sigarettenrook een belangrijke bron van acrylamideblootstelling is.

In de NLCS werd aan het begin van de studie een voedselfrequentievragenlijst met vragen over 150 voedingsmiddelen afgenomen om de voedingsgewoonten van de deelnemers te schatten. De acrylamide-inname werd geschat aan de hand van het gemiddelde acrylamidegehalte van verschillende voedingsmiddelen op de Nederlandse markt en de uit 
de vragenlijst verkregen informatie over frequentie en portiegrootte van consumptie van die voedingsmiddelen. De gemiddelde aldus geschatte acrylamide-inname in het subcohort was $22,5 \mu \mathrm{g}$ per dag voor mannen en $21,0 \mu \mathrm{g}$ per dag voor vrouwen, wat overeen komt met 0,29 en $0,32 \mu \mathrm{g} / \mathrm{kg}$ lichaamsgewicht per dag.

Acrylamidegehaltes variëren aanzienlijk tussen, maar ook binnen voedingsmiddelen, door verschillen in productiemethodes en de aanwezigheid van acrylamideprecursors. Dit betekent dat het toekennen van één enkele gemiddelde acrylamidewaarde aan een voedingsmiddel een aanzienlijk gevaar voor misclassificatie met zich mee brengt. Daaruit volgt dat de misclassificatie van de complete acrylamide-inname groot kan zijn. In Hoofdstuk 2 beschrijven we de resultaten van een studie waarin we onderzochten of het toekennen van één enkele gemiddelde acrylamidewaarde aan verschillende voedingsmiddelen (dezelfde gemiddelden als die ook in de latere NLCS-analyses zijn gebruikt) een afdoende schatting van de acrylamide-inname via een compleet dieet gedurende een etmaal oplevert. Hiervoor schatten en maten we de acrylamidehoeveelheid in 39 24-uurs duplicaatvoedingen. De Spearman correlatiecoëfficiënt voor de correlatie tussen de chemisch geanalyseerde en geschatte acrylamidehoeveelheid van de duplicaatvoedingen was $0,82(p<0,001)$. Dit geeft aan dat het toekennen van één enkele acrylamidewaarde aan een voedingsmiddel, op basis waarvan de acrylamidehoeveelheid in de duplicaatvoedingen was geschat, een goede orde-van-grootte schatting van de totale acrylamidehoeveelheid mogelijk maakt. De geobserveerde correlatie geeft vertrouwen in de validiteit van de acrylamide-innameschatting in de NLCS.

In Hoofdstuk 3 worden de resultaten van analyses naar het verband tussen acrylamideinname via de voeding en het risico op postmenopausale baarmoeder-, eierstok- en borstkanker beschreven. We zagen verhoogde risico's op baarmoeder- en eierstokkanker bij toenemende acrylamide-inname, vooral bij nooit-rokende vrouwen. Er was geen verband met borstkanker. De voor verstorende variabelen gecorrigeerde hazard ratio (HR) in het hoogste ten opzichte van het laagste kwintiel van inname was 1,29 (95\% betrouwbaarheidsinterval (bi): 0,81-2,07) voor baarmoeder-, 1,78 (95\% bi: 1,10-2,88) voor eierstok- en 0,93 (95\% bi: 0,73-1,19) voor borstkanker. Voor nooit-rokende vrouwen waren de overeenkomstige HR's 1,99 (95\% bi: 1,12-3,52), 2,22 (95\% bi: 1,20-4,08) en 1,10 (95\% bi: $0,80-1,52)$.

De resultaten van de analyses voor nier-, blaas- en prostaatkanker zijn beschreven in Hoofdstuk 4. We vonden aanwijzingen voor een positief verband tussen de acrylamideinname en het risico op nierkanker, vooral bij langdurige rokers. Er was geen positief verband met het risico op blaas- en prostaatkanker. De voor verstorende variabelen gecorrigeerde $\mathrm{HR}$ in het hoogste ten opzichte van het laagste kwintiel van inname was 1,59 (95\% bi: 1,09-2,30) voor nier-, 0,91 (95\% bi: 0,73-3,52) voor blaaskanker en 1,06 (95\% bi: $0,87-1,30)$ voor prostaatkanker. Er was een statistisch niet-significante inverse relatie voor gevorderde prostaatkanker bij nooit-rokers. Voor nierkanker waren de resultaten 
voor mannen en vrouwen gelijk, terwijl er alleen voor vrouwen aanwijzingen waren voor een invers verband met het blaaskankerrisico.

In Hoofdstuk $\mathbf{5}$ staan de resultaten van de relatie tussen acrylamide-inname en het risico op kanker van het gastrointestinale systeem. De voor verstorende variabelen gecorrigeerde HR in het hoogste ten opzichte van het laagste kwintiel van inname was 1,00 (95\% bi: 0,84-1,20) voor colorectaalkanker, 1,06 (95\% bi: 0,78-1,45) voor maag-, 0,98 (95\% bi: 0,68$1,40$ ) voor alvleesklier- en 0,83 (95\% bi: $0,54-1,30)$ voor slokdarmkanker. Ook voor nooitof ex-rokers waren er geen statistisch significante verbanden. In enkele subgroepen, gebaseerd op Quetelet index, niet-beroepsmatige fysieke activiteit en leeftijd (factoren die mogelijk van invloed zijn op de activiteit van het enzym cytochroom P4502E1), waren er statistisch significant verhoogde acrylamide-geassocieerde risico's. Over het geheel genomen was acrylamide-inname dus niet geassocieerd met het risico op gastrointestinale tumoren, maar bepaalde subgroepen verdienen aandacht in toekomstig onderzoek.

Het verband tussen acrylamide-inname en het risico op longkanker wordt beschreven in Hoofdstuk 6. De voor verstorende variabelen gecorrigeerde HR in het hoogste ten opzichte van het laagste kwintiel van inname was 1,03 (95\% bi: 0,77-1,39) voor mannen en 0,45 $(95 \%$ bi: $0,27-0,76)$ voor vrouwen. De resultaten voor nooit-rokers waren vergelijkbaar. Analyse op basis van het histologische subtype van de tumor liet zien dat de inverse relatie bij vrouwen het sterkst was voor het histologische longkankersubtype adenocarcinoom.

In Hoofdstuk 7 worden de analyses voor hersentumoren beschreven. Vergeleken met de $H R$ in het laagste kwintiel van inname was de voor verstorende variabelen gecorrigeerde HR in het hoogste kwintiel 0,87 (95\% bi: 0,54-1,41). We vonden ook geen verband met acrylamide in subgroepen van histologische subtypes van hersentumoren (microscopisch geverifieerde tumoren, astrocytische gliomen, en hooggradige astrocytische gliomen) of bij nooit-rokers.

De relatie tussen acrylamide-inname en het risico op hoofd/hals- en schildklierkanker, tenslotte, staat beschreven in Hoofdstuk 8. Er was geen verband tussen acrylamideinname en het risico op mondholtekanker (de voor verstorende variabelen gecorrigeerde $\mathrm{HR}$ in het laagste kwintiel van inname was $0,72,95 \%$ bi: $0,36-1,42)$, oro- en hypofarynxkanker $(0,61,95 \%$ bi: 0,33-1,12), larynxkanker (0,93, 95\% bi: 0,54-1,58) en schildklierkan$\operatorname{ker}(1,33,95 \%$ bi: $0,70-2,53)$. Ook voor niet-rokers waren er geen verhoogde HR's. Als we keken naar mannen en vrouwen afzonderlijk, waren er aanwijzingen voor een invers verband met mondholte- en oro- en hypofarynxkanker bij mannen. Acrylamide-inname was statistisch significant geassocieerd met een verhoogd risico op mondholtekanker bij niet-rokende vrouwen, maar het aantal kankergevallen in deze analyse was klein. Daarom moet deze bevinding met voorzichtigheid worden geïnterpreteerd.

In Hoofdstuk 9 worden de experimentele dieronderzoeken en de epidemiologische studies (waaronder de onze) naar acrylamide en kanker samengevat en bediscussieerd. De 
studies die tot dusver zijn uitgevoerd met muizen betroffen slechts subchronische blootstelling (leidend tot een verhoogde incidentie van long- en huidtumoren) en daarom is de huidige kankerrisicoschatting van acrylamide gebaseerd op de chronische blootstellingssstudies met ratten. Deze ontwikkelden tumoren in borstklieren, teelballen en schildklier na acrylamidetoediening via drinkwater in de beide studies die zijn uitgevoerd.

Er zijn epidemiologische aanwijzingen dat acrylamide in de voeding kanker in mensen kan veroorzaken en die aanwijzingen zijn het sterkst voor baarmoeder- en eierstokkanker, waarvoor twee prospectieve cohortstudies (waaronder die van ons) een statistisch significant positief verband vonden. Twee prospectieve studies (waaronder opnieuw die van onszelf) hebben ook een positief verband gevonden voor postmenopausale receptorpositieve borstkanker; de een sterker in rokers en de ander alleen in nooit-rokers en niet statistisch significant. Verder was er een positieve associatie tussen acrylamide en het nierkankerrisico in onze studie en een positief verband met het risico op mondholtekanker bij niet-rokende vrouwen (gebaseerd op een klein aantal kankergevallen) in dezelfde studie. Er zijn meer epidemiologische studies nodig om de waargenomen verbanden te onderzoeken en zo een beter beeld van de causaliteit te verkrijgen.

Aan de andere kant leverde onze studie ook aanwijzingen voor een invers verband met het risico op longkanker bij vrouwen (statistisch significant), gevorderde prostaatkanker bij nooit-rokers, blaaskanker bij vrouwen en oro- en hypofarynxkanker bij mannen. Deze verbanden moeten ook in andere studies onderzocht worden om meer zekerheid te krijgen over welke bevindingen echte verbanden zijn en welke mogelijk kansbevindingen waren.

Wij zijn van mening dat in de tussentijd het voorzorgsprincipe gehanteerd moet worden bij het risicomanagement van acrylamide en dat het uitgangspunt dat de acrylamidegehaltes in voeding zo laag mogelijk moeten zijn ("as low as reasonably achievable"(ALARA)) gehandhaafd moet blijven. Ter aanvulling moet er publieksvoorlichting worden gegeven door voedingsinstanties om de consumptie van voedingsmiddelen met hoge acrylamidegehaltes te beperken, met name van voedingsmiddelen die geen gekende gezondheidsvoordelen opleveren, zoals frites en chips.

De bekende risicofactoren voor baarmoeder- en eierstokkanker zijn voornamelijk van een hormonale aard, waardoor de hypothese opgeworpen kan worden dat acrylamide hormonale effecten zou kunnen hebben. De waargenomen associatie tussen acrylamideinname en oestrogeen- en/of progesteronreceptor-positieve borstkanker en de inverse associaties met long- en blaaskanker bij vrouwen kan ook in deze hypothese passen. Het waargenomen patroon van verhoogde en verlaagde risico's zou verder verklaard kunnen worden door de aanwezigheid of overheersendheid van de oestrogeenreceptorsubtypes alpha of beta in de weefsels waarmee acrylamide in contact komt. De inverse verbanden zijn, als ze waar zijn, niet in overeenstemming met de heersende hypothese dat acrylamide kanker veroorzaakt door alleen maar genotoxische mechanismen.

De acrylamiderisicoschattingen uit de epidemiologische studies die een positief verband met kanker waarnamen zijn aanzienlijk hoger dan de schattingen die gebaseerd zijn op extrapolatie vanuit de studies met ratten, wat erop zou kunnen duiden dat ratten geen goed model zijn voor acrylamide-geïnduceerde kanker bij mensen. 
In Hoofdstuk 10 worden de belangrijkste bevindingen samengevat en bediscussieerd in het licht van methodologische beperkingen, zoals "multiple comparisons", confounding en misclassificatie van de acrylamide-inname via de voeding. Verder worden er aanbevelingen voor toekomstig onderzoek gegeven. 



\section{Dankwoord}

Velen hebben een bijdrage geleverd aan het tot stand komen van dit proefschrift.

Alleerst wil ik mijn promotor en beide co-promotoren bedanken.

Piet, bedankt voor de plezierige begeleiding. Ik heb zelden iemand ontmoet die de dingen zo scherp ziet en formuleert als jij. Ik heb ook veel waardering voor je humor, zoals die keer dat ik vol spanning mijn eerste artikel met je kwam bespreken. "tja, dit is dus helemaal niks hè", gevolgd door een voor mij tergend lange stilte en de woorden "Grapje. Kun jij wel hebben, toch?".

Leo, je bent een uitstekende begeleider; laagdrempelig, geïnteresseerd, betrokken, vol van handige tips en tricks, altijd in voor een gezellig praatje over "onze" hormoontheorie, epidemiologie versus toxicologie en van alles en nog wat. We hebben een interessante weg door de wondere wereld van acrylamide afgelegd en die weg gaat gelukkig nog verder hierna.

Erik, mede dankzij jou is de samenwerking met de Voedsel en Waren Autoriteit in de vorm van dit project tot stand gekomen. Van een afstandje was je altijd heel betrokken en stipt met het aanleveren van je nuttige commentaar op de stukken. Bedankt!

Sandra Bausch, jouw kritische commentaar op mijn manuscripten was vaak een eyeopener en daarom uitermate nuttig. Het was aangenaam samenwerken!

Alle andere mensen die deel uitmaken van het NLCS-team (sommigen zelfs al 21 jaar!), uit Maastricht en van TNO en RIVM: dank voor jullie bijdrage in welke vorm dan ook.

Mijn kamergenoten gedurende het project: Boukje en Jessie. Met jullie heb ik vele leuke gesprekken gehad over wetenschap, maar ook over allerlei andere dingen die het leven leuk (en soms minder leuk) maken. Dank voor jullie plezierige gezelschap!

Brenda, Mirjam, Audrey, Stefan, Bas, John P., Monique M., Christel: jullie maakten mijn epi-leven extra kleurrijk. Brenda, wanneer gaan we weer op congres?

Bas, de ontwerper van de omslag van dit proefschrift: je bent een echte kunstenaar. Nog voordat ik de zelfgemaakte foto's van zelf verbrande papierrandjes (waarbij ik bijna mijn keuken in de hens had gezet) naar je had opgestuurd, had je die zelf al bij elkaar getoverd. Ik ben heel benieuwd naar je masterpiece (drieluik) straks!

Harry en Jos, de ICT-mannen, volgens mij heb ik jullie af en toe voor heel onbekende en overklaarbare problemen gesteld met mijn computerkunsten.

Veel dank gaat uit naar de begeleidingscommissie, die jaarlijks de voortgang van dit project in de gaten hield. Hierbij heb ik van de ervaring en expertise van een aantal deskundigen gebruik kunnen maken. Bert-Jan Baars, Floor van Leeuwen, Evert Schouten, Peter Zweipfenning en Rob Theelen, dank!

Linda, John en Mark van VWA Zuid: hartelijk dank voor jullie hulp bij de duplicaatvoedingsstudie (Linda) en de vele analyses van acrylamide in voedingsmiddelen (John en Mark). 
Veel dank ook aan Hans van Egmond en Eric Sizoo van het Laboratorium voor Analytisch Voedings- en Residu-onderzoek van het RIVM voor het verstrekken van de duplicaatvoedingen en de fijne samenwerking.

Grete, thank you for being my intern and doing a great job on the estrogen and progesterone receptor-defined breast cancer analyses. I wish you all the best in your own PhD project and of course in your private project (baby)!

Marlies, jij bent nu op dit moment nog even mijn stagiaire en hebt geen directe bijdrage geleverd aan dit boekje, maar de leuke discussies met jou over acrylamide en het risico op lymfatische maligniteiten maken dat mijn enthousiasme over acrylamide er alleen maar groter op wordt. We gaan er een mooi artikel van maken!

Vrienden zorgden de afgelopen jaren voor (ont)spanning, zodat het werk goed vol te houden was.

Debby, Kasper en Mirjam, Gilbert en Angelina, Ronald en Denise, Roel, Nathan en Manon, Boukje, en Daksha, dank jullie wel! Ik hoop dat ik een aantal van jullie de komende tijd weer eens wat vaker zal gaan zien.

En oud-MGK-medestudenten: ik mis de MGK-weekendjes die we vroeger regelmatig hadden!

Maud en Tim, mijn paranimfen. Het voelt goed om te weten dat er straks twee rotten in het vak achter me staan en dat het cirkeltje nu rond is. Maud, in het Leuvense zaten wij bij Tim's promotie vanuit de zaal te supporteren, en Tim, bij Maud hebben wij tweeën ontzettend goed geparanimfd, al zeiden we het zelf.

Maud, jij begrijpt mij altijd bijna zonder dat ik iets hoef te zeggen (dat laatste is trouwens ook niet altijd eenvoudig bij jou, haha).

Tim, jij bent een heel bijzondere levensgenieter (als je je niet teveel zorgen maakt over alle milieugezondheidkundige problemen); het is goed toeven in jouw omgeving, met Ellen, je prachtige kinderen en je beestjes!

Lieve Gerard, Netty en Sil, dank voor jullie liefde, steun en interesse in mijn werk.

Gerard, door onze epi-discussies was ik erg op mijn hoede om niet "effect" te schrijven waar ik "association" bedoel. Ik heb zojuist nog de hopelijk laatste "search" en "replace" gedaan.

Lieve Joost, Sanne, Renee, pap en mam, we zijn een bijzonder gezinnetje. Ik dank jullie uit het diepst van mijn hart voor de liefde en steun die ik kreeg, in het bijzonder ook de afgelopen tijd.

Oh ja, pap en mam, ik ben inmiddels zover dat ik trots ben op de bijzondere "doop"namen die jullie me gaven en die nu zo prachtig prijken op pagina 3 van dit proefschrift! 
Marco. Hoe dierbaarder de mensen worden in deze lijst, hoe moeilijker het me valt om ze toe te spreken op papier. En zo hoort het ook, denk ik. Bij jou aangekomen schieten woorden tekort. Ik zeg dus maar niks meer, juist omdat jij alles voor me bent.

Eén ding nog: dat je uitgerekend vandaag (de laatste dag van mijn proefschriftbezigheden) een peperkoek hebt gekocht, omdat je daar ineens zo'n onbedwingbare zin in had, zegt wel iets over de waarde die je hecht aan mijn onderzoek......

Ach liefke, doe mij ook maar een plakje (met roomboter); het leven moet vooral ook genieten zijn, samen!

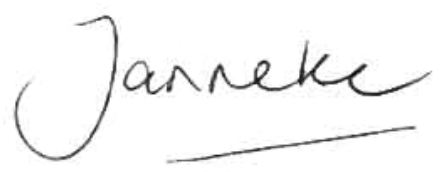

217 



\section{About the author}

Janneke Hogervorst was born on December 81973 in Nijmegen, The Netherlands.

She completed secondary school (Gymnasium) at the Sint Maartenscollege in Maastricht in 1993. After that, she worked in a company casting bronze sculptures for artists until 1996, when she started studying Environmental Health Sciences (Faculty of Health Sciences) at Maastricht University. During this study, she fulfilled an internship at the Centre for Environmental Sciences at the University of Hasselt on house dust as a source of exposure to cadmium and lead in the historically polluted Noorderkempen in Belgium. In addition, she followed extra courses on Epidemiology, resulting in the registration as an Epidemiologist A. She graduated in Environmental Health Sciences (cum laude) in 2002. From 2002 through 2004, she worked as a junior researcher at the Department of General Practice at Maastricht University on the topic of ambient airborne particulate matter pollution and respiratory health of primary school children. From March 2005 until March 2009, she worked on the PhD research described in this thesis at the Department of Epidemiology (GROW - School for Oncology and Developmental Biology -, Maastricht University). From April 2009 onwards, Janneke has been working as a postdoc at the Department of Epidemiology (GROW, Maastricht University) on acrylamide and its association with hormonal systems and its interaction with genes involved in acrylamide metabolism and hormonal systems. 



\section{List of publications}

\section{Submitted manuscripts}

Konings EJM, Hogervorst JGF, van Rooij L, Schouten LJ, Sizoo EA, van Egmond HP, Goldbohm RA, van den Brandt PA. Variability of acrylamide concentrations in single foods does not preclude assessing dietary acrylamide intake through questionnaires: a validation study from the Netherlands.

Hogervorst JGF, Schouten LJ, Konings EJM, Goldbohm RA, van den Brandt PA. The carcinogenicity of dietary acrylamide intake: a side by side discussion of epidemiological and toxicological research.

Pedersen GS, Hogervorst JGF, Schouten LJ, Konings EJM, Goldbohm RA, van den Brandt PA. Dietary acrylamide intake and estrogen and progesterone receptor-defined postmenopausal breast cancer risk.

\section{Peer reviewed publications}

de Kok, T.M., J.G. Hogervorst, J.C. Kleinjans, and J.J. Briede, Radicals in the church. Eur Respir J, 2004. 24(6): p. 1069-70.

Godschalk, R., J. Hogervorst, H. Albering, P. Mercelina-Roumans, F.J. van Schooten, J. de Haan, and J. Kleinjans, Interaction between cadmium and aromatic DNA adducts in hprt mutagenesis during foetal development. Mutagenesis, 2005. 20(3): p. 181-5.

Briede, J.J., T.M. De Kok, J.G. Hogervorst, E.J. Moonen, C.L. Op Den Camp, and J.C. Kleinjans, Development and application of an electron spin resonance spectrometry method for the determination of oxygen free radical formation by particulate matter. Environ Sci Technol, 2005. 39(21): p. 8420-6.

de Kok, T.M., J.G. Hogervorst, J.J. Briede, M.H. van Herwijnen, L.M. Maas, E.J. Moonen, H.A. Driece, and J.C. Kleinjans, Genotoxicity and physicochemical characteristics of trafficrelated ambient particulate matter. Environ Mol Mutagen, 2005. 46(2): p. 71-80.

Hogervorst, J.G., T.M. de Kok, J.J. Briede, G. Wesseling, J.C. Kleinjans, and C.P. van Schayck, Relationship between Radical Generation by Urban Ambient Particulate Matter and Pulmonary Function of School Children. J Toxicol Environ Health A, 2006. 69(3): p. 245-62.

Nawrot, T., M. Plusquin, J. Hogervorst, H.A. Roels, H. Celis, L. Thijs, J. Vangronsveld, E. Van Hecke, and J.A. Staessen, Environmental exposure to cadmium and risk of cancer: a prospective population-based study. Lancet Oncol, 2006. 7(2): p. 119-26. 
de Kok TM, Driece HA, Hogervorst JG, Briede JJ. Toxicological assessment of ambient and traffic-related particulate matter: A review of recent studies. Mutat Res 2006;613(23):103-22.

Hogervorst J, Plusquin M, Vangronsveld J, et al. House dust as possible route of environmental exposure to cadmium and lead in the adult general population. Environ Res 2007;103(1):30-7.

van Breda SGJ, Hogervorst JGF, Schouten LJ, et al. Toenails: an easily accessible source of DNA for genetic analyses in large-scale epidemiological studies. Clinical Chemistry 2007;53(6):1168-1170.

Hogervorst JG, Schouten LJ, Konings EJ, Goldbohm RA, van den Brandt PA. A Prospective Study of Dietary Acrylamide Intake and the Risk of Endometrial, Ovarian, and Breast Cancer. Cancer Epidemiol Biomarkers Prev 2007;16:2304-2313.

Hogervorst JG, Schouten LJ, Konings EJ, Bausch-Goldbohm RA, van den Brandt PA. Dietary acrylamide intake and the risk of renal cell, bladder, and prostate cancer. Am J Clin Nutr 2008;87:1428-38.

Hogervorst JG, Schouten L, Konings EJ, Goldbohm RA, van den Brandt PA. Acrylamide in food and the risk of gastrointestinal cancer: a prospective cohort study. J Nutr 2008;138:2229-36.

Chantal E.H. Dirix, Janneke G. F. Hogervorst, Patrick Rump, Johannes J.E. Hendriks, Maaike Bruins, Gerard Hornstra. Prenatal arachidonic acid exposure and selected immune-related variables in childhood. Br J Nutr 2009; epub ahead of print.

Hogervorst JG, Schouten L, Konings EJ, Goldbohm RA, van den Brandt PA. Lung cancer risk in relation to dietary acrylamide intake. Hogervorst JG, Schouten $L$, Konings EJ, BauschGoldbohm RA, van den Brandt PA. J Nat Cancer Inst 2009;101:651-62.

Hogervorst JG, Schouten L, Konings EJ, Goldbohm RA, van den Brandt PA. Dietary acrylamide intake and brain cancer risk. Cancer Epidemiol Biomarkers Prev 2009;18:1663-6.

Schouten LJ, Hogervorst JGF, Konings EJM, Goldbohm RA, van den Brandt PA. Dietary Acrylamide Intake and the Risk of Head-neck and Thyroid Cancer: Results from the Netherlands Cohort Study. Am J Epidemiol, in press.

Hogervorst JGF, Schouten L, van den Brandt PA. Reaction on Gargas et al.: Acrylamide: Consideration of species differences and nonlinear processes in estimating risk and safety for human ingestion. In press. 


\section{National (refereed) journals}

van Schayck, C.P., J.G.F. Hogervorst, T.M.C.M. De Kok, G. Wesseling, J.J. Briede, and J.C.S. Kleinjans, Relatie tussen de samenstelling van fijn stof in de lucht en de longfunctie van schoolkinderen. Ned Tijdschr Geneesk, 2006. 150(13): p. 735-40.

\section{Books, or contributions to books}

Konings EJ, Hogervorst JGF, Schouten LJ, van den Brandt PA. Assessing exposure levels of acrylamide. In: Skog K, Alexander J, eds. Acrylamide and other hazardous compounds in heat-treated foods. Cambridge: Woodhead Publishing Limited, 2006: 214-225.

\section{Abstracts/invited lectures}

Dietary acrylamide and endometrial, ovarian and breast cancer. Oral presentation at NWO Food Research day 2006, Deurne, The Netherlands.

Dietary acrylamide and endometrial, ovarian and breast cancer. Invited oral presentation at the Department of Human Food at Wageningen University, 2006, Wageningen, The Netherlands.

A prospective study of dietary acrylamide intake and renal cell cancer. Poster presentation at the American Association for Research on Cancer annual meeting 2007, Los Angeles, USA.

Acrylamide in food and the risk of renal cell, bladder and prostate cancer. Oral presentation at WEON 2007, Maastricht, The Netherlands.

A prospective study of dietary acrylamide intake and renal cell cancer. Poster presentation at the Masterclass Diet and Cancer of Wageningen University, 2007, Wageningen, The Netherlands.

A prospective study of dietary acrylamide intake and renal cell cancer. Poster presentation at the GROW Science Day 2007, Maastricht, The Netherlands.

Acrylamide in food and the risk of gastrointestinal cancer. Oral presentation at WEON 2008, Groningen, The Netherlands.

A Prospective Study of Dietary Acrylamide Intake and the risk of endometrial, ovarian, and breast cancer. Invited oral presentation at a meeting of the European Commission CONTAM panel, 2008, Brussels, Belgium.

Lung cancer risk in relation to dietary acrylamide intake. Poster presentation at the annual meeting of the European Environmental Mutagen Society 2008, Cavtat, Croatia. 